\title{
Water-Quality and Lake-Stage Data for Wisconsin Lakes, Water Year 2004
}

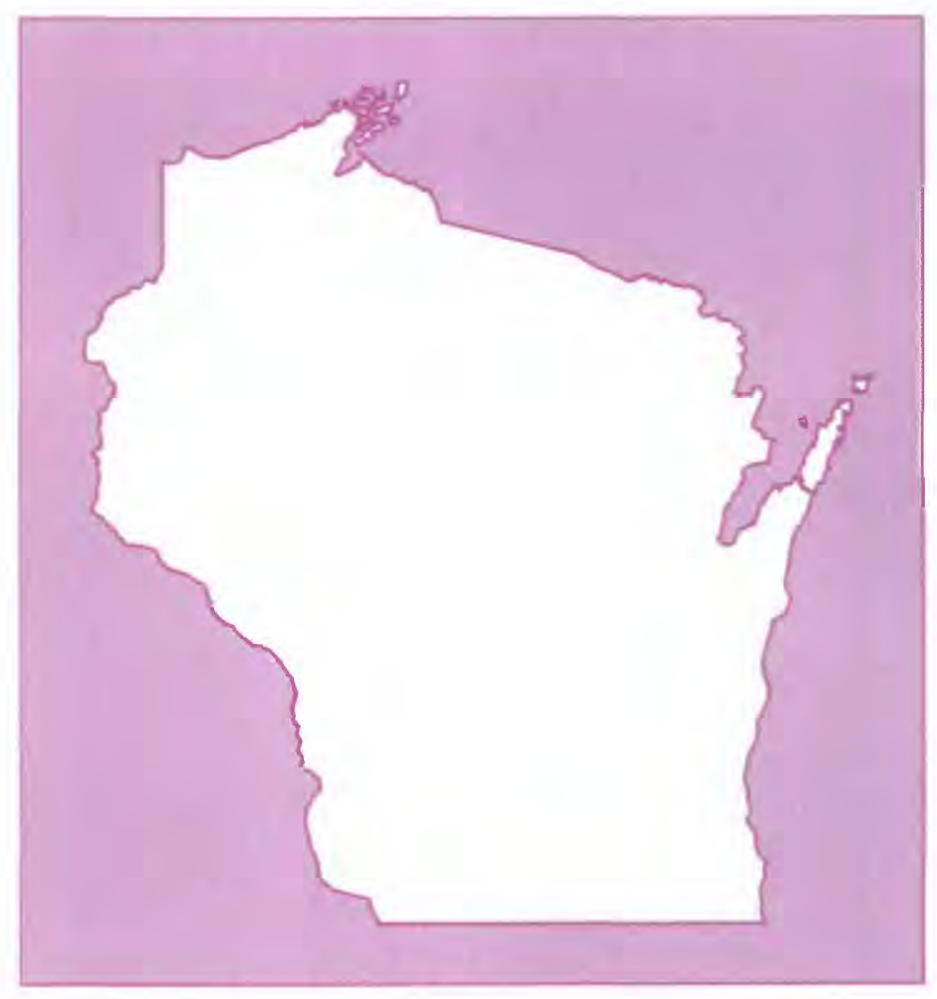

U.S. GEOLOGICAL SURVEY

Open-File Report 2005-II47

Prepared in cooperation with the State of Wisconsin and local agencies 

U.S. Department of the Interior

U.S. Geological Survey

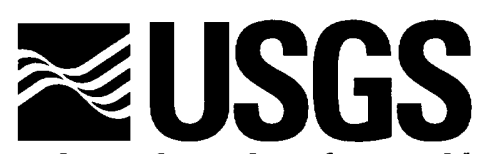

science for a changing world

\section{Water-Quality and Lake-Stage \\ Data for Wisconsin Lakes, \\ Water Year 2004}

A report by the Wisconsin District Lake-Studies Team-

W.J. Rose (team leader), H.S. Garn, G.L. Goddard, S.B. Marsh, D.L. Olson, and D.M. Robertson

Open-File Report 2005-1147

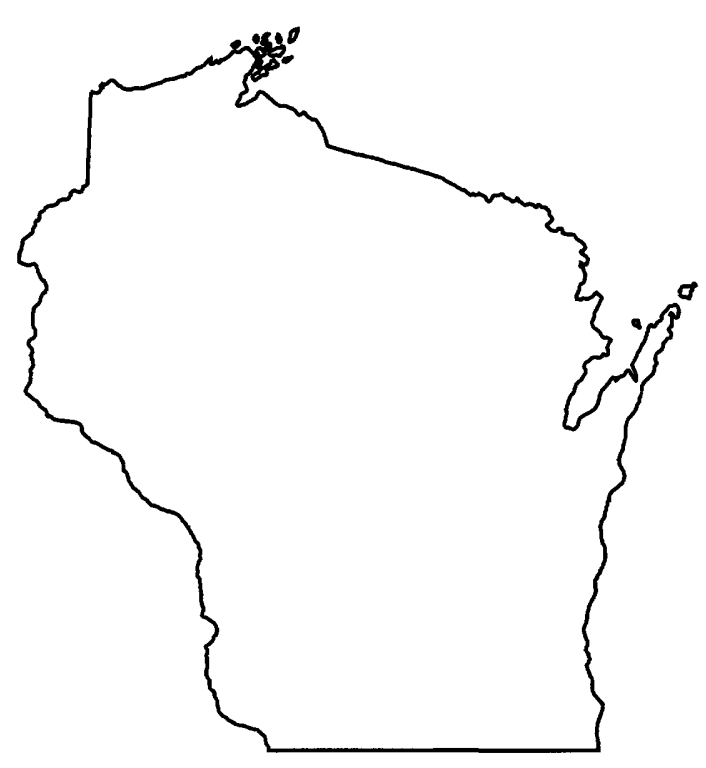

Prepared in cooperation with the

State of Wisconsin and with other agencies 


\title{
U.S. DEPARTMENT OF THE INTERIOR GALE A. NORTON, Secretary
}

\author{
U.S. GEOLOGICAL SURVEY \\ Charles G. Groat, Director
}

For additional information write to:

Copies of this report can be purchased from:

Center Director

U.S. Geological Survey

8505 Research Way

Middleton, Wl 53562
U.S. Geological Survey

Earth Science Information Center

Open-File Reports Section

Box 25286, MS 517

Denver Federal Center

Denver, CO 80225 


\section{CONTENTS}

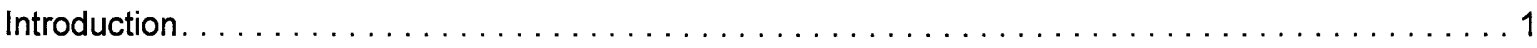

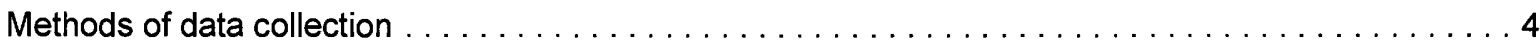

Explanation of physical and chemical characteristics of lakes $\ldots \ldots \ldots \ldots \ldots \ldots \ldots \ldots$

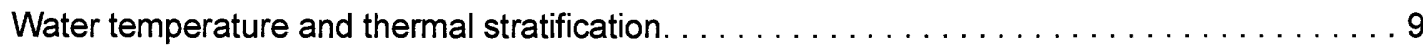

Specific conductance. . . . . . . . . . . . . . . . . . . . . . . . . . . . . . . . . 11

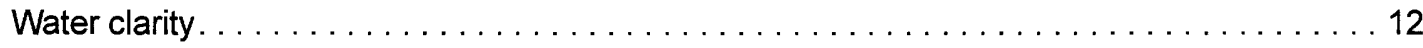

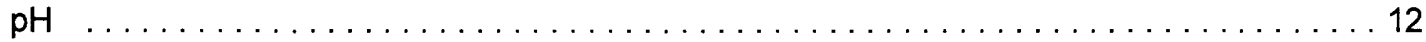

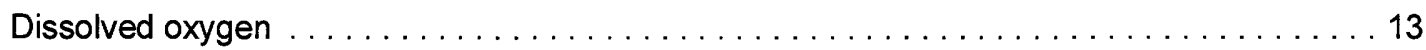

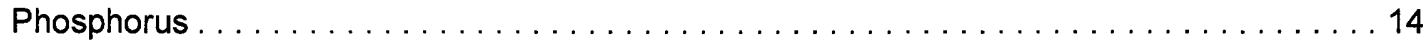

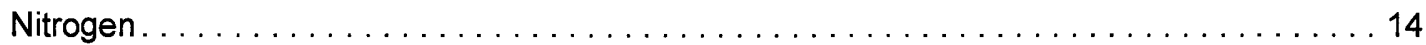

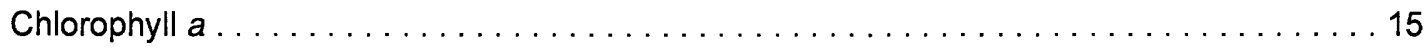

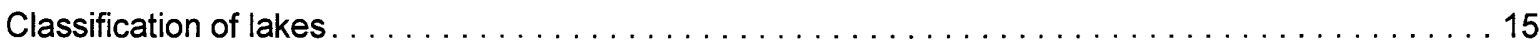

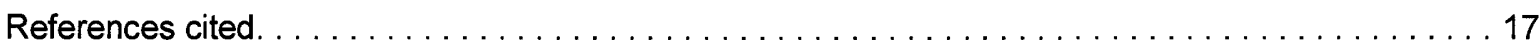

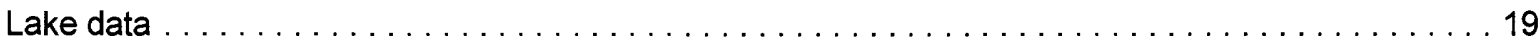

Big Cedar

North Site, near West Bend, $432409088151600 \ldots \ldots \ldots \ldots \ldots \ldots$. . . . . . . . . . . 21

South Site, near West Bend, $432224088154900 \ldots \ldots \ldots \ldots \ldots \ldots \ldots \ldots$

Butternut

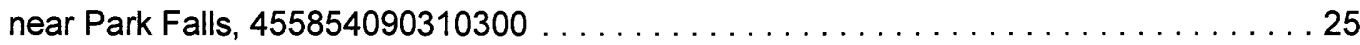

Deep Hole, near Park Falls, $455803090310800 \ldots \ldots \ldots \ldots \ldots \ldots$. . . . . . . . . . 26

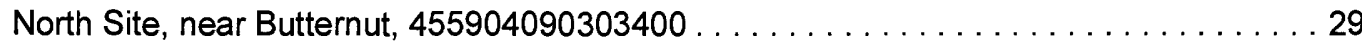

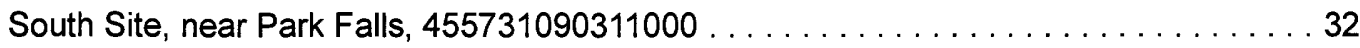

Far South Site, near Park Falls, $455651090312700 \ldots \ldots \ldots \ldots \ldots \ldots \ldots \ldots \ldots$

Delavan

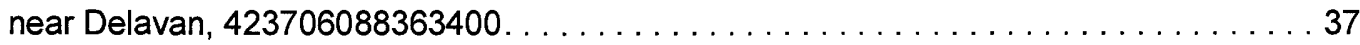

at Center near Delavan Lake, $423556088365001 \ldots \ldots$. . . . . . . . . . . . . . . . . . . 38

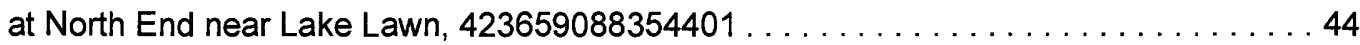

at SW End near Delavan Lake, 423526088380101 . . . . . . . . . . . . . . . . . . . . . . . . 44

Desair . . . . . . . . . . . . . . . . . . . . . . . . . . . . . . . . . . . . . . . . . 45

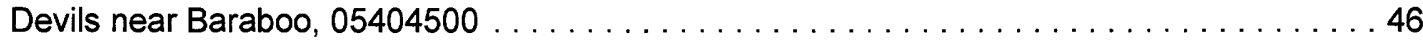

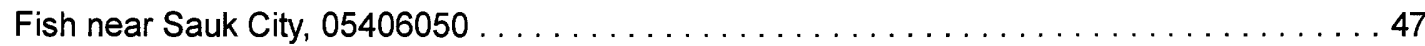

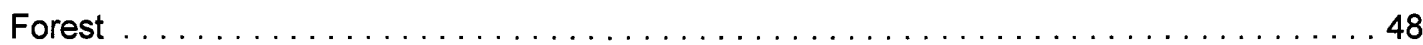

Geneva

at Lake Geneva, $423525088260400 \ldots \ldots \ldots \ldots \ldots$. . . . . . . . . . . . . . . . . . . . . . . 50

West End, near Williams Bay, $423329088323300 \ldots \ldots \ldots \ldots \ldots \ldots \ldots \ldots \ldots$

Green

at County Trunk Highway A near Green Lake (East End), 434928088553601 . . . . . . . . 57

Deep Hole, 434756089020500 . . . . . . . . . . . . . . . . . . . . . . . . . . . . . . 58

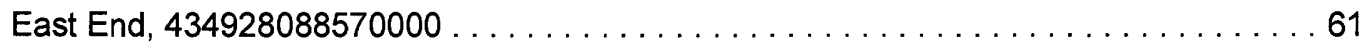

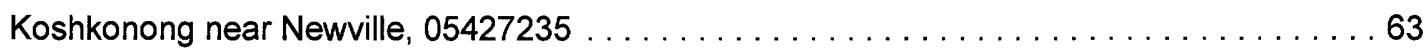




\section{CONTENTS--Continued}

Little Cedar

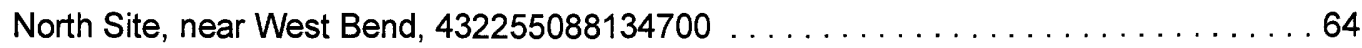

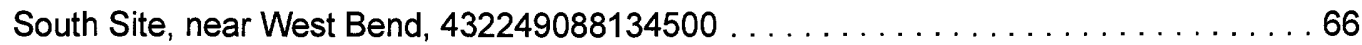

Mendota at Madison, $05428000 \ldots \ldots \ldots \ldots \ldots$. . . . . . . . . . . . . . . . . . . . . . . 68

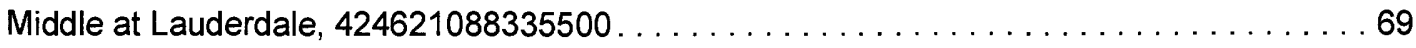

Middle Genesee near Oconomowoc, $430309088284800 \ldots \ldots \ldots \ldots \ldots \ldots \ldots \ldots \ldots$

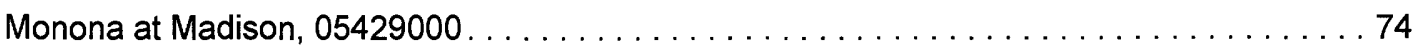

Muskego (Big Muskego) near Wind Lake, $425109088075000 \ldots \ldots \ldots \ldots \ldots \ldots \ldots$

Nagawicka

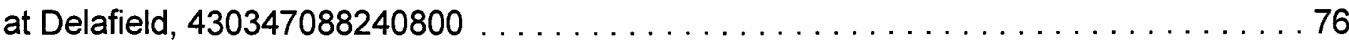

at Deep Hole, at Delafield, $430417088230300 \ldots \ldots \ldots \ldots$. . . . . . . . . . . . . 77

Oconomowoc

No. 1 (Center) at Oconomowoc, $430551088273500 \ldots \ldots \ldots \ldots \ldots \ldots \ldots \ldots$. . . . . . 80

No. 2 (off Hewitt Point) at Oconomowoc, $430609088262200 \ldots \ldots \ldots \ldots \ldots$. . . . . 82

Okauchee

at Okauchee, $430723088252100 \ldots \ldots \ldots \ldots \ldots \ldots \ldots \ldots \ldots$. . . . . . . . . . . 84

No. 1, near Okauchee, $430759088244200 \ldots \ldots \ldots \ldots$. . . . . . . . . . . . . . . 86

No. 2, at Okauchee, $430645088264500 \ldots \ldots \ldots \ldots \ldots \ldots$. . . . . . . . . . . 88

No. 3, at Okauchee, $430642088252400 \ldots \ldots \ldots \ldots \ldots \ldots$. . . . . . . . . . . . . 90

No. 4, at Okauchee, $430757088261700 \ldots \ldots$. . . . . . . . . . . . . . . . . . . . . . . . 92

Potter near Mukwonago, $424905088204000 \ldots \ldots \ldots \ldots \ldots \ldots \ldots . \ldots \ldots$

Powers at Powers Lake, $423246088175800 \ldots \ldots \ldots \ldots$. . . . . . . . . . . . . . . . 97

Spooner

Deep Hole, near Spooner, 455034091493300 . . . . . . . . . . . . . . . . . . . . . 100

Southeast Site, near Spooner, 454945091483900 . . . . . . . . . . . . . . . . . . . . . 103

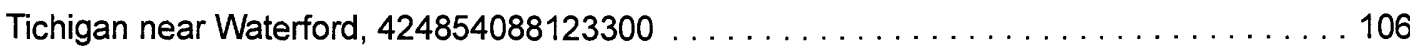
Twin

East Twin, near Westfield, 435430089350700 . . . . . . . . . . . . . . . . . . . . . . . . . 109

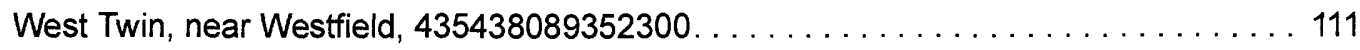

Waterford Waterway, Fox River Site, near Waterford, 424614088123600 . . . . . . . . . 113

Wazee, Deep Hole, near Black River Falls, 441730090432900 . . . . . . . . . . . . . . . . 116

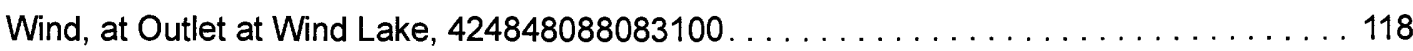

at Wind Lake, $424915088083900 \ldots \ldots$. . . . . . . . . . . . . . . . . . . . . . . . . . . 119

Winnebago

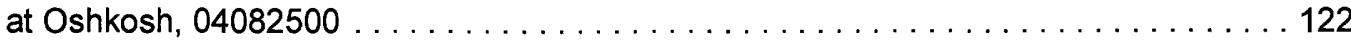

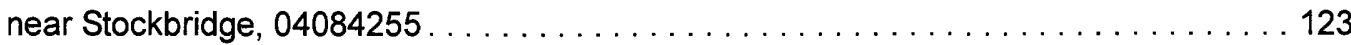

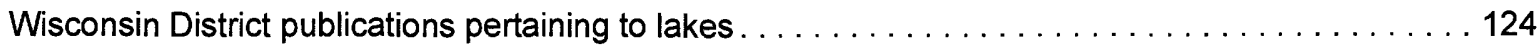

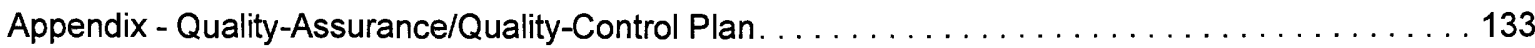




\section{CONTENTS--Continued}

\section{FIGURE}

Figure 1. Map showing location of lake water-quality and lake-stage stations in Wisconsin $\ldots \ldots \ldots 2$

\section{TABLES}

Table 1. Discontinued lake stations

2. Parameter identification numbers and laboratory reporting levels $(L R L)$ for chemical parameters commonly measured in lakes, and analyzed at the National Water-Quality Laboratory (NWQL) or the Wisconsin State Laboratory of Hygiene (WSLH) 
CONVERSION FACTORS, VERTICAL DATUM, AND ABBREVIATED WATER-QUALITY UNITS

\begin{tabular}{rcl}
\hline Multiply & By & To Obtain \\
\hline mile (mi) & 1.609 & kilometer \\
pound (lb) & 453.6 & gram \\
acre & 0.4048 & hectare \\
foot (ft) & 0.3048 & meter \\
gallon (gal) & 3.785 & liter \\
square mile $\left(\mathrm{mi}^{2}\right)$ & 2.590 & square kilometer
\end{tabular}

Temperature, in degrees Celsius $\left({ }^{\circ} \mathrm{C}\right)$ can be converted to degrees Fahrenheit $\left({ }^{\circ} \mathrm{F}\right)$ by use of the following equation:

$$
{ }^{\circ} \mathrm{F}=1.8\left({ }^{\circ} \mathrm{C}\right)+32 \text {. }
$$

Sea level: In this report "sea level" refers to the National Geodetic Vertical Datum of 1929 (NGVD of 1929)-a geodetic datum derived from a general adjustment of the first-order level nets of both the United States and Canada, formerly called Sea Level Datum of 1929.

Abbreviated water-quality units: Chemical concentrations and water temperature are given in metric units. Chemical concentration is given in milligrams per liter $(\mathrm{mg} / \mathrm{L})$ or micrograms per liter $(\mu \mathrm{g} / \mathrm{L})$. Milligrams per liter is a unit expressing the concentration of chemical constituents in solution as weight (milligrams) of solute per unit volume (liter) of water. One thousand micrograms per liter is equivalent to one milligram per liter. For water with dissolved-solids concentrations less than $7,000 \mathrm{mg} / \mathrm{L}$, the numerical values for concentrations expressed as $\mathrm{mg} / \mathrm{L}$ and $\mu \mathrm{g} / \mathrm{L}$ are the same as for concentrations in parts per million and parts per billion, respectively.

Specific conductance of water is expressed in microsiemens per centimeter at 25 degrees Celsius $(\mu \mathrm{S} / \mathrm{cm})$. This unit is equivalent to micromhos per centimeter at 25 degrees Celsius $(\mu \mathrm{mho} / \mathrm{cm})$, formerly used by the U.S. Geological Survey. 


\section{WATER-QUALITY AND LAKE-STAGE DATA FOR WISCONSIN LAKES, WATER YEAR 2004 By Wisconsin Water Science Center Lake- Studies Team}

\section{INTRODUCTION}

The U.S. Geological Survey (USGS), in cooperation with local and other agencies, collects data at selected lakes throughout Wisconsin. These data, accumulated over many years, provide a data base for developing an improved understanding of the water quality of lakes. To make these data available to interested parties outside the USGS, the data are published annually in this report series. The locations of water-quality and lake-stage stations in Wisconsin for water year 2004 are shown in figure 1. A water year is the 12-month period from October 1 through September 30 . It is designated by the calendar year in which it ends. Thus, the period October 1 , 2003 through September 30, 2004 is called "water year 2004."

The purpose of this report is to provide information about the chemical and physical characteristics of Wisconsin lakes. Data that have been collected at specific lakes, and information to aid in the interpretation of those data, are included in this report. Data collected include measurements of in-lake water quality and lake stage. Time series of Secchi depths, surface total phosphorus and chlorophyll a concentrations collected during non-frozen periods are included for all lakes. Graphs of vertical profiles of temperature, dissolved oxygen, $\mathrm{pH}$, and specific conductance are included for sites where these parameters were measured. Descriptive information for each lake includes: location of the lake, area of the lake's watershed, period for which data are available, revisions to previously published records, and pertinent remarks. Additional data, such as streamflow and water quality in tributary and outlet streams of some of the lakes, are published in another volume: "Water Resources Data-Wisconsin, 2004."

Water-resources data, including stage and discharge data at most streamflow-gaging stations, are available throught the World Wide Web on the Internet. The Wisconsin Water Science Center's home page is at http://wi.water.usgs.gov/. Information on the Wisconsin Water Science Center's Lakes Program is found at wi.water.usgs.gov/lake/index.html and wi.water.usgs.gov/projects/index.html. 


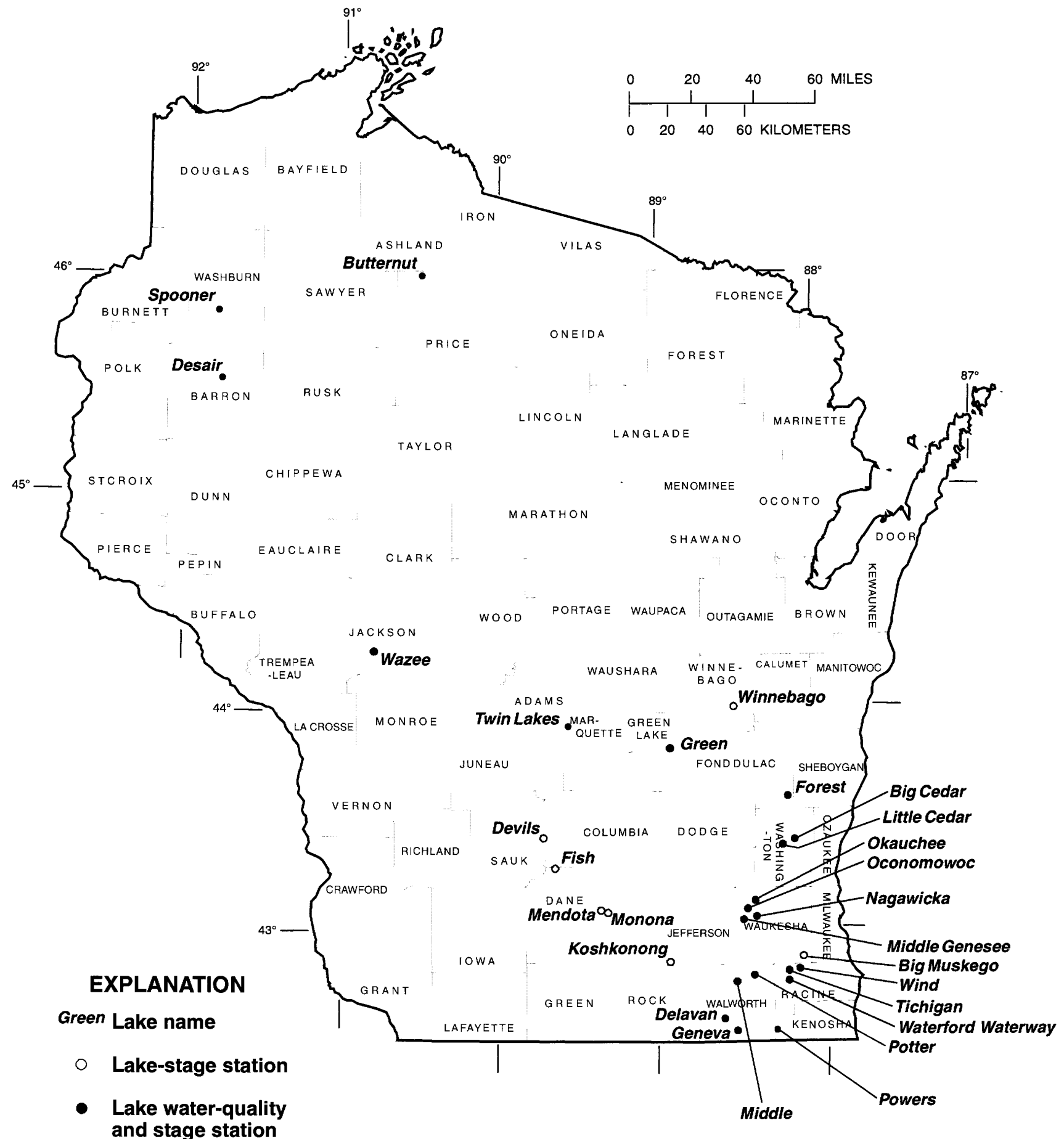

Note: at some lakes more than one site may be monitored.

Figure 1. Location of lake water-quality and lake-stage stations in Wisconsin. 
The USGS has done cooperative lake monitoring with local and other agencies since 1983. Cooperators in 2004 included:

Big Cedar Lake Protection and Rehabilitation District

City of Delafield

City of Muskego

Dane County Department of Public Works

Delavan Lake Sanitary District

Geneva Lake Environmental Agency

Green Lake Sanitary District

Jackson County

Lauderdale Lakes Lake District

Little Cedar Lake Protection and Rehabilitation District

Middle Genesee Lake District

Okauchee Lake Management District

Potters Lake Protection and Rehabilitation District

Powers Lake District

Rock County Public Works Department

Spooner Lake District

Town of Auburn (Forest Lake Association)

Town of Rice Lake (Desair Lake Restoration, Inc.)

Town of Springfield (Twin Lakes Conservancy)

U.S. Army Corps of Engineers

Village of Oconomowoc Lake

Waterford Waterway Management District

Wind Lake Management District

Wisconsin Department of Natural Resources 
Lake data-collection sites are identified by a unique identification number. Lake waterquality sites are identified by a 15-digit number that is a concatenation of the site's latitude, longitude, and a two-digit sequence number. The sequence number is used to distinguish between sites located at the same latitude-longitude designation. The site identification number is permanently assigned to the site; actual latitude and longitude of the site are subject to update and are stored separately. For some lakes, which have historical records of lake stage, an eightto-ten digit number is assigned according to downstream order. Gaps are left in the numerical series to allow for new stations; hence, the numbers are not consecutive. The first two digits of the complete eight-to-ten digit number, such as 04087000 or 054310157 , designate the major river basin. For example, "04" designates the St. Lawrence River Basin and "05" designates the Upper Mississippi River Basin.

The water-quality lake stations that were discontinued prior to water year 2004 are listed in table 1. Discontinued lake-stage stations are not included in this table.

This report is the culmination of a concerted effort by a number of people who collected, compiled, analyzed, verified, and organized the data, and who typed, edited, and assembled the report. The authors had primary responsibility for assuring that the information contained herein is accurate, complete, and adheres to USGS policy and established guidelines. Technicians in charge of the field offices are: T.J. Popowski (Rice Lake and Merrill), and S.A. March (Middleton). The data were collected and processed by S.M. Berg, C.J. Bloom, G.L. Goddard, D.E. Housner, S.B. Marsh, B.W. Olson, D.L. Olson, and J.G. Schuler. S.B. Marsh and P.A. Stark assembled, edited, and formatted the report. Additional assistance in preparation of the report was provided by M.M. Greenwood.

\section{METHODS OF DATA COLLECTION}

Depth profiles of water temperature, dissolved oxygen, $\mathrm{pH}$, and specific conductance were collected using multi-parameter meters. Prior to measurements, the meters were calibrated using standards for $\mathrm{pH}$ and conductance, and dissolved oxygen was calibrated using the air calibration

method. Generally, field measurements in profiles were made at $0.5-\mathrm{m}$ intervals if the maximum depth of the lake was $5 \mathrm{~m}$ or less and at 1.0-m intervals if the maximum depth was greater than $5 \mathrm{~m}$. 
Table 1. Discontinued lake stations

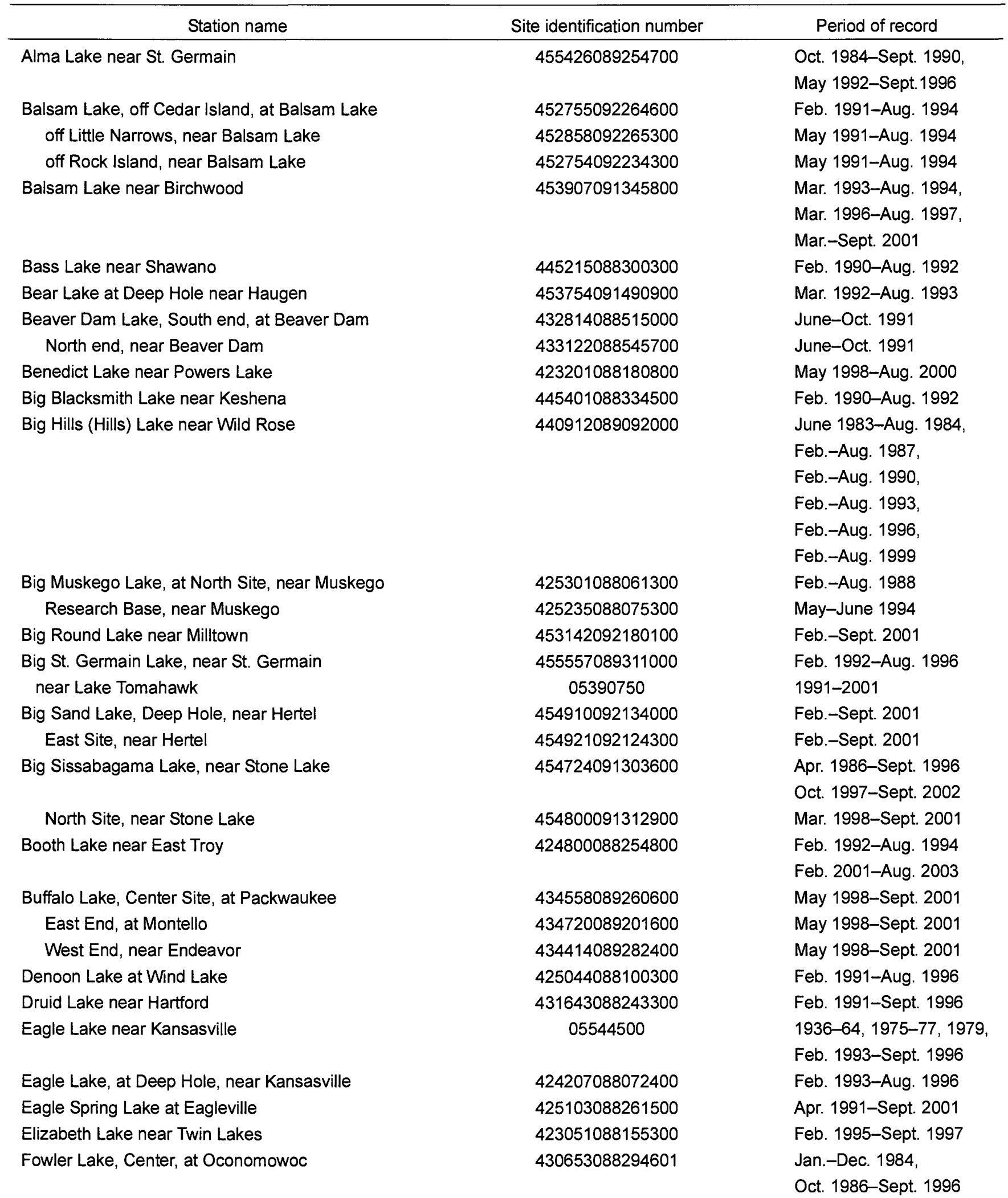


Table 1. Discontinued lake stations

\begin{tabular}{|c|c|c|}
\hline Station name & Site identification number & Period of record \\
\hline \multicolumn{3}{|l|}{ Geneva Lake } \\
\hline Williams Bay, at Williams Bay & 423420088320500 & Apr. 1997-Feb. 1999 \\
\hline Center, near Lake Geneva & 423402088301400 & Apr. 1997-Mar. 1999 \\
\hline Hemlock Lake near Mikana & & $\begin{array}{l}\text { Mar. 1996-Aug. 1997, } \\
\text { Mar.-Sept. } 2001\end{array}$ \\
\hline Hooker Lake at Salem & 423335088060300 & Feb. 1992-Aug. 1993 \\
\hline \multicolumn{3}{|l|}{ Kawaguesaga } \\
\hline (Site 1) near Cumberland & 453608092035801 & Nov. 1995-Nov. 1996 \\
\hline (Site 2) near Cumberland & 453601092035301 & Nov. 1995-Nov. 1996 \\
\hline (Site 3) near Cumberland & 453612092034901 & Nov. 1995-Nov. 1996 \\
\hline (Site 4) near Cumberland & 453603092035701 & Nov. 1995-Nov. 1996 \\
\hline (Site 5) near Cumberland & 453608092041201 & Nov. 1995-Nov. 1996 \\
\hline (Site 6) near Cumberland & 453555092040901 & Nov. 1995-Nov. 1996 \\
\hline \multirow[t]{3}{*}{ Lac La Belle at Oconomowoc } & 430733088305900 & Feb. 1984-Aug. 1985 \\
\hline & & Apr. -Aug. 1991 \\
\hline & & Feb. 2001-Aug. 2003 \\
\hline Lake Nebagamon, Northeast Bay, at Lake Nebagamon & 463050091412300 & May 1992-Aug. 1995 \\
\hline Southeast Bay, at Lake Nebagamon & 462928091413500 & Mar. 1992-Sept. 1995 \\
\hline West Bay, at Lake Nebagamon & 463034091425300 & May 1992-Aug. 1995 \\
\hline \multirow[t]{2}{*}{ Lake Noquebay near Crivitz } & 451511087550900 & Feb. 1987-Aug. 1988, \\
\hline & & Apr. 1991-Aug. 1994 \\
\hline East End, near Crivitz & 451540087525700 & Apr. 1991-Aug. 1994 \\
\hline Lamotte Lake near Shawano & 445305088361200 & Feb. 1990-Aug. 1992 \\
\hline \multicolumn{3}{|l|}{ Lauderdale Lakes } \\
\hline at Lauderdale & 424554088332700 & Oct. 1993-Oct. 1994 \\
\hline Green, Auxiliary, Number 1, near Lauderdale & 424640088341900 & June 1999-Sept. 2000 \\
\hline \multirow[t]{2}{*}{ Green, near Lauderdale } & 424652088341500 & Nov. 1993-Nov. 1994 \\
\hline & & Aug. 2002 \\
\hline \multirow[t]{2}{*}{ Mill, at Lauderdale } & 424555088335700 & Nov. 1993-Nov. 1994 \\
\hline & & Aug. 2002 \\
\hline Legend Lake (site 1) near Shawano & 445342088312700 & Feb. 1990-Feb. 1992 \\
\hline
\end{tabular}


Table 1. Discontinued lake stations

\begin{tabular}{|c|c|c|}
\hline Station name & Site identification number & Period of record \\
\hline Little Arbor Vitae near Woodruff & 455446089370300 & Feb. 1991-Sept. 2002 \\
\hline Little Green Lake, at Center, near Markesan & 434412088590700 & Feb. 1991-Aug. 2003 \\
\hline Little Muskego Lake at Muskego & 425425088083500 & Oct. 1986-Aug. 2002 \\
\hline Little Rock Lake near Woodruff & 455946089415702 & Oct. 1983-Sept. 1996 \\
\hline \multicolumn{3}{|l|}{ Little St. Germain Lake } \\
\hline near Eagle River & 05390700 & (a) \\
\hline \multirow[t]{3}{*}{ Upper East Bay, at St. Germain } & 455532089253900 & Dec. 1996-Mar. 97 \\
\hline & & Mar. 1999 \\
\hline & & Mar. 2000-Aug. 2003 \\
\hline \multirow[t]{3}{*}{ Northeast Bay, near St. Germain } & 455545089262500 & Apr. 1991-Aug. 1994 \\
\hline & & Aug. 1996-Aug. 1997 \\
\hline & & Mar. 1999-Aug. 2003 \\
\hline \multirow[t]{3}{*}{ South Bay, near St. Germain } & 455437089270800 & Apr. 1991-Aug. 1994 \\
\hline & & Aug. 1996-Aug. 1997 \\
\hline & & Mar. 1999-Aug. 2003 \\
\hline \multirow[t]{3}{*}{ West Bay, at St. Germain } & 455428089282400 & Apr. 1991-Aug. 1994 \\
\hline & & Aug. 1996-Aug. 1997 \\
\hline & & Mar. 1999-Aug. 2003 \\
\hline Little Sand Lake - Site No. 2 - near Mole Lake & 452826088544101 & May1996-Sept. 2003 \\
\hline \multirow[t]{2}{*}{ Long (Kee Nong Go-Mong) Lake at Wind Lake } & 424937088103400 & Feb. 1988-Aug. 1989, \\
\hline & & Feb. 1991-Aug. 1996 \\
\hline Loon Lake near Shawano & 445009088303700 & Feb. 1991-Aug. 1993 \\
\hline Lost Lake near Beaver Dam & 432640088580500 & June-Oct. 1991 \\
\hline \multicolumn{3}{|l|}{ McKenzie Lakes } \\
\hline \multicolumn{3}{|l|}{ McKenzie (Big McKenzie) } \\
\hline Deep Hole, near Spooner & 455507092013500 & Feb. 1987-Aug. 1998 \\
\hline Northern Site, near Spooner & 455540092022000 & June 1997-Aug. 1998 \\
\hline South Site, near Spooner & 455437092022300 & June 1997-Aug. 1998 \\
\hline Lower McKenzie, near Webb Lake & 455902092011900 & June 1997-Aug. 1998 \\
\hline Middle McKenzie, near Spooner & 455635092021800 & June 1997-Aug. 1998 \\
\hline Mary (Marie) Lake at Twin Lakes & 423128088151200 & Feb. 1995-Aug. 1997 \\
\hline Max Lake near Woodruff & 460128089423501 & Mar. 1988-Dec. 1996 \\
\hline Mead Lake, East Bay near Willard & 444720090445000 & Apr. 1991-Aug. 1995 \\
\hline West Bay near Willard & 444733090460100 & Feb. 1991-Sept. 1995 \\
\hline \multicolumn{3}{|l|}{ Minocqua Lake } \\
\hline Deep Hole, at Minocqua & 455214089412800 & May-Sept. 2003 \\
\hline North Bay, at Minocqua & 455232089424100 & May-Sept. 2003 \\
\hline South Bay, at Minocqua & 455206089425200 & May-Sept. 2003 \\
\hline Montello Lake at Montello & 434748089195800 & Feb. 1995-Aug. 1998 \\
\hline Moon Lake near St. Germain & 455504089260500 & Feb. 1992-Aug. 1996 \\
\hline Morgan Lake near Fence & 454622088324801 & Oct. 1987-Sept. 1998. \\
\hline Moshawquit Lake near Shawano & 445352088295800 & Feb. 1990-Aug. 1992 \\
\hline \multicolumn{3}{|l|}{ Muskego (Big Muskego) } \\
\hline Auxiliary Number 1, near Muskego & 425329088054000 & June 1996-Aug. 2000 \\
\hline
\end{tabular}


Table 1. Discontinued lake stations

\begin{tabular}{|c|c|c|}
\hline Station name & Site identification number & Period of record \\
\hline near Wind Lake & 425109088075000 & $\begin{array}{l}\text { Oct. 1987-Sept. 1989, } \\
\text { Jan. 1991-Sept. } 2002\end{array}$ \\
\hline South Site, near Muskego & 425212088072800 & Feb. 1988-Aug. 2002 \\
\hline Muskellunge Lake near Eagle River & 455700089224900 & June 2000-Aug. 2001 \\
\hline \multicolumn{3}{|l|}{ Namekagon Lakes } \\
\hline Garden, near Cable & 461224091033200 & Mar. 1998-Aug. 1999 \\
\hline Jackson, near Cable & 461457091065900 & Mar. 1998-Aug. 1999 \\
\hline \multicolumn{3}{|l|}{ Namekagon } \\
\hline Park Lake (site 1) at Pardeeville & 433239089175800 & $\begin{array}{l}\text { Feb. 1986-Aug. } 1987 \text {, } \\
\text { May-Nov. } 1993\end{array}$ \\
\hline (site 2) at Pardeeville & 433226089175500 & May-Nov. 1993 \\
\hline (site 3 ) at Pardeeville & 433245089173000 & May-Nov. 1993 \\
\hline (site 4) at Pardeeville & 433257089165100 & May-Nov. 1993 \\
\hline Pike Lake near Hartford & 431916088200501 & Dec. 1998-Dec. 2000 \\
\hline Pike Lake-QW Site-near Hartford & 431835088200600 & Feb.-Aug. 2000 \\
\hline Pretty Lake, at Deep Hole, near Dousman & 425722088295000 & Feb. 1993-Aug. 1997 \\
\hline Red Cedar Lake, at Mikana & 453522091360600 & $\begin{array}{l}\text { Mar. 1993-Aug. 1994, } \\
\text { Mar. 1996-Aug. 1997, } \\
\text { Oct. 2000-Sept. } 2001\end{array}$ \\
\hline Rice Lake at Deep Hole near Whitewater & 424629088415700 & Apr.-Nov. 1991 \\
\hline Round Lake near Shawano & 445328088335000 & Feb. 1990-Aug. 1992 \\
\hline Sand Lake (Deep Hole) near Keshena & 445321088323101 & June-Aug. 1992 \\
\hline Shell Lake at Shell Lake & 05334000 & Aug. 1936-Sept. 1999 \\
\hline Silver Lake near Oconomowoc & 430436088293300 & Apr. 1992-Aug. 1996 \\
\hline Silver Lake near West Bend & 432322088125000 & Feb. 1996-Aug. 1997 \\
\hline Sinissippi Lake, off Anthony Is., at Hustisford & 432113088361100 & Feb. 1991-Aug. 1993 \\
\hline off Butternut Is., near Hustisford & 432240088363900 & Apr. 1991-Aug. 1993 \\
\hline off Sam Point, near Hustisford & 432300088374200 & Apr. 1991-Aug. 1993 \\
\hline Spirit Lake near Keshena & 445400088320100 & Apr.-Aug. 1992 \\
\hline Stewart Lake at Mt. Horeb & 430117089442701 & May 1992-Sept. 1993 \\
\hline Tombeau Lake near Powers Lake & 423153088184800 & May 1998-Aug. 2000 \\
\hline Upper Nemahbin Lake, Center, near Delafield & 430400088254900 & June 1993-Aug. 1995 \\
\hline
\end{tabular}


Table 1. Discontinued lake stations

\begin{tabular}{lcl}
\hline \multicolumn{1}{c}{ Station name } & Site identification number & Period of record \\
\hline \multicolumn{1}{c}{ South Site, near Delafield } & 430339088254800 & June 1993-Aug. 1995 \\
Outlet near Delafield & 430334088255400 & June 1993-Aug. 1995 \\
Vandercook Lake near Woodruff & 455909089405602 & Nov. 1980-Aug. 1998 \\
Watosah-skice Lake near Keshena & 445330088361400 & Feb. 1990-Aug. 1992 \\
Waubeesee Lake at Wind Lake & 424857088101500 & Feb. 1988-Aug. 1989, \\
& & Feb. 1991-Aug. 1996 \\
Wazee Lake & & \\
Beach, near Black River Falls & 441721090431700 & Nov. 1999-Aug. 2000 \\
& & June-Sept. 2003 \\
Whitefish Lake, North Basin, near Gordon & 461321091520900 & Mar. 1998-Sept. 2001 \\
South Basin, near Gordon & 461212091523200 & Mar. 1998-Sept. 2001 \\
Whitewater Lake, off Heart Prairie, near Whitewater & 424533088420100 & Apr.-Nov. 1991 \\
near Whitewater & 424608088414800 & Nov. 1990-Sept. 2002 \\
North Bay, near Whitewater & 424625088405500 & Apr.-Nov. 1991 \\
South Bay, near Whitewater & 424501088422300 & Apr.-Nov. 1991 \\
Wind Lake, Northeast Basin, at Wind Lake & 424938088080800 & Feb. 1997-Aug. 1998 \\
Wolf Lake near Mt. Calvary & 435152088123100 & Nov. 1983-Sept. 1986, \\
& & Nov. 1992-Sept. 1997 \\
& & \\
\hline
\end{tabular}


In most lakes, water samples were collected at two depths - near the surface and near the bottom. Chemical analyses of water samples were performed using standard analytical methods by either the USGS National Water Quality Laboratory (Wershaw and others, 1987; Fishman and Friedman, 1989; Fishman, 1993) or the Wisconsin State Laboratory of Hygiene (Wisconsin State Laboratory of Hygiene, 1993). Analyses for dissolved constituents were performed on samples that were filtered in the field through a $0.45-\mu \mathrm{m}$ (micrometer) pore-size filter. Total or total recoverable constituents were determined by analyzing unfiltered water samples. Preservation and shipment of samples followed standard protocols established by the laboratories. Water-quality data were archived in the Water Quality Data Base (QWDATA) of the National Water Information System (NWIS). Additional descriptive information about water-quality data is available in the data report: "Water Resources Data - Wisconsin, 2004". NWIS parameter codes and minimum laboratory reporting levels for chemical constituents are given in table 2.

Records of lake stage are considered complete when one or more manual or automatic measurements were obtained per day. Partial records of lake stage result when measurements were less frequent than daily. A complete description of manual or automatic measurements of lake stage is described by Rantz and others (1982).

\section{EXPLANATION OF PHYSICAL AND CHEMICAL CHARACTERISTICS OF LAKES}

Following are brief, generalized explanations of some of the common measurements of water quality and some of the physical processes occurring in lakes that influence these measures of water quality. More detailed explanations of water-quality data and lake processes are given by Wetzel (1983), Hem (1985), and Shaw and others (1993).

\section{Water Temperature and Thermal Stratification}

Water temperature in lakes is important because of its role in stratification and because of the temperature dependence of many chemical reactions and life processes of aquatic organisms. The extent of thermal stratification in lakes depends on the interaction between the lake's shape, water clarity, solar heating, and wind-driven mixing. Complete mixing of the lake is usually inhibited by thermal stratification in summer and by ice cover in winter. Thermal stratification affects water quality and the distribution of organisms in the lake. Summer thermal stratification can occur in any lake, but in Wisconsin it commonly occurs in lakes deeper than about $6 \mathrm{~m}$ (Shaw and others, 1993). 
Table 2. Parameter identification numbers and laboratory reporting levels (LRL) for chemical parameters commonly measured in lakes, and analyzed at the National Water Quality Laboratory (NWQL) or the Wisconsin State Laboratory of Hygiene (WSLH)

\begin{tabular}{|c|c|c|c|c|c|c|c|c|c|}
\hline \multirow[b]{3}{*}{ Parameter Name } & \multirow[b]{3}{*}{ Units } & \multirow[b]{3}{*}{$\begin{array}{l}\text { CAS } \\
\text { Number } \\
\text { (1) }\end{array}$} & \multirow[b]{3}{*}{$\begin{array}{l}\text { Parameter } \\
\text { Code } \\
\text { (2) }\end{array}$} & \multicolumn{4}{|c|}{ (NWQL) } & \multicolumn{2}{|c|}{ (WSLH) } \\
\hline & & & & \multicolumn{2}{|c|}{ Standard Analysis } & \multicolumn{2}{|c|}{ Low-Level Analysis } & \multirow[b]{2}{*}{ LRL } & \multirow[b]{2}{*}{$\begin{array}{c}\text { Test } \\
\text { Code }\end{array}$} \\
\hline & & & & LRL & $\begin{array}{l}\text { Lab } \\
\text { Code }\end{array}$ & LRL & $\begin{array}{l}\text { Lab } \\
\text { Code }\end{array}$ & & \\
\hline Calcium, diss. (Ca) & $\mathrm{mg} / \mathrm{L}$ & $7440-70-2$ & 00915 & 0.020 & 659 & 0.002 & 1895 & 0.02 & I230IUD \\
\hline Magnesium, diss. (Mg) & $\mathrm{mg} / \mathrm{L}$ & $7439-95-4$ & 00925 & 0.004 & 663 & 0.001 & 1897 & 0.02 & I390IUD \\
\hline Sodium, diss. (Na) & $\mathrm{mg} / \mathrm{L}$ & $7440-23-5$ & 00930 & 0.09 & 675 & 0.025 & 1898 & 0.09 & I80IUD \\
\hline Potassium, diss. (K) & $\mathrm{mg} / \mathrm{L}$ & $7440-09-7$ & 00935 & 0.24 & 54 & 0.01 & 833 & 0.3 & I540IUD \\
\hline Sulfate, diss. $\left(\mathrm{SO}_{4}\right)$ & $\mathrm{mg} / \mathrm{L}$ & $14808-79-8$ & 00945 & 0.31 & 1572 & 0.01 & 1263 & 1.0 & I600DLD \\
\hline Chloride, diss. (Cl) & $\mathrm{mg} / \mathrm{L}$ & $16887-00-6$ & 00940 & 0.29 & 1571 & 0.01 & 1259 & 0.1 & I240ELD \\
\hline Fluoride, diss. (F) & $\mathrm{mg} / \mathrm{L}$ & $16984-48-8$ & 00950 & 0.100 & 31 & 0.01 & 1260 & 0.03 & I330FLD \\
\hline Iron, diss. (Fe) & $\mu g / L$ & $7439-89-6$ & 01046 & 10 & 645 & 3 & 1896 & 10 & I370IUD \\
\hline Manganese, diss. (Mn) & $\mu g / L$ & $7439-96-5$ & 01056 & 2.2 & 648 & 1 & 1793 & 0.4 & |400IUD \\
\hline Silica, diss. $\left(\mathrm{SiO}_{2}\right)$ & $\mathrm{mg} / \mathrm{L}$ & $7631-86-9$ & 00955 & 0.1 & 56 & 0.02 & 1899 & 0.008 & I560LLD \\
\hline Nitrogen, $\mathrm{NO}_{2}+\mathrm{NO}_{3}$, diss. & $\mathrm{mg} / \mathrm{L}$ & & 00631 & 0.05 & 1975 & 0.005 & 1979 & 0.01 & I460MLD \\
\hline Nitrogen, ammonia, diss. & $\mathrm{mg} / \mathrm{L}$ & $7664-41-7$ & 00608 & 0.02 & 1976 & 0.002 & 1980 & 0.013 & I440NLD \\
\hline Nitrogen, organic, total (3) & $\mathrm{mg} / \mathrm{L}$ & & & & & & & & \\
\hline Nitrogen, amm.+org., total (4) & $\mathrm{mg} / \mathrm{L}$ & $17778-88-0$ & 00625 & 0.100 & 1985 & & & 0.2 & 1470BLT \\
\hline Nitrogen, amm.+org.,diss. & $\mathrm{mg} / \mathrm{L}$ & & 00623 & & & & & & I470DLD \\
\hline Nitrogen, total (5) & $\mathrm{mg} / \mathrm{L}$ & & & & & & & & \\
\hline Nitrogen, dissolved & $\mathrm{mg} / \mathrm{L}$ & & 00602 & & & & & & \\
\hline Phosphorus, total & $\mathrm{mg} / \mathrm{L}$ & $7723-14-0$ & 00665 & 0.05 & 1984 & 0.004 & 2333 & 0.005 & I520PLT \\
\hline Phosphorus, ortho, diss. & $\mathrm{mg} / \mathrm{L}$ & $14265-44-2$ & 00671 & 0.01 & 1262 & 0.002 & 1978 & 0.002 & 1530CLD \\
\hline Chlorophyll a, phytoplankton & $\mu \mathrm{g} / \mathrm{L}$ & $479-61-8$ & 70953 & 0.1 & 586 & & & & \\
\hline Chlorophyll a, phytoplankton & $\mu g / L$ & $479-61-8$ & 32210 & & & & & 0.26 & 1250UNF \\
\hline
\end{tabular}

Footnotes:

1: CAS (Chemical Abstracting Services) number = unique identification for each constituent

2: Parameter Code - unique number for storage of data in database

3: Calculated as difference between total ammonia + organic nitrogen and ammonia nitrogen

4: Also known as Total Kjeldahl Nitrogen (TKN)

5: Calculated as sum of TKN + Nitrogen as $\left(\mathrm{NO}_{2}+\mathrm{NO}_{3}\right)$ 
The density of water increases with decreasing temperature down to a temperature of $4^{\circ} \mathrm{C}$, then decreases with decreasing temperature between $4^{\circ} \mathrm{C}$ and the freezing point of water $\left(0^{\circ} \mathrm{C}\right)$. For a brief period in the spring after the ice is out, water temperature is usually uniform through the entire water column and wind action causes the lake to mix completely. This process is known as "spring turnover." As the lake absorbs the sun's energy, the surface water becomes warmer and its density decreases, making it more resistant to complete mixing. The difference in density caused by different water temperatures can prevent warm and cold water from mixing. In most lakes, therefore, a density "barrier" forms between the warmer surface water (epilimnion) and the underlying colder water (hypolimnion). This barrier is often marked by a sharp temperature gradient known as the "thermocline (metalimnion)." During the stratified summer period, these three distinct layers of lake water are often present. As the temperature difference between surface and deep water increases, this "stratified" condition stabilizes and can persist until surface temperatures decrease in the fall, which decreases the stability of the stratification. The mixing of the lake water in the fall is known as "fall turnover."

Thermal stratification may also occur under ice cover in the winter. In the winter, the coldest water (near $0^{\circ} \mathrm{C}$ ) under the ice at the surface of the lake is less dense than water deeper in the lake with warmer temperatures.

\section{Specific Conductance}

Specific conductance is a measure of the ability of water to conduct an electrical current and is an indicator of the concentration of dissolved solids in the water. Because conductance is temperature related, reported values are normalized at $25^{\circ} \mathrm{C}$ and are termed specific conductance. As the concentration of dissolved minerals increases, specific conductance increases. During winter and summer thermal stratification, concentrations of dissolved constituents near the lake bottom increase due to the decomposition of materials settling from the epilimnion, or release of dissolved materials (such as iron, manganese, and phosphorus) from the bottom sediments during anoxic periods. Therefore, differences in specific conductance with depth indicate differences in concentrations of dissolved solids. 


\section{Water Clarity}

Water clarity, or transparency, is commonly measured using a Secchi disc. The range of depths within which photosynthetic activity occurs depends largely on depth of light penetration, which is influenced by water clarity. A Secchi disc, most commonly an $20-\mathrm{cm}$.-diameter disc with alternating black-and-white quadrants, is lowered to a depth at which it is no longer visible. This depth is referred to as the Secchi depth. Clarity can be reduced by algae, zooplankton, water color, and suspended sediment. Algae are often the most dominant influence on clarity in lakes and, therefore, Secchi depth is usually correlated with the algal abundance. Secchi depths are generally the least during summer when algal populations are largest.

\section{pH}

The $\mathrm{pH}$ is a measure of the acidity of the water. It is defined as the negative logarithm of hydrogen-ion concentration and varies over a 14-unit log scale, with a pH of 7 being neutral. Values less than 7 indicate acidic conditions; the lower the value, the stronger the acidity. Values greater than 7 indicate alkaline conditions. The $\mathrm{pH}$ of water is influenced in part by photosynthesis and respiration of planktonic algae and aquatic plants. It is important because it affects the solubility of many chemical constituents, and because aquatic organisms have limited pH tolerances. Planktonic algae and aquatic plants produce oxygen and consume carbon dioxide as they photosynthesize during daytime; they consume oxygen and produce carbon dioxide when they respire at night. Carbon dioxide combines with the water molecule to form carbonic acid; therefore respiration causes a decrease in $\mathrm{pH}$ at night and photosynthesis during the day causes an increase in $\mathrm{pH}$. The result is a daily cycle in $\mathrm{pH}$. Because phytoplankton are usually concentrated in the near-surface water, changes in $\mathrm{pH}$ in the epilimnion are more extreme than in the hypolimnion, where less photosynthesis usually occurs.

Lakes having good fish populations and productivity generally have a pH between 6.7 and 8.2. Values of $\mathrm{pH}$ greater than 8.5 have been shown to cause the release of phosphorus from lake sediments (James and Barko, 1991). 


\section{Dissolved Oxygen}

Dissolved oxygen is one of the most critical factors affecting a lake ecosystem because it is essential to most aquatic organisms, and it is involved in many chemical reactions. Very low dissolved oxygen concentrations can control some types of chemical reactions. The solubility of oxygen in water is inversely related to temperature-that is, oxygen solubility decreases as water temperature increases. This relation is important because at warmer temperatures the metabolic rate of organisms increases but less oxygen is available for respiration. The primary sources of dissolved oxygen are from the air and from photosynthesis. The minimum dissolved oxygen concentration specified in national water-quality criteria for early life stages of warmwater aquatic life is 5.0 mg/L (U.S. Environmental Protection Agency, 1986).

In early summer, if thermal stratification develops, the metalimnion restricts the surface supply of dissolved oxygen to the hypolimnion. The hypolimnion can become isolated from the atmosphere. Thus, as summer progresses, the dissolved oxygen concentration can decrease in response to decomposition of dead algae that settle from the epilimnion and in response to the biological and chemical oxygen demand of the sediments. The oxygen demand from these processes may completely deplete the oxygen (anoxia) in the water near the lake bottom. The oxygen depletion then progresses upward but usually is confined to the hypolimnion.

Anoxia in the hypolimnion is common in stratified eutrophic (nutrient-rich) lakes in Wisconsin. Complete anoxia, however, is often not detected because of meter constraints. During anoxic conditions, many aquatic organisms cannot survive, but many other species (primarily bacteria) actually function only in such conditions. Therefore, a shift from oxic to anoxic conditions produces a rapid and dramatic change in the biological community and chemical environment. Anoxia also can cause

release of phosphorus from the bottom sediments. This phosphorus then mixes throughout the water column during spring and fall turnover. 


\section{Phosphorus}

Phosphorus is one of the essential nutrients for plant growth. High phosphorus concentrations can cause dense algal populations (blooms) and can therefore be a major cause of eutrophication in lakes. When phosphorus concentrations exceed $0.025 \mathrm{mg} / \mathrm{L}$ at the time of spring overturn in lakes and reservoirs, these water bodies may occasionally experience excess or nuisance growth of algae or other aquatic plants (U.S. Environmental Protection Agency, 1986). In many regions of the country, including the upper Midwest, other nutrients, particularly nitrogen, tend to be in abundant supply. Phosphorus is often the nutrient in shortest supply, therefore limiting or controlling plant growth. About 90 percent of the lakes in Wisconsin are limited by phosphorus (Shaw and others, 1993). In water, dissolved orthophosphate is that part of total phosphorus that is most readily available for use by algae.

Internal phosphorus recycling occurs in many lakes. Phosphorus used by algae, aquatic plants, fish, and zooplankton is stored within these organisms. As these organisms die and decompose, this phosphorus is returned to the lake water and sediments. Anoxia in the hypolimnion makes phosphorus more soluble, adding further to the release of phosphorus from the falling particles and the lake sediments. During spring and fall turnover the phosphorus, which was released from the bottom sediments into the hypolimnion during anoxia, is mixed throughout the lake. The phosphorus is then available for algal growth. These phenomena are part of the internal-recycling processes of lakes.

\section{Nitrogen}

Nitrogen, like phosphorus, is an essential nutrient for plant and algal growth. Usually in Wisconsin lakes, nitrogen is in abundant supply from the atmosphere and other sources. If phosphorus is abundant relative to algal needs, nitrogen can become the limiting nutrient. In that case, algal blooms are more likely to be triggered by increases in nitrogen than by increases in phosphorus. Some bluegreen algal species can fix nitrogen from the atmosphere (Wetzel, 1983). Therefore, in situations where other types of algae are excluded because of a shortage of nitrogen, the nitrogen-fixing bluegreen algae have a competitive advantage and may be present in abundance. 
Lakes with a nitrogen to phosphorus ratio larger than 15 to 1 near the surface may generally be considered phosphorus limited; a ratio from 10 to 1 to 15 to 1 indicates a transition situation; and a ratio smaller than 10 to 1 generally indicates nitrogen limitation. Total nitrogen is the sum of ammonia, organic nitrogen, and nitrate-plus-nitrite nitrogen. The near-surface concentration is commonly used to compute the total nitrogen to phosphorus ratio because most algal species grow near the lake surface.

\section{Chlorophyll a}

Chlorophyll $a$ is a photosynthetic pigment found in algae (Wetzel, 1983) and other green plants. Its concentration, therefore, is commonly used as a measure of the density of the algal population in a lake. Chlorophyll a concentrations are generally highest during summer when algal populations are highest. Moderate populations of desirable algae are important in the food chain; however, excessive populations or algal blooms are undesirable. Algal blooms can cause taste and odor problems, and limit light penetration needed to support growth of submerged aquatic plants. Certain species of bluegreen algae can produce toxins (Rapavich and others, 1987).

\section{CLASSIFICATION OF LAKES}

Two methods are commonly used to classify and evaluate Wisconsin lakes according to their water quality or trophic state: Lillie and Mason's (1983) water-quality index and a modification of Carlson's (1977) Trophic State Index (TSI) by Lillie and others (1993). Three waterquality measures are used in these classification systems: near-surface concentrations of total phosphorus and chlorophyll $a$, and water clarity as indicated by the Secchi depth.

Lillie and Mason's (1983) water-quality indices for Wisconsin lakes were developed based on random summer measurements of total phosphorus and chlorophyll a concentrations, and Secchi depth to classify the lakes' water quality as shown below:

\begin{tabular}{lccc}
\hline $\begin{array}{c}\text { Water-quality } \\
\text { index }\end{array}$ & $\begin{array}{c}\text { Total phosphorus range } \\
(\mathrm{mg} / \mathrm{L})\end{array}$ & $\begin{array}{c}\text { Chlorophyll a range } \\
(\mu \mathrm{g} / \mathrm{L})\end{array}$ & $\begin{array}{c}\text { Water clarity range } \\
(\text { Secchi depth, in meters })\end{array}$ \\
\hline "Excellent" & $<0.001$ & $<1.0$ & $>6.0$ \\
"Very good" & $.001-.009$ & $1.0-4.9$ & $3.0-6.0$ \\
"Good" & $.010-.029$ & $5.0-9.9$ & $2.0-2.9$ \\
"Fair" & $.030-.049$ & $10.0-14.9$ & $1.5-1.9$ \\
"Poor" & $.050-.149$ & $15.0-30.0$ & $1.0-1.4$ \\
"Very poor" & $>.150$ & $>30.0$ & $<1.0$ \\
\hline
\end{tabular}


The TSI approach to lake classification assigns numerical ranges to the three trophic conditions generally used to describe the wide range of lake water-quality conditions. Oligotrophic lakes are typically clear, algal populations and phosphorus concentrations are low, and the deepest water is likely to contain oxygen throughout the year. Mesotrophic lakes typically have a moderate supply of nutrients, experience moderate algal blooms, and have occasional oxygen depletions at depth. Eutrophic lakes are nutrient rich with relatively severe water-quality problems, such as frequent seasonal algal blooms, oxygen depletion in lower parts of the lakes, and poor clarity. When eutrophic conditions are very severe, the lake is considered hypereutrophic.

The WDNR modified the lakes classification scheme developed by Carlson (1977) to apply specifically to Wisconsin lakes. The WDNR system (Lillie and others, 1993) uses surface total phosphorus and chlorophyll a concentrations, and Secchi depth for ice-free periods to calculate values for TSI's. The WDNR has adopted the following TSI ranges to classify Wisconsin lakes: indices of less than 40 define oligotrophic conditions, 40 to 50 define mesotrophic conditions, greater than 50 to define eutrophic conditions, and greater than 70 define hypereutrophic conditions (Wisconsin Department of Natural Resources, 1992). These ranges are used to make relative comparisons in Wisconsin lake trophic-state evaluations by the WDNR and others.

The TSI for a lake can be calculated using the following equations (Lillie and others, 1993):

$T S I_{\text {Secchi }}=60.0-33.2 \times\left(\log _{10}\right.$ Secchi depth $)$

$\mathrm{TSI}_{\text {chlorophyll a }}=34.82+\left(17.41 \times\left(\log _{10}\right.\right.$ chlorophyll a concentration $\left.)\right)$

$T S I_{\text {total phosphorus }}=28.24+\left(17.81 \times\left(\log _{10}\right.\right.$ [total phosphorus concentration $\left.\left.\left.\times 1,000\right]\right)\right)$

where: Secchi depth is in meters, chlorophyll $a$ is in micrograms per liter, and total phosphorus is in milligrams per liter.

The three trophic conditions are defined with the following boundaries for total phosphorus, Secchi disc, and chlorophyll a:

\begin{tabular}{ccccc}
\hline $\begin{array}{c}\text { Trophic } \\
\text { Level }\end{array}$ & $\begin{array}{c}\text { Trophic State } \\
\text { Index }\end{array}$ & $\begin{array}{c}\text { Total phosphorus } \\
(\mathrm{mg} / \mathrm{L})\end{array}$ & $\begin{array}{c}\text { Secchi depth } \\
(\mathrm{m})\end{array}$ & $\begin{array}{c}\text { Chlorophyll a } \\
(\mu \mathrm{g} / \mathrm{L})\end{array}$ \\
\hline
\end{tabular}

Eutrophic 50 0.017 $-2.0$ $-7.4$

Mesotrophic 40 0.005 4.0 $-2.0$

Oligotrophic 


\section{REFERENCES CITED}

Carlson, R.E., 1977, A trophic state index for lakes: Limnology and Oceanography, March, v. 22, no. 2 , p. 361-369.

Fishman, M.J., ed., 1993, Methods of analysis by the U.S. Geological Survey National Water Quality Laboratory-Determination of inorganic and organic constituents in water and fluvial sediments: U.S. Geological Survey Open-File Report 93-125, 217 p.

Fishman, M.J., and Friedman, L.C., eds., 1989, Methods for determination of inorganic substances in water and fluvial sediments (3d ed.): U.S. Geological Survey Techniques of Water-Resources Investigations, book 5, chap. A1, 545 p.

Hem, J.D., 1985, Study and interpretation of the chemical characteristics of natural water (3rd ed.): U.S. Geological Survey Water-Supply Paper 2254, 263 p.

James, W.F., and Barko, J.W., 1991, Littoral-pelagic phosphorus dynamics during nighttime convective circulation: Limnology and Oceanography, v. 36, no. 5, p. 946-960.

Lillie, R.A., Graham, S., and Rasmussen, P., 1993, Trophic-State Index equations and regional predictive equations for Wisconsin lakes: Wisconsin Department of Natural Resources Research Management Findings No. 35, 4 p.

Lillie, R.A., and Mason, J.W., 1983, Limnological characteristics of Wisconsin lakes: Wisconsin Department of Natural Resources Technical Bulletin No. 138, 116 p.

Rantz, S.E., and others, 1982, Measurement and computation of streamflow: U.S. Geological Survey Water-Supply Paper 2175, 631 p.

Rapavich, W.M., Sonzogni, W.C., Standridge, J.H., Vennie J.G., and Wedepohl, R.E., 1987, Incidence of algal toxins in Wisconsin water experiencing blue-green algae blooms: Wisconsin State Laboratory of Hygiene and Wisconsin Department of Natural Resources, Informational Paper, $8 \mathrm{p}$.

Shaw, B., Mechenich, C., and Klessig, L., 1993, Understanding Lake Data: G3582: University of Wisconsin Extension, Madison, Wis., 19 p.

U.S. Environmental Protection Agency, 1986, Quality Criteria for Water 1986: U.S. Environmental Protection Agency publication, EPA 440/5-86-001 [variously paged].

Wershaw, R.L., Fishman, M.J., Grabbe, R.R., and Lowe, L.E., eds., 1987, Methods for the determination of organic substances in water and fluvial sediments: U.S. Geological Survey Techniques of Water-Resources Investigations, book 5, chap. A3, 80 p.

Wetzel, R.G., 1983, Limnology (2d ed.): New York, W.B. Saunders, 767 p. 
Wisconsin Department of Natural Resources, 1992, Wisconsin water quality assessment-Report to Congress, 1992: Wisconsin Department of Natural Resources Publ-WR254-92-REV, $220 \mathrm{p}$.

Wisconsin State Laboratory of Hygiene, Environmental Sciences Section, 1993, Manual of analytical methods, inorganic chemistry unit: Wisconsin State Laboratory of Hygiene, revised November 1993 [variously paged]. 


\section{LAKE DATA}




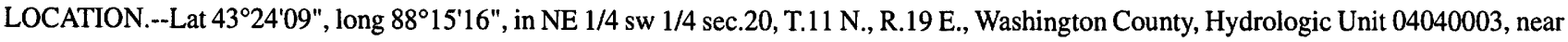
West Bend.

PERIOD OF RECORD.--February 2000 to to current year.

REMARKS.--Lake sampled on north side at a depth of $12 \mathrm{~m}$. Lake ice-covered during February sampling. Water-quality analyses by Wisconsin State Laboratory of Hygiene.

WATER-QUALITY DATA, FEBRUARY 24 TO AUGUST 18, 2004

(Milligrams per liter unless otherwise indicated)

Date

Secchi depth $(\mathrm{m})$

Depth of sample $(\mathrm{m})$

Chlorophyll a, phytoplankton $(\mu \mathrm{g} / \mathrm{L})$

Water temperature $\left({ }^{\circ} \mathrm{C}\right)$

Specific conductance $(\mu \mathrm{S} / \mathrm{cm})$

$\mathrm{pH}$

Dissolved oxygen (mg/L)

Phosphorus, total (as P)

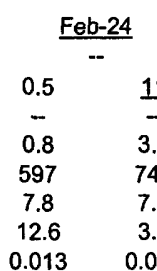

2-24-04
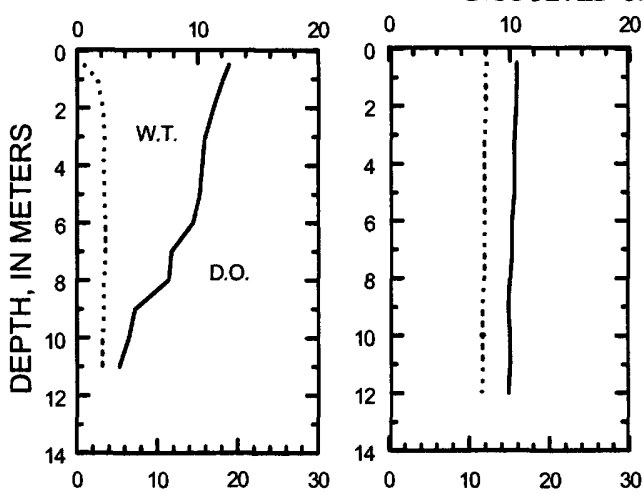

4-22-04

6-9-04

7-14-04

\begin{tabular}{ccc}
\multicolumn{2}{c}{$\frac{\text { Jun-9 }}{3.1}$} & \\
0.5 & 11.5 & 0.5 \\
4.5 & - & 9.5 \\
24.1 & 12.5 & 24.1 \\
516 & 557 & 514 \\
8.2 & 7.5 & 8.2 \\
10.6 & 0.4 & 11.6 \\
0.015 & 0.026 & 0.014
\end{tabular}

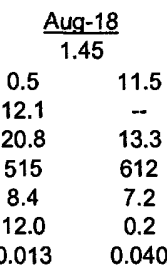

DISSOLVED OXYGEN (D.O.) IN MILIGRAMS PER LITER
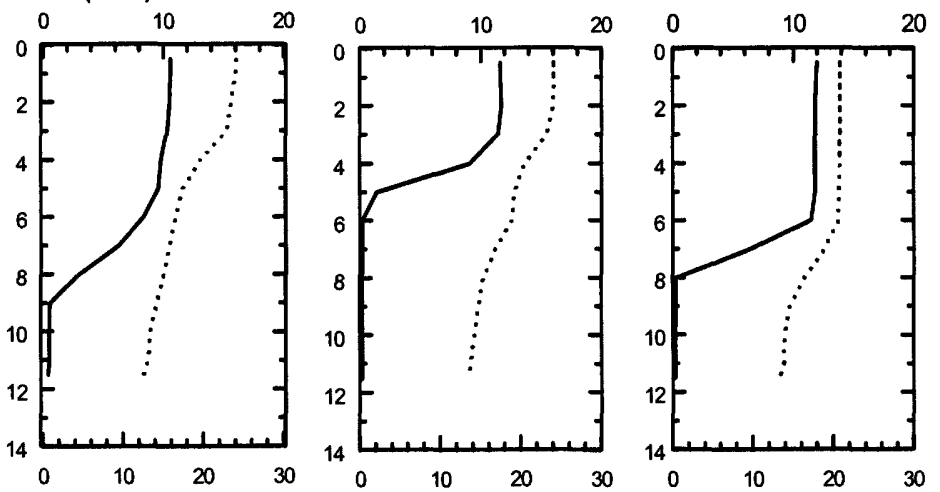

WATER TEMPERATURE (W.T.) IN DEGREES CELSIUS

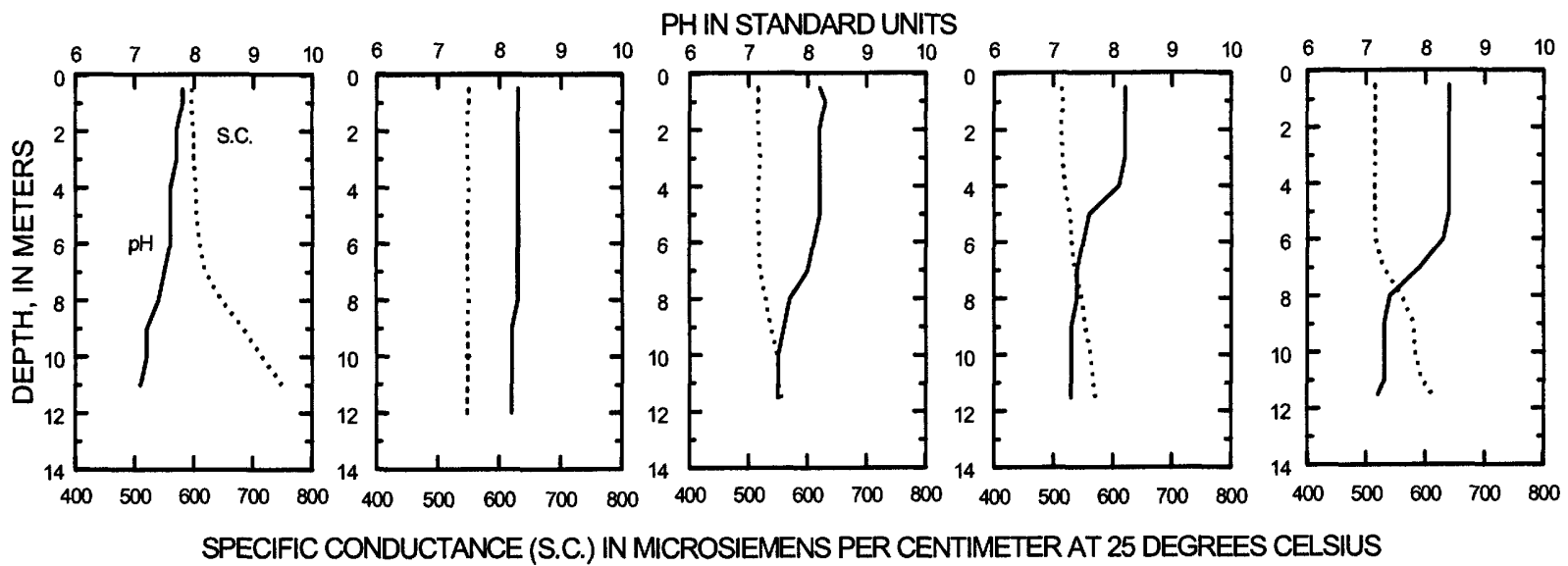



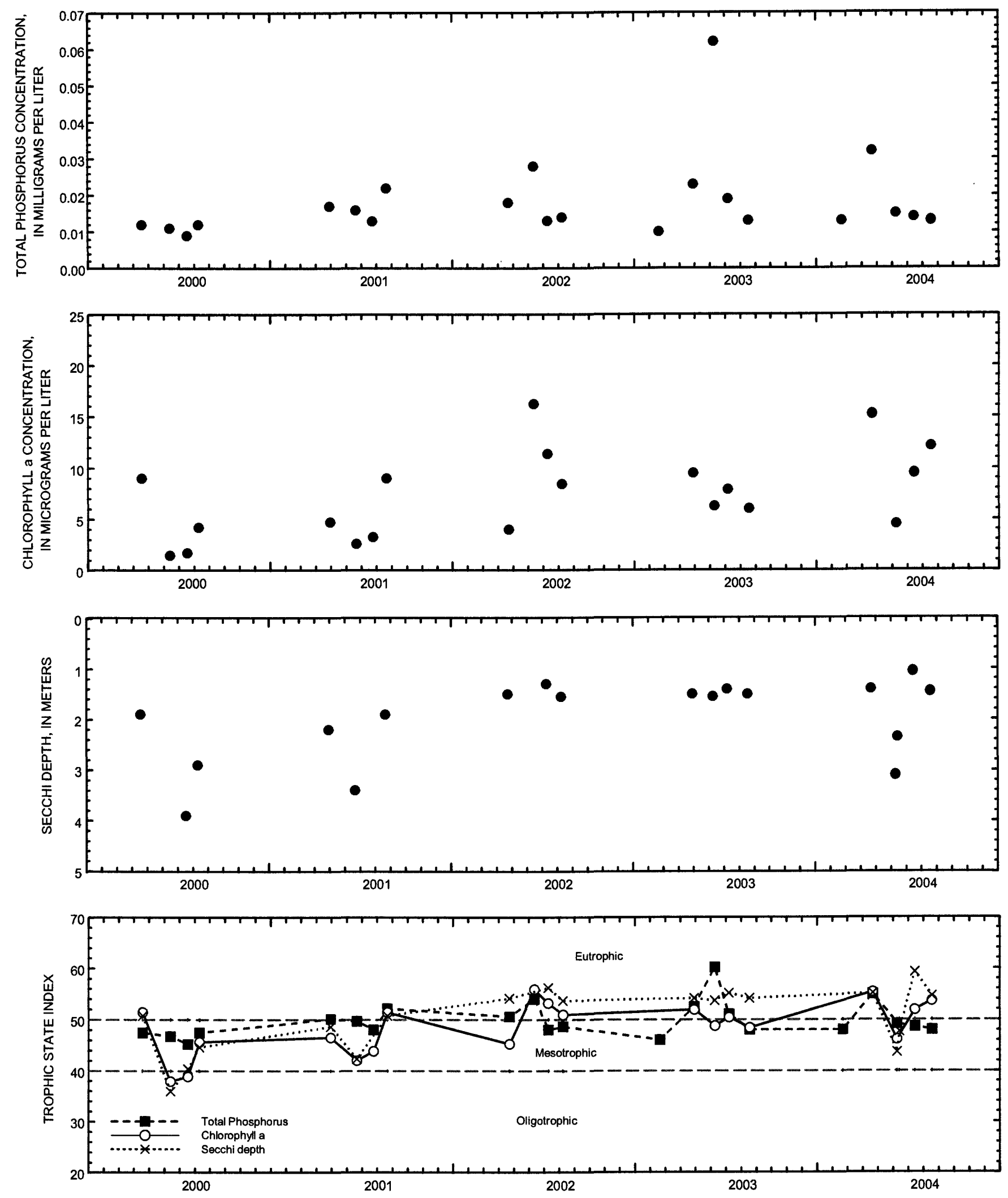

Surface total phosphorus, chlorophyll a concentrations, Secchi depths, and TSI data for Big Cedar Lake, North Site, near West Bend, Wisconsin. 
LOCATION.--Lat $43^{\circ} 22^{\prime} 24^{\prime \prime}$, long $88^{\circ} 15^{\prime} 49^{\prime \prime}$, in NE 1/4 SE 1/4 sec.31, T.11 N., R.19 E., Washington County, Hydrologic Unit 04040003, near West Bend.

PERIOD OF RECORD,--February 2000 to current year.

REMARKS.--Lake sampled on south side at deep hole. Lake ice-covered during February sampling. Water-quality analyses by Wisconsin State Laboratory of Hygiene.

WATER-QUALITY DATA, FEBRUARY 24 TO AUGUST 18, 2004

(Milligrams per liter unless otherwise indicated)

Date

Secchi depth $(\mathrm{m})$

Depth of sample $(\mathrm{m})$

Chlorophyll a, phytopiankton ( $\mu \mathrm{g} / \mathrm{L})$

Water temperature $\left({ }^{\circ} \mathrm{C}\right)$

Specific conductance $(\mu \mathrm{S} / \mathrm{cm})$

$\mathrm{pH}$

Dissolved oxygen (mg/L)

Phosphorus, total (as P)

Phosphorus, ortho, dissolved (as $\mathrm{P}$ )

Nitrogen, $\mathrm{NO}_{2}+\mathrm{NO}_{3}$, diss. (as $\mathrm{N}$ )

Nitrogen, ammonia, dissolved (as $\mathrm{N}$ )

Nitrogen, amm. + diss., total (as N)

Nitrogen, amm. + org., total (as $\mathrm{N}$ )

Nitrogen, total (as N)

Color (Pt-Co. scale)

Turbidity (NTU)

Hardness, as $\mathrm{CaCO}_{3}$

Calcium, dissolved (Ca)

Magnesium, dissolved (Mg)

Sodium, dissolved $(\mathrm{Na})$

Potassium, dissolved (K)

Alkalinity, as $\mathrm{CaCO}_{3}$

Sulfate, dissolved $\left(\mathrm{SO}_{4}\right)$

Chloride, dissolved (Cl)

Silica, dissolved $\left(\mathrm{SiO}_{2}\right)$

Solids, dissolved, at $180^{\circ} \mathrm{C}$

Iron, dissolved $(\mathrm{Fe})(\mu \mathrm{g} / \mathrm{L}$ )

Manganese, dissolved, $(\mathrm{Mn})(\mu \mathrm{g} / \mathrm{L})$

\begin{tabular}{|c|c|c|c|c|c|c|c|c|c|}
\hline \multicolumn{2}{|c|}{ Feb-24 } & \multicolumn{2}{|c|}{ Apr-22 } & \multicolumn{2}{|c|}{ Jun-9 } & \multicolumn{2}{|c|}{ Jul-14 } & \multicolumn{2}{|c|}{ Aug-18 } \\
\hline 0.5 & 30 & 0.5 & 30 & 0.5 & 30 & 0.5 & 30.5 & 0.5 & 30 \\
\hline- & - & 8.8 & - & 1.49 & - & 7.6 & - & 8.4 & - \\
\hline 0.9 & 2.6 & 8.3 & 6.1 & 21.8 & 7.5 & 23.2 & 7.7 & 20.8 & 7.5 \\
\hline 570 & 596 & 549 & 550 & 519 & 541 & 500 & 545 & 498 & 563 \\
\hline 7.9 & 7.4 & 8.3 & 8.1 & 8.3 & 7.5 & 8.4 & 7.5 & 8.5 & 7.4 \\
\hline 13.5 & 1.1 & 12.1 & 11.4 & 11.1 & 1.2 & 13.3 & 0.4 & 12.2 & 0.3 \\
\hline 0.017 & 0.083 & 0.017 & 0.019 & 0.010 & 0.061 & 0.016 & 0.202 & 0.012 & 0.253 \\
\hline- & - & 0.002 & -- & - & - & 0.003 & - & - & -- \\
\hline- & - & 0.124 & - & - & -- & $<0.019$ & - & -- & - \\
\hline- & - & 0.072 & - & - & - & $<0.015$ & - & - & - \\
\hline - & - & -- & - & - & - & 0.62 & - & - & -- \\
\hline- & - & 0.49 & - & - & -- & -- & - & - & - \\
\hline- & - & 0.61 & - & -- & -- & - & - & - & -- \\
\hline- & - & 5 & - & -- & - & - & - & - & - \\
\hline - & - & 1.9 & - & -- & - & - & - & - & - \\
\hline- & - & 230 & - & - & - & -- & - & $\rightarrow$ & - \\
\hline- & - & 36 & - & - & - & - & - & - & - \\
\hline- & - & 33.7 & - & - & - & - & - & - & -- \\
\hline- & - & 20.4 & - & - & - & - & - & - & - \\
\hline- & - & 2 & - & -- & -- & - & - & - & -- \\
\hline- & - & 192 & - & -- & - & - & - & $=$ & - \\
\hline- & - & 20.5 & - & -- & -- & - & - & - & - \\
\hline- & - & 44.3 & -- & -- & - & - & - & - & -- \\
\hline- & - & 0.389 & - & - & - & - & - & - & - \\
\hline- & - & 306 & -- & -- & - & -- & - & - & -- \\
\hline- & - & $<100$ & - & -- & - & - & - & - & - \\
\hline . & - & $<1$ & - & - & - & - & - & - & - \\
\hline
\end{tabular}

2-24-04

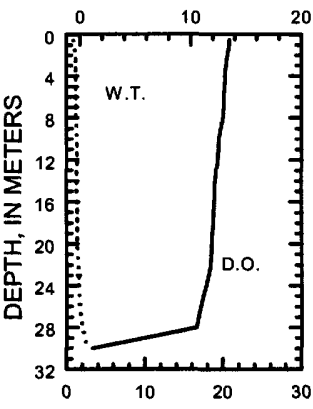

4-22-04

6-9-04

7-14-04

8-18-04

DISSOLVED OXYGEN (D.O.) IN MILLIGRAMS PER LITER
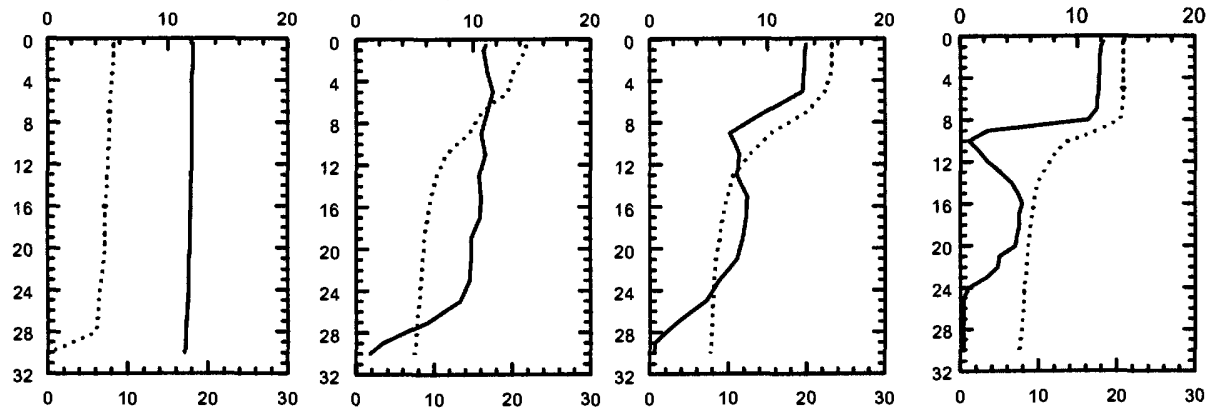

WATER TEMPERATURE (W.T.) IN DEGREES CELSIUS

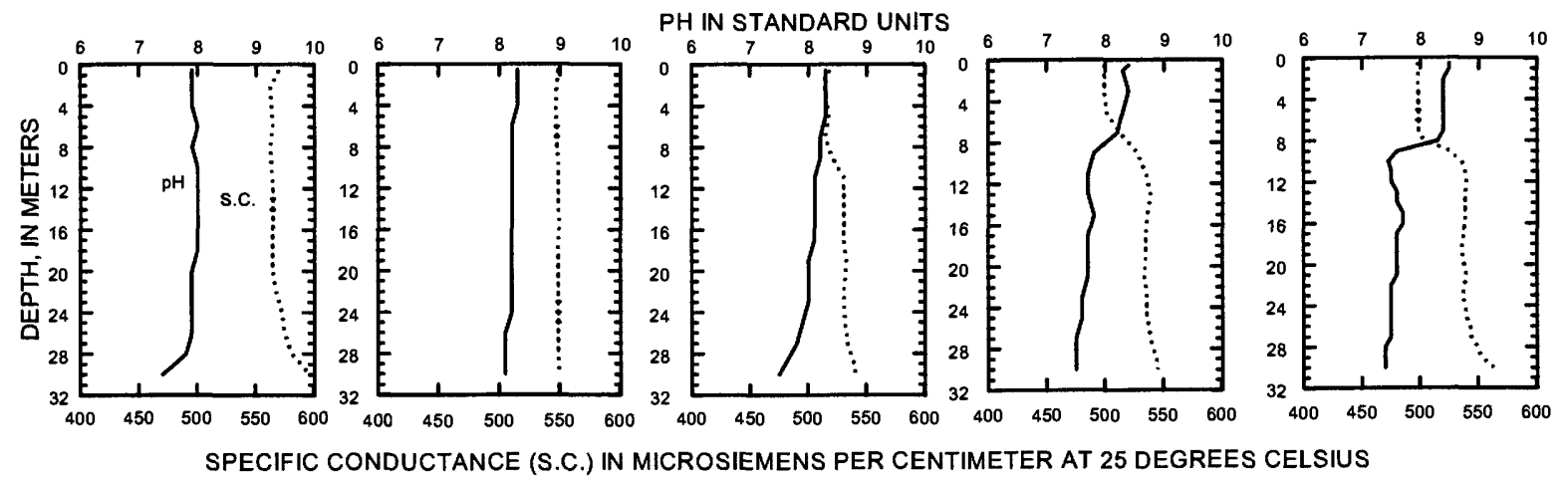



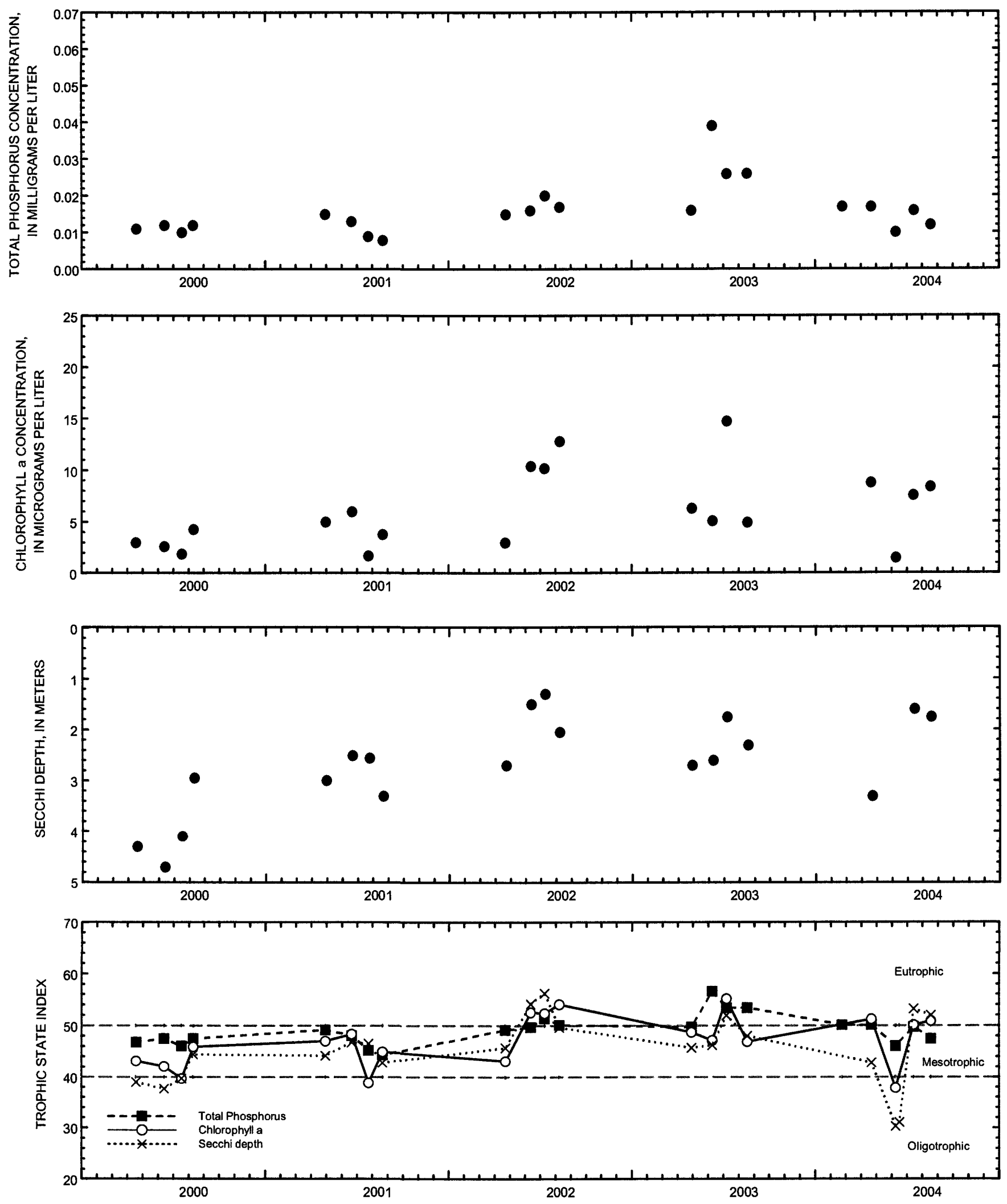

Surface total phosphorus, chlorophyll a concentrations, Secchi depths, and TSI data for Big Cedar Lake, South Site, near West Bend, Wisconsin. 


\section{BUTTERNUT LAKE NEAR PARK FALLS, WI}

LOCATION.--Lat 4558'54", long 90³1'03", in NW 1/4 NW 1/4 NE 1/4 sec.5, T.40 N., R.1 W., Ashland County, Hydrologic Unit 07050002, about $150 \mathrm{ft}$ south of Wisconsin Department of Natural Resources boat landing off County Highway B about 5 mi northwest of Park Falls.

DRAINAGE AREA.--47.6 $\mathrm{mi}^{2}$, at lake outlet. Area of Butternut Lake is $1.57 \mathrm{mi}^{2}$.

PERIOD OF RECORD.--October 2002 to October 2004.

GAGE.--Water-stage recorder. Datum of gage is about $1,495 \mathrm{ft}$ above sea level.

EXTREMES FOR PERIOD OF RECORD (NOVEMBER 2002 TO OCTOBER 2004).--Maximum recorded gage height, $7.38 \mathrm{ft}$, May 13, 2003 ; minimum recorded, $4.79 \mathrm{ft}$, July 30, 2003.

EXTREMES FOR CURRENT PERIOD (OCTOBER 2003 TO OCTOBER 2004).--Maximum recorded gage height, $7.17 \mathrm{ft}$, April 21 ; minimum recorded, $4.88 \mathrm{ft}$, July 29 .

WATER YEAR OCTOBER 2003 TO OCTOBER 2004

DAILY MEAN VALUES

\begin{tabular}{|c|c|c|c|c|c|c|c|c|c|c|c|c|c|}
\hline DAY & OCT & NOV & DEC & JAN & FEB & MAR & APR & MAY & JUN & JUL & AUG & SEP & OCT \\
\hline 1 & 5.12 & 5.27 & 5.12 & 5.03 & 4.95 & 4.99 & 6.06 & 5.49 & 5.71 & 5.01 & 5.01 & 5.11 & 5.01 \\
\hline 2 & 5.12 & 5.27 & 5.10 & 5.03 & 4.99 & 5.01 & 6.06 & 5.42 & 5.76 & 5.00 & 5.01 & 5.12 & 5.06 \\
\hline 3 & 5.12 & 5.26 & 5.08 & 5.04 & 5.01 & 5.02 & 6.07 & 5.37 & 5.72 & 4.99 & 5.00 & 5.12 & 5.07 \\
\hline 4 & 5.12 & 5.27 & 5.07 & 5.03 & 5.00 & 5.01 & 6.04 & 5.31 & 5.65 & 5.04 & 4.96 & 5.13 & 5.07 \\
\hline 5 & 5.12 & 5.26 & 5.06 & 5.03 & 4.99 & 5.04 & 6.00 & 5.28 & 5.60 & 5.06 & 4.95 & 5.24 & 5.08 \\
\hline 6 & 5.12 & 5.25 & 5.05 & 5.02 & 5.00 & 5.05 & 5.99 & 5.22 & 5.57 & 5.06 & 4.93 & 5.43 & 5.09 \\
\hline 7 & 5.11 & 5.23 & 5.04 & 5.01 & 5.00 & 5.07 & 5.99 & 5.19 & 5.54 & 5.08 & 4.93 & 5.44 & 5.10 \\
\hline 8 & 5.12 & 5.21 & 5.04 & 5.00 & 4.99 & 5.06 & 6.03 & 5.16 & 5.51 & 5.07 & 4.96 & 5.44 & 5.13 \\
\hline 9 & 5.13 & 5.19 & 5.06 & 4.99 & 4.99 & 5.05 & 6.04 & 5.16 & 5.49 & 5.06 & 4.99 & 5.40 & 5.13 \\
\hline 10 & 5.13 & 5.17 & 5.08 & 4.98 & 4.99 & 5.05 & 6.04 & 5.18 & 5.45 & 5.05 & 5.03 & 5.36 & 5.13 \\
\hline 11 & 5.16 & 5.16 & 5.07 & 4.97 & 4.99 & 5.07 & 5.99 & 5.17 & 5.40 & 5.05 & 5.06 & 5.31 & 5.13 \\
\hline 12 & 5.21 & 5.17 & 5.06 & 4.97 & 4.99 & 5.06 & 5.90 & 5.18 & 5.39 & 5.06 & 5.08 & 5.26 & 5.14 \\
\hline 13 & 5.21 & 5.16 & 5.05 & 4.97 & 4.99 & 5.07 & 5.80 & 5.23 & 5.37 & 5.06 & 5.10 & 5.22 & 5.14 \\
\hline 14 & 5.22 & 5.15 & 5.04 & 4.99 & 4.99 & 5.08 & 5.70 & 5.38 & 5.33 & 5.05 & 5.10 & 5.17 & 5.15 \\
\hline 15 & 5.22 & 5.15 & 5.03 & 4.99 & 4.97 & 5.06 & 5.62 & 5.47 & 5.30 & 5.04 & 5.10 & 5.24 & 5.15 \\
\hline 16 & 5.21 & 5.16 & 5.04 & 4.99 & 4.96 & 5.05 & 5.57 & 5.50 & 5.26 & 5.01 & 5.11 & 5.26 & 5.16 \\
\hline 17 & 5.20 & 5.19 & 5.03 & 5.00 & 4.95 & 5.05 & 5.54 & 5.48 & 5.23 & 4.98 & 5.12 & 5.26 & 5.16 \\
\hline 18 & 5.20 & 5.29 & 5.03 & 4.99 & 4.95 & 5.04 & 5.64 & 5.44 & 5.20 & 4.97 & 5.12 & 5.25 & 5.16 \\
\hline 19 & 5.20 & 5.34 & 5.02 & 4.98 & 4.95 & 5.03 & 6.25 & 5.40 & 5.17 & 4.96 & 5.09 & 5.23 & 5.16 \\
\hline 20 & 5.20 & 5.37 & 5.02 & 4.98 & 4.99 & 5.03 & 6.96 & 5.35 & 5.14 & 5.00 & 5.07 & 5.20 & 5.17 \\
\hline 21 & 5.19 & 5.36 & 5.01 & 4.99 & 4.99 & 5.01 & 7.11 & 5.31 & 5.11 & 5.03 & 5.06 & 5.16 & 5.18 \\
\hline 22 & 5.18 & 5.33 & 5.00 & 4.98 & 4.98 & 5.00 & 6.89 & 5.28 & 5.09 & 5.00 & 5.05 & 5.13 & 5.20 \\
\hline 23 & 5.16 & 5.32 & 5.00 & 4.98 & 4.99 & 4.99 & 6.60 & 5.29 & 5.09 & 4.97 & 5.05 & 5.11 & 5.28 \\
\hline 24 & 5.17 & 5.29 & 5.00 & 4.98 & 4.99 & 4.99 & 6.32 & 5.37 & 5.09 & 4.97 & 5.05 & 5.10 & 5.34 \\
\hline 25 & 5.18 & 5.27 & 4.99 & 4.98 & 4.98 & 5.00 & 6.13 & 5.44 & 5.09 & 4.95 & 5.06 & 5.08 & 5.37 \\
\hline 26 & 5.18 & 5.23 & 4.99 & 4.97 & 4.98 & 5.04 & 5.98 & 5.47 & 5.07 & 4.93 & 5.07 & 5.06 & 5.38 \\
\hline 27 & 5.18 & 5.20 & 4.99 & 4.98 & 4.97 & 5.11 & 5.86 & 5.49 & 5.07 & 4.92 & 5.08 & 5.04 & 5.39 \\
\hline 28 & 5.21 & 5.18 & 5.01 & 4.97 & 4.97 & 5.31 & 5.76 & 5.47 & 5.05 & 4.91 & 5.08 & 5.02 & 5.42 \\
\hline 29 & 5.22 & 5.16 & 5.02 & 4.96 & 4.97 & 5.65 & 5.66 & 5.45 & 5.04 & 4.94 & 5.08 & 5.00 & 5.50 \\
\hline 30 & 5.25 & 5.14 & 5.03 & 4.96 & --- & 5.89 & 5.58 & 5.46 & 5.03 & 4.95 & 5.10 & 4.99 & 5.58 \\
\hline 31 & 5.27 & -- & 5.04 & 4.95 & --- & 6.01 & - & 5.59 & -- & 5.00 & 5.10 & --- & 5.61 \\
\hline MEAN & 5.18 & 5.23 & 5.04 & 4.99 & 4.98 & 5.13 & 6.04 & 5.35 & 5.32 & 5.01 & 5.05 & 5.20 & 5.21 \\
\hline MAX & 5.27 & 5.37 & 5.12 & 5.04 & 5.01 & 6.01 & 7.11 & 5.59 & 5.76 & 5.08 & 5.12 & 5.44 & 5.61 \\
\hline MIN & 5.11 & 5.14 & 4.99 & 4.95 & 4.95 & 4.99 & 5.54 & 5.16 & 5.03 & 4.91 & 4.93 & 4.99 & 5.01 \\
\hline
\end{tabular}




\section{BUTTERNUT LAKE, DEEP HOLE, NEAR PARK FALLS, WI}

LOCATION.--Lat $45^{\circ} 58^{\prime} 03^{\prime \prime}$, long $90^{\circ} 31^{\prime} 08^{\prime \prime}$, in NW 1/4 NW 1/4 NE 1/4 sec.8, T.40 N., R.1 W., Ashland County, Hydrologic Unit 07050002, near

Park Falls.

PERIOD OF RECORD.--March 2003 to current year.

REMARKS.--Lake sampled at deep hole at a lake depth of about $8 \mathrm{~m}$. Lake ice-covered during March sampling. Water-quality analyses done by Wisconsin State Laboratory of Hygiene.

WATER-QUALITY DATA, MARCH 17 TO JUNE 2, 2004

(Milligrams per liter unless otherwise indicated)

Date

Lake stage (ft)

Secchi depth $(m)$

Depth of sample $(\mathrm{m})$

Chlorophyll a, phytoplankton ( $\mu \mathrm{g} / \mathrm{L}$ )

Water temperature $\left({ }^{\circ} \mathrm{C}\right)$

Specific conductance $(\mu \mathrm{S} / \mathrm{cm})$

$\mathrm{pH}$

Dissolved oxygen (mg/L)

Phosphorus, total (as P)

Phosphorus, ortho, dissolved (as $\mathrm{P}$ )

Nitrogen, $\mathrm{NO}_{2}+\mathrm{NO}_{3}$, diss. (as $\mathrm{N}$ )

Nitrogen, ammonia, dissolved (as $\mathrm{N}$ )

Nitrogen, amm. + org., total (as $\mathrm{N}$ )

Nitrogen, total (as N)

Color (Pt-Co. scale)

Turbidity (NTU)

Hardness, as $\mathrm{CaCO}_{3}$

Calcium, dissolved $(\mathrm{Ca})$

Magnesium, dissolved (Mg)

Sodium, dissolved $(\mathrm{Na})$

Potassium, dissolved (K)

Alkalinity, as $\mathrm{CaCO}_{3}$

Sulfate, dissolved $\left(\mathrm{SO}_{4}\right)$

Chloride, dissolved (Cl)

Silica, dissolved $\left(\mathrm{SiO}_{2}\right)$

Solids, dissolved, at $180^{\circ} \mathrm{C}$

iron, dissolved ( $\mathrm{Fe})(\mu \mathrm{g} / \mathrm{L}$ )

Manganese, dissolved, (Mn) ( $\mu \mathrm{g} / \mathrm{L})$

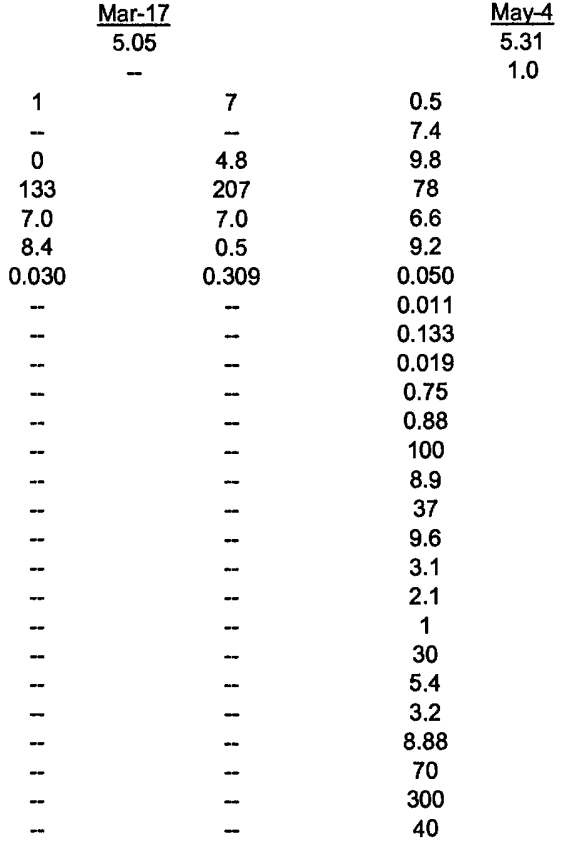

5-4-04

DISSOLVED OXYGEN (D.O.) IN MILLIGRAMS PER LITER
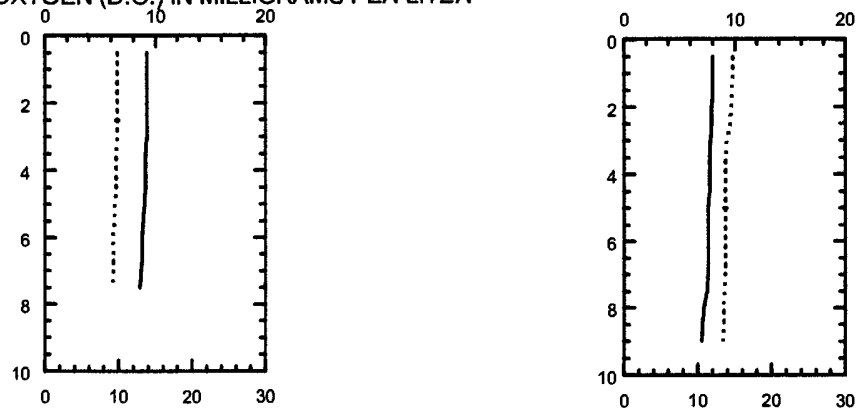

WATER TEMPERATURE (W.T.) IN DEGREES CELSIUS

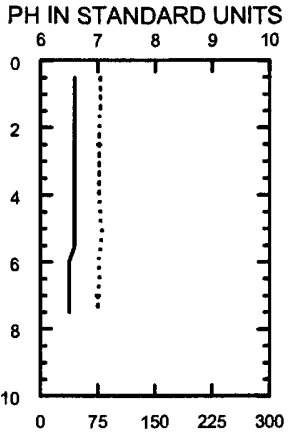

7.5
-
9.8
75
6.5
8.6
0.044
-
--
-
-
-
-
-
-
-
-
-
-
-
-
-
--
-
-
--
-
--
-
-

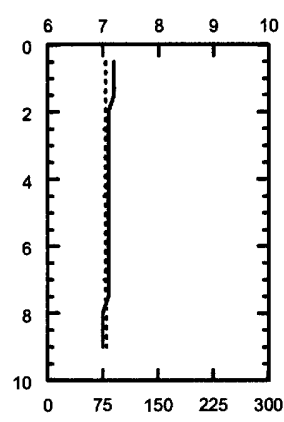

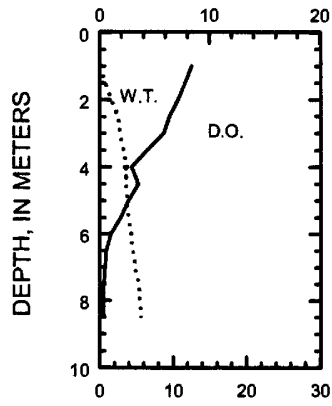

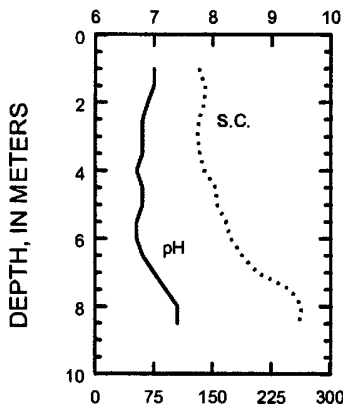

SPECIFIC CONDUCTANCE (S.C.) IN MICROSIEMENS PER CENTIMETER AT 25 DEGREES CELSIUS 
WATER-QUALITY DATA, JULY 21 TO SEPTEMBER 21, 2004

(Milligrams per leiter unless otherwise indicated)

Date

Lake stage (ft)

Secchi depth $(\mathrm{m})$

Depth of sample $(\mathrm{m})$

Chlorophyll a, phytoplankton $(\mu \mathrm{g} / \mathrm{L})$

Water temperature $\left({ }^{\circ} \mathrm{C}\right)$

Specific conductance $(\mu \mathrm{S} / \mathrm{cm})$

$\mathrm{pH}$

Dissolved oxygen (mg/L)

Phosphorus, total (as P)

Phosphorus, ortho, dissolved (as $\mathrm{P}$ )

Nitrogen, $\mathrm{NO}_{2}+\mathrm{NO}_{3}$, diss. (as $\mathrm{N}$ )

Nitrogen, ammonia, dissolved (as $\mathrm{N}$ )

Nitrogen, amm. + diss., total (as N)

\begin{tabular}{cccccc} 
& $\frac{\mathrm{Jul}-21}{5.03}$ & & \multicolumn{3}{c}{$\frac{\text { Aug-10 }}{5.03}$} \\
& 0.9 & & \multicolumn{2}{c}{1.0} & \\
0.5 & & 9 & 0.5 & 8 \\
35.7 & & - & 29.2 & -- \\
25.0 & & 18.3 & 20.6 & 18.8 \\
91 & & 100 & 102.5 & 142.6 \\
8.6 & & 6.5 & 7.1 & 7.0 \\
10.9 & 0.6 & 7.3 & 0.1 \\
0.044 & & 0.059 & 0.054 & 0.351 \\
0.002 & -- & - & - \\
0.019 & & -- & - & - \\
0.025 & -- & - & - \\
0.82 & -- & - & -
\end{tabular}

\section{$7-21-04$}
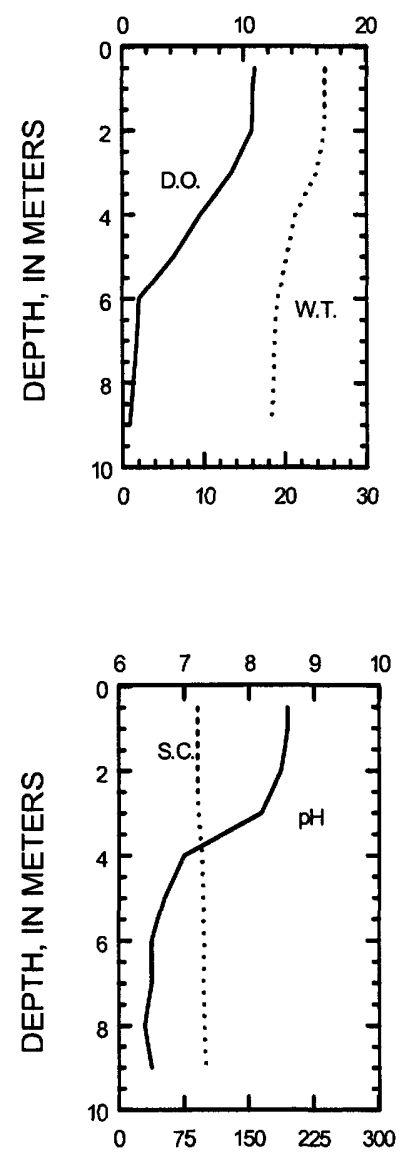

8-10-04

DISSOLVED OXYGEN (D.O.) IN MLLLIGRAMS PER LITER

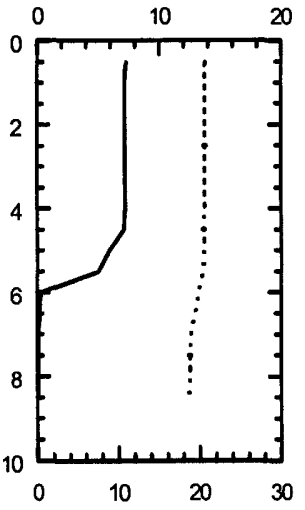

WATER TEMPERATURE (W.T.) IN DEGREES CELSIUS

PH IN STANDARD UNITS

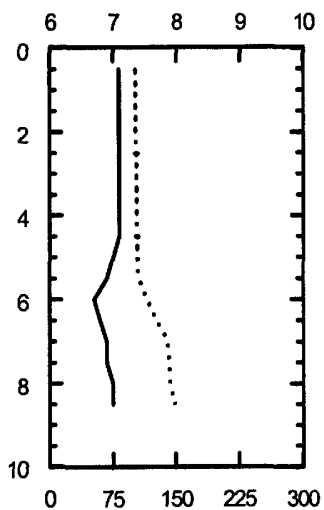

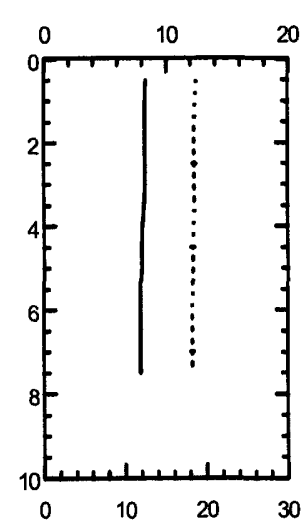

9-21-04

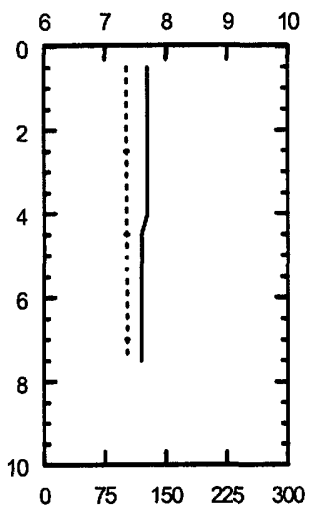

SPECIFIC CONDUCTANCE (S.C.) IN MICROSIEMENS PER CENTIMETERAT 25 DEGREES CELSIUS 

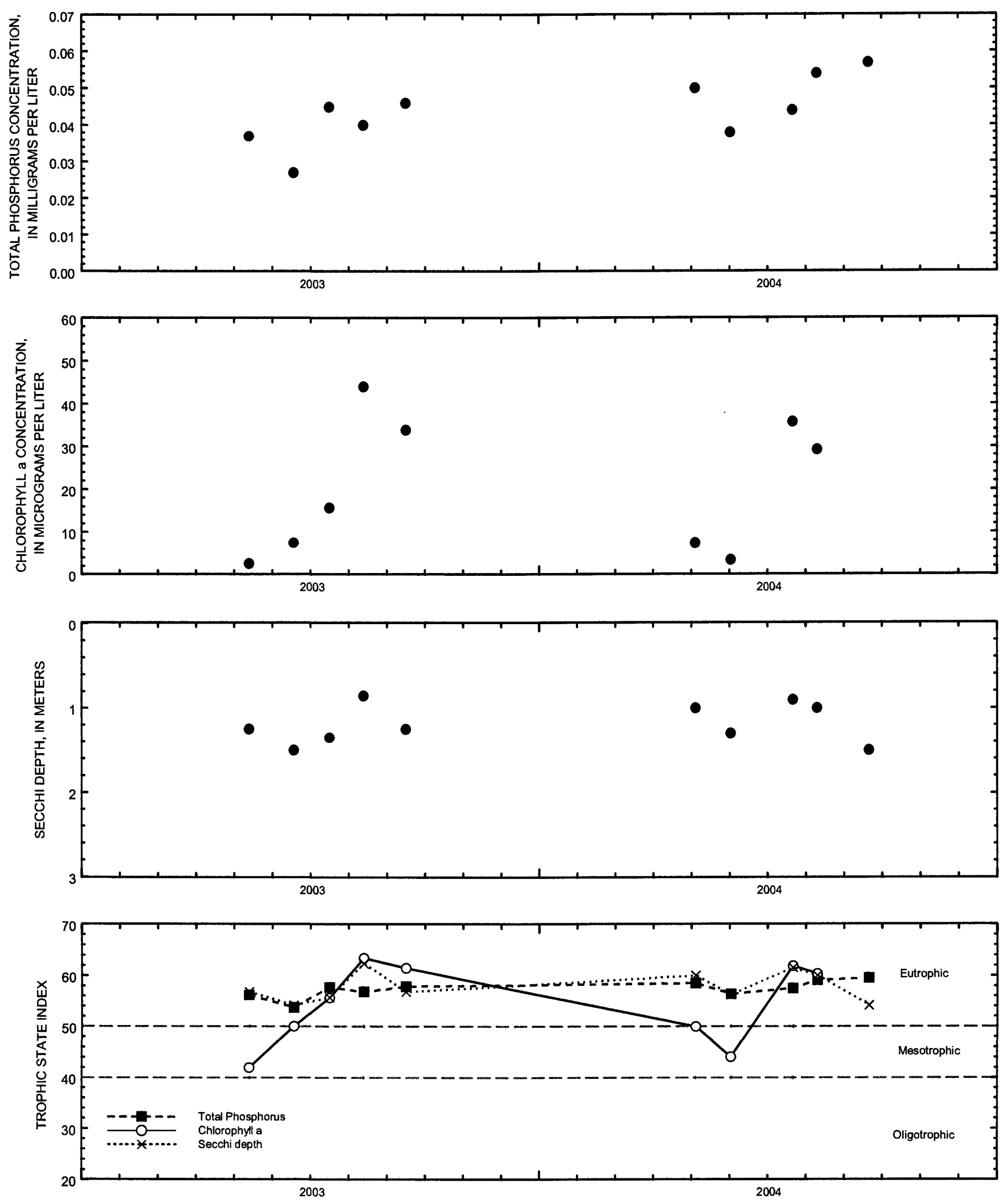

Surface total phosphorus, chlorophyll a concentrations, Secchi depths, and TSI data for Butternut Lake, Deep Hole, near Park Falls, Wisconsin. 


\section{BUTTERNUT LAKE, NORTH SITE, NEAR BUTTERNUT, WI}

LOCATION.--Lat 45 59'04", long 90³0'34", in NE 1/4 SE 1/4 NE 1/4 sec.32, T.41 N., R.1 W., Ashland County, Hydrologic Unit 07050002, near Butternut.

PERIOD OF RECORD.--March 2004 to current year.

REMARKS.--Lake sampled at North Site at a lake depth of about $5.5 \mathrm{~m}$. Lake ice-covered during March sampling. Water-quality analyses done by Wisconsin State Laboratory of Hygiene.

WATER-QUALITY DATA, MARCH 17 TO JUNE 2, 2004

(Milligrams per liter unless otherwise indicated)

Date

Lake stage (ft)

Secchi depth $(\mathrm{m})$

Depth of sample $(\mathrm{m})$

Chiorophyll a, phytoplankton $(\mu \mathrm{g} / \mathrm{L})$

Water temperature $\left({ }^{\circ} \mathrm{C}\right)$

Specific conductance $(\mu \mathrm{S} / \mathrm{cm})$

$\mathrm{pH}$

Dissolved oxygen (mg/L)

Phosphorus, total (as P)
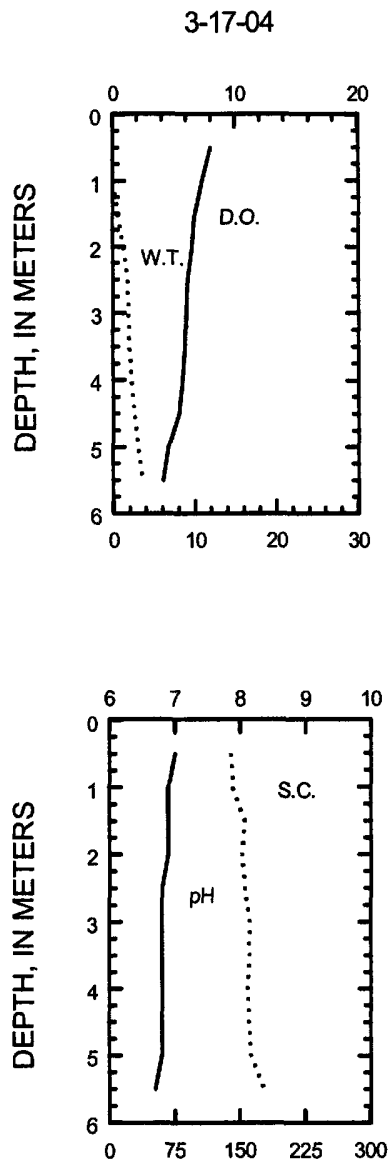

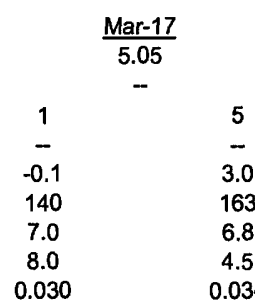

$\frac{\text { May }-4}{5.31}$

$\begin{array}{cc}0.5 & 5.5 \\ 4.1 & - \\ 9.6 & 9.4 \\ 66 & 67 \\ 6.4 & 6.4 \\ 8.7 & 8.5 \\ 0.046 & 0.040\end{array}$

5-4-04

DISSOLVED OXYGEN (D.O.) IN MILLIGRAMS PER LITER
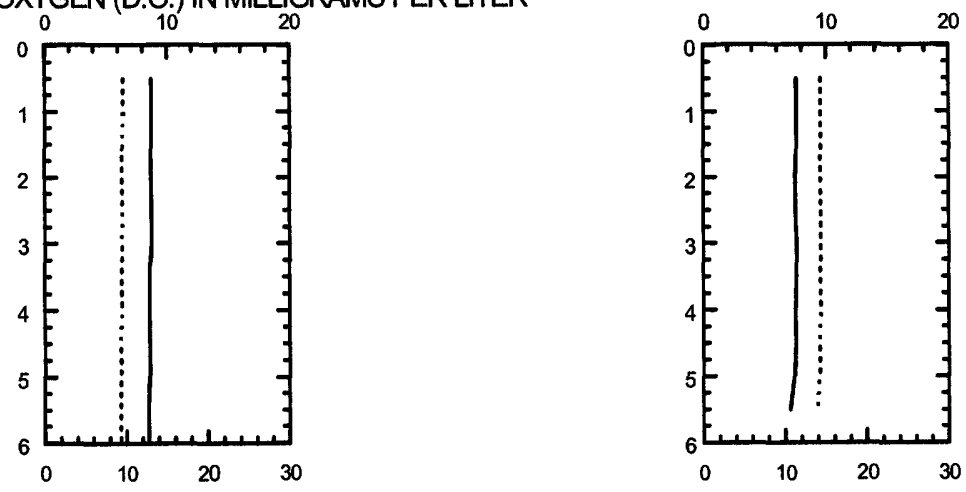

WATER TEMPERATURE (W.T.) IN DEGREES CELSIUS

PH IN STANDARD UNITS
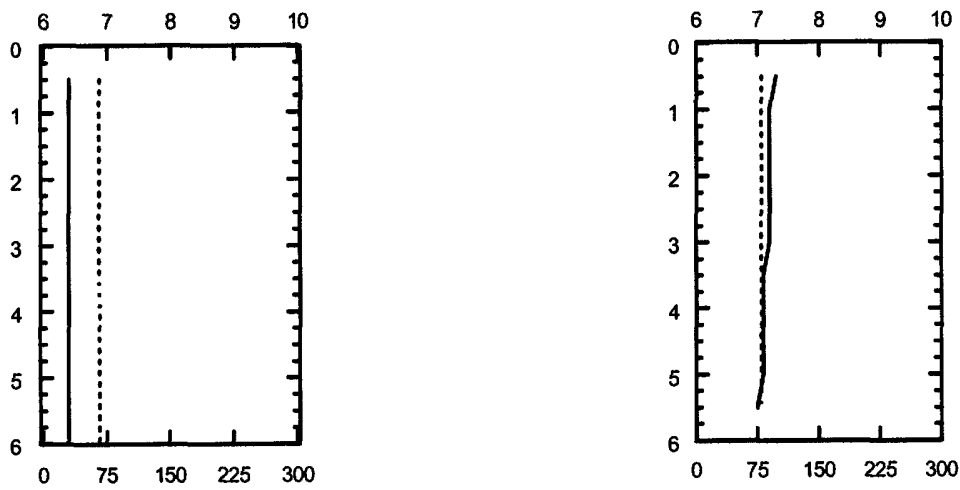

SPECIFIC CONDUCTANCE (S.C.) IN MICROSIEMENS PER CENTIMETER AT 25 DEGREES CELSIUS 
WATER-QUALITY DATA, JULY 21 TO SEPTEMBER 21, 2004

(Milligrams per leiter unless otherwise indicated)

Date

Lake stage (ft)

Secchi depth $(m)$

Depth of sample $(\mathrm{m})$

Chlorophyll a, phytoplankton $(\mu \mathrm{g} / \mathrm{L})$

Water temperature $\left({ }^{\circ} \mathrm{C}\right)$

Specific conductance $(\mu \mathrm{S} / \mathrm{cm})$

$\mathrm{pH}$

Dissolved oxygen (mg/L)

Phosphorus, total (as P)

$\begin{array}{ccc} & \frac{J u l-21}{5.03} & \\ & 1.0 & \\ & & \\ 0.5 & & 5.8 \\ 34.6 & & - \\ 25.2 & & 17.9 \\ 94 & & 110 \\ 8.7 & & 6.6 \\ 11.3 & & 0.2 \\ 0.045 & & 0.042\end{array}$

7-21-04
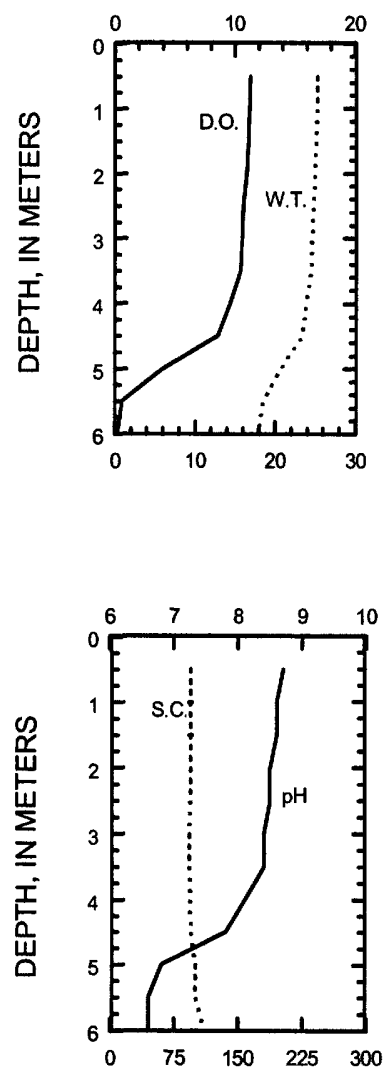

8-10-04

DISSOLVED OXYGEN (D.O.) IN MILLIGRAMS PER LITER

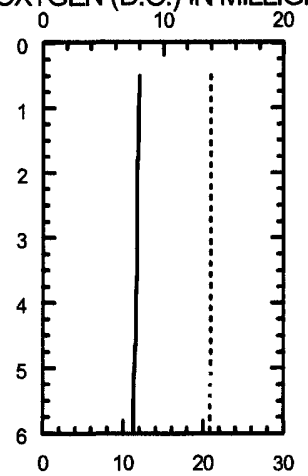

WATER TEMPERATURE (W.T.) IN DEGREES CELSIUS

PH IN STANDARD UNITS

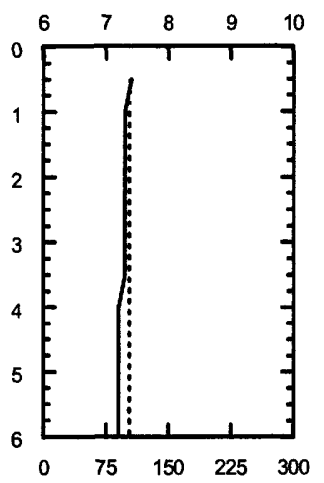

$\begin{array}{cc}\frac{\text { Aug-10 }}{5.03} & \\ 1.0 & \\ & 5 \\ & -- \\ & 20.9 \\ & 103 \\ & 7.2 \\ & 7.6 \\ & 0.057\end{array}$

5.16

1.1

$\begin{array}{cc}0.5 & 5.5 \\ - & - \\ 18.9 & 18.5 \\ 104 & 104 \\ 7.7 & 7.6 \\ 8.4 & 8.0 \\ 0.067 & 0.063\end{array}$

SPECIFIC CONDUCTANCE (S.C.) IN MICROSIEMENS PER CENTIMETER AT 25 DEGREES CELSIUS

$9-21-04$
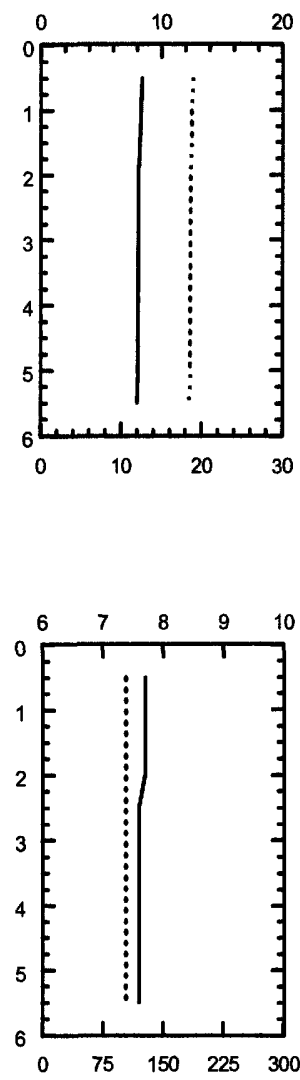

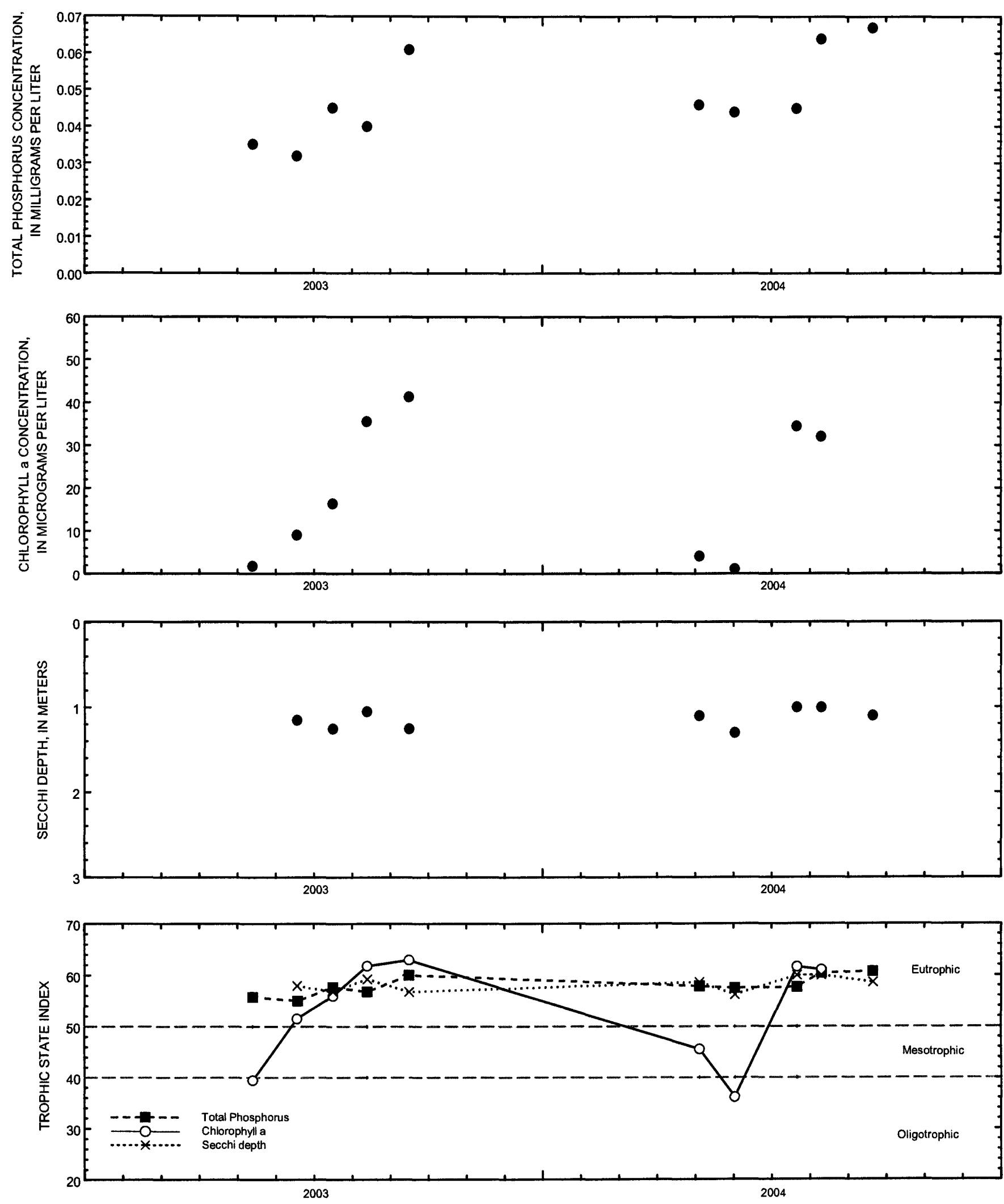

Surface total phosphorus, chlorophyll a concentrations, Secchi depths, and TSI data for Butternut Lake, North Site, near Butternut, Wisconsin. 
LOCATION.--Lat 4557'31", long 90³1'10", in SW 1/4 NW 1/4 SE 1/4 sec.8, T.40 N., R.1 W., Ashland County, Hydrologic Unit 07050002, near Park Falls.

PERIOD OF RECORD.--March 2003 to current year.

REMARKS.--Lake sampled at South Site at a lake depth of about $6 \mathrm{~m}$. Lake ice-covered during March sampling. Water-quality analyses done by Wisconsin State Laboratory of Hygiene.

WATER-QUALITY DATA, MARCH 17 TO JUNE 2, 2004

(Milligrams per liter unless otherwise indicated)

Date

Lake stage ( $\mathrm{ft}$ )

Secchi depth $(\mathrm{m})$

Depth of sample (m)

Chlorophyll a, phytoplankton ( $\mu \mathrm{g} / \mathrm{L})$

Water temperature $\left({ }^{\circ} \mathrm{C}\right)$

Specific conductance $(\mu \mathrm{S} / \mathrm{cm})$

$\mathrm{pH}$

Dissolved oxygen (mg/L)

Phosphorus, total (as P)

3-17-04
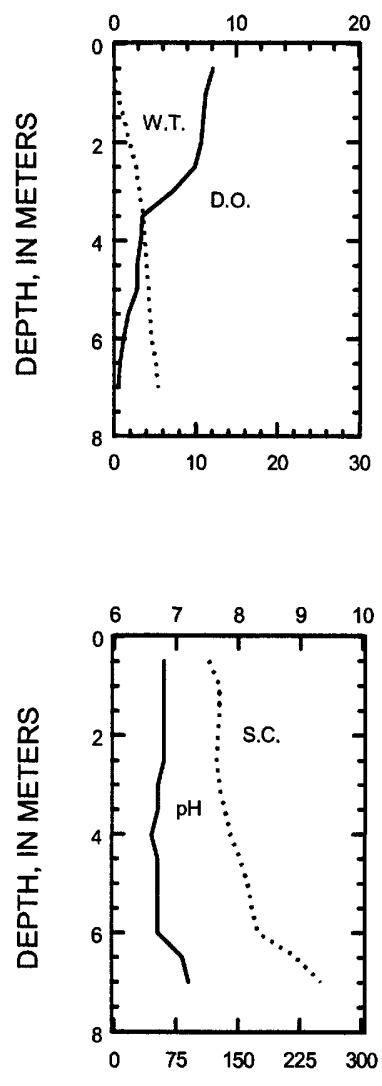

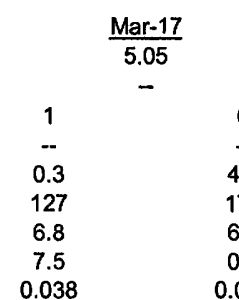

6
-
4.6
174
6.7
0.8
0.088

$\frac{\text { May- }-4}{5.31}$

1.2

$\begin{array}{cc}0.5 & 7 \\ 6.3 & -- \\ 9.4 & 9.1 \\ 80 & 81 \\ 6.7 & 6.5 \\ 9.1 & 8.3 \\ 0.047 & 0.048\end{array}$

5-4-04

DISSOLVED OXYGEN (D.O.) IN MILLIGRAMS PER LITER
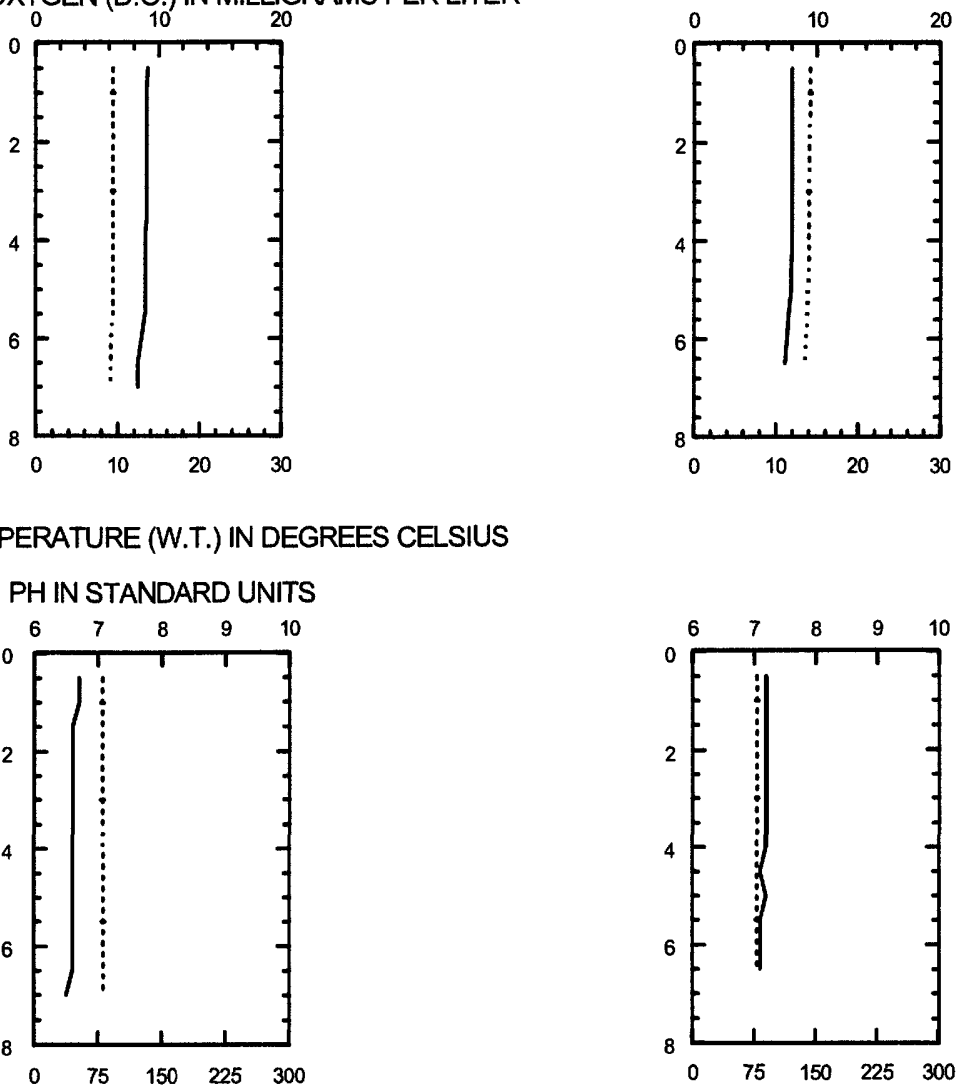

PH IN STANDARD UNITS

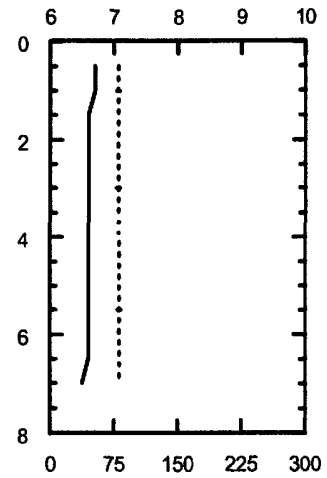

6-2-04

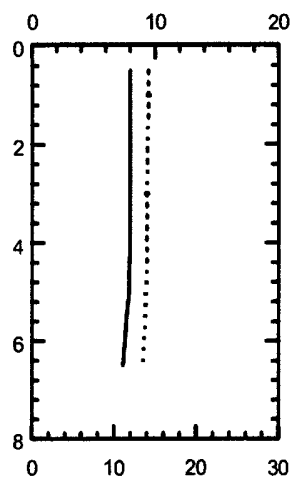

WATER TEMPERATURE (W.T.) IN DEGREES CELSIUS

SPECIFIC CONDUCTANCE (S.C.) IN MICROSIEMENS PER CENTIMETER AT 25 DEGREES CELSIUS 
WATER-QUALITY DATA, JULY 21 TO SEPTEMBER 21, 2004

(Milligrams per leiter unless otherwise indicated)

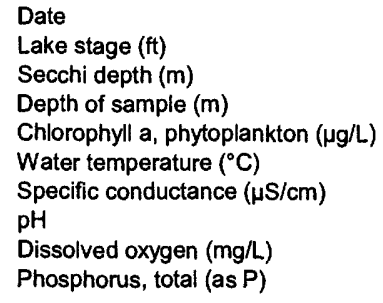

7-21-04
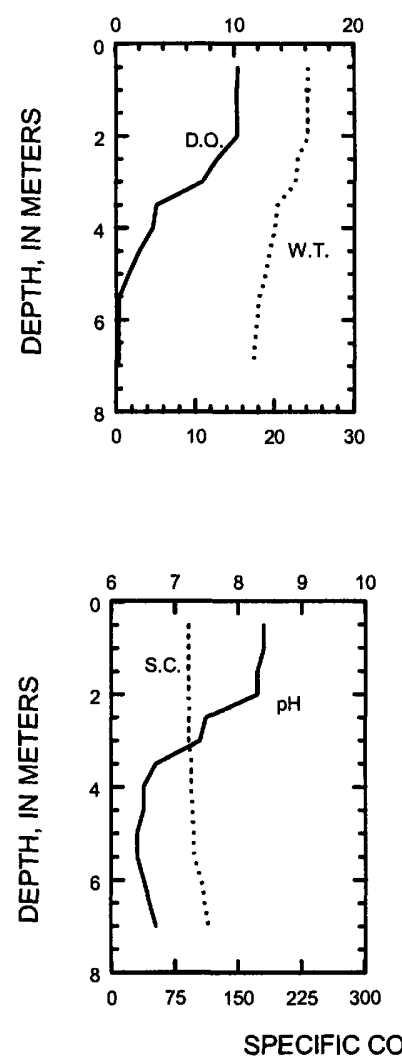

$\begin{array}{ccc} & \frac{\text { Jul-21 }}{} & \\ & 5.03 & \\ & 1.1 & \\ 0.5 & & 6.5 \\ 33.2 & & -- \\ 24.3 & & 17.5 \\ 91 & & 111 \\ 8.4 & & 6.6 \\ 10.3 & & 0.2 \\ 0.045 & & 0.10\end{array}$

8-10-04

DISSOLVED OXYGEN (D.O.) IN MILLIGRAMS PER LITER

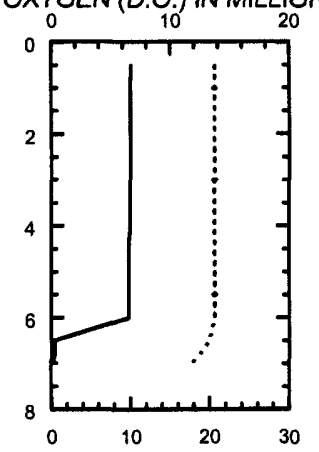

WATER TEMPERATURE (W.T.) IN DEGREES CELSIUS
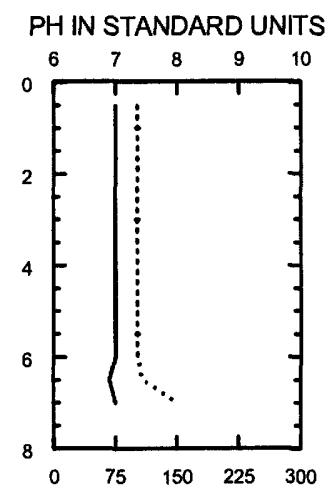

PH IN STANDARD UNITS

$\begin{array}{cc}\frac{\text { Aug-10 }}{5.03} & \\ 1.0 & \\ & 6 \\ & -- \\ & 20.6 \\ & 102.1 \\ & 7.0 \\ & 6.5 \\ & 0.050\end{array}$

$\frac{\text { Sep-21 }}{5.16}$

1.3

0.5
20.8

20.8
18.6

18.6
102

7.5

8.3
0.055
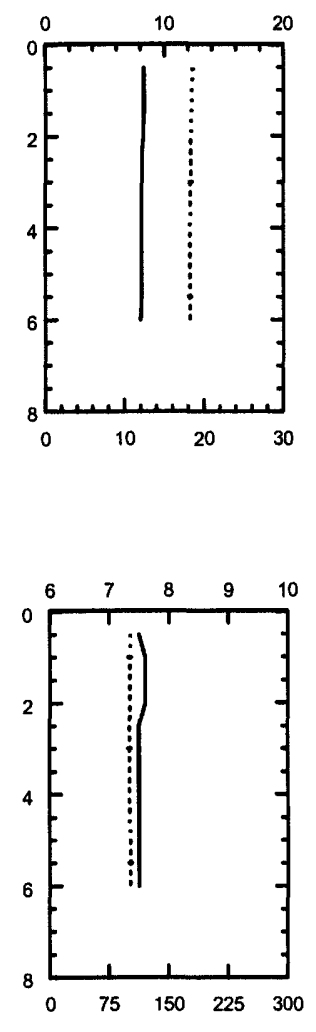

.) IN MICROSIEMENS PER CENTIMETER AT 25 DEGREES CELSIUS 

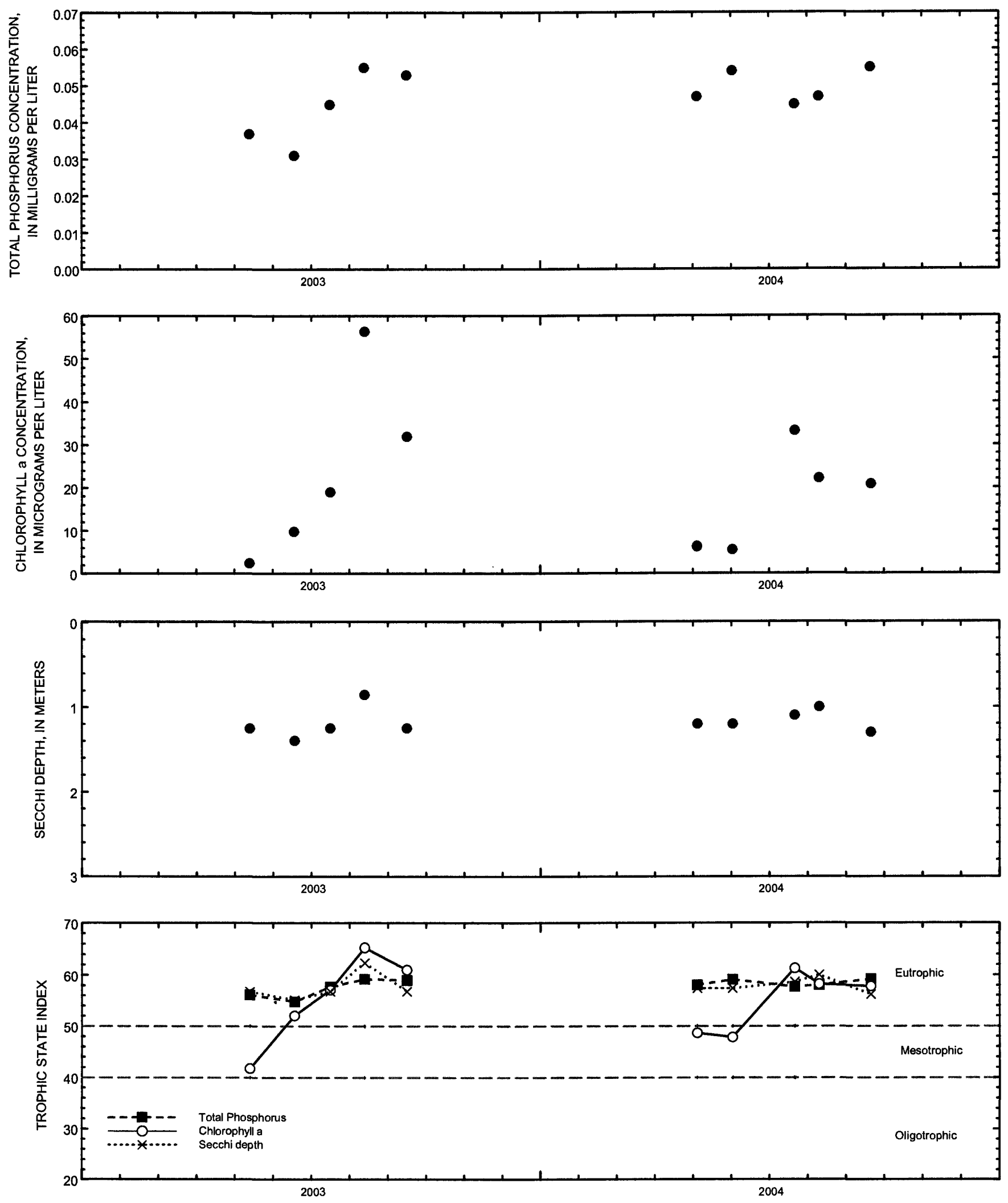

Surface total phosphorus, chlorophyll a concentrations, Secchi depths, and TSI data for Butternut Lake, South Site, near Park Falls, Wisconsin. 


\section{BUTTERNUT LAKE, FAR SOUTH SITE, NEAR PARK FALLS, WI}

LOCATION.--Lat 4556'51", long 90³1'27", in SW 1/4 SE 1/4 NE 1/4 sec.17, T.40 N., R.1 W., Ashland County, Hydrologic Unit 07050002, near Park Falls.

PERIOD OF RECORD.--March 2003 to current year.

REMARKS.--Lake sampled at deep hole at Far South Site at a lake depth of about $4 \mathrm{~m}$. Lake ice-covered during March sampling. Water-quality analyses done by Wisconsin State Laboratory of Hygiene.

WATER-QUALITY DATA, JUNE 2 TO SEPTEMBER 21, 2004

(Milligrams per liter unless otherwise indicated)

Date

Lake stage ( $\mathrm{ft}$ )

Secchi depth $(\mathrm{m})$

Depth of sample $(\mathrm{m})$

Water temperature $\left({ }^{\circ} \mathrm{C}\right.$

Specific conductance $(\mu \mathrm{S} / \mathrm{cm})$

$\mathrm{pH}$

Dissoived oxygen (mg/L)

\begin{tabular}{|c|c|}
\hline & $\begin{array}{c}\frac{\text { Jun-2 }}{5.76} \\
1.1\end{array}$ \\
\hline 0.5 & \\
\hline 14.7 & \\
\hline 79 & \\
\hline 7.1 & \\
\hline 8.4 & \\
\hline
\end{tabular}

7-21-04

DISSOLVEL

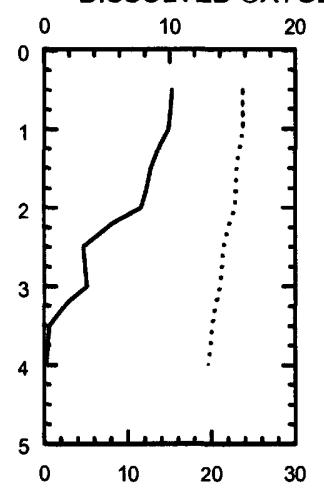

WATER TEMPERATURE (W.T.)

PH IN STANDARD UNITS
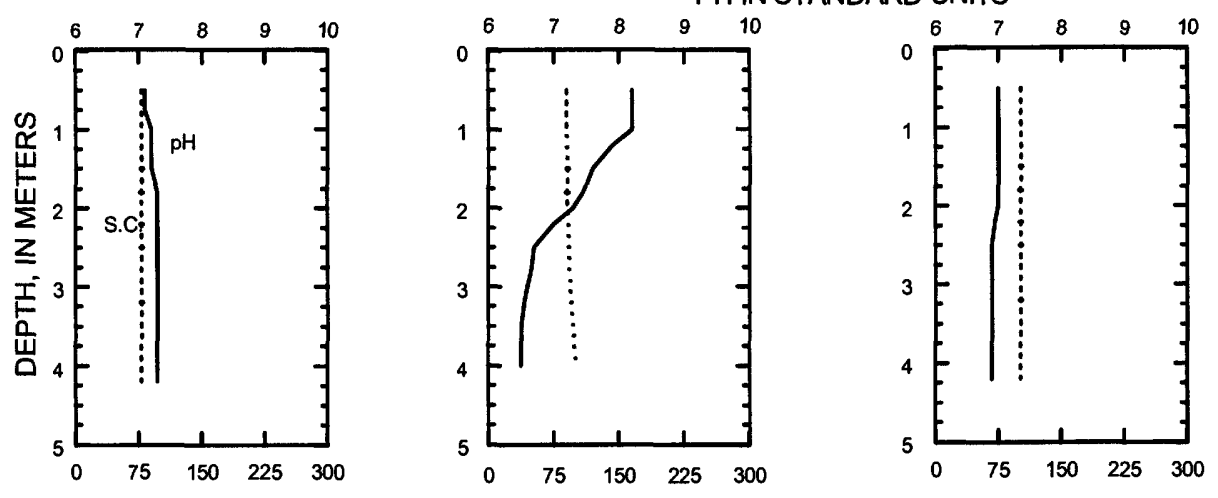

\begin{tabular}{ccccc}
\multicolumn{2}{c}{} & \multicolumn{1}{c}{ Auq-10 } & & \multicolumn{3}{c}{ Sep-21 } \\
& 5.03 & & \multicolumn{3}{c}{5.16} \\
& 1.2 & & 0.5 & \\
0.5 & & 4.5 & 1.5 & 4.2 \\
20.4 & 20.4 & 18.5 & 18.1 \\
102 & 102 & 101 & 101 \\
7.0 & 6.9 & 7.0 & 7.4 \\
6.1 & 6.0 & 7.9 & 7.6
\end{tabular}

8-10-04

9-21-04

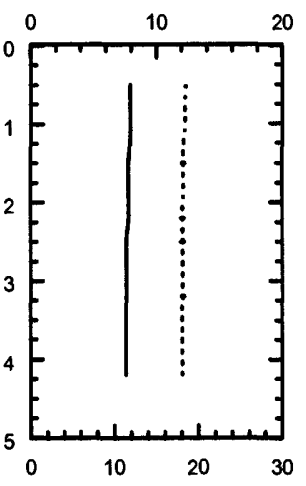

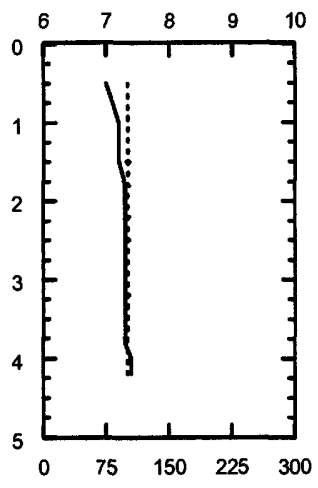

SPECIFIC CONDUCTANCE (S.C.) IN MICROSIEMENS PER CENTIMETER AT 25 DEGREES CELSIUS 

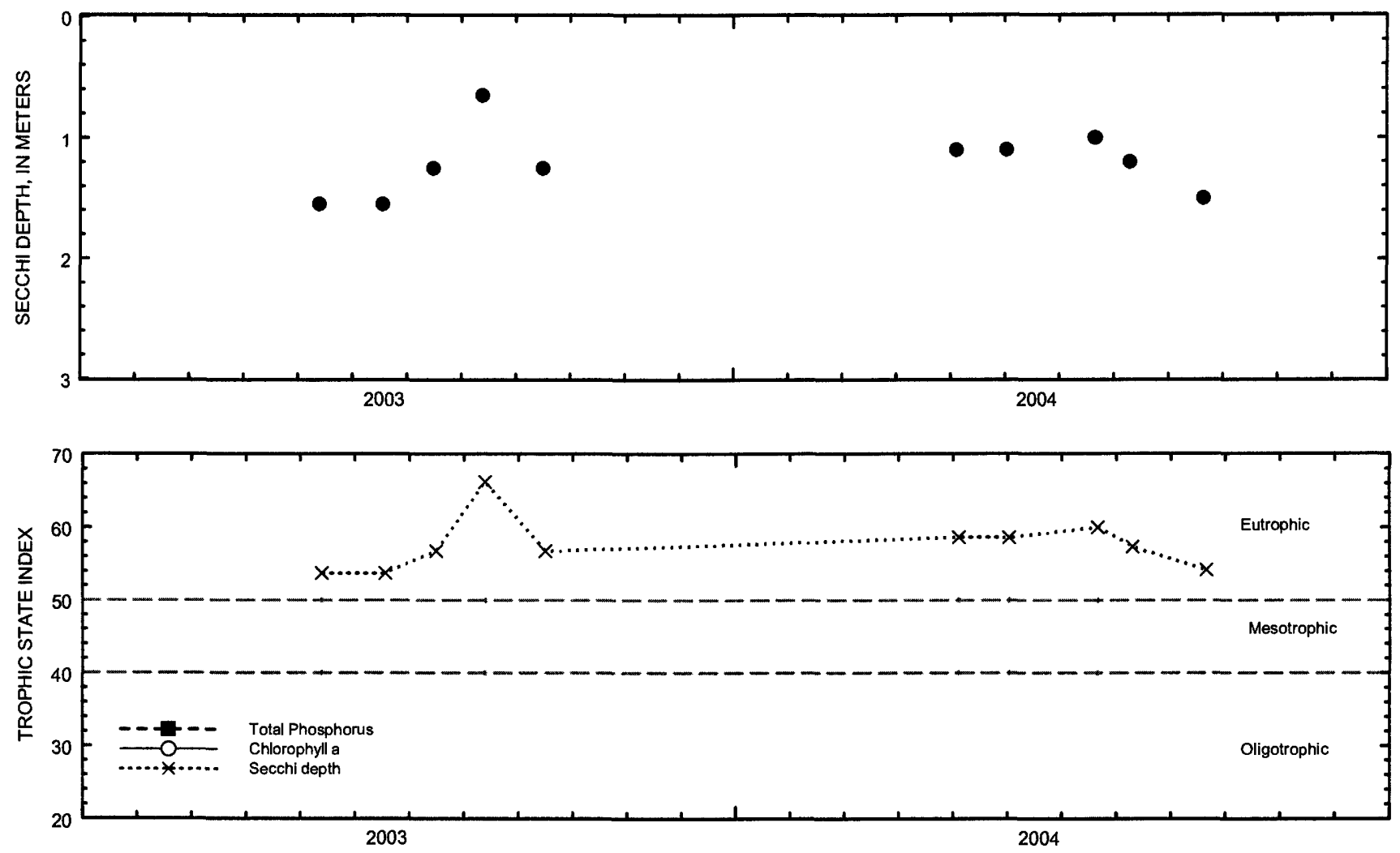

Secchi depths, and TSI data for Butternut Lake, Far South Site, near Park Falls, Wisconsin. 


\section{DELAVAN LAKE NEAR DELAVAN, WI}

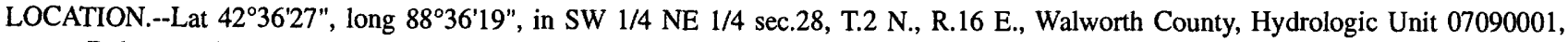
at Delavan Lake Sanitary District Lift Station No. 2 at Delavan Lake Yacht Club, 1.0 mi southeast of outlet, and 2.7 mi southeast of Delavan.

DRAINAGE AREA.-- $41.4 \mathrm{mi}^{2}$, of which $2.3 \mathrm{mi}^{2}$ is non-contributing. Area of Delavan Lake, 2,072 acres.

PERIOD OF RECORD.--October 1983 to current year. October 1983 to September 1985 data published in Water Resources Investigation series report "Water Quality and Hydrology of Delavan Lake in Southeastern Wisconsin" by S. J. Field and M. D. Duerk (1988).

GAGE.--Water-stage recorder. Datum of gage is $922.92 \mathrm{ft}$ above sea level. Prior to Sept. 5, 1989, staff gage at bridge on North Shore Drive at same datum.

REMARKS.--Lake was ice covered from Jan. 9 to Mar. 24. Lake levels controlled by Delavan Lake Sanitary District. Gage-height telemeter at station.

EXTREMES FOR PERIOD OF RECORD.--Maximum gage height observed, $6.19 \mathrm{ft}$, Feb. 21, 1994; minimum daily, $-4.44 \mathrm{ft}$, Nov. 6,1989 (lake drawn down for lake rehabilitation program).

EXTREMES FOR CURRENT YEAR.--Maximum gage height, $5.69 \mathrm{ft}$, May 23; minimum, $4.15 \mathrm{ft}$, Oct. 24.

GAGE HEIGHT, FEET

WATER YEAR OCTOBER 2003 TO SEPTEMBER 2004 DAILY MEAN VALUES

\begin{tabular}{|c|c|c|c|c|c|c|c|c|c|c|c|c|}
\hline DAY & OCT & NOV & DEC & JAN & FEB & MAR & APR & MAY & JUN & JUL & $A \cup G$ & SEP \\
\hline $\begin{array}{l}1 \\
2 \\
3 \\
4 \\
5\end{array}$ & $\begin{array}{l}4.26 \\
4.24 \\
4.23 \\
4.23 \\
4.22\end{array}$ & $\begin{array}{l}4.17 \\
4.28 \\
4.42 \\
4.57 \\
4.66\end{array}$ & $\begin{array}{l}4.78 \\
4.77 \\
4.77 \\
4.77 \\
4.79\end{array}$ & $\begin{array}{l}5.00 \\
5.00 \\
5.00 \\
5.00 \\
4.99\end{array}$ & $\begin{array}{l}4.96 \\
4.96 \\
4.98 \\
4.98 \\
4.98\end{array}$ & $\begin{array}{l}4.93 \\
4.95 \\
4.95 \\
4.95 \\
5.07\end{array}$ & $\begin{array}{l}4.97 \\
4.96 \\
4.96 \\
4.96 \\
4.95\end{array}$ & $\begin{array}{l}4.98 \\
4.98 \\
4.98 \\
4.97 \\
4.97\end{array}$ & $\begin{array}{l}5.11 \\
5.04 \\
5.00 \\
5.01 \\
5.03\end{array}$ & $\begin{array}{l}4.99 \\
4.99 \\
4.99 \\
5.05 \\
5.03\end{array}$ & $\begin{array}{l}4.85 \\
4.85 \\
4.86 \\
4.93 \\
4.91\end{array}$ & $\begin{array}{l}4.82 \\
4.80 \\
4.79 \\
4.79 \\
4.78\end{array}$ \\
\hline $\begin{array}{r}6 \\
7 \\
8 \\
9 \\
10\end{array}$ & $\begin{array}{l}4.21 \\
4.20 \\
4.20 \\
4.19 \\
4.19\end{array}$ & $\begin{array}{l}4.68 \\
4.67 \\
4.67 \\
4.66 \\
4.66\end{array}$ & $\begin{array}{l}4.78 \\
4.79 \\
4.79 \\
4.81 \\
5.00\end{array}$ & $\begin{array}{l}4.98 \\
4.97 \\
4.95 \\
4.95 \\
4.94\end{array}$ & $\begin{array}{l}5.00 \\
5.00 \\
5.00 \\
4.99 \\
4.99\end{array}$ & $\begin{array}{l}5.03 \\
4.96 \\
4.95 \\
4.95 \\
4.94\end{array}$ & $\begin{array}{l}4.94 \\
4.95 \\
4.96 \\
4.96 \\
4.96\end{array}$ & $\begin{array}{l}4.98 \\
4.97 \\
4.99 \\
5.03 \\
5.05\end{array}$ & $\begin{array}{l}5.05 \\
5.06 \\
5.06 \\
5.06 \\
5.08\end{array}$ & $\begin{array}{l}5.00 \\
5.01 \\
5.00 \\
4.99 \\
4.98\end{array}$ & $\begin{array}{l}4.89 \\
4.88 \\
4.88 \\
4.87 \\
4.85\end{array}$ & $\begin{array}{l}4.78 \\
4.77 \\
4.76 \\
4.75 \\
4.75\end{array}$ \\
\hline $\begin{array}{l}11 \\
12 \\
13 \\
14 \\
15\end{array}$ & $\begin{array}{l}4.19 \\
4.21 \\
4.20 \\
4.23 \\
4.23\end{array}$ & $\begin{array}{l}4.66 \\
4.66 \\
4.65 \\
4.65 \\
4.64\end{array}$ & $\begin{array}{l}5.09 \\
5.11 \\
5.11 \\
5.12 \\
5.12\end{array}$ & $\begin{array}{l}4.94 \\
4.93 \\
4.93 \\
4.94 \\
4.93\end{array}$ & $\begin{array}{l}4.99 \\
4.99 \\
4.99 \\
4.98 \\
4.98\end{array}$ & $\begin{array}{l}4.94 \\
4.94 \\
4.93 \\
4.94 \\
4.94\end{array}$ & $\begin{array}{l}4.96 \\
4.96 \\
4.95 \\
4.95 \\
4.95\end{array}$ & $\begin{array}{l}5.05 \\
5.04 \\
5.11 \\
5.23 \\
5.29\end{array}$ & $\begin{array}{l}5.11 \\
5.09 \\
5.02 \\
4.97 \\
5.01\end{array}$ & $\begin{array}{l}4.96 \\
4.97 \\
4.97 \\
4.97 \\
4.97\end{array}$ & $\begin{array}{l}4.84 \\
4.82 \\
4.81 \\
4.80 \\
4.79\end{array}$ & $\begin{array}{l}4.74 \\
4.73 \\
4.73 \\
4.72 \\
4.72\end{array}$ \\
\hline $\begin{array}{l}16 \\
17 \\
18 \\
19 \\
20\end{array}$ & $\begin{array}{l}4.21 \\
4.20 \\
4.19 \\
4.18 \\
4.18\end{array}$ & $\begin{array}{l}4.64 \\
4.65 \\
4.72 \\
4.76 \\
4.76\end{array}$ & $\begin{array}{r}5.11 \\
5.11 \\
e 5.10 \\
5.09 \\
5.08\end{array}$ & $\begin{array}{l}4.93 \\
4.96 \\
4.97 \\
4.96 \\
4.96\end{array}$ & $\begin{array}{l}4.98 \\
4.97 \\
4.97 \\
4.97 \\
4.98\end{array}$ & $\begin{array}{l}4.93 \\
4.93 \\
4.94 \\
4.94 \\
4.94\end{array}$ & $\begin{array}{l}4.95 \\
4.98 \\
4.98 \\
4.98 \\
5.00\end{array}$ & $\begin{array}{l}5.24 \\
5.18 \\
5.16 \\
5.13 \\
5.15\end{array}$ & $\begin{array}{l}5.03 \\
5.12 \\
5.17 \\
5.09 \\
5.00\end{array}$ & $\begin{array}{l}4.98 \\
4.96 \\
4.95 \\
4.94 \\
4.93\end{array}$ & $\begin{array}{l}4.78 \\
4.78 \\
4.78 \\
4.77 \\
4.76\end{array}$ & $\begin{array}{l}4.72 \\
4.71 \\
4.70 \\
4.69 \\
4.68\end{array}$ \\
\hline $\begin{array}{l}21 \\
22 \\
23 \\
24 \\
25\end{array}$ & $\begin{array}{l}4.18 \\
4.17 \\
4.16 \\
4.17 \\
4.20\end{array}$ & $\begin{array}{l}4.77 \\
4.77 \\
4.79 \\
4.79 \\
4.79\end{array}$ & $\begin{array}{l}5.06 \\
5.05 \\
5.04 \\
5.03 \\
5.02\end{array}$ & $\begin{array}{l}4.96 \\
4.96 \\
4.96 \\
4.96 \\
4.96\end{array}$ & $\begin{array}{l}5.00 \\
5.01 \\
5.03 \\
5.04 \\
5.03\end{array}$ & $\begin{array}{l}4.94 \\
4.93 \\
4.93 \\
4.97 \\
5.00\end{array}$ & $\begin{array}{l}5.07 \\
5.04 \\
5.00 \\
4.98 \\
4.98\end{array}$ & $\begin{array}{l}5.18 \\
5.46 \\
5.66 \\
5.65 \\
5.54\end{array}$ & $\begin{array}{l}4.98 \\
5.03 \\
5.00 \\
5.00 \\
5.01\end{array}$ & $\begin{array}{l}4.95 \\
4.98 \\
4.98 \\
4.96 \\
4.94\end{array}$ & $\begin{array}{l}4.75 \\
4.73 \\
4.73 \\
4.74 \\
4.76\end{array}$ & $\begin{array}{l}4.66 \\
4.65 \\
4.64 \\
4.63 \\
4.62\end{array}$ \\
\hline $\begin{array}{l}26 \\
27 \\
28 \\
29 \\
30 \\
31\end{array}$ & $\begin{array}{l}4.19 \\
4.18 \\
4.18 \\
4.17 \\
4.17 \\
4.18\end{array}$ & $\begin{array}{r}4.78 \\
4.79 \\
4.79 \\
4.79 \\
4.78 \\
-.\end{array}$ & $\begin{array}{l}5.01 \\
5.00 \\
5.02 \\
5.03 \\
5.02 \\
5.01\end{array}$ & $\begin{array}{l}4.96 \\
4.97 \\
4.97 \\
4.97 \\
4.97 \\
4.97\end{array}$ & $\begin{array}{r}5.01 \\
4.97 \\
4.94 \\
4.92 \\
\ldots- \\
\ldots .\end{array}$ & $\begin{array}{l}5.10 \\
5.12 \\
5.11 \\
5.14 \\
5.09 \\
5.03\end{array}$ & $\begin{array}{r}4.98 \\
4.97 \\
4.94 \\
4.94 \\
4.96 \\
\ldots\end{array}$ & $\begin{array}{l}5.39 \\
5.27 \\
5.14 \\
5.05 \\
5.02 \\
5.12\end{array}$ & $\begin{array}{r}5.02 \\
5.03 \\
5.03 \\
5.02 \\
5.01 \\
\ldots\end{array}$ & $\begin{array}{l}4.92 \\
4.91 \\
4.90 \\
4.89 \\
4.88 \\
4.87\end{array}$ & $\begin{array}{l}4.76 \\
4.78 \\
4.82 \\
4.83 \\
4.83 \\
4.83\end{array}$ & $\begin{array}{r}4.61 \\
4.61 \\
4.59 \\
4.58 \\
4.58 \\
--.\end{array}$ \\
\hline $\begin{array}{r}\text { MEAN } \\
\text { MAX } \\
M I N\end{array}$ & $\begin{array}{l}4.20 \\
4.26 \\
4.16\end{array}$ & $\begin{array}{l}4.67 \\
4.79 \\
4.17\end{array}$ & $\begin{array}{l}4.98 \\
5.12 \\
4.77\end{array}$ & $\begin{array}{l}4.96 \\
5.00 \\
4.93\end{array}$ & $\begin{array}{l}4.99 \\
5.04 \\
4.92\end{array}$ & $\begin{array}{l}4.98 \\
5.14 \\
4.93\end{array}$ & $\begin{array}{l}4.97 \\
5.07 \\
4.94\end{array}$ & $\begin{array}{l}5.16 \\
5.66 \\
4.97\end{array}$ & $\begin{array}{l}5.04 \\
5.17 \\
4.97\end{array}$ & $\begin{array}{l}4.96 \\
5.05 \\
4.87\end{array}$ & $\begin{array}{l}4.81 \\
4.93 \\
4.73\end{array}$ & $\begin{array}{l}4.70 \\
4.82 \\
4.58\end{array}$ \\
\hline
\end{tabular}

e Estim ated

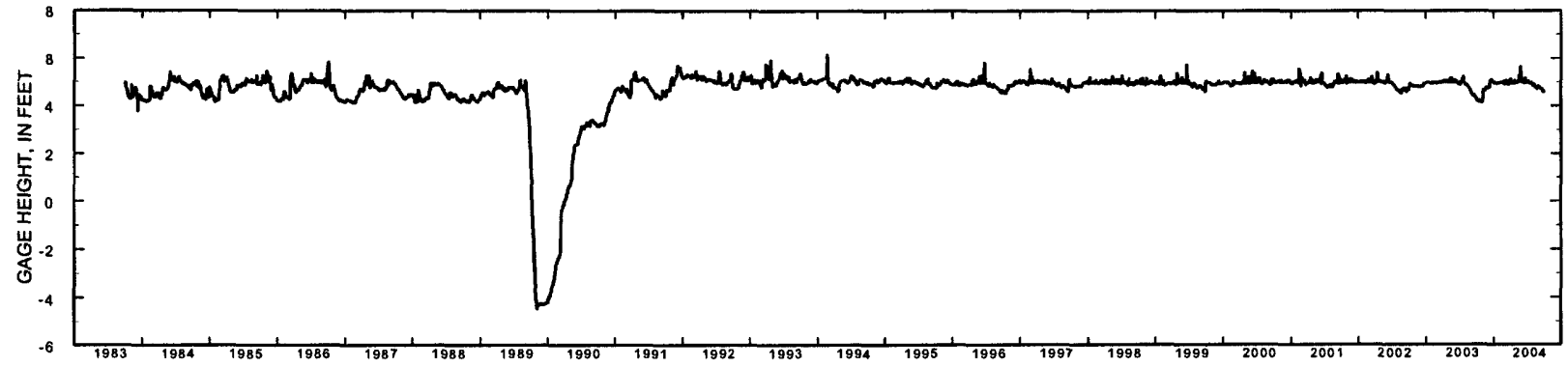




\section{DELAVAN LAKE AT CENTER NEAR DELAVAN LAKE, WI}

LOCATION.--Lat $42^{\circ} 35^{\prime} 56^{\prime \prime}$, long $88^{\circ} 36^{\prime} 50^{\prime \prime}$, in SE 1/4 SW 1/4 sec.28, T.2 N., R.16 E., Walworth County, Hydrologic Unit 07090001, 2.6 mi southeast of Delavan.

DRAINAGE AREA.--41.4 mi ${ }^{2}$, of which $2.3 \mathrm{mi}^{2}$ is non-contributing. Area of Delavan Lake, 2,072 acres.

PERIOD OF RECORD.--October 1983 to current year.

REMARKS.--Lake ice-covered during February measurements. Water-quality analyses done by the U.S. Geological Survey National Water Quality Laboratory. Samples for determination of chlorophyll $a$ concentration are collected from the top $0.5 \mathrm{~m}$ of the lake.

WATER-QUALITY DATA, NOVEMBER 17, 2003 TO MAY 19, 2004

(Milligrams per liter unless otherwise indicated)

Date

Secchi depth $(\mathrm{m})$

Depth of sample (m)

Chlorophyll a, phytoplankton ( $\mu \mathrm{g} / \mathrm{L}$ )

Water temperature $\left({ }^{\circ} \mathrm{C}\right)$

Specific conductance $(\mu \mathrm{S} / \mathrm{cm})$

$\mathrm{pH}$

Phosphorus, total (as P)

Phosphorus, ortho, dissolved (as P)

Nitrogen, $\mathrm{NO}_{2}+\mathrm{NO}_{3}$, diss. (as $\mathrm{N}$ )

Nitrogen, ammonia, dissolved (as $\mathrm{N}$ )

Nitrogen, amm. + org., total (as N)

Nitrogen, total (as $\mathrm{N}$ )

Color (Pt-Co. scale)

Turbitdity (NTU)

Hardness, as $\mathrm{CaCO}_{3}$

Magnesium, dissolved (Mg)

Sodium, dissolved $(\mathrm{Na})$

Potassium, dissolved (K)

Alkalinity, as $\mathrm{CaCO}_{3}$

Sulfate, dissolved $\left(\mathrm{SO}_{4}\right)$

Chloride, dissolved $(\mathrm{Cl})$

Silica, dissolved $\left(\mathrm{SiO}_{2}\right)$

Solids, dissolved, at $180^{\circ} \mathrm{C}$

Iron, dissolved $(\mathrm{Fe})(\mu \mathrm{g} / \mathrm{L})$

Manganese, dissolved, $(\mathrm{Mn})(\mu \mathrm{g} / \mathrm{L})$
Lake stage ( $\mathrm{ft}$ )

Dissolved oxygen (mg/L)

Calcium, dissolved (Ca)

\begin{tabular}{|c|c|c|}
\hline & & \\
\hline 0.5 & 16 & 0.5 \\
\hline 3.4 & -- & 6.2 \\
\hline 7.7 & 7.4 & 0.7 \\
\hline 562 & 564 & 571 \\
\hline 8.1 & 8.1 & 8.8 \\
\hline 10.4 & 9.3 & 15.1 \\
\hline 0.102 & 0.116 & 0.086 \\
\hline 0.074 & 0.074 & 0.052 \\
\hline 0.072 & -- & 0.206 \\
\hline 0.133 & -- & 0.051 \\
\hline 0.78 & - & 0.66 \\
\hline 0.85 & -- & 0.87 \\
\hline-- & - & -. \\
\hline-- & -- & - \\
\hline-- & -- & - \\
\hline-- & - & -- \\
\hline- & - & - \\
\hline- & -- & -- \\
\hline -- & -- & - \\
\hline -- & - & - \\
\hline-- & -- & - \\
\hline- & - & - \\
\hline -- & -- & -- \\
\hline- & -- & -- \\
\hline$\cdots$ & - & -- \\
\hline
\end{tabular}

2-25-04

\begin{tabular}{|c|c|c|}
\hline & & \\
\hline 5 & 10 & 16 \\
\hline- & -- & -- \\
\hline 1.4 & 1.7 & 2.9 \\
\hline 616 & 622 & 746 \\
\hline 8.8 & 8.4 & 7.7 \\
\hline 14.5 & 11.8 & 5.1 \\
\hline 0.087 & 0.088 & 0.175 \\
\hline- & - & 0.143 \\
\hline -- & - & - \\
\hline -- & - & - \\
\hline -- & -- & - \\
\hline -- & - & - \\
\hline -- & -- & -- \\
\hline -- & - & - \\
\hline - & -- & - \\
\hline- & -- & -- \\
\hline-- & -- & - \\
\hline -- & - & - \\
\hline-- & - & -- \\
\hline -- & -- & - \\
\hline- & - & -- \\
\hline- & -- & - \\
\hline- & -- & - \\
\hline- & - & -- \\
\hline-- & - & - \\
\hline - & -- & -- \\
\hline
\end{tabular}

\begin{tabular}{cccc}
\multicolumn{2}{c}{ Apr-13 } & \multicolumn{2}{c}{ May-19 } \\
\multicolumn{2}{c}{3.93 .4} & \multicolumn{2}{c}{5.0} \\
0.5 & 16 & 0.5 & 16 \\
8.2 & - & 8.8 & - \\
7.8 & 7.4 & 16.5 & 12.5 \\
566 & 567 & 561 & 577 \\
8.4 & 8.5 & 8.5 & 8.4 \\
13.3 & 12.8 & 9.9 & 5.8 \\
0.046 & 0.051 & 0.059 & 0.086 \\
0.009 & $<0.006$ & 0.020 & 0.050 \\
0.059 & 0.059 & 0.063 & - \\
$<0.010$ & $<0.010$ & 0.024 & -- \\
0.65 & 0.68 & 0.69 & - \\
0.71 & 0.74 & 0.75 & - \\
10 & 10 & -- & - \\
$<2.0$ & $<2.0$ & - & - \\
230 & 230 & -- & - \\
34.9 & 34.9 & -- & - \\
33.6 & 33.6 & -- & - \\
27 & 27 & -- & - \\
2.73 & 2.76 & - & - \\
182 & 182 & -- & - \\
23.2 & 23.5 & -- & - \\
58.7 & 60.1 & - & - \\
0.1 & 0.2 & - & - \\
335 & 333 & - & - \\
$<6$ & $<6$ & -- & - \\
1 & 0.9 &.- & -
\end{tabular}

DISSOLVED OXYGEN (D.O.) IN MILLIGRAMS PER LITER
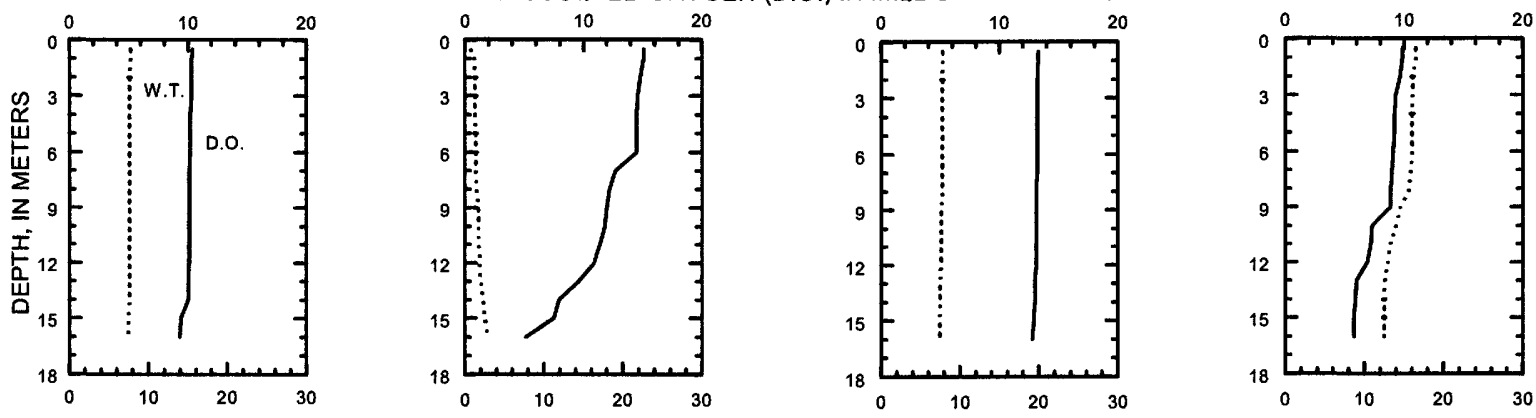

WATER TEMPERATURE (W.T.) IN DEGREES CELSIUS
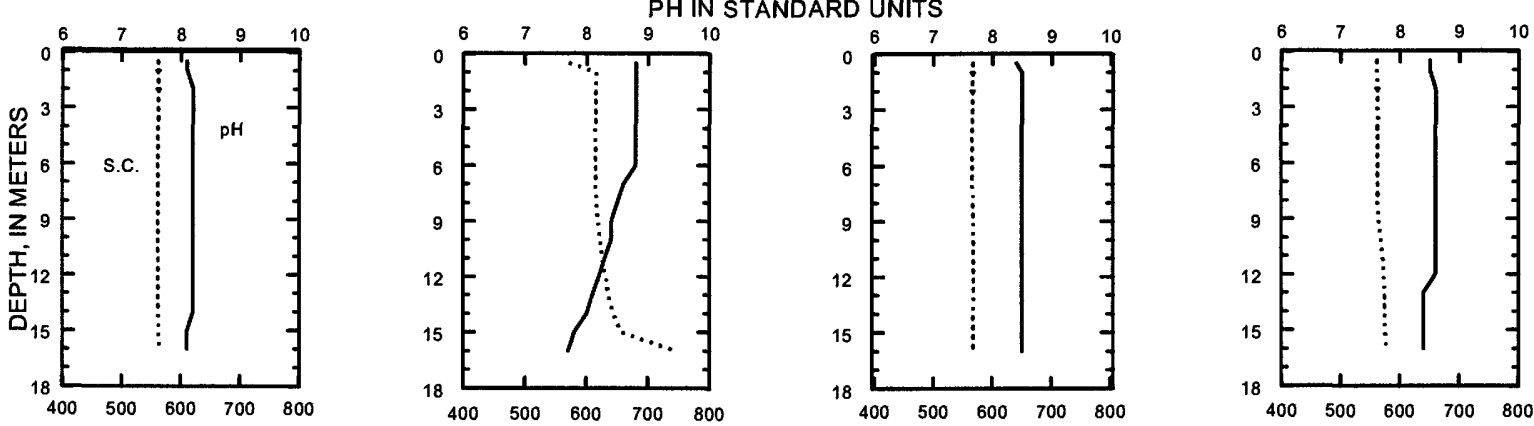

SPECIFIC CONDUCTANCE (S.C.) IN MICROSIEMENS PER CENTIMETER AT 25 DEGREES CELSIUS 
WATER-QUALITY DATA, JUNE 22 TO JULY 20, 2004

(Milligrams per liter unless otherwise indicated)

Date

Lake stage $(\mathrm{ft})$

Secchi depth $(\mathrm{m})$

Depth of sample $(\mathrm{m})$

Chlorophyll a, phytoplankton ( $\mu \mathrm{g} / \mathrm{L})$

Water temperature $\left({ }^{\circ} \mathrm{C}\right)$

Specific conductance $(\mu \mathrm{S} / \mathrm{cm})$

$\mathrm{pH}$

Dissolved oxygen (mg/L)

Phosphorus, total (as P)

Phosphorus, ortho, dissolved (as $\mathrm{P}$ )

Nitrogen, $\mathrm{NO}_{2}+\mathrm{NO}_{3}$, diss. (as $\mathrm{N}$ )

Nitrogen, ammonia, dissolved (as $\mathrm{N}$ )

Nitrogen, amm. + org., total (as N)

Nitrogen, total (as N)

\begin{tabular}{|c|c|c|c|c|c|c|c|}
\hline & & & & & & & \\
\hline 0.5 & 8 & 12 & 16 & 0.5 & 6 & 13 & 16 \\
\hline 30.3 & - & - & - & 10.4 & - & - & - \\
\hline 21.5 & 20.3 & 15.9 & 14.3 & 24.7 & 23.2 & 15 & 14.3 \\
\hline 543 & 552 & 573 & 591 & 513 & 526 & 591 & 608 \\
\hline 8.5 & 8.3 & 8.1 & 8.0 & 9.0 & 8.9 & 9.0 & 8.9 \\
\hline 10.1 & 5.8 & 1.9 & 0.2 & 11.2 & 7.6 & 0.2 & 0.2 \\
\hline 0.063 & 0.068 & 0.132 & 0.260 & 0.031 & 0.026 & 0.360 & 0.470 \\
\hline 0.005 & 0.034 & 0.099 & 0.213 & $<0.006$ & 0.003 & 0.305 & 0.387 \\
\hline 0.097 & - & - & - & $<0.016$ & - & - & - \\
\hline 0.042 & - & - & - & 0.017 & - & - & - \\
\hline 0.96 & - & -. & - & 0.82 & - & - & - \\
\hline 1.1 & - & -- & - & -- & - & - & - \\
\hline
\end{tabular}

6-22-04

7-20-04

DISSOLVED OXYGEN (D.O.) IN MILLIGRAMS PER LITER
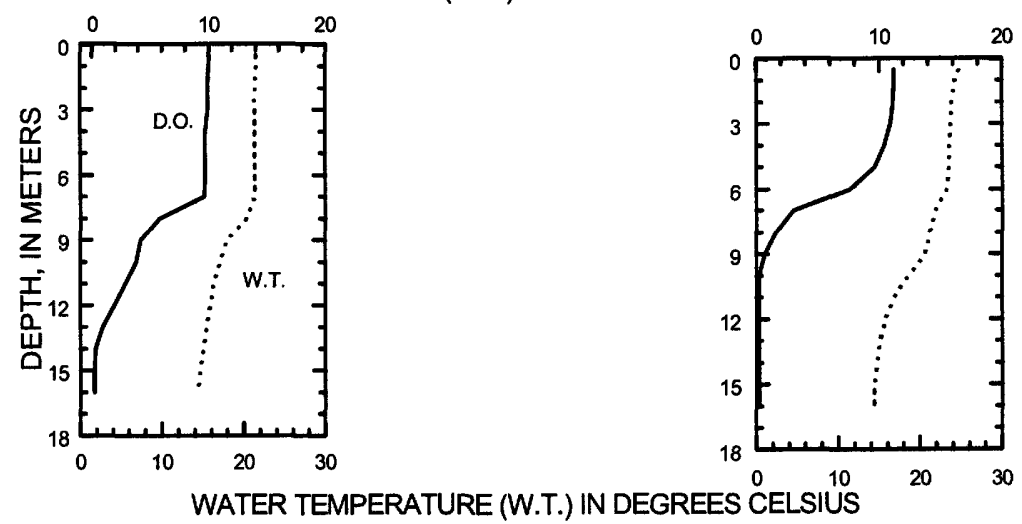

PH IN STANDARD UNITS
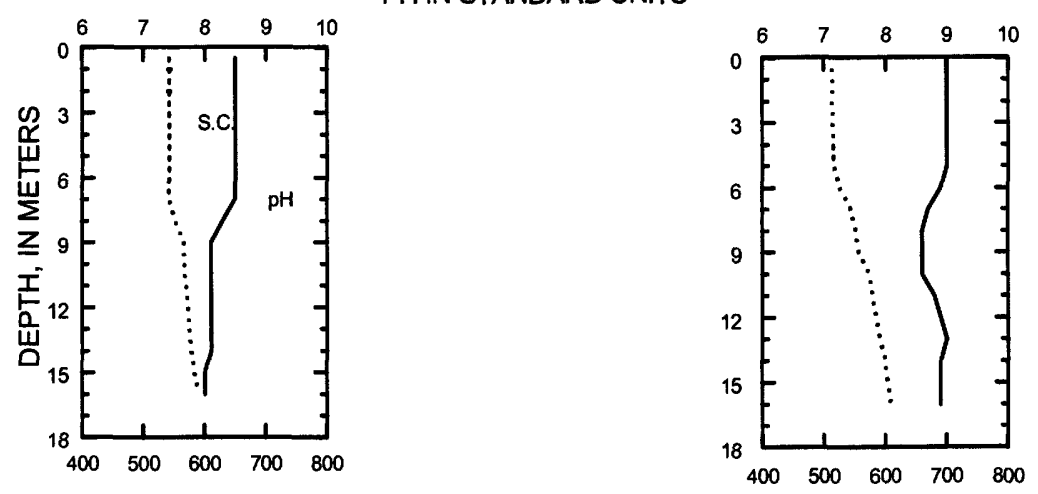

SPECIFIC CONDUCTANCE (S.C.) IN MICROSIEMENS PER CENTIMETER AT 25 DEGREES CELSIUS 
WATER-QUALITY DATA, AUGUST 19, 2004

(Milligrams per liter unless otherwise indicated)
Date

Lake stage (ft)

Secchi depth $(m)$

Depth of sample $(m)$

Chlorophyll a, phytoplankton ( $\mu \mathrm{g} / \mathrm{L}$ )

Water temperature $\left({ }^{\circ} \mathrm{C}\right)$

Specific conductance $(\mu \mathrm{S} / \mathrm{cm})$

Dissolved oxygen (mg/L)

Phosphorus, total (as P)

Phosphorus, ortho, dissolved (as $\mathbf{P}$ )

Nitrogen, $\mathrm{NO}_{2}+\mathrm{NO}_{3}$, diss. (as $\mathrm{N}$ )

Nitrogen, ammonia, dissolved (as $\mathrm{N}$ )

Nitrogen, amm. + org., total (as N)

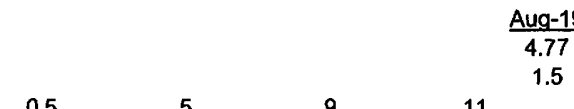

$\begin{array}{ccc}0.5 & 5 & 9 \\ 20.6 & -- & - \\ 21.8 & 20.7 & 20.6 \\ 526 & 529 & 533 \\ 8.6 & 8.4 & 8.4 \\ 9.6 & 7.5 & 6.5 \\ 0.088 & 0.072 & 0.069 \\ 0.012 & - & 0.028 \\ 0.008 & - & - \\ 0.042 & -- & - \\ 0.82 & - & -\end{array}$

8-19-04

DISSOLVED OXYGEN (D.O.) IN MILLIGRAMS PER LITER

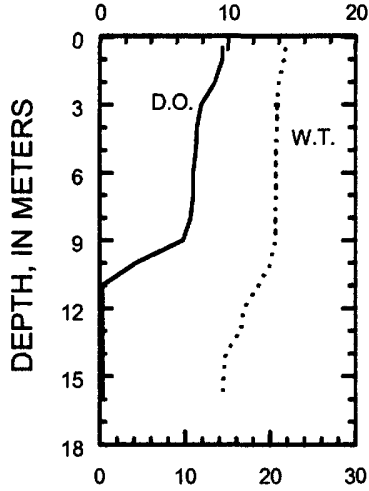

WATER TEMPERATURE (W.T.) IN DEGREES CELSIUS

PHIN STANDARD UNITS

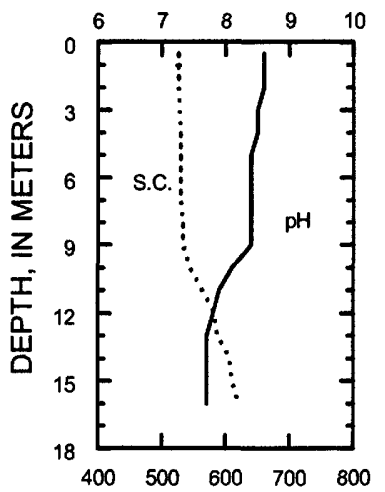

SPECIFIC CONDUCTANCE (S.C.) IN MICROSIEMENS PER CENTIMETER AT 25 DEGREES CELSIUS 


\section{DELAVAN LAKE AT CENTER NEAR DELAVAN LAKE, WI--CONTINUED}

WATER-QUALITY DATA, SEPTEMBER 22, 2004

(Milligrams per liter unless otherwise indicated)

Date

Lake stage (ft)

Secchi depth $(m)$

Depth of sample (m)

Chlorophyll a, phytoplankton ( $\mu \mathrm{g} / \mathrm{L})$

Water temperature $\left({ }^{\circ} \mathrm{C}\right)$

Speciflc conductance $(\mu \mathrm{S} / \mathrm{cm})$

$\mathrm{pH}$

Dissolved oxygen (mg/L)

Phosphorus, total (as P)

Phosphorus, ortho, dissolved (as $P$ )

Nitrogen, $\mathrm{NO}_{2}+\mathrm{NO}_{3}$, diss. (as $\mathrm{N}$ )

Nitrogen, ammonia, dissolved (as $\mathrm{N}$ )

Nitrogen, amm. + org., total (as N)

\begin{tabular}{|c|c|c|c|}
\hline \multicolumn{4}{|c|}{$\begin{array}{c}\text { Sep-22 } \\
4.68 \\
1.7\end{array}$} \\
\hline 0.5 & 12 & 14 & 16 \\
\hline 25.0 & - & - & - \\
\hline 21.2 & 20.0 & 16.4 & 14.9 \\
\hline 525 & 545 & 600 & 636 \\
\hline 8.8 & 8.2 & 7.9 & 7.8 \\
\hline 8.6 & 0.7 & 0.2 & 0.2 \\
\hline 0.107 & 0.156 & 0.380 & 0.710 \\
\hline 0.044 & 0.097 & 0.355 & 0.683 \\
\hline 0.011 & - & - & - \\
\hline 0.041 & - & - & - \\
\hline 0.94 & - & -- & - \\
\hline
\end{tabular}

9-22-04

DISSOLVED OXYGEN (D.O.) IN MILIGRAMS PER LITER

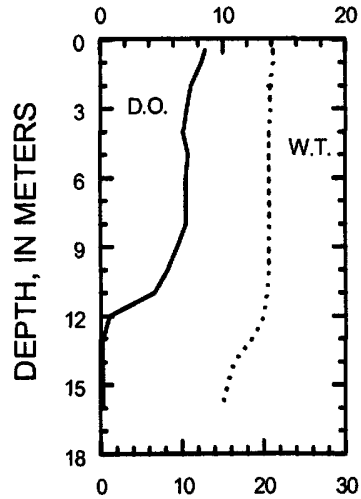

WATER TEMPERATURE (W.T.) IN DEGREES CELSIUS

PH IN STANDARD UNITS

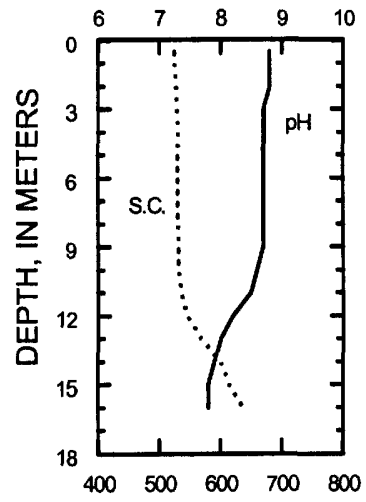

SPECIFIC CONDUCTANCE (S.C.) IN MICROSIEMENS PER CENTIMETER AT 25 DEGREES CELSIUS 

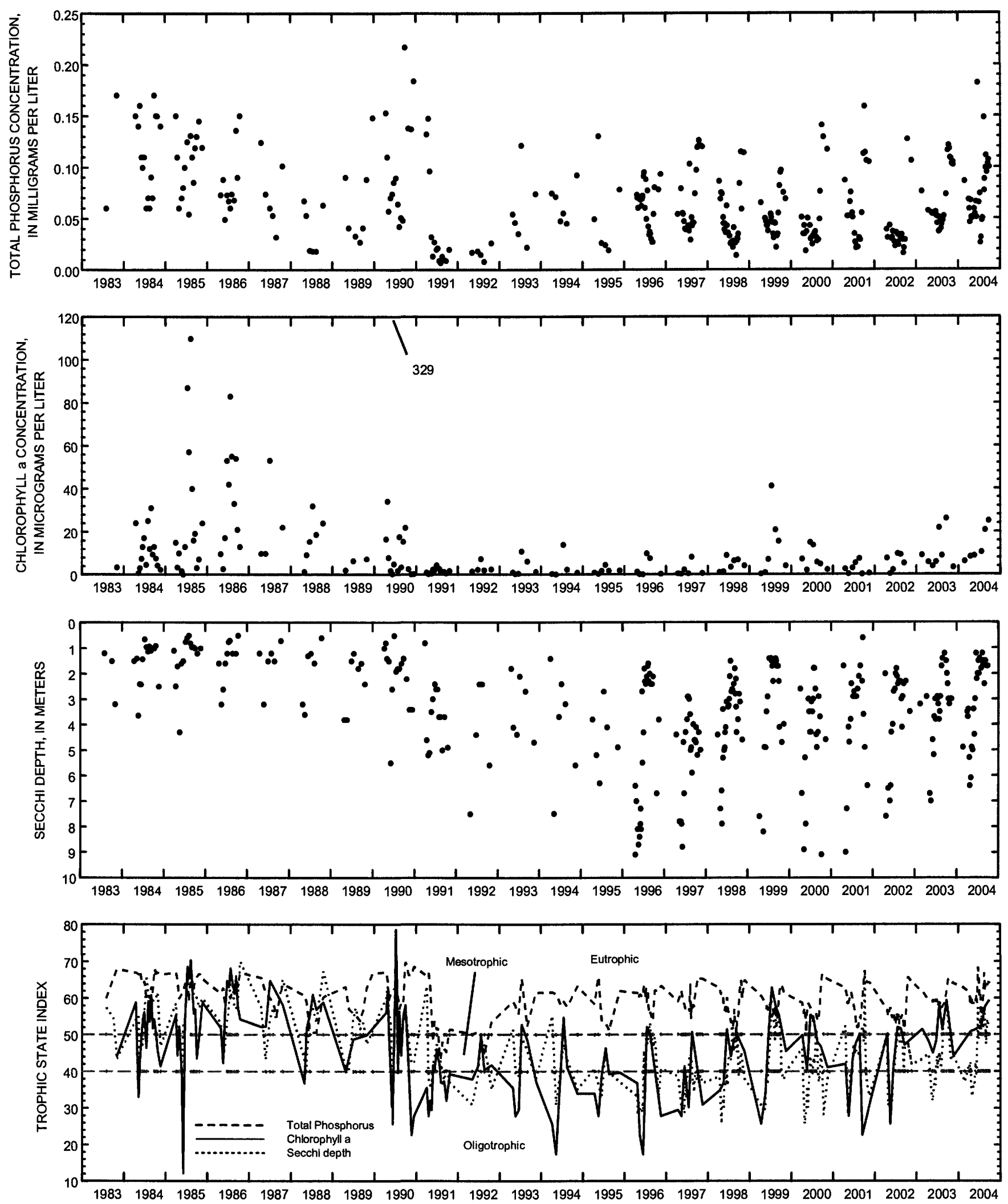

Surface total phosphorus, chlorophyll a concentrations, Secchi depths, and TSI data for Delavan Lake, at Center, near Delavan, Wisconsin. 
ADDITIONAL WATER-QUALITY DATA, OCTOBER 1, 2003 TO SEPTEMBER 30, 2004*

(Milligrams per liter unless otherwise indicated)

Lake stage ( $\mathrm{ft}$ )

Secchi-depth (meters)

Depth of sample (meters)

Water temperature $\left({ }^{\circ} \mathrm{C}\right)$

Phosphorus, total (as P)

Lake stage ( $\mathrm{ft}$ )

Secchi-depth (meters)

Depth of sample (meters)

Water temperature $\left({ }^{\circ} \mathrm{C}\right)$

Phosphorus, total (as P)

Lake stage ( $\mathrm{ft}$ )

Secchi-depth (meters)

Depth of sample (meters)

Water temperature $\left({ }^{\circ} \mathrm{C}\right)$

Phosphorus, total (as P)

Lake stage ( $\mathrm{ft}$ )

Secchi-depth (meters)

Depth of sample (meters)

Water temperature $\left({ }^{\circ} \mathrm{C}\right)$

Phosphorus, total (as P)

Lake stage (ft)

Secchi-depth (meters)

Depth of sample (meters)

Water temperature $\left({ }^{\circ} \mathrm{C}\right)$

Phosphorus, total (as P)

Lake stage ( $\mathrm{ft}$ )

Secchi-depth (meters)

Depth of sample (meters)

Water temperature $\left({ }^{\circ} \mathrm{C}\right)$

Phosphorus, total (as P)

$\begin{array}{ccccc}\text { Oct. } 1 & \text { Oct. } 8 & \text { Oct. } 17 & \text { Oct. } 22 & \text { Oct. } 30 \\ 4.26 & 4.20 & 4.20 & 4.17 & 4.17 \\ 1.5 & 2.0 & 2.4 & 3.0 & 3.2 \\ 0.5 & 0.5 & 0.5 & 0.5 & 0.5 \\ 15.0 & 14.0 & 14.0 & 13.5 & 12.0 \\ 0.116 & 0.121 & 0.118 & 0.109 & 0.108\end{array}$

$\begin{array}{ccccc}\text { Nov.6 } & \text { Nov. 14 } & \text { Aprill } & \text { Aprill } & \text { April 22 } \\ 4.68 & 4.65 & 4.97 & 4.95 & 5.04 \\ 3.0 & 3.0 & 3.5 & 3.7 & 5.3 \\ 0.5 & 0.5 & 0.5 & 0.5 & 0.5 \\ 10.0 & 7.5 & 6.5 & 8.0 & 11.0 \\ 0.103 & 0.105 & 0.068 & 0.059 & 0.067\end{array}$

April 27

4.97

6.4

0.5

11.0

0.051

Lune 9

5.06

3.0

0.5

22.0

0.051

July 15
4.97
2.0
0.5
24.0
0.026

Aug 27

4.78

2.4

0.5

22.0

0.099

May 4
4.97
6.1
0.5
12.0
0.050

Lune 18

5.17

1.2

0.5

24.5

0.182

July 28
4.90
1.5
0.5
24.0
0.048

Aug 31

4.83

2.4

0.5

23.0

0.111
May 11

5.05

4.9

0.5

15.0

0.051

June 22

5.03

2.6

0.5

21.5

0.056

Aug. 2

4.85

1.8

0.5

24.0

0.050

$\frac{\text { Sept. }}{4.75}$
1.5
0.5
23.0
0.095

$\begin{array}{cc}\text { May 26 } & \text { June 4 } \\ 5.39 & 5.01 \\ 3.4 & 4.4 \\ 0.5 & 0.5 \\ 17.8 & 18.0 \\ 0.054 & 0.066\end{array}$

Luly 1

4.99

2.0

0.5

23.0

0.065

Aug 13

4.81

1.2

0.5

21.0

0.148

Sept. 17

4.71

1.7

0.5

21.0

0.103

July 9

0.074

* Measurements and samples collected by the Delavan Lake Sanitary District. 
LOCATION.--Lat $42^{\circ} 36^{\prime} 59^{\prime \prime}$, long $88^{\circ} 35^{\prime} 44^{\prime \prime}$, in NW 1/4 SW 1/4, sec.22, T.2 N., R.16 E., Walworth County, Hydrologic Unit 07090001, 2.6 mi southeast of Delavan.

DRAINAGE AREA.--41.4 $\mathrm{mi}^{2}$, of which $2.3 \mathrm{mi}^{2}$ is non-contributing.

PERIOD OF RECORD.--October 1983 to current year.

WATER-QUALITY DATA, APRIL 13 TO AUGUST 19, 2004

\begin{tabular}{lccccc} 
& April 13 & May 19 & June 22 & July 20 & Aug. 19 \\
\hline Secchi depth (meters) & 3.4 & 5.3 & -2.2 & 1.3 & 1.3
\end{tabular}

423526088380101 DELAVAN LAKE, AT SW END, NEAR DELAVAN LAKE, WI

LOCATION.--Lat $42^{\circ} 35^{\prime} 26^{\prime \prime}$, long $88^{\circ} 38^{\prime} 01^{\prime \prime}$, in SE $1 / 4$ NW 1/4, sec.32, T.2 N., R.16 E., Walworth County, Hydrologic Unit 07090001 , 2.6 mi southeast of Delavan.

DRAINAGE AREA.--41.4 $\mathrm{mi}^{2}$, of which $2.3 \mathrm{mi}^{2}$ is non-contributing.

PERIOD OF RECORD.--October 1983 to current year.

WATER-QUALITY DATA, APRIL 13 TO AUGUST 19, 2004

\begin{tabular}{lccccc} 
& April 13 & May 19 & June 22 & July 20 & Aug. 19 \\
\cline { 2 - 3 } & $-\cdots$ & -1.2 & 1.4 & 1.7
\end{tabular}




\section{LAKE DESAIR NEAR RICE LAKE, WI}

LOCATION.--Lat $45^{\circ} 34^{\prime} 46^{\prime \prime}$, long 91 $466^{\prime} 51^{\prime \prime}$, in SE 1/4 SW 1/4 NW 1/4 sec.6, T.35 N., R.11 W., Barron County, Hydrologic Unit 07050007, at public boat landing off 18 th Street about 3 miles northwest of the city of Rice Lake.

PERIOD OF RECORD.--August 2004 (discontinued). Data collected through Wis. Dept. of Natural Resources Self-Help Monitoring program since April 1993 are available.

REMARKS.--Lake sampled at deepest location. Water-quality analyses done by Wisconsin State Laboratory of Hygiene.

WATER-QUALITY DATA, AUGUST 17, 2004

(Milligrams per liter unless otherwise indicated)

Date

Secchi depth (m)

Depth of sample $(\mathrm{m})$

Chlorophyll a, phytoplankton $(\mu \mathrm{g} / \mathrm{L})$

Water temperature $\left({ }^{\circ} \mathrm{C}\right)$

Specific conductance $(\mu \mathrm{S} / \mathrm{cm})$

$\mathrm{pH}$

Dissolved oxygen ( $\mathrm{mg} / \mathrm{L}$ )

Phosphorus, total (as P)

$\begin{array}{cc} & \\ 0.5 & 3 \\ 91.7 & - \\ 19.3 & 18.8 \\ 65 & 67 \\ 6.2 & 7.3 \\ 9.6 & 4.4 \\ 0.058 & 0.058\end{array}$

\begin{tabular}{c} 
Aug-17 \\
\hline 0.85 \\
3.5 \\
- \\
17.6 \\
69 \\
7.1 \\
0.5 \\
0.054
\end{tabular}

6
-
10.5
122
6.9
0.1
0.524

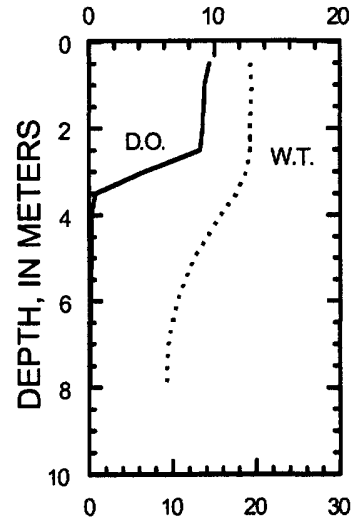

WATER TEMPERATURE (W.T.) IN DEGREES CELSIUS

PH IN STANDARD UNITS

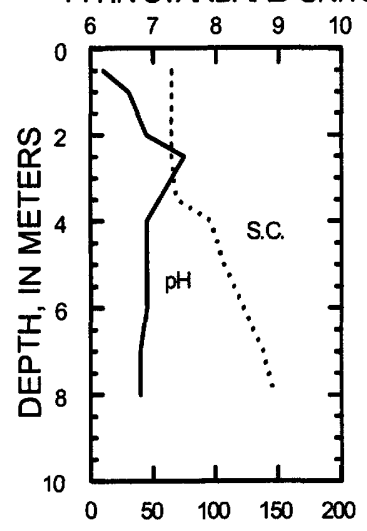

SPECIFIC CONDUCTANCE (S.C.) IN MICROSIEMENS PER CENTIMETER AT 25 DEGREES CELSIUS 


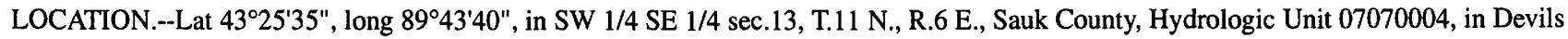
Lake State Park, 3.5 mi south of Baraboo.

DRAINAGE AREA.--4.79 $\mathrm{mi}^{2}$. Area of Devils Lake, 361 acres.

PERIOD OF RECORD.--June 1922 to August 1930, June to August 1932, June 1934 to September 1981, October 1984 to June 1991 (fragmentary), July 1991 to current year. Unpublished daily stage records from October 1981 to September 1984 in District files.

REVISED RECORDS.--WDR WI-78-1: Drainage area.

GAGE.--Water-stage recorder installed July 17, 1991. Datum of gage is $955.00 \mathrm{ft}$, above sea level.

REMARKS.--Lake has no surface outlet. Water removed from lake by pumping or siphon Oct. 1-15 and Sept. 8-30.

EXTREMES FOR PERIOD OF RECORD.--Maximum gage height observed, $14.13 \mathrm{ft}$, July 18, 1993; minimum observed, $1.49 \mathrm{ft}$, Feb. 8, 1965.

EXTREMES FOR CURRENT YEAR.--Maximum recorded gage height, $11.86 \mathrm{ft}$, June 17, 18; minimum recorded, $6.66 \mathrm{ft}$, Nov. 1.

\begin{tabular}{|c|c|c|c|c|c|c|c|c|c|c|c|c|}
\hline \multirow[b]{2}{*}{ DAY } & \multirow[b]{2}{*}{ OCT } & \multirow[b]{2}{*}{ NOV } & \multicolumn{6}{|c|}{$\begin{array}{c}\text { GAGE HEIGHT, FEET } \\
\text { WATER YEAR OCTOBER } 2003 \text { TO SEPTEMBER } 2004 \\
\text { DAILY MEAN VALUES }\end{array}$} & \multirow[b]{2}{*}{ JUN } & \multirow[b]{2}{*}{ JUL } & \multirow[b]{2}{*}{ AUG } & \multirow[b]{2}{*}{ SEP } \\
\hline & & & DEC & JAN & FEB & MAR & APR & MAY & & & & \\
\hline $\begin{array}{l}1 \\
2 \\
3 \\
4 \\
5\end{array}$ & $\begin{array}{l}7.08 \\
7.04 \\
7.00 \\
6.98 \\
6.95\end{array}$ & $\begin{array}{l}6.66 \\
6.67 \\
6.73 \\
6.90 \\
6.90\end{array}$ & $\begin{array}{l}6.95 \\
6.95 \\
6.94 \\
6.94 \\
6.94\end{array}$ & $\begin{array}{l}7.13 \\
7.14 \\
7.15 \\
7.16 \\
7.16\end{array}$ & $\begin{array}{l}7.13 \\
7.14 \\
7.15 \\
7.15 \\
7.15\end{array}$ & $\begin{array}{l}7.27 \\
7.36 \\
7.42 \\
7.47 \\
7.79\end{array}$ & $\begin{array}{l}8.92 \\
8.93 \\
8.93 \\
8.94 \\
8.94\end{array}$ & $\begin{array}{l}8.86 \\
8.84 \\
8.83 \\
8.81 \\
8.79\end{array}$ & $\begin{array}{l}10.67 \\
10.68 \\
10.68 \\
10.67 \\
10.66\end{array}$ & $\begin{array}{l}11.24 \\
11.24 \\
11.21 \\
11.31 \\
11.32\end{array}$ & $\begin{array}{l}10.77 \\
10.77 \\
10.79 \\
10.89 \\
10.86\end{array}$ & $\begin{array}{r}10.10 \\
10.05 \\
10.00 \\
9.98 \\
9.96\end{array}$ \\
\hline $\begin{array}{r}6 \\
7 \\
8 \\
9 \\
10\end{array}$ & $\begin{array}{l}6.94 \\
6.90 \\
6.87 \\
6.86 \\
6.86\end{array}$ & $\begin{array}{l}6.89 \\
6.88 \\
6.86 \\
6.85 \\
6.84\end{array}$ & $\begin{array}{l}6.94 \\
6.93 \\
6.93 \\
6.92 \\
7.00\end{array}$ & $\begin{array}{l}7.17 \\
7.16 \\
7.16 \\
7.16 \\
7.15\end{array}$ & $\begin{array}{l}7.19 \\
7.19 \\
7.19 \\
7.19 \\
7.18\end{array}$ & $\begin{array}{l}8.00 \\
8.08 \\
8.13 \\
8.17 \\
8.20\end{array}$ & $\begin{array}{l}8.94 \\
8.95 \\
8.93 \\
8.93 \\
8.92\end{array}$ & $\begin{array}{l}8.78 \\
8.76 \\
8.79 \\
8.91 \\
8.94\end{array}$ & $\begin{array}{l}10.66 \\
10.66 \\
10.64 \\
10.68 \\
11.17\end{array}$ & $\begin{array}{l}11.31 \\
11.27 \\
11.22 \\
11.19 \\
11.17\end{array}$ & $\begin{array}{l}10.83 \\
10.80 \\
10.77 \\
10.74 \\
10.70\end{array}$ & $\begin{array}{l}9.93 \\
9.89 \\
9.83 \\
9.78 \\
9.73\end{array}$ \\
\hline $\begin{array}{l}11 \\
12 \\
13 \\
14 \\
15\end{array}$ & $\begin{array}{l}6.85 \\
6.85 \\
6.84 \\
6.86 \\
6.84\end{array}$ & $\begin{array}{l}6.83 \\
6.83 \\
6.81 \\
6.80 \\
6.80\end{array}$ & $\begin{array}{l}7.02 \\
7.02 \\
7.01 \\
7.01 \\
7.01\end{array}$ & $\begin{array}{l}7.15 \\
7.15 \\
7.15 \\
7.15 \\
7.15\end{array}$ & $\begin{array}{l}7.18 \\
7.18 \\
7.18 \\
7.17 \\
7.17\end{array}$ & $\begin{array}{l}8.25 \\
8.27 \\
8.28 \\
8.32 \\
8.34\end{array}$ & $\begin{array}{l}8.90 \\
8.89 \\
8.88 \\
8.86 \\
8.85\end{array}$ & $\begin{array}{l}8.96 \\
8.98 \\
9.04 \\
9.12 \\
9.14\end{array}$ & $\begin{array}{l}11.50 \\
11.65 \\
11.72 \\
11.76 \\
11.76\end{array}$ & $\begin{array}{l}11.13 \\
11.09 \\
11.04 \\
10.98 \\
10.93\end{array}$ & $\begin{array}{l}10.66 \\
10.62 \\
10.59 \\
10.56 \\
10.54\end{array}$ & $\begin{array}{l}9.68 \\
9.63 \\
9.59 \\
9.53 \\
9.52\end{array}$ \\
\hline $\begin{array}{l}16 \\
17 \\
18 \\
19 \\
20\end{array}$ & $\begin{array}{l}6.83 \\
6.82 \\
6.80 \\
6.79 \\
6.78\end{array}$ & $\begin{array}{l}6.79 \\
6.80 \\
6.81 \\
6.81 \\
6.80\end{array}$ & $\begin{array}{l}7.02 \\
7.02 \\
7.02 \\
7.01 \\
7.01\end{array}$ & $\begin{array}{l}7.14 \\
7.15 \\
7.15 \\
7.14 \\
7.14\end{array}$ & $\begin{array}{l}7.17 \\
7.16 \\
7.16 \\
7.16 \\
7.18\end{array}$ & $\begin{array}{l}8.35 \\
8.37 \\
8.38 \\
8.39 \\
8.41\end{array}$ & $\begin{array}{l}8.84 \\
8.85 \\
8.92 \\
8.92 \\
8.92\end{array}$ & $\begin{array}{l}9.15 \\
9.16 \\
9.19 \\
9.19 \\
9.21\end{array}$ & $\begin{array}{l}11.77 \\
11.86 \\
11.86 \\
11.81 \\
11.76\end{array}$ & $\begin{array}{l}10.98 \\
11.11 \\
11.10 \\
11.08 \\
11.06\end{array}$ & $\begin{array}{l}10.51 \\
10.49 \\
10.47 \\
10.44 \\
10.38\end{array}$ & $\begin{array}{l}9.50 \\
9.45 \\
9.40 \\
9.34 \\
9.28\end{array}$ \\
\hline $\begin{array}{l}21 \\
22 \\
23 \\
24 \\
25\end{array}$ & $\begin{array}{l}6.77 \\
6.76 \\
6.75 \\
6.74 \\
6.73\end{array}$ & $\begin{array}{l}6.78 \\
6.78 \\
6.96 \\
7.00 \\
7.00\end{array}$ & $\begin{array}{l}7.00 \\
7.00 \\
7.00 \\
6.99 \\
6.99\end{array}$ & $\begin{array}{l}7.13 \\
7.12 \\
7.13 \\
7.13 \\
7.13\end{array}$ & $\begin{array}{l}7.20 \\
7.20 \\
7.21 \\
7.21 \\
7.21\end{array}$ & $\begin{array}{l}8.42 \\
8.43 \\
8.43 \\
8.46 \\
8.50\end{array}$ & $\begin{array}{l}8.94 \\
8.94 \\
8.93 \\
8.92 \\
8.93\end{array}$ & $\begin{array}{r}9.30 \\
9.81 \\
10.11 \\
10.28 \\
10.34\end{array}$ & $\begin{array}{l}11.71 \\
11.68 \\
11.63 \\
11.61 \\
11.56\end{array}$ & $\begin{array}{l}11.07 \\
11.06 \\
11.03 \\
11.00 \\
10.97\end{array}$ & $\begin{array}{l}10.32 \\
10.26 \\
10.23 \\
10.22 \\
10.25\end{array}$ & $\begin{array}{l}9.22 \\
9.18 \\
9.13 \\
9.08 \\
9.03\end{array}$ \\
\hline $\begin{array}{l}26 \\
27 \\
28 \\
29 \\
30 \\
31\end{array}$ & $\begin{array}{l}6.71 \\
6.70 \\
6.69 \\
6.68 \\
6.68 \\
6.67\end{array}$ & $\begin{array}{r}6.99 \\
6.99 \\
6.98 \\
6.98 \\
6.97 \\
---\end{array}$ & $\begin{array}{l}6.99 \\
6.99 \\
7.07 \\
7.11 \\
7.12 \\
7.13\end{array}$ & $\begin{array}{l}7.14 \\
7.15 \\
7.14 \\
7.14 \\
7.14 \\
7.13\end{array}$ & $\begin{array}{r}7.21 \\
7.21 \\
7.20 \\
7.21 \\
\ldots . . \\
\ldots .\end{array}$ & $\begin{array}{l}8.64 \\
8.69 \\
8.75 \\
8.82 \\
8.87 \\
8.90\end{array}$ & $\begin{array}{r}8.92 \\
8.91 \\
8.90 \\
8.89 \\
8.87 \\
. .\end{array}$ & $\begin{array}{l}10.35 \\
10.36 \\
10.35 \\
10.39 \\
10.47 \\
10.62\end{array}$ & $\begin{array}{r}11.50 \\
11.45 \\
11.40 \\
11.35 \\
11.29 \\
\ldots\end{array}$ & $\begin{array}{l}10.94 \\
10.92 \\
10.89 \\
10.85 \\
10.83 \\
10.80\end{array}$ & $\begin{array}{l}10.20 \\
10.32 \\
10.28 \\
10.24 \\
10.19 \\
10.14\end{array}$ & $\begin{array}{r}8.98 \\
8.94 \\
8.88 \\
8.83 \\
8.79 \\
-. .\end{array}$ \\
\hline $\begin{array}{l}\text { MEAN } \\
\text { MAX } \\
\text { MIN }\end{array}$ & $\begin{array}{l}6.83 \\
7.08 \\
6.67\end{array}$ & $\begin{array}{l}6.86 \\
7.00 \\
6.66\end{array}$ & $\begin{array}{l}7.00 \\
7.13 \\
6.92\end{array}$ & $\begin{array}{l}7.14 \\
7.17 \\
7.12\end{array}$ & $\begin{array}{l}7.18 \\
7.21 \\
7.13\end{array}$ & $\begin{array}{l}8.26 \\
8.90 \\
7.27\end{array}$ & $\begin{array}{l}8.91 \\
8.95 \\
8.84\end{array}$ & $\begin{array}{r}9.41 \\
10.62 \\
8.76\end{array}$ & $\begin{array}{l}11.33 \\
11.86 \\
10.64\end{array}$ & $\begin{array}{l}11.08 \\
11.32 \\
10.80\end{array}$ & $\begin{array}{l}10.51 \\
10.89 \\
10.14\end{array}$ & $\begin{array}{r}9.47 \\
10.10 \\
8.79\end{array}$ \\
\hline
\end{tabular}

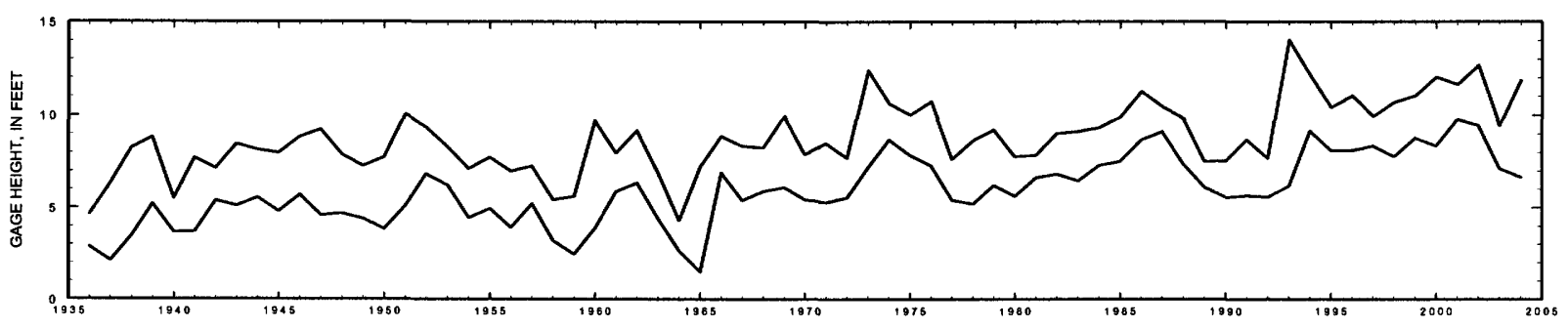




\section{FISH LAKE NEAR SAUK CITY, WI}

LOCATION.--Lat $43^{\circ} 17^{\prime} 27^{\prime \prime}$, long 89 $39^{\prime} 09^{\prime \prime}$ in NE 1/4 SW 1/4 sec.3, T.9 N., R.7 E., Dane County, Hydrologic Unit 07070005, on north side of lake, $0.4 \mathrm{mi}$ southwest of Crystal Lake, and $3.1 \mathrm{mi}$ east of Sauk City.

DRAINAGE AREA.--2.23 $\mathrm{mi}^{2}$. Area of Fish Lake, 252 acres.

PERIOD OF RECORD.--November 1966 to September 1981, April 1985 to May 1987, May 1988, April 1989 to October 1990 (fragmentary); continuous record from October 1990 to November 1996; nonrecording gage November 1996 to current year.

REVISED RECORDS.--WDR WI-92-1: Drainage area. WDR WI-87-1: All published values for the 1987 water year are invalid. Two valid values for water years 1987 and 1988 are available: May 7, 1987, water surface $10.52 \mathrm{ft}$, and May 16, 1988, water surface $10.83 \mathrm{ft}$.

GAGE.--Nonrecording gage. Datum of gage is $848.07 \mathrm{ft}$ above sea level. Local observer, Richard Lillie, reads staff gage when lake is ice-free. REMARKS.--Lake has no surface outlet.

EXTREMES FOR PERIOD OF RECORD.--Maximum gage height observed, $13.39 \mathrm{ft}$, June 14 and 26, 2002; minimum observed, $3.02 \mathrm{ft}$, Aug. 29, 1970.

EXTREMES FOR CURRENT YEAR.--Maximum gage height observed, $12.07 \mathrm{ft}$, Mar. 23; minimum observed, $11.33 \mathrm{ft}$, Oct. 30.

\begin{tabular}{|c|c|c|c|c|c|c|c|c|c|c|c|c|}
\hline \multirow[b]{2}{*}{ DAY } & \multicolumn{8}{|c|}{$\begin{array}{c}\text { GAGE HEIGHT, FEET } \\
\text { WATER YEAR OCTOBER } 2003 \text { TO MARCH } 2004 \\
\text { DAILY MEAN VALUES }\end{array}$} & \multirow[b]{2}{*}{ JUN } & \multirow[b]{2}{*}{ JUL } & \multirow[b]{2}{*}{$A \cup G$} & \multirow[b]{2}{*}{ SEP } \\
\hline & OCT & NOV & DEC & JAN & FEB & MAR & APR & MAY & & & & \\
\hline 1 & -.- & -.- & $\ldots$ & --. & ... & ..- & ... & -.. & ..- & --. & ... & -.. \\
\hline 2 & 11.50 & 11.36 & 11.77 & -.- & --. & $\ldots$ & $\ldots$ & $\ldots$ & $\ldots$ & ..- & ... & $\ldots$ \\
\hline 3 & 11.48 & 11.46 & ... & --. & -.. & -..- & -.. & ... & ... & -. & -.- & -.. \\
\hline 4 & --. & 11.78 & -.. & -.- & --- & --- & -.- & --. & -.- & -- & --. & -.- \\
\hline 5 & 11.47 & 11.78 & --- & -.. & -.. & ... & $\ldots$ & ... & ..- & -.. & ... & ... \\
\hline 6 & --. & ... & -.. & --. & $\ldots$ & -.- & ... & ... & -.- & ... & ... & -.- \\
\hline 7 & -.- & --. & $\ldots$ & -.- & $\ldots$ & --. & ... & ... & -.- & -.. & -.. & -.- \\
\hline 8 & 11.45 & 11.75 & -..- & --. & $\ldots$ & -.- & -.. & --- & -.. & -.. & --. & -.- \\
\hline 9 & -.- & -- & ... & ... & ... & $\ldots$ & $\ldots$ & --- & -.- & --. & --. & -.- \\
\hline 10 & --- & --- & -.. & --. & $\ldots$ & $\ldots$ & ..- & --- & --- & -- & -. & -- \\
\hline 11 & 11.44 & -.. & -.- & $\ldots$ & -.- & -.. & -.- & --- & $\ldots$ & $\ldots$ & -.- & --- \\
\hline 12 & 11.43 & 11.71 & -.. & ... & $\ldots$ & --- & ... & ... & ..- & -.- & -.. & -.. \\
\hline 13 & -.. & $\ldots$ & --- & --- & --- & --. & -.. & ... & --- & --- & -- & --. \\
\hline 14 & 11.48 & -.. & $\cdots$ & -.. & -.. & --. & -.- & -.. & -.- & -.. & -.. & ... \\
\hline 15 & --. & 11.69 & --- & -. & --- & --- & -- & -.- & --- & --- & -- & -- \\
\hline 16 & ... & --- & -.. & --- & $\ldots$ & -.. & -- & --. & --- & -.. & -. & -- \\
\hline 17 & --- & $\ldots$ & --- & -.. & ... & -.. & $\ldots$ & ... & --. & --. & --. & -.. \\
\hline 18 & -- & 11.71 & -- & -- & --. & -.- & --- & --. & -- & $\cdots$ & --- & -- \\
\hline 19 & 11.40 & -.- & --. & --- & -.. & -.. & ... & --- & --- & -.. & -.- & -- \\
\hline 20 & -- & -.- & -- & $\cdots$ & -- & --- & -- & $-\cdots$ & --- & --. & $\cdots$ & $\cdots$ \\
\hline 21 & 11.39 & -.. & --. & ... & -.. & -.. & $\ldots$ & -.- & -.- & -.. & -.. & -. \\
\hline 22 & $\ldots$ & 11.68 & --. & ... & --- & ... & ... & --- & -.. & ... & --. & -.- \\
\hline 23 & 11.38 & 11.80 & $\ldots$ & -..- & --. & 12.07 & -.. & ..- & ... & $\ldots$ & $\ldots$ & ... \\
\hline 24 & -.- & $\ldots$ & -.- & --- & --- & -.- & -.. & ..- & -.. & ... & $\ldots$ & -.. \\
\hline 25 & 11.37 & ... & --. & ..- & -.. & -.- & $\ldots$ & --. & --. & -.. & --. & -- \\
\hline 26 & --- & --- & ... & -.. & -.- & ... & -.. & -.. & --- & --- & -.. & -.. \\
\hline 27 & -- & --. & $\cdots$ & --- & - & -.. & $\cdots$ & --. & -- & -.- & --. & --- \\
\hline 28 & --- & ... & ... & -.. & -.- & -.- & --. & ... & -.- & $\ldots$ & $\ldots$ & -.- \\
\hline 29 & 11.35 & --- & $\cdots$ & -- & -.. & --- & -- & $\cdots$ & --- & --- & --- & - \\
\hline 30 & 11.33 & 11.80 & ... & $\ldots$ & -.. & --. & -.. & ... & -.- & $\ldots$ & -.. & --. \\
\hline 31 & --- & $\ldots$ & $\cdots$ & --- & $\cdots$ & -- & $\cdots$ & $\ldots$ & --- & --- & $\cdots$ & - \\
\hline MEAN & $\ldots$ & --. & -.. & -.- & --. & --- & --. & --- & --. & ... & --- & --- \\
\hline MAX & --. & --- & --- & --- & -.. & -.. & --. & --- & ... & --- & -.. & -.- \\
\hline MIN & --- & --- & $\cdots$ & --- & $\cdots$ & -.- & -- & --- & --. & --- & $\cdots$ & -.- \\
\hline
\end{tabular}

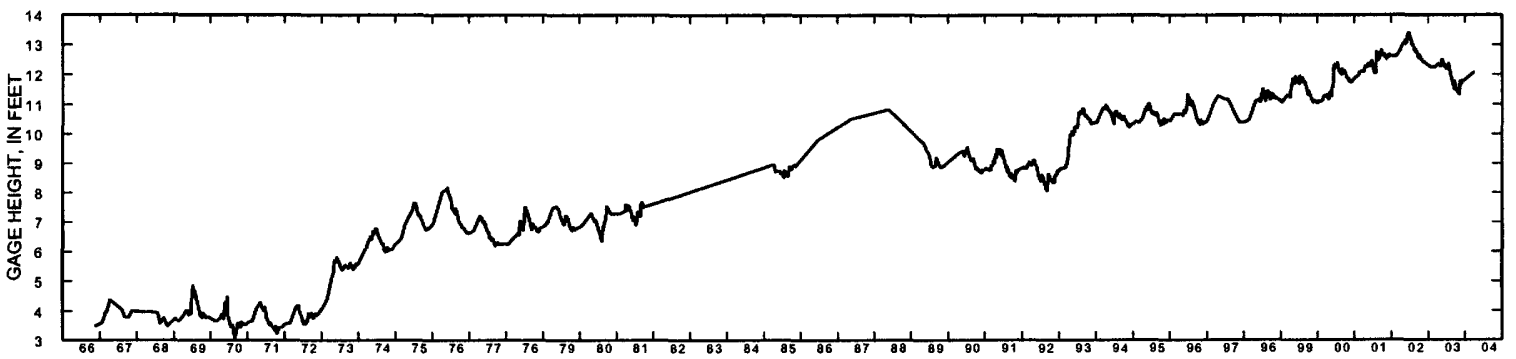




\section{FOREST LAKE NEAR DUNDEE, WI}

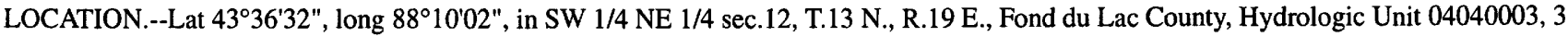
mi south of Dundee.

PERIOD OF RECORD.--March 1994 to August 1996, May to August 2004.

REMARKS.--Lake sampled near center at the deep hole. Lake ice-covered during February sampling. Water-quality analyses done by Wisconsin State Laboratory of Hygiene.

WATER-QUALITY DATA, MAY 3 TO AUGUST 18, 2004
(Milligrams per liter unless otherwise indicated)

Date

Secchi depth $(m)$

Depth of sample $(m)$

Chlorophyll a, phytoplankton $(\mu \mathrm{g} / \mathrm{L})$

Water temperature $\left({ }^{\circ} \mathrm{C}\right)$

Specific conductance $(\mu \mathrm{S} / \mathrm{cm})$

$\mathrm{pH}$

Dissolved oxygen (mg/L)

Phosphorus, total (as P)

Phosphorus, ortho, dissolved (as P)

Nitrogen, $\mathrm{NO}_{2}+\mathrm{NO}_{3}$, diss. (as $\mathrm{N}$ )

Nitrogen, ammonia, dissolved (as $\mathrm{N}$ )

Nitrogen, amm. + org., total (as N)

Color (Pt-Co. scale)

Turbidity (NTU)

Hardness, as $\mathrm{CaCO}_{3}$

Calcium, dissolved (Ca)

Magnesium, dissolved (Mg)

Sodium, dissolved ( $\mathrm{Na}$ )

Potassium, dissolved (K)

Alkalinity, as $\mathrm{CaCO}_{3}$

Sulfate, dissolved $\left(\mathrm{SO}_{4}\right)$

Chloride, dissolved $(\mathrm{Cl})$

Silica, dissolved $\left(\mathrm{SiO}_{2}\right)$

Solids, dissolved, at $180^{\circ} \mathrm{C}$

Iron, dissolved ( $\mathrm{Fe}$ ) $(\mu \mathrm{g} / \mathrm{L})$

Manganese, dissolved, $(\mathrm{Mn})(\mu \mathrm{g} / \mathrm{L})$

\begin{tabular}{|c|c|c|c|c|c|c|c|}
\hline \multicolumn{2}{|c|}{$\frac{\text { May-3 }}{1.9}$} & \multicolumn{2}{|c|}{$\frac{\text { Jun-9 }}{50}$} & \multicolumn{2}{|c|}{$\frac{\text { Jul-14 }}{3.3}$} & \multicolumn{2}{|c|}{ Aug-18 } \\
\hline 0.5 & 8.5 & 0.5 & 9 & 0.5 & 9 & 0.5 & 8.5 \\
\hline 10.6 & - & 4.6 & - & 3.4 & -- & 4.1 & -- \\
\hline 12.6 & 8.1 & 25.1 & 9.7 & 23.9 & 10.4 & 21.2 & 12.1 \\
\hline 249 & 259 & 230 & 247 & 212 & 271 & 200 & 259 \\
\hline 8.4 & 7.7 & 8.3 & 7.1 & 8.4 & 6.9 & 8.6 & 6.8 \\
\hline 11.4 & 3.0 & 11.4 & 0.5 & 12.6 & 0.3 & 11.6 & 0.2 \\
\hline 0.020 & 0.041 & 0.018 & 0.082 & 0.016 & 0.051 & 0.019 & 0.062 \\
\hline 0.003 & - & .- & - & -- & -. & .- & -- \\
\hline$<0.019$ & _- & .- & - & -- & -- & -. & - \\
\hline$<0.015$ & - & -- & - & -- & -- & -- & -- \\
\hline 0.81 & -- & -- & - & -- & -- & -- & -- \\
\hline 5 & - & -- & - & - & - & .- & -- \\
\hline 1.9 & -- & -- & - & -- & - & -- & -- \\
\hline 116 & - & -- & -- & - & -- & -- & -- \\
\hline 26.6 & - & -- & -. & -- & - & -- & - \\
\hline 14.5 & - & -- & .. & - & -- & - & -- \\
\hline 2.5 & - & - & .. & - & -- & - & -- \\
\hline$<1$ & _- & -- & -- & -- & -- & -- & -- \\
\hline 116 & - & - & -- & -- & - & -. & - \\
\hline$<4.5$ & - & -- & -- & - & - & -. & - \\
\hline 4.9 & - & - & -- & -- & - & -- & -- \\
\hline 0.126 & -- & -- & -- & -- & -- & -- & -- \\
\hline 128 & -- & -- & .- & -- & -- & - & - \\
\hline$<100$ & -- & -- & - & -- & - & -- & -- \\
\hline 1.0 & - & -- & -- & -- & -- & -- & - \\
\hline
\end{tabular}

5-3-04
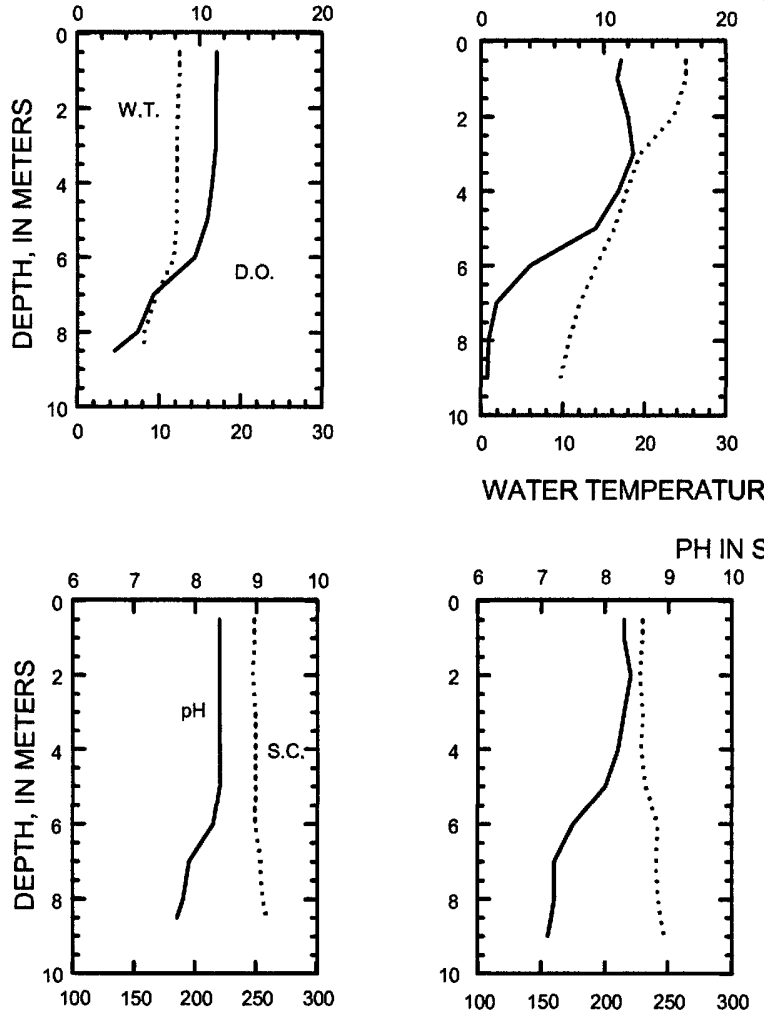

7-14-04

8-18-04
DISSOLVED OXYGEN (D.O.) IN MILLIGRAMS PER LITER
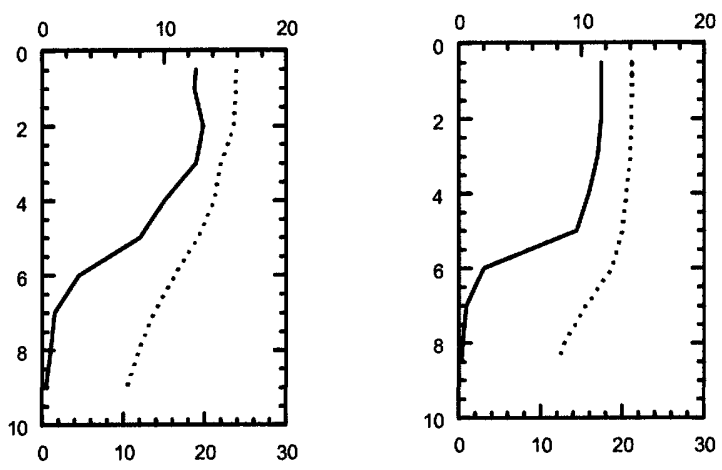

WATER TEMPERATURE (W.T.) IN DEGREES CELSIUS

PH IN STANDARD UNITS

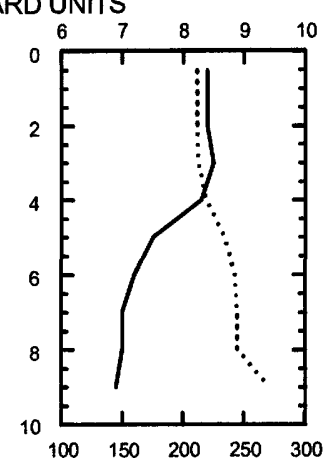

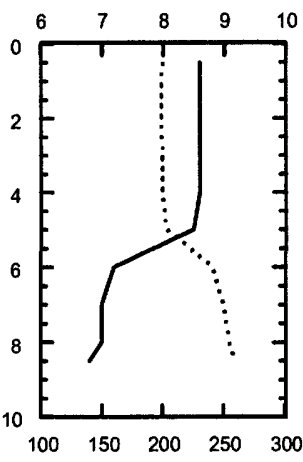

SPECIFIC CONDUCTANCE (S.C.) IN MICROSIEMENS PER CENTIMETER AT 25 DEGREES CELSIUS 

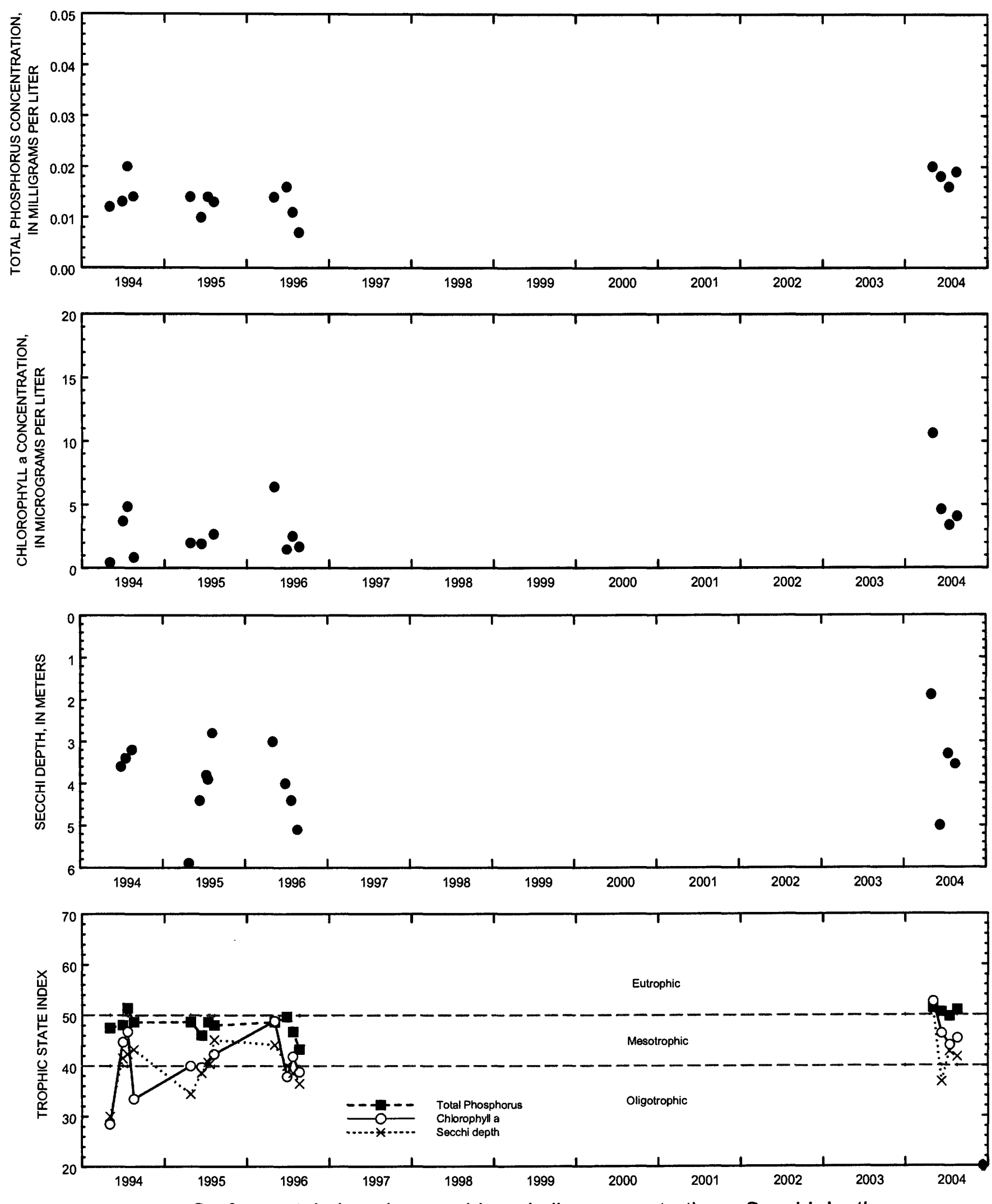

Surface total phosphorus, chlorophyll a concentrations, Secchi depths, and TSI data for Forest Lake near Dundee, Wisconsin. 


\section{GENEVA LAKE AT LAKE GENEVA, WI}

LOCATION.--Lat $42^{\circ} 35^{\prime} 25^{\prime \prime}$, long $88^{\circ} 26^{\prime} 04^{\prime \prime}$ in SE 1/4 NW 1/4 sec.36, T.2 N., R.17 E., Walworth County, Hydrologic Unit 07120006, at Geneva Lake dam at Center Street at Lake Geneva.

DRAINAGE AREA.--28.7 $\mathrm{mi}^{2}$. Area of Geneva Lake, 5,262 acres.

PERIOD OF RECORD.--October 1997 to August 2002, December 2002 to current year.

GAGE.--Water-stage recorder. Datum of gage is $862.08 \mathrm{ft}$ above sea level. Intermittent staff-gage readings January to February.

REMARKS.--Gage-height telemeter at station.

EXTREMES FOR PERIOD OF RECORD.--Maximum gage height, 3.29 ft, June 13, 2000; minimum gage height, $1.50 \mathrm{ft}$, Oct. 11,2003 (affected by wind).

EXTREMES FOR CURRENT YEAR.--Maximum gage height, $3.28 \mathrm{ft}$, May 28; minimum gage height, $1.50 \mathrm{ft}$, Oct. 11 (affected by wind).

\begin{tabular}{|c|c|c|c|c|c|c|c|c|c|c|c|c|}
\hline \multirow[b]{2}{*}{ DAY } & \multicolumn{9}{|c|}{$\begin{array}{c}\text { GAGE HEIGHT, FEET } \\
\text { WATER YEAR OCTOBER } 2003 \text { TO SEPTEMBER } 2004 \\
\text { DAILY MEAN VALUES }\end{array}$} & \multirow[b]{2}{*}{ JUL } & \multirow[b]{2}{*}{$A \cup G$} & \multirow[b]{2}{*}{ SEP } \\
\hline & OCT & NOV & DEC & JAN & FEB & MAR & APR & MAY & JUN & & & \\
\hline 1 & 1.75 & 1.67 & 1.99 & 2.19 & -..- & 2.46 & 2.56 & 2.51 & 2.92 & 2.59 & 2.36 & 2.27 \\
\hline 2 & 1.73 & 1.77 & 1.97 & 2.20 & -.. & 2.48 & 2.55 & 2.50 & 2.87 & 2.56 & 2.35 & 2.27 \\
\hline 3 & 1.74 & 1.87 & 1.96 & 2.21 & $\ldots$ & 2.48 & 2.54 & 2.49 & 2.83 & 2.56 & 2.34 & 2.27 \\
\hline 4 & 1.71 & 1.96 & 1.95 & 2.20 & e2.27 & 2.49 & 2.53 & 2.50 & 2.80 & 2.62 & 2.39 & 2.27 \\
\hline 5 & 1.70 & 2.02 & 1.97 & 2.22 & $\ldots$ & 2.62 & 2.52 & 2.48 & 2.77 & 2.59 & 2.37 & 2.28 \\
\hline 6 & 1.69 & 2.02 & 1.97 & $\ldots$ & --. & 2.60 & 2.52 & 2.48 & 2.75 & 2.60 & 2.36 & 2.29 \\
\hline 7 & 1.69 & 2.01 & 1.97 & --. & -.- & 2.59 & 2.51 & 2.45 & 2.74 & 2.58 & 2.35 & 2.26 \\
\hline 8 & 1.70 & 1.98 & 1.97 & --- & --. & 2.56 & 2.51 & 2.48 & 2.73 & 2.55 & 2.34 & 2.23 \\
\hline 9 & 1.69 & 1.98 & 1.98 & -.. & -.- & 2.53 & 2.50 & 2.50 & 2.71 & 2.53 & 2.34 & 2.22 \\
\hline 10 & 1.69 & 1.98 & 2.15 & --- & $\cdots$ & 2.51 & 2.49 & 2.51 & 2.71 & 2.54 & 2.32 & 2.22 \\
\hline 11 & 1.70 & 1.98 & 2.19 & .-. & ... & 2.50 & 2.48 & 2.51 & 2.74 & 2.54 & 2.29 & 2.21 \\
\hline 12 & 1.71 & 2.01 & 2.18 & ... & -.. & 2.47 & 2.47 & 2.54 & 2.78 & 2.54 & 2.26 & 2.21 \\
\hline 13 & 1.70 & 1.97 & 2.17 & $\ldots$ & -..- & 2.44 & 2.46 & 2.71 & 2.78 & 2.54 & 2.24 & 2.21 \\
\hline 14 & 1.74 & 1.96 & 2.18 & --- & --. & 2.46 & 2.46 & 2.79 & 2.76 & 2.52 & 2.23 & 2.20 \\
\hline 15 & 1.74 & 1.94 & 2.19 & --. & --. & 2.42 & 2.46 & 2.81 & 2.74 & 2.51 & 2.23 & 2.22 \\
\hline 16 & 1.72 & 1.94 & 2.21 & -.- & -.-. & 2.40 & 2.46 & 2.79 & 2.71 & 2.50 & 2.23 & 2.20 \\
\hline 17 & 1.71 & 1.94 & 2.19 & $\ldots$ & -.- & 2.40 & 2.47 & 2.78 & 2.75 & 2.47 & 2.22 & 2.17 \\
\hline 18 & 1.71 & 2.02 & 2.18 & --- & --. & 2.39 & 2.50 & 2.84 & 2.76 & 2.47 & 2.23 & 2.16 \\
\hline 19 & 1.69 & 2.05 & 2.18 & -.. & $\ldots$ & 2.38 & 2.51 & 2.81 & 2.71 & 2.47 & 2.21 & 2.16 \\
\hline 20 & 1.71 & 2.05 & 2.18 & --. & --- & 2.38 & 2.48 & 2.81 & 2.69 & 2.46 & 2.19 & 2.15 \\
\hline 21 & 1.69 & 2.02 & 2.19 & $\ldots$ & ... & 2.35 & 2.56 & 2.85 & 2.70 & 2.47 & 2.18 & 2.14 \\
\hline 22 & 1.68 & 2.02 & 2.17 & --. & -.- & 2.34 & 2.53 & 3.01 & 2.73 & 2.49 & 2.19 & 2.12 \\
\hline 23 & 1.67 & 2.07 & 2.17 & --- & $\ldots$ & 2.32 & 2.53 & 3.06 & 2.71 & 2.46 & 2.16 & 2.12 \\
\hline 24 & 1.67 & 2.09 & 2.17 & --. & ... & 2.36 & 2.51 & 3.04 & 2.71 & 2.44 & 2.17 & 2.11 \\
\hline 25 & 1.70 & 2.04 & 2.17 & -.. & e2.42 & 2.38 & 2.55 & 2.99 & 2.70 & 2.42 & 2.21 & 2.08 \\
\hline 26 & 1.70 & 2.01 & 2.16 & --. & -.- & 2.48 & 2.55 & 2.95 & 2.68 & 2.41 & 2.22 & 2.08 \\
\hline 27 & 1.68 & 2.01 & 2.16 & --- & 2.42 & 2.49 & 2.53 & 2.91 & 2.65 & 2.40 & 2.24 & 2.07 \\
\hline 28 & 1.70 & 2.02 & 2.20 & $e 2.28$ & 2.42 & 2.53 & 2.54 & 2.85 & 2.64 & 2.39 & 2.25 & 2.04 \\
\hline 29 & 1.67 & 2.02 & 2.21 & -.. & 2.45 & 2.58 & 2.53 & 2.82 & 2.62 & 2.38 & 2.28 & 2.03 \\
\hline 30 & 1.67 & 2.00 & 2.21 & -.- & ... & 2.57 & 2.50 & 2.87 & 2.61 & 2.37 & 2.28 & 2.02 \\
\hline 31 & 1.68 & --. & 2.21 & --- & -.. & 2.57 & ... & 2.95 & --. & 2.36 & 2.27 & - \\
\hline MEAN & 1.70 & 1.98 & 2.12 & ... & $\cdots$ & 2.47 & 2.51 & 2.73 & 2.73 & 2.49 & 2.27 & 2.18 \\
\hline MAX & 1.75 & 2.09 & 2.21 & -.- & -.. & 2.62 & 2.56 & 3.06 & 2.92 & 2.62 & 2.39 & 2.29 \\
\hline MIN & 1.67 & 1.67 & 1.95 & .-. & ... & 2.32 & 2.46 & 2.45 & 2.61 & 2.36 & 2.16 & 2.02 \\
\hline
\end{tabular}

e Estimated

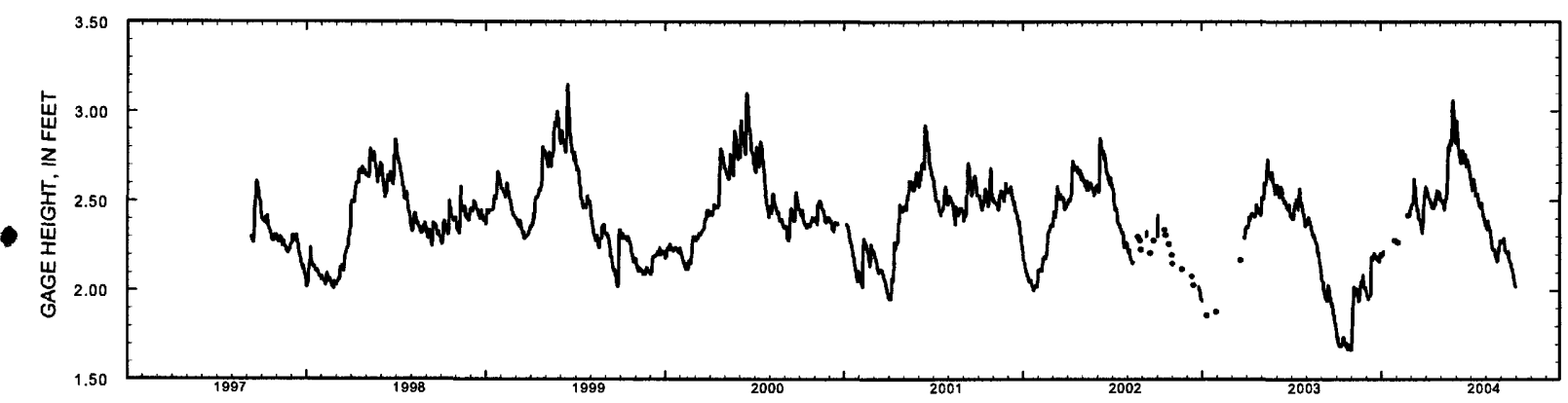




\section{GENEVA LAKE AT WEST END NEAR WILLIAMS BAY, WI}

LOCATION.--Lat 42³3'29", long 88³2'33", in NE 1/4 SE 1/4, sec.12, T.1 N., R.16 E., Walworth County, Hydrologic Unit 07120006, 1.3 mi south of Williams Bay.

DRAINAGE AREA.--28.7 $\mathrm{mi}^{2}$.

PERIOD OF RECORD.--April 1997 to current year.

REMARKS.--Lake sampled at deep hole at a depth of about $43 \mathrm{~m}$. Water-quality analyses done by Wisconsin State Laboratory of Hygiene. Samples for determination of chlorophyll $a$ concentration are collected from the top $0.5 \mathrm{~m}$ of the lake.

WATER-QUALITY DATA, OCTOBER 24, 2003 TO APRIL 13, 2004 (Milligrams per liter unless otherwise indicated)

Date

Lake stage (ft)

Secchi depth (m)

Depth of sample (m)

Chlorophyll a, phytoplankton ( $\mu \mathrm{g} / \mathrm{L})$

Water temperature $\left({ }^{\circ} \mathrm{C}\right)$

Specific conductance $(\mu \mathrm{S} / \mathrm{cm})$

$\mathrm{pH}$

Dissolved oxygen (mg/L)

Phosphorus, total (as P)

Phosphorus, ortho, dissolved (as P)

Nitrogen, $\mathrm{NO}_{2}+\mathrm{NO}_{3}$, diss. (as $\mathrm{N}$ )

Nitrogen, ammonia, dissolved (as $\mathrm{N}$ )

Nitrogen, amm. + org., total (as N)

Nitrogen, total (as $\mathrm{N}$ )

Color (Pt-Co. scale)

Turbidity (NTU)

Hardness, as $\mathrm{CaCO}_{3}$

Calcium, dissolved $(\mathrm{Ca})$

Magnesium, dissolved (Mg)

Sodium, dissolved ( $\mathrm{Na}$ )

Potassium, dissolved (K)

Alkalinity, as $\mathrm{CaCO}_{3}$

Sulfate, dissolved $\left(\mathrm{SO}_{4}\right)$

Chloride, dissolved (Cl)

Silica, dissolved $\left(\mathrm{SiO}_{2}\right)$

Solids, dissolved, at $180^{\circ} \mathrm{C}$

Iron, dissolved $(\mathrm{Fe})(\mu \mathrm{g} / \mathrm{L})$

Lead, (Pb) ( $\mu \mathrm{g} / \mathrm{L})$

Marigariese, dissolved, (Mn) ( $\mu \mathrm{g} / \mathrm{L})$

\begin{tabular}{|c|c|c|}
\hline & & $\begin{array}{c}\text { Oct-24 } \\
1.67 \\
6.2\end{array}$ \\
\hline 0.5 & 18 & \\
\hline 3.54 & -- & \\
\hline 13.5 & 13.4 & \\
\hline 523 & 523 & \\
\hline 8.1 & 8.1 & \\
\hline 9.6 & 8.1 & \\
\hline 0.013 & 0.013 & \\
\hline 0.003 & - & \\
\hline$<0.022$ & - & \\
\hline$<0.013$ & - & \\
\hline 0.32 & -. & \\
\hline- & - & \\
\hline- & -- & \\
\hline- & - & \\
\hline- & -- & \\
\hline- & - & \\
\hline- & - & \\
\hline- & - & \\
\hline- & - & \\
\hline-- & - & \\
\hline- & - & \\
\hline- & - & \\
\hline- & -- & \\
\hline- & - & \\
\hline - & - & \\
\hline- & - & \\
\hline-- & - & \\
\hline
\end{tabular}

$\frac{\text { Apr-13 }}{2.46}$

5.3

$\begin{array}{cc}0.5 & 43 \\ 3.86 & - \\ 4.9 & 4.4 \\ 525 & 525 \\ 8.2 & 8.2 \\ 13.5 & 13.1 \\ 0.011 & 0.011 \\ 0.002 & 0.002 \\ 0.048 & 0.057 \\ 0.025 & 0.135 \\ 0.38 & 0.38 \\ 0.43 & 0.44 \\ 5 & 5 \\ <1 & <1 \\ 230 & 230 \\ 34.4 & 34.6 \\ 34.5 & 34.7 \\ 18.4 & 18.7 \\ 2 & 2 \\ 184 & 184 \\ 31.1 & 31.6 \\ 37.5 & 37.7 \\ 1.73 & 1.84 \\ 282 & 288 \\ <100 & <100 \\ <1 & <1 \\ <1 & <1\end{array}$

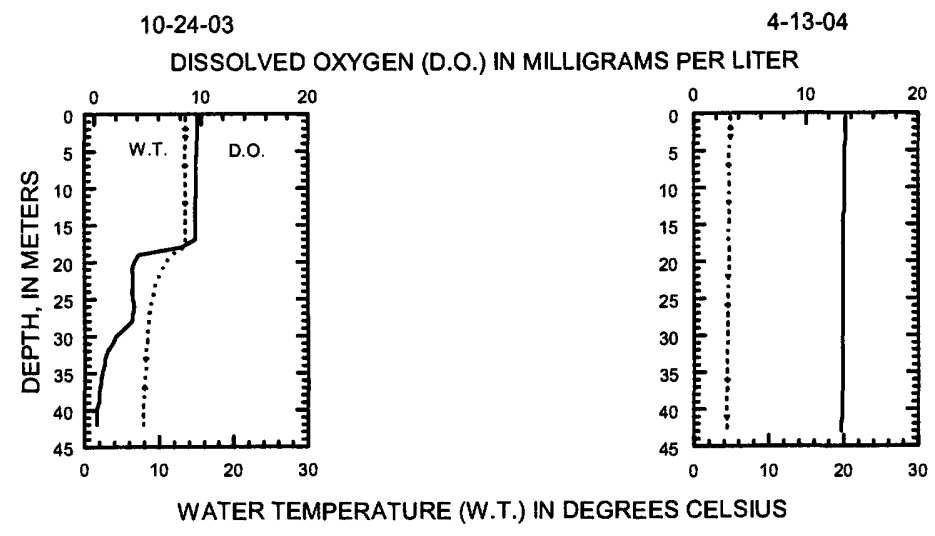

PH IN STANDARD UNITS
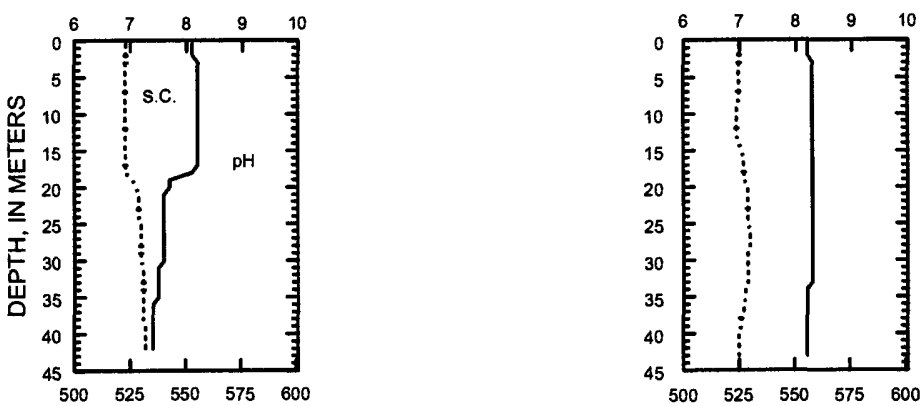

SPECIFIC CONDUCTANCE (S.C.) IN MICROSIEMENS PER CENTIMETER AT 25 DEGREES CELSIUS 
WATER-QUALITY DATA, JUNE 22, 2004

(Milligrams per liter unless otherwise indicated)

Date

Lake stage (ft)

Secchi depth $(m)$

Depth of sample $(\mathrm{m})$

Chlorophyll a, phytoplarikton ( $\mu g / L)$

Water temperature $\left({ }^{\circ} \mathrm{C}\right)$

Specific conductance $(\mu \mathrm{S} / \mathrm{cm})$

$\mathrm{pH}$

Dissolved oxygen (mg/L)

Phosphorus, total (as P)

Phosphorus, ortho, dissolved (as $\mathrm{P}$ )

Nitrogen, $\mathrm{NO}_{2}+\mathrm{NO}_{3}$, diss. (as $\mathrm{N}$ )

Nitrogen, ammoria, dissolved (as $\mathrm{N}$ )

Nitrogen, amm. + org., total (as N)

\begin{tabular}{ccccc} 
& & \multicolumn{3}{c}{$\frac{\text { Jun-22 }}{2.73}$} \\
0.5 & & & 5.3 & \\
4.02 & 8 & 20 & & 33 \\
20 & - & - & - \\
510 & 19.8 & 9.9 & 8.6 \\
8.3 & 511 & 531 & 534 \\
9.7 & 8.3 & 8.7 & 8.4 \\
0.007 & 9.6 & 9.1 & 9.3 \\
$<0.002$ & 0.009 & 0.007 & 0.007 \\
$<0.019$ & -- & - & - \\
$<0.015$ & - & - & - \\
0.3 & -- & - & - \\
& - & - & -
\end{tabular}

6-22-04

DISSOLVED OXYGEN (D.O.) IN MILLIGRAMS PER LITER

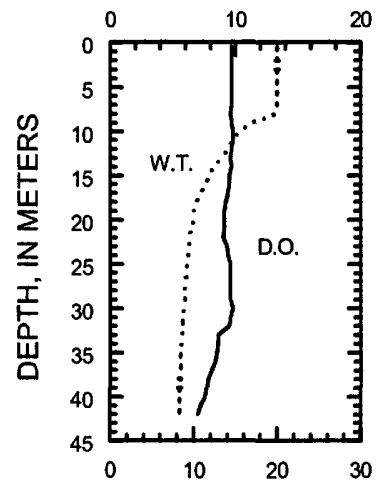

WATER TEMPERATURE (W.T.) IN DEGREES CELSIUS

PH IN STANDARD UNITS

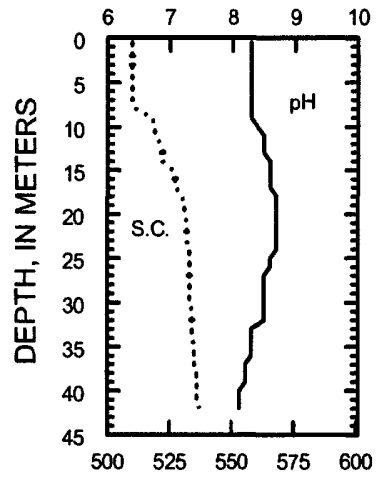

SPECIFIC CONDUCTANCE (S.C.) IN MICROSIEMENS PER CENTIMETER AT 25 DEGREES CELSIUS 
WATER-QUALITY DATA, JULY 20, 2004

(Milligrams per liter unless otherwise indicated)

Date

Lake stage (ft)

Secchi depth (m)

Depth of sample $(m)$

Chlorophyll a, phytoplankton $(\mu \mathrm{g} / \mathrm{L})$

Water temperature $\left({ }^{\circ} \mathrm{C}\right)$

Specific conductance $(\mu \mathrm{S} / \mathrm{cm})$

$\mathrm{pH}$

Dissolved oxygen (mg/L)

Phosphorus, total (as P)

Phosphorus, ortho, dissolved (as P)

Nitrogen, $\mathrm{NO}_{2}+\mathrm{NO}_{3}$, diss. (as $\mathrm{N}$ )

Nitrogen, ammonia, dissolved (as N)

Nitrogen, amm. + org., total (as N)

\begin{tabular}{ccccc} 
& & \multicolumn{3}{c}{$\frac{\text { Jul-20 }}{2.46}$} \\
0.5 & & \multicolumn{3}{c}{5.3} \\
1.6 & - & 25 & & 33 \\
23 & 22.5 & - & - \\
513 & 513 & 536 & 8.5 \\
8.4 & 8.4 & 9.1 & 537 \\
9.2 & 9 & 8.3 & 8.6 \\
0.008 & 0.01 & 0.006 & 7.7 \\
0.002 & - & - & 0.009 \\
$<0.019$ & - & - & - \\
$<0.015$ & - & - & - \\
0.35 & - & - & -
\end{tabular}

7-20-04

DISSOLVED OXYGEN (D.O.) IN MILIGRAMS PER LITER

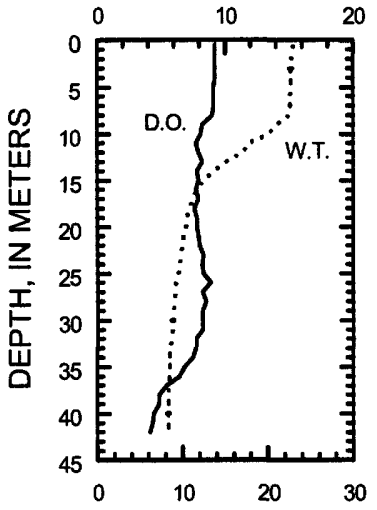

WATER TEMPERATURE (W.T.) IN DEGREES CELSIUS

PH IN STANDARD UNITS

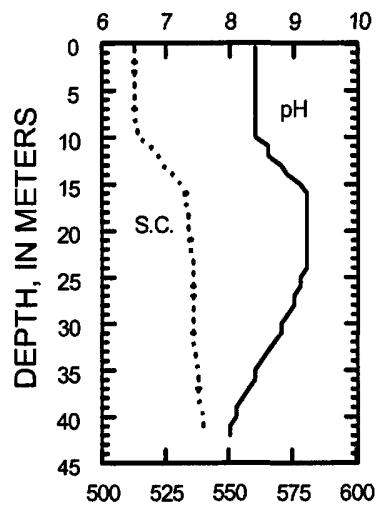

SPECIFIC CONDUCTANCE (S.C.) IN MICROSIEMENS PER CENTIMETER AT 25 DEGREES CELSIUS 
WATER-QUALITY DATA, AUGUST 19, 2004

(Milligrams per liter unless otherwise indicated)

Date

Lake stage (ft)

Secchi depth $(m)$

Depth of sample (m)

Chlorophyll a, phytoplankton $(\mu \mathrm{g} / \mathrm{L})$

Water temperature $\left({ }^{\circ} \mathrm{C}\right)$

Specific conductance $(\mu \mathrm{S} / \mathrm{cm})$

$\mathrm{pH}$

Dissolved oxygen (mg/L)

Phosphorus, total (as P)

Phosphorus, ortho, dissolved (as $\mathrm{P}$ )

Nitrogen, $\mathrm{NO}_{2}+\mathrm{NO}_{3}$, diss. (as $\mathrm{N}$ )

Nitrogen, ammonia, dissolved (as $\mathrm{N}$ )

Nitrogen, amm. + org., total (as N)

\begin{tabular}{ccccc} 
& & \multicolumn{3}{c}{$\frac{\text { Aug-19 }}{2.21}$} \\
0.5 & & & 5.8 & \\
2.65 & 12 & 21 & & 33 \\
20.8 & -- & - & - \\
510 & 20.6 & 10.6 & 8.6 \\
8 & 511 & 532 & 533 \\
8.3 & 8.2 & 8.3 & 8.1 \\
0.009 & 7.8 & 6.2 & 4.9 \\
0.002 & 0.01 & 0.008 & 0.008 \\
$<0.019$ & -- & -- & - \\
0.038 & -- & - & - \\
0.43 & -- & -- & -
\end{tabular}

8-19-04

DISSOLVED OXYGEN (D.O.) IN MULIGRAMS PER UTER

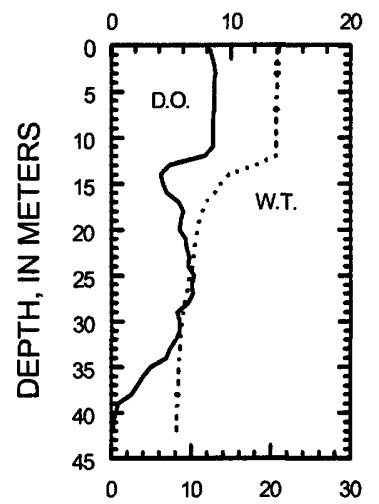

WATER TEMPERATURE (W.T.) IN DEGREES CELSIUS

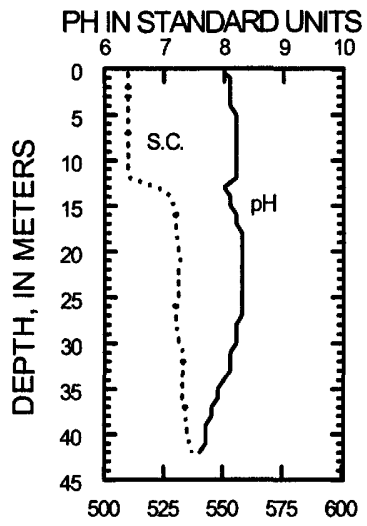

SPECIFIC CONDUCTANCE (S.C.) IN MICROSIEMENS PER CENTIMETER AT 25 DEGREES CELSIUS 
WATER-QUALITY DATA, SEPTEMBER 22, 2004

(Milligrams per liter unless otherwise indicated)

Date

Lake stage (ft)

Secchi depth $(m)$

Depth of sample (m)

Chlorophyll a, phytoplankton $(\mu g / L)$

Water temperature $\left({ }^{\circ} \mathrm{C}\right)$

Specific conductance $(\mu \mathrm{S} / \mathrm{cm})$

$\mathrm{pH}$

Dissolved oxygen (mg/L)

Phosphorus, total (as P)

Phosphorus, ortho, dissolved (as $\mathrm{P}$ )

Nitrogen, $\mathrm{NO}_{2}+\mathrm{NO}_{3}$, diss. (as $\mathrm{N}$ )

Nitrogen, ammonia, dissolved (as $\mathrm{N}$ )

Nitrogen, amm. + org., total (as $\mathrm{N}$ )

Nitrogen, total (as $\mathrm{N}$ )

\begin{tabular}{ccccc} 
& & \multicolumn{3}{c}{$\frac{\text { Sep-22 }}{2.12}$} \\
& & & 5.5 & \\
0.5 & 12 & 25 & & 33 \\
2.61 & -- & -- & -- \\
21 & 20.7 & 9.6 & 8.6 \\
510 & 511 & 529 & 530 \\
8.2 & 8.2 & 8.4 & 8 \\
8.3 & 7 & 5.1 & 2.9 \\
0.011 & 0.013 & 0.008 & 0.015 \\
0.003 & $<0.002$ & 0.003 & 0.008 \\
$<0.019$ & $<0.019$ & 0.123 & 0.263 \\
$<0.015$ & $<0.015$ & $<0.015$ & $<0.015$ \\
0.45 & 0.46 & 0.33 & 0.4 \\
- & - & 0.45 & 0.66
\end{tabular}

\section{9-22-04}

DISSOLVED OXYGEN (D.O.) IN MILLIGRAMS PER LITER

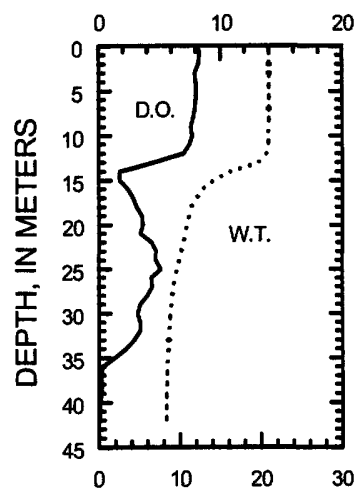

WATER TEMPERATURE (W.T.) IN DEGREES CELSIUS

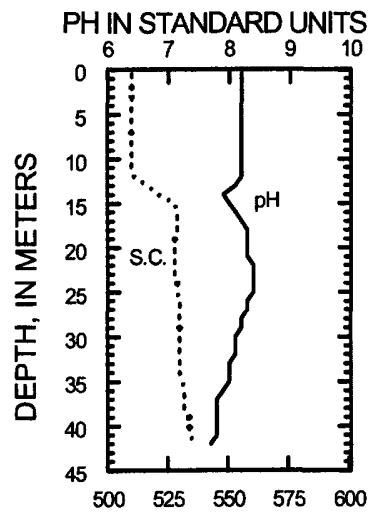

SPECIFIC CONDUCTANCE (S.C.) IN MICROSIEMENS PER CENTIMETER AT 25 DEGREES CELSIUS 

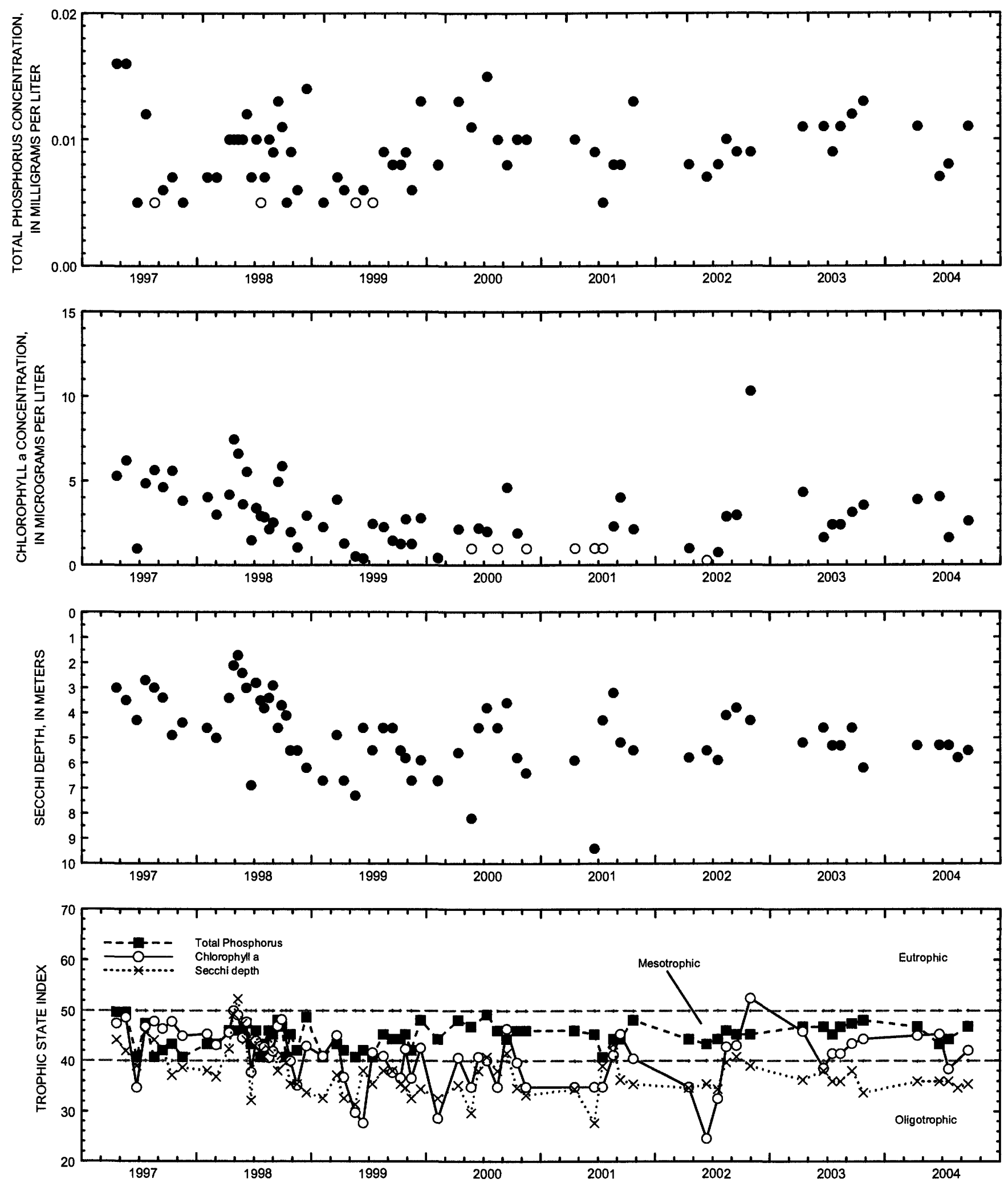

Surface total phosphorus, chlorophyll a concentrations, Secchi depths, and TSI data for Lake Geneva, West End, near Williams Bay, Wisconsin.

(Circles on the first two plots indicate laboratory detection limit for selected analyses. Actual concentrations for these particular analyses are less than the plotted circles.) 


\section{GREEN LAKE AT COUNTY TRUNK HIGHWAY A NEAR GREEN LAKE, WI}

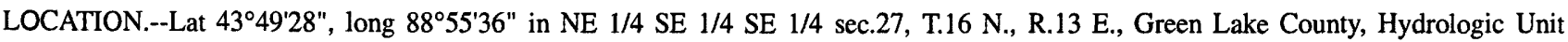
04030201 , on left bank at downstream side of County Trunk Highway A, 2.3 mi southeast of Green Lake.

DRAINAGE AREA.--103 $\mathrm{mi}^{2}$.

PERIOD OF RECORD.--October 1993 to current year.

GAGE.--Water-stage recorder. Datum of gage is $790.00 \mathrm{ft}$ above sea level.

REMARKS.--Lake level regulated by dam at outlet at Green Lake. Gage-height telemeter at station.

EXTREMES FOR PERIOD OF RECORD.--Maximum recorded gage height, $7.64 \mathrm{ft}$, June 17, 2004; minimum recorded, $5.41 \mathrm{ft}$, Jan. 17 , 1995.

EXTREMES FOR CURRENT YEAR.--Maximum recorded gage height, $7.64 \mathrm{ft}$, June 17; minimum recorded, $5.73 \mathrm{ft}$, Oct. 30, Nov. $1,2$.

GAGE HEIGHT, FEET

WATER YEAR OCTOBER 2003 TO SEPTEMBER 2004

DAILY MEAN VALUES

\begin{tabular}{|c|c|c|c|c|c|c|c|c|c|c|c|c|}
\hline DAY & OCT & NOV & DEC & JAN & FEB & MAR & APR & MAY & JUN & JUL & AUG & SEP \\
\hline 1 & 5.91 & 5.75 & 6.30 & 6.37 & 6.32 & 6.42 & 6.60 & 6.56 & 7.50 & 6.91 & 6.31 & 6.27 \\
\hline 2 & 5.88 & 5.77 & 6.27 & 6.38 & 6.32 & 6.54 & 6.59 & 6.55 & 7.50 & 6.84 & 6.33 & 6.27 \\
\hline 3 & 5.90 & 5.78 & 6.27 & 6.40 & 6.35 & 6.60 & 6.58 & 6.54 & 7.49 & 6.79 & 6.32 & 6.27 \\
\hline 4 & 5.89 & 5.92 & 6.27 & 6.37 & 6.34 & 6.65 & 6.57 & 6.54 & 7.49 & 6.82 & 6.32 & 6.26 \\
\hline 5 & 5.87 & 5.96 & 6.27 & 6.39 & 6.34 & 6.81 & 6.55 & 6.52 & 7.41 & 6.79 & 6.31 & 6.25 \\
\hline 6 & 5.87 & 5.95 & 6.27 & 6.40 & 6.37 & 6.95 & 6.54 & 6.51 & 7.37 & 6.81 & 6.29 & 6.26 \\
\hline 7 & 5.87 & 5.95 & 6.27 & 6.36 & 6.37 & 7.03 & 6.53 & - & 7.33 & 6.78 & 6.29 & 6.24 \\
\hline 8 & 5.87 & 5.92 & 6.27 & 6.34 & 6.37 & 7.04 & 6.52 &.- & 7.28 & 6.73 & 6.27 & 6.20 \\
\hline 9 & 5.87 & 5.92 & 6.27 & 6.34 & 6.37 & 7.02 & 6.51 & -- & 7.25 & 6.69 & 6.28 & 6.19 \\
\hline 10 & 5.86 & 5.90 & 6.34 & 6.34 & 6.36 & 7.00 & 6.49 & $-\cdots$ & 7.21 & 6.67 & 6.27 & 6.18 \\
\hline 11 & 5.87 & 5.92 & 6.39 & 6.33 & 6.35 & 6.98 & 6.48 & -- & 7.41 & 6.63 & 6.24 & 6.18 \\
\hline 12 & 5.90 & 5.96 & 6.37 & 6.33 & 6.35 & 6.91 & 6.46 & - & 7.50 & 6.58 & 6.20 & 6.16 \\
\hline 13 & 5.87 & 5.94 & 6.36 & 6.32 & 6.35 & 6.86 & 6.45 & $\cdots$ & 7.57 & 6.53 & 6.18 & 6.15 \\
\hline 14 & 5.89 & 5.91 & 6.36 & 6.32 & 6.34 & 6.84 & 6.44 & $\ldots$ & 7.60 & 6.56 & 6.16 & 6.15 \\
\hline 15 & 5.88 & 5.91 & 6.36 & 6.32 & 6.33 & 6.79 & 6.43 & - & 7.60 & 6.53 & 6.16 & 6.16 \\
\hline 16 & 5.86 & 5.91 & 6.39 & 6.32 & 6.33 & 6.75 & 6.43 & - & 7.58 & 6.52 & 6.15 & 6.16 \\
\hline 17 & 5.85 & 5.91 & 6.38 & 6.33 & 6.33 & 6.72 & 6.43 & --- & 7.60 & 6.50 & 6.19 & 6.13 \\
\hline 18 & 5.84 & 5.99 & 6.37 & 6.33 & 6.32 & 6.69 & 6.49 & --. & 7.59 & 6.49 & 6.22 & 6.12 \\
\hline 19 & 5.83 & 6.02 & 6.37 & 6.33 & 6.31 & 6.67 & 6.53 & --- & 7.54 & 6.48 & 6.22 & 6.11 \\
\hline 20 & 5.84 & 6.02 & 6.36 & 6.32 & 6.33 & 6.66 & 6.53 & 6.92 & 7.51 & 6.46 & 6.20 & 6.09 \\
\hline 21 & 5.82 & 6.01 & 6.36 & 6.32 & 6.35 & 6.62 & 6.57 & 6.98 & 7.50 & 6.47 & 6.18 & 6.07 \\
\hline 22 & 5.81 & 5.99 & 6.35 & 6.31 & 6.35 & 6.60 & 6.57 & 7.10 & 7.41 & 6.46 & 6.17 & 6.06 \\
\hline 23 & 5.80 & 6.19 & 6.35 & 6.31 & 6.37 & 6.58 & 6.57 & 7.22 & 7.35 & 6.43 & 6.13 & 6.05 \\
\hline 24 & 5.79 & 6.28 & 6.35 & 6.32 & 6.37 & 6.57 & 6.57 & 7.36 & 7.35 & 6.40 & 6.13 & 6.04 \\
\hline 25 & 5.80 & 6.25 & 6.34 & 6.32 & 6.36 & 6.57 & 6.58 & 7.42 & 7.27 & 6.36 & 6.14 & 6.01 \\
\hline 26 & 5.79 & 6.26 & 6.33 & 6.33 & 6.36 & 6.58 & 6.59 & 7.43 & 7.19 & 6.35 & 6.15 & 6.00 \\
\hline 27 & 5.77 & 6.27 & 6.33 & 6.33 & 6.35 & 6.58 & 6.58 & 7.43 & 7.13 & 6.34 & 6.27 & 5.99 \\
\hline 28 & 5.78 & 6.29 & 6.38 & 6.33 & 6.35 & 6.60 & 6.57 & 7.38 & 7.09 & 6.33 & 6.27 & 5.96 \\
\hline 29 & 5.76 & 6.28 & 6.40 & 6.33 & 6.36 & 6.62 & 6.58 & 7.34 & 7.05 & 6.31 & 6.27 & 5.96 \\
\hline 30 & 5.75 & 6.30 & 6.40 & 6.33 & - & 6.61 & 6.56 & 7.35 & 6.99 & 6.28 & 6.27 & 5.95 \\
\hline 31 & 5.76 & -- & 6.40 & 6.33 & - & 6.60 & $\cdots$ & 7.46 & - & 6.28 & 6.27 & - \\
\hline MEAN & 5.84 & 6.01 & 6.34 & 6.34 & 6.35 & 6.72 & 6.53 & $\ldots$ & 7.39 & 6.55 & 6.23 & 6.13 \\
\hline MAX & 5.91 & 6.30 & 6.40 & 6.40 & 6.37 & 7.04 & 6.60 & -- & 7.60 & 6.91 & 6.33 & 6.27 \\
\hline MIN & 5.75 & 5.75 & 6.27 & 6.31 & 6.31 & 6.42 & 6.43 & -- & 6.99 & 6.28 & 6.13 & 5.95 \\
\hline
\end{tabular}




\section{GREEN LAKE AT DEEP HOLE NEAR GREEN LAKE, WI}

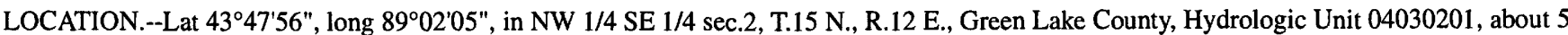
miles southwest of the City of Green Lake.

PERIOD OF RECORD.--May to September 2004. Lake sampled by Wisconsin Department of Natural Resources prior to 2004.

REMARKS.--Water-quality analyses done by Wisconsin State Laboratory of Hygiene.

WATER-QUALITY DATA, MAY 18 TO JULY 23, 2004

(Milligrams per liter unless otherwise indicated)

Date

Lake stage (ft)

Secchi depth (m)

Depth of sample $(\mathrm{m})$

Chlorophyll a, phytoplankton $(\mu \mathrm{g} / \mathrm{L})$

Water temperature $\left({ }^{\circ} \mathrm{C}\right)$

Specific conductance $(\mu \mathrm{S} / \mathrm{cm})$

$\mathrm{pH}$

Dissolved oxygen (mg/L)

Phosphorus, total (as P)

Phosphorus, ortho, dissolved (as $\mathrm{P}$ )

Nitrogen, $\mathrm{NO}_{2}+\mathrm{NO}_{3}$, diss. (as $\mathrm{N}$ )

Nitrogen, ammonia, dissolved (as $\mathrm{N}$ )

Nitrogen, amm. + org., total (as $\mathrm{N}$ )

Color (Pt-Co. scale)

Turbidity (NTU)

Hardness, as $\mathrm{CaCO}_{3}$

Calcium, dissolved $(\mathrm{Ca})$

Magnesium, dissolved (Mg)

Sodium, dissolved (Na)

Potassium, dissolved (K)

Alkalinity, as $\mathrm{CaCO}_{3}$

Sulfate, dissolved $\left(\mathrm{SO}_{4}\right)$

Chloride, dissolved (Cl)

Silica, dissolved $\left(\mathrm{SiO}_{2}\right)$

Solids, dissolved, at $180^{\circ} \mathrm{C}$

Iron, dissolved $(\mathrm{Fe})(\mu \mathrm{g} / \mathrm{L})$

Lead, (Pb) ( $\mu \mathrm{g} / \mathrm{L}$ )

Manganese, dissolved, $(\mathrm{Mn})(\mu \mathrm{g} / \mathrm{L})$

\begin{tabular}{|c|c|c|c|c|c|}
\hline & & & & & \\
\hline & & & & & \\
\hline & & & & & \\
\hline 0.5 & 67.5 & 0.5 & 67 & 0.5 & 67 \\
\hline 30.4 & - & 1.56 & - & 3.07 & - \\
\hline 11.5 & 5.3 & 16.6 & 5.5 & 23.5 & 5.6 \\
\hline 485 & 505 & 485 & 509 & 489 & 508 \\
\hline 8.4 & 7.8 & 8.2 & 7.4 & 8.5 & 7.3 \\
\hline- & - & 8.1 & 6.7 & 10.1 & 2.5 \\
\hline 0.037 & 0.049 & 0.026 & 0.081 & 0.017 & 0.121 \\
\hline 0.002 & - & -- & -- & - & -- \\
\hline$<0.019$ & - & -- & -- & -- & - \\
\hline$<0.015$ & -- & -- & -- & - & -- \\
\hline 0.68 & - & -- & -- & - & - \\
\hline 10 & - & - & -- & -- & -- \\
\hline 2.8 & -- & - & -. & - & - \\
\hline 210 & -. & -- & - & - & - \\
\hline 31.3 & -- & - & - & - & - \\
\hline 32.8 & -- & - & -- & - & - \\
\hline 18.5 & - & - & -- & - & - \\
\hline 3 & -- & - & -- & - & - \\
\hline 180 & -- & -- & -- & - & - \\
\hline 30.5 & - & - & - & - & -- \\
\hline 34.1 & - & - & - & - & - \\
\hline 0.022 & -- & -- & - & - & - \\
\hline 272 & - & - & - & -- & - \\
\hline$<100$ & -- & - & -- & - & - \\
\hline$<1$ & - & - & - & - & - \\
\hline$<1$ & - & - & -- & - & - \\
\hline
\end{tabular}

$5-18-04$
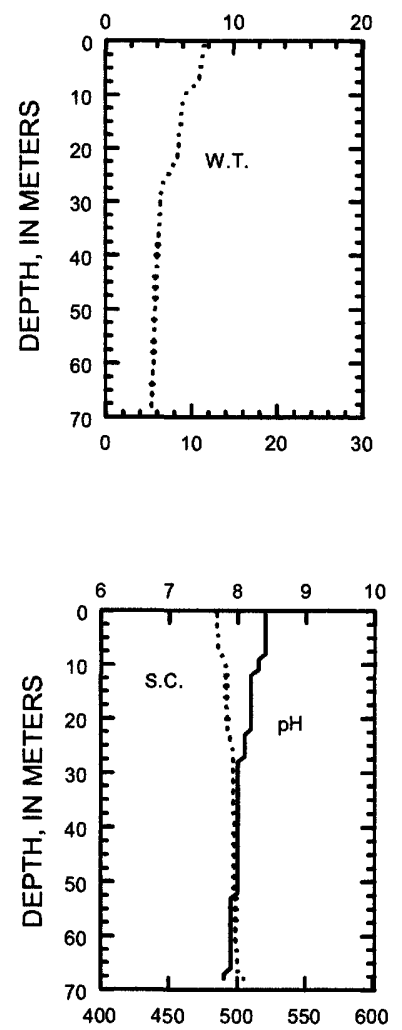

6-25-04

DISSOLVED OXYGEN (D.O. if $_{0}$ IN MILLIGRAMS PER LITER

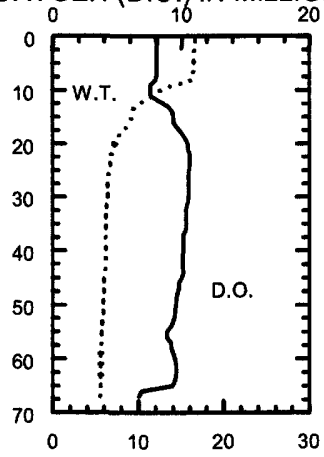

WATER TEMPERATURE (W.T.) IN DEGREES CELSIUS

PH IN STANDARD UNITS

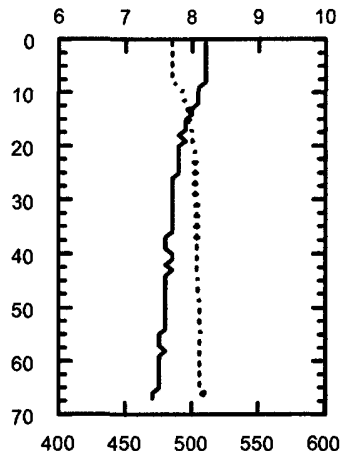

7-23-04
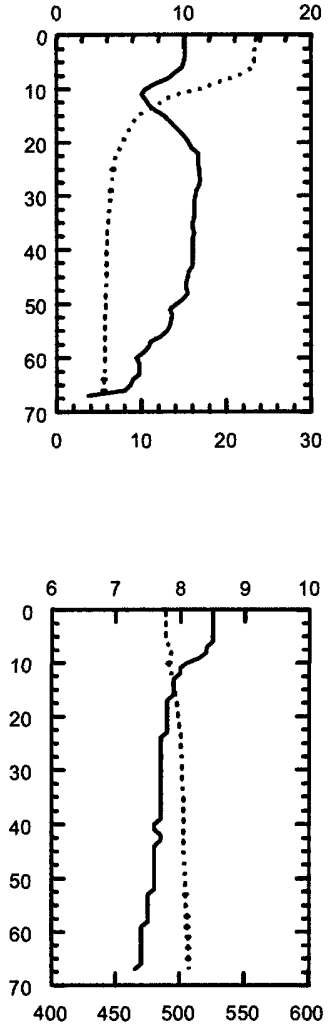
WATER-QUALITY DATA, AUGUST 17 TO SEPTEMBER 16, 2004 (Milligrams per leiter unless otherwise indicated)

Date

Lake stage $(\mathrm{ft})$

Secchi depth $(\mathrm{m})$

Depth of sample $(\mathrm{m})$

Chlorophyll a, phytoplankton ( $\mu \mathrm{g} / \mathrm{L}$ )

Water temperature $\left({ }^{\circ} \mathrm{C}\right)$

Specific conductance $(\mu \mathrm{S} / \mathrm{cm})$

$\mathrm{pH}$

Dissolved oxygen (mg/L)

Phosphorus, total (as P)

Phosphorus, ortho, dissolved (as $\mathrm{P}$ )

Nitrogen, $\mathrm{NO}_{2}+\mathrm{NO}_{3}$, diss. (as $\mathrm{N}$ )

Nitrogen, ammonia, dissolved (as $\mathrm{N}$ )

Nitrogen, amm. + org., total (as N)

Nitrogen, total (as $\mathrm{N}$ )

$\begin{array}{cc}\frac{\text { Sep-16 }}{6.16} & \\ 3.5 & \\ 24 & 67 \\ -- & - \\ 6.9 & 5.6 \\ 496 & 510 \\ 7.7 & 7.3 \\ 10.2 & 0.1 \\ 0.015 & 0.211 \\ -- & - \\ -- & - \\ -- & - \\ - & - \\ -- & -\end{array}$

8-17-04

9-16-04

DISSOLVED OXYGEN (D.O.) IN MILLIGRAMS PER LITER
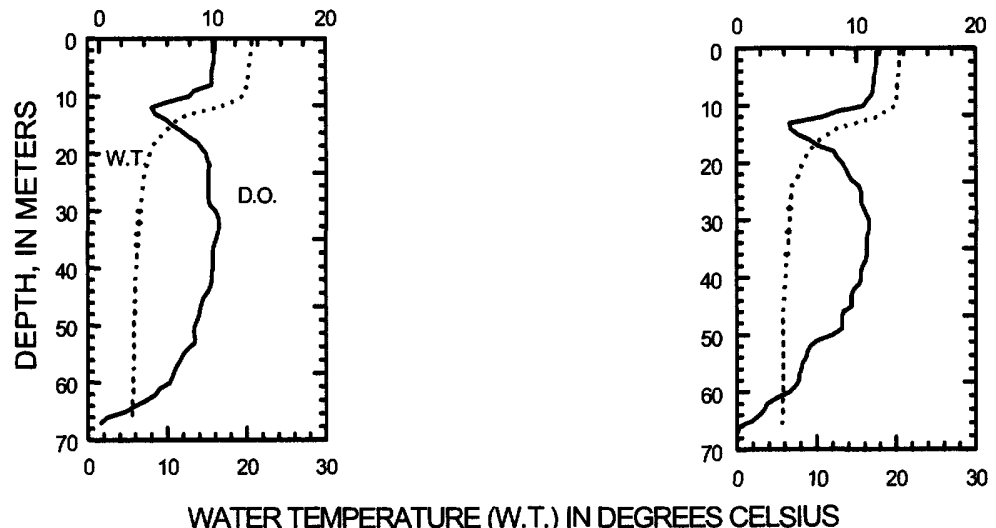

PHIN STANDARD UNITS
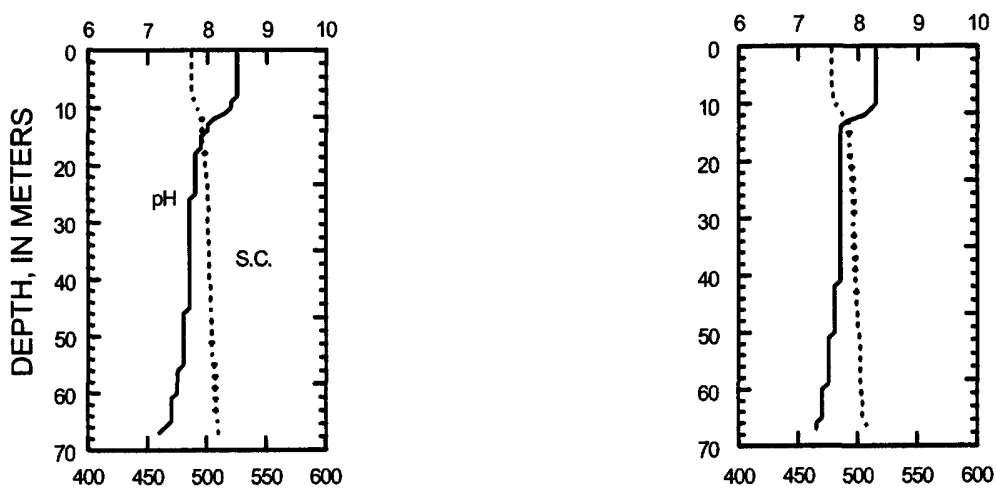

SPECIFIC CONDUCTANCE (S.C.) IN MICROSIEMENS PER CENTIMETER AT 25 DEGREES CELSIUS 

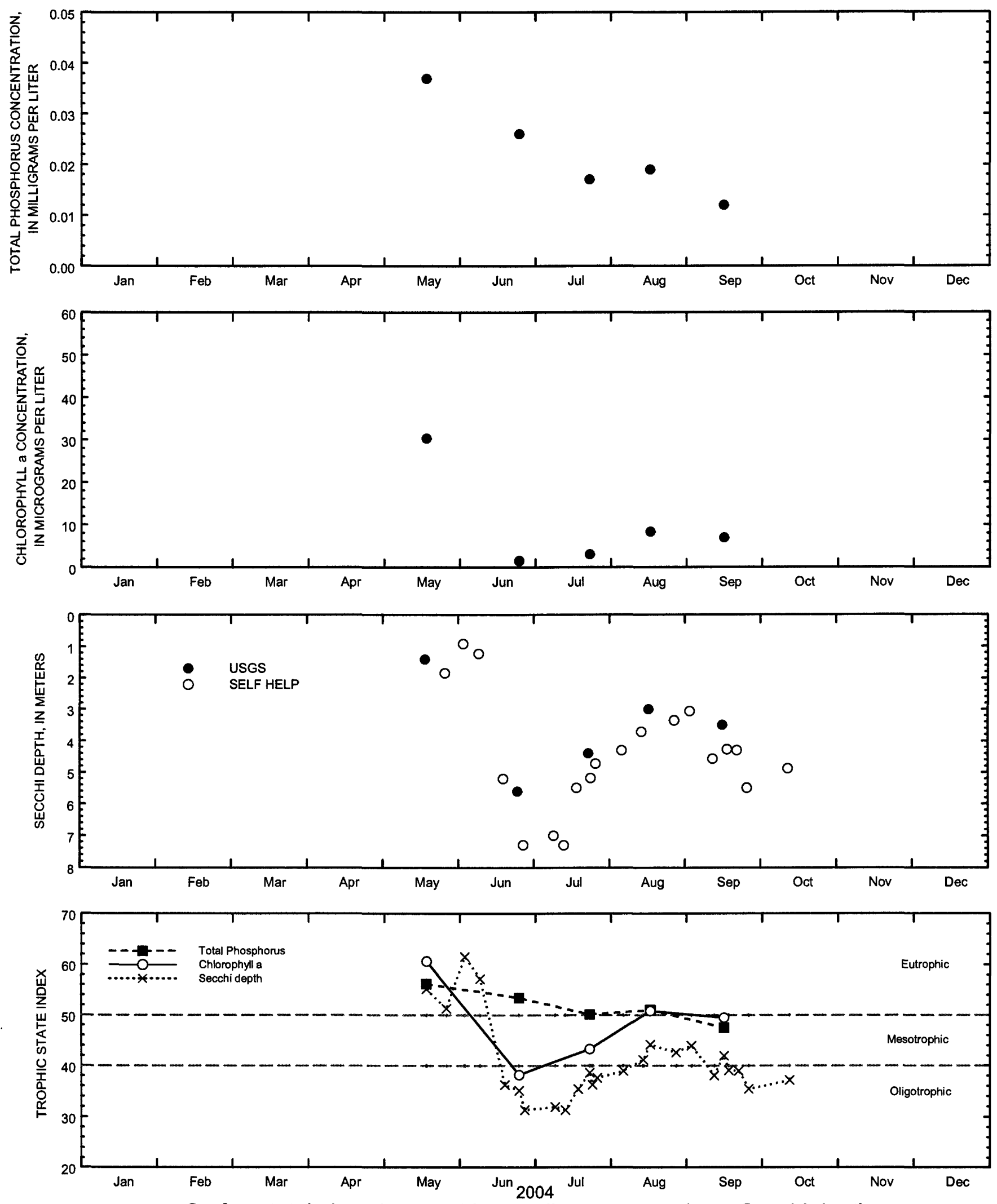

Surface total phosphorus, chlorophyll a concentrations, Secchi depths, and TSI data for Green Lake, Deep Hole, near Green Lake, Wisconsin. 


\section{GREEN LAKE AT EAST END NEAR GREEN LAKE, WI}

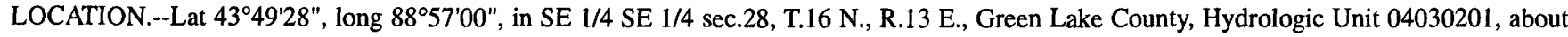
one mile southeast of the City of Green Lake.

PERIOD OF RECORD.--May to September 2004. Lake sampled by Wisconsin Department of Natural Resources prior to 2004.

REMARKS.--Water-quality analyses done by Wisconsin State Laboratory of Hygiene.

WATER-QUALITY DATA, MAY 18 TO SEPTEMBER 16, 2004

(Milligrams per liter unless otherwise indicated)

Date

Lake stage (ft)

Secchi depth (m)

Depth of sample $(\mathrm{m})$

Chlorophyll a, phytoplankton $(\mu \mathrm{g} / \mathrm{L})$

Water temperature $\left({ }^{\circ} \mathrm{C}\right)$

Specific conductance $(\mu \mathrm{S} / \mathrm{cm})$

$\mathrm{pH}$

Dissolved oxygen (mg/L)

Phosphorus, total (as P)

\begin{tabular}{cc}
$\frac{3}{2}$ May-18 \\
\multicolumn{2}{c}{1.83} \\
0.5 & 34 \\
54.5 & - \\
12.1 & 5.8 \\
487 & 500 \\
8.4 & 7.8 \\
- & - \\
0.047 & 0.055
\end{tabular}

\begin{tabular}{cc}
\multicolumn{2}{c}{$\frac{\text { Jun-25 }}{7.27}$} \\
\multicolumn{2}{c}{4.7} \\
0.5 & 32 \\
1.4 & -- \\
19.0 & 6.4 \\
485 & 506 \\
8.2 & 7.5 \\
8.7 & 9.2 \\
0.044 & 0.067
\end{tabular}

\begin{tabular}{cc}
\multicolumn{2}{c}{$\frac{\text { Jul-23 }}{6.43}$} \\
\multicolumn{2}{c}{2.7} \\
0.5 & 34 \\
- & - \\
24.3 & 6.4 \\
500 & 504 \\
8.5 & 7.5 \\
10.7 & 8.6 \\
0.020 & 0.068
\end{tabular}

0.5
8.0
21.1
488
8.5
10.5
0.023

\begin{tabular}{c} 
Aug- 17 \\
\hline 6.19 \\
2.7 \\
23 \\
- \\
7.0 \\
502 \\
7.7 \\
8.9 \\
0.050
\end{tabular}

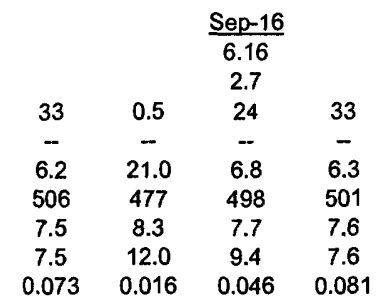

5-18-04

6-25-04

7-23-04

8-17-04

$9-16-04$

DISSOLVED OXYGEN (D.O.) IN MIUIGRAMS PER LITER
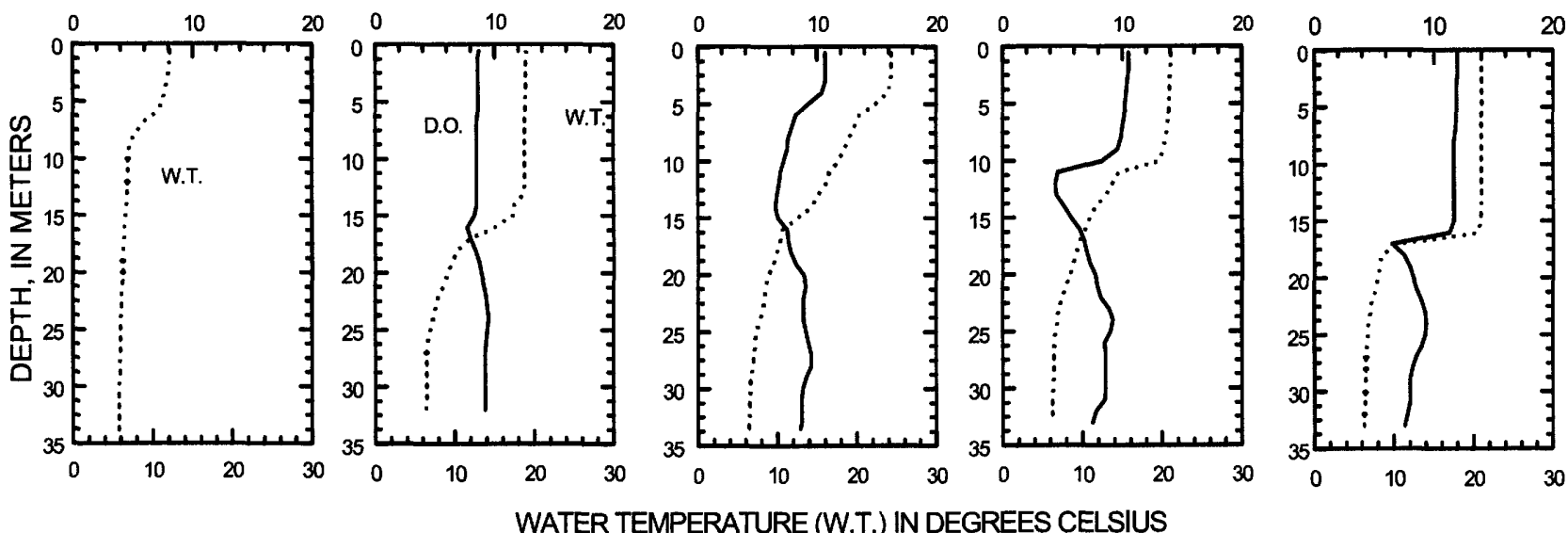

WATER TEMPERATURE (W.T.) IN DEGREES CELSIUS

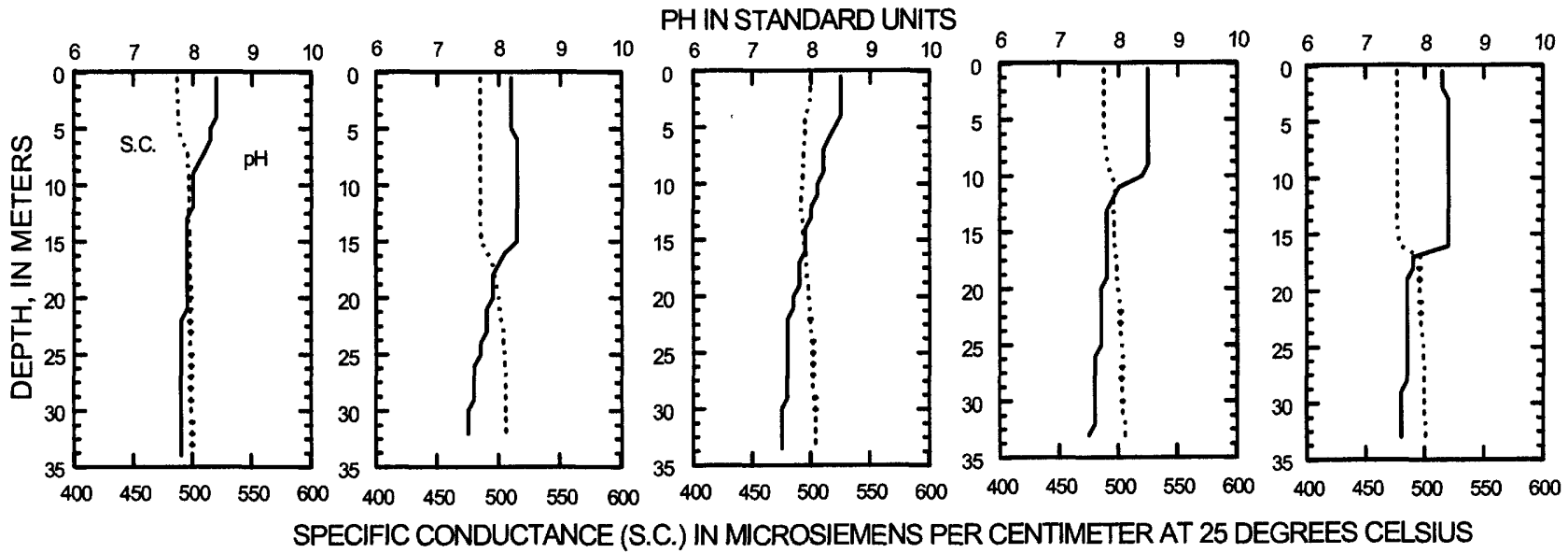



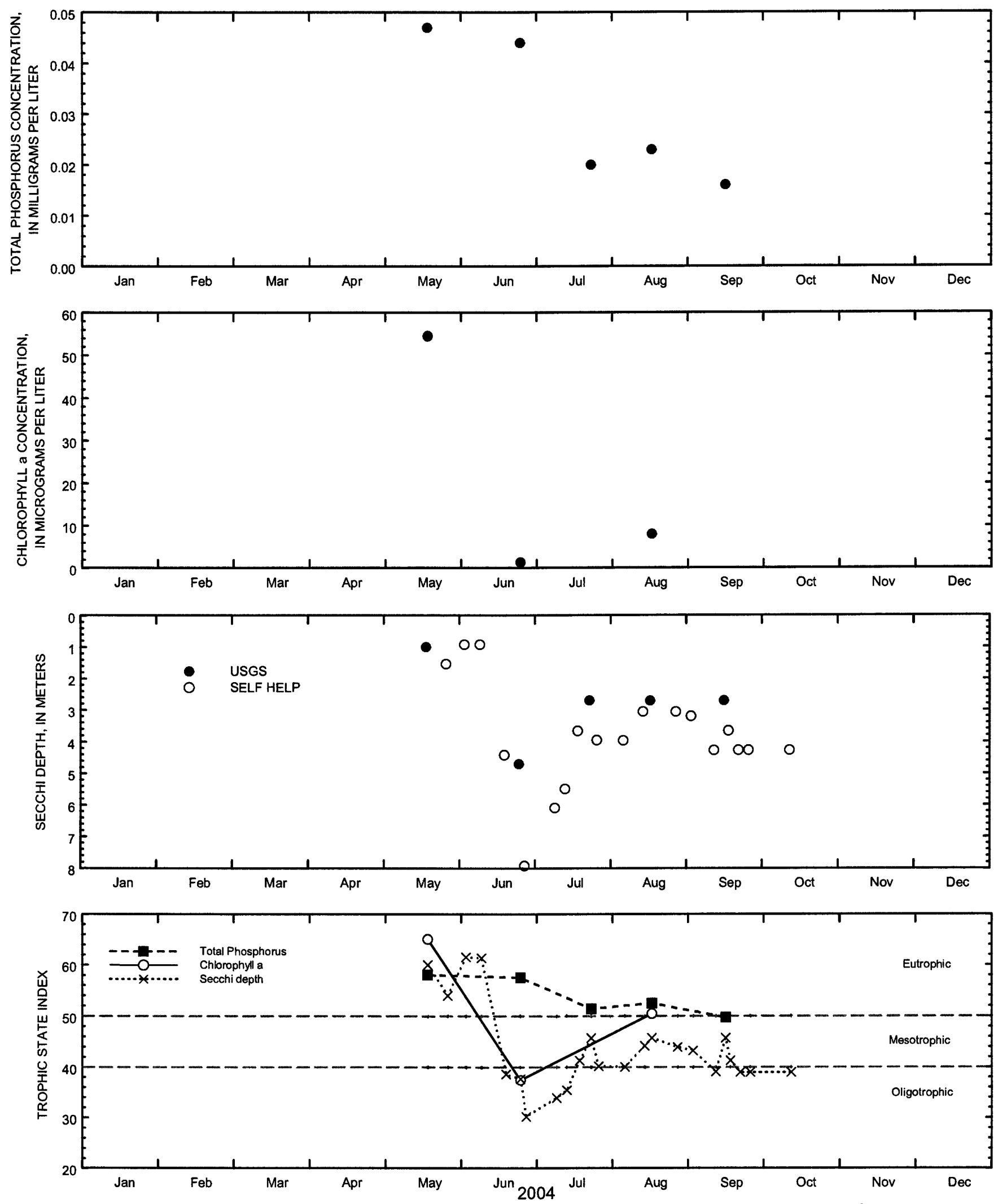

Surface total phosphorus, chlorophyll a concentrations, Secchi depths, and TSI data for Green Lake, East End, near Green Lake, Wisconsin. 


\section{LAKE KOSHKONONG NEAR NEWVILLE, WI}

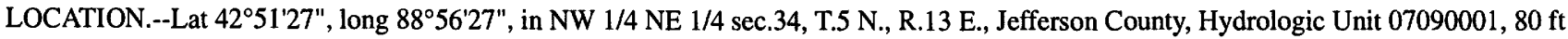
east of Pottawatomi Trail Bridge at Bingham Point Estates, and 4.5 mi northeast of Newville.

DRAINAGE AREA.--2,560 $\mathrm{mi}^{2}$, at lake outlet. Area of Lake Koshkonong, $16.3 \mathrm{mi}^{2}$.

PERIOD OF RECORD.--July 1987 to current year.

GAGE.--Water-stage recorder. Datum of gage is $770.00 \mathrm{ft}$ above sea level.

REMARKS.--Lake level regulated by dam at Indianford. Gage-height telemeter at station.

EXTREMES FOR PERIOD OF RECORD.--Maximum recorded gage height, $12.23 \mathrm{ft}$, Apr. 25, 1993; minimum recorded, $5.10 \mathrm{ft}$, Dec. 28 , 29, 1999.

EXTREMES FOR CURRENT YEAR.--Maximum recorded gage height, $11.49 \mathrm{ft}$, June 5; minimum daily gage height, $5.66 \mathrm{ft}$, Feb. 13.

GAGE HEIGHT, FEET

WATER YEAR OCTOBER 2003 TO SEPTEMBER 2004

DAILY MEAN VALUES

\begin{tabular}{|c|c|c|c|c|c|c|c|c|c|c|c|c|}
\hline DAY & OCT & NOV & DEC & JAN & FEB & MAR & APR & MAY & JUN & JUL & AUG & SEP \\
\hline 1 & 6.12 & 5.90 & 6.42 & 5.85 & 5.80 & 6.01 & 8.16 & 6.70 & 11.12 & 10.60 & 6.84 & 6.31 \\
\hline 2 & 6.09 & 5.94 & 6.37 & 5.80 & 5.81 & 6.09 & 8.16 & 6.63 & 11.30 & 10.46 & 6.75 & 6.29 \\
\hline 3 & 6.09 & 6.02 & 6.34 & 5.81 & 5.85 & 6.20 & 8.17 & 6.55 & 11.42 & 10.34 & 6.66 & 6.27 \\
\hline 4 & 6.10 & 6.22 & 6.30 & 5.81 & 5.90 & 6.34 & 8.08 & 6.47 & 11.48 & 10.30 & 6.76 & 6.27 \\
\hline 5 & 6.08 & 6.34 & 6.26 & 5.75 & 5.91 & 6.65 & 8.01 & 6.39 & 11.49 & 10.17 & 6.78 & 6.26 \\
\hline 6 & 6.08 & 6.44 & 6.21 & 5.75 & 5.88 & 6.96 & 7.96 & 6.31 & 11.46 & 10.04 & 6.77 & 6.26 \\
\hline 7 & 6.08 & 6.57 & 6.14 & 5.76 & 5.84 & 7.29 & 7.91 & 6.21 & 11.40 & 9.95 & 6.73 & 6.24 \\
\hline 8 & 6.08 & 6.63 & 6.10 & 5.77 & 5.80 & 7.59 & 7.86 & 6.18 & 11.33 & 9.83 & 6.70 & 6.21 \\
\hline 9 & 6.08 & 6.70 & 6.07 & 5.77 & 5.75 & 7.82 & 7.78 & 6.25 & 11.23 & 9.71 & 6.69 & 6.24 \\
\hline 10 & 6.08 & 6.79 & 6.20 & 5.77 & 5.71 & 7.99 & 7.71 & 6.31 & 11.16 & 9.61 & 6.66 & 6.26 \\
\hline 11 & 6.08 & 6.90 & 6.23 & 5.78 & 5.68 & 8.10 & 7.61 & 6.45 & 11.17 & 9.49 & 6.59 & 6.30 \\
\hline 12 & 6.11 & 7.04 & 6.24 & 5.78 & 5.67 & 8.17 & 7.51 & 6.54 & 11.17 & 9.38 & 6.47 & 6.33 \\
\hline 13 & 6.09 & 7.06 & 6.27 & 5.78 & 5.66 & 8.21 & 7.42 & 6.69 & 11.20 & 9.28 & 6.36 & 6.33 \\
\hline 14 & 6.16 & 6.97 & 6.31 & 5.78 & 5.68 & 8.27 & 7.31 & 6.85 & 11.28 & 9.17 & 6.27 & 6.30 \\
\hline 15 & 6.14 & 6.92 & 6.35 & 5.76 & 5.70 & 8.25 & 7.19 & 6.96 & 11.32 & 9.05 & 6.27 & 6.27 \\
\hline 16 & 6.13 & 6.88 & 6.33 & 5.74 & 5.71 & 8.24 & 7.10 & 7.05 & 11.34 & 8.94 & 6.26 & 6.30 \\
\hline 17 & 6.12 & 6.82 & 6.29 & 5.74 & 5.72 & 8.22 & 7.03 & 7.15 & 11.44 & 8.82 & 6.29 & 6.26 \\
\hline 18 & 6.12 & 6.83 & 6.23 & 5.72 & 5.73 & 8.20 & 6.92 & 7.28 & 11.45 & 8.68 & 6.29 & 6.23 \\
\hline 19 & 6.12 & 6.76 & 6.17 & 5.70 & 5.74 & 8.16 & 6.88 & 7.35 & 11.43 & 8.55 & 6.27 & 6.20 \\
\hline 20 & 6.13 & 6.68 & 6.08 & 5.70 & 5.77 & 8.16 & 6.75 & 7.42 & 11.39 & 8.42 & 6.23 & 6.19 \\
\hline 21 & 6.15 & 6.59 & 5.99 & 5.69 & 5.80 & 8.07 & 6.84 & 7.54 & 11.37 & 8.31 & 6.22 & 6.21 \\
\hline 22 & 6.13 & 6.49 & 5.94 & 5.69 & 5.82 & 7.99 & 6.86 & 8.03 & 11.36 & 8.22 & 6.20 & 6.23 \\
\hline 23 & 6.13 & 6.48 & 5.94 & 5.69 & 5.86 & 7.94 & 6.88 & 8.57 & 11.29 & 8.09 & 6.21 & 6.24 \\
\hline 24 & 6.13 & 6.51 & 5.92 & 5.69 & 5.90 & 7.91 & 6.88 & 9.11 & 11.26 & 7.94 & 6.25 & 6.26 \\
\hline 25 & 6.18 & 6.43 & 5.91 & 5.68 & 5.91 & 7.87 & 6.91 & 9.58 & 11.19 & 7.81 & 6.29 & 6.27 \\
\hline 26 & 6.19 & 6.44 & 5.90 & 5.67 & 5.89 & 7.94 & 6.92 & 9.96 & 11.12 & 7.67 & 6.30 & 6.27 \\
\hline 27 & 6.17 & 6.43 & 5.91 & 5.68 & 5.90 & 7.94 & 6.85 & 10.25 & 11.03 & 7.51 & 6.35 & 6.27 \\
\hline 28 & 6.12 & 6.46 & 5.95 & 5.71 & 5.91 & 7.97 & 6.78 & 10.45 & 10.96 & 7.36 & 6.37 & 6.29 \\
\hline 29 & 6.05 & 6.40 & 5.97 & 5.74 & 5.94 & 8.06 & 6.77 & 10.60 & 10.84 & 7.21 & 6.37 & 6.25 \\
\hline 30 & 5.99 & 6.42 & 5.95 & 5.76 & --. & 8.14 & 6.74 & 10.75 & 10.72 & 7.07 & 6.36 & 6.25 \\
\hline 31 & 5.96 & -- & 5.91 & 5.78 & -- & 8.15 & -- & 10.93 & -- & 6.95 & 6.33 & -- \\
\hline MEAN & 6.11 & 6.57 & 6.14 & 5.75 & 5.80 & 7.71 & 7.33 & 7.73 & 11.26 & 8.87 & 6.45 & 6.26 \\
\hline MAX & 6.19 & 7.06 & 6.42 & 5.85 & 5.94 & 8.27 & 8.17 & 10.93 & 11.49 & 10.60 & 6.84 & 6.33 \\
\hline MIN & 5.96 & 5.90 & 5.90 & 5.67 & 5.66 & 6.01 & 6.74 & 6.18 & 10.72 & 6.95 & 6.20 & 6.19 \\
\hline
\end{tabular}


LOCATION.--Lat $43^{\circ} 22^{\prime} 55^{\prime \prime}$, long $88^{\circ} 13^{\prime} 47^{\prime \prime}$, in NW 1/4 NE 1/4 sec.33, T.11 N., R.19 E., Washington County, Hydrologic Unit 04040003, 2.6 mi southwest of West Bend.

PERIOD OF RECORD.--February 1997 to August 1999, February 2003 to current year.

REMARKS.--Lake sampled at center of northern basin at deep hole. Lake ice-covered during February sampling. Water-quality analyses done by Wisconsin State Laboratory of Hygiene.

WATER-QUALITY DATA, FEBRUARY 24 TO AUGUST 18, 2004

(Milligrams per liter unless otherwise indicated)

Date

Lake stage (ft)

Secchi depth $(m)$

Depth of sample $(\mathrm{m})$

Chlorophyll a, phytoplankton $(\mu \mathrm{g} / \mathrm{L})$

Water temperature $\left({ }^{\circ} \mathrm{C}\right)$

Specific conductance $(\mu \mathrm{S} / \mathrm{cm})$

$\mathrm{pH}$

Dissolved oxygen (mg/L)

Phosphorus, total (as P)

\begin{tabular}{cccc}
\multicolumn{2}{c}{ Feb-24 } & \multicolumn{2}{c}{ Apr-22 } \\
\multicolumn{2}{c}{--} \\
0.5 & 6.5 & 0.5 & 7.5 \\
- & -- & 28.0 & - \\
0.6 & 4.0 & 12.0 & 9.8 \\
589 & 620 & 538 & 545 \\
7.6 & 7.2 & 8.5 & 8.1 \\
12.3 & 4.3 & 10.9 & 9.0 \\
0.015 & 0.025 & 0.041 & 0.035
\end{tabular}

$\begin{array}{lc}\frac{\text { Jun- } 9}{4.86} \\ \\ 2.2 & \\ 5 & 7.5 \\ 0 & -- \\ 2 & 13.5 \\ 4 & 531 \\ 4 & 7.4 \\ 4 & 0.5 \\ 28 & 0.056\end{array}$

\begin{tabular}{cc}
\multicolumn{3}{c}{$\frac{\text { Jul-14 }}{4.98}$} \\
\multicolumn{2}{c}{2.0} \\
0.5 & 7.5 \\
8.2 & -- \\
24.6 & 13.5 \\
515 & 557 \\
8.2 & 7.2 \\
11.3 & 0.3 \\
0.024 & 0.302
\end{tabular}

6-9-04

7-14-04

8-18-04

DISSOLVED OXYGEN (D.O.) IN MILIGRAMS PER LITER
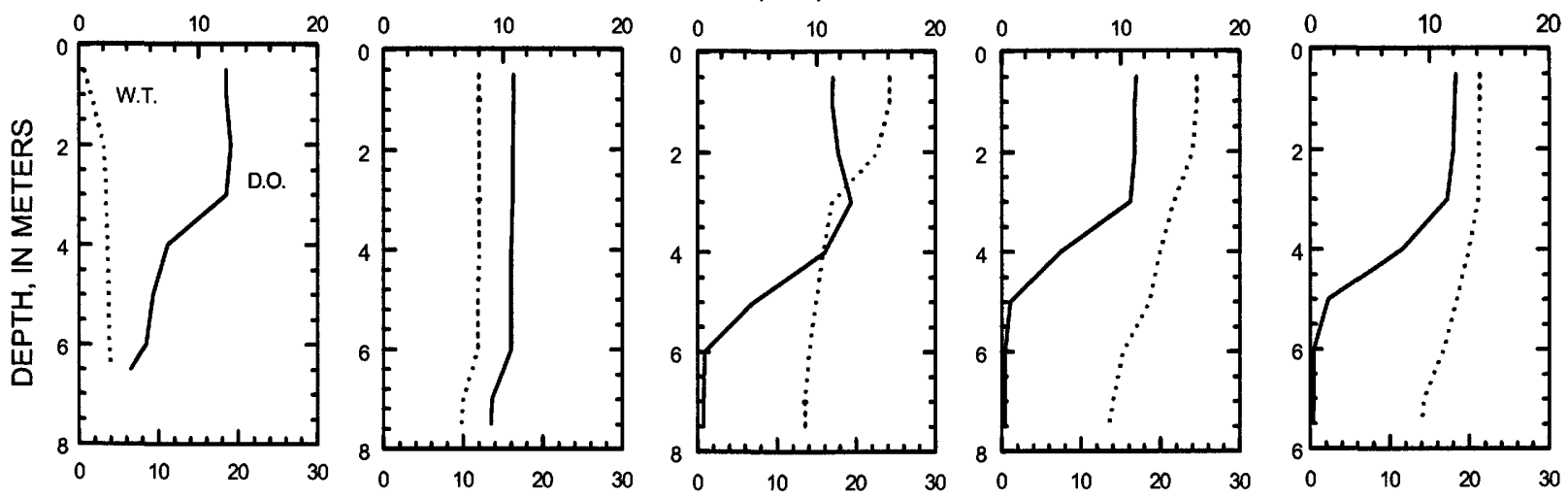

WATER TEMPERATURE (W.T.) IN DEGREES CELSIUS

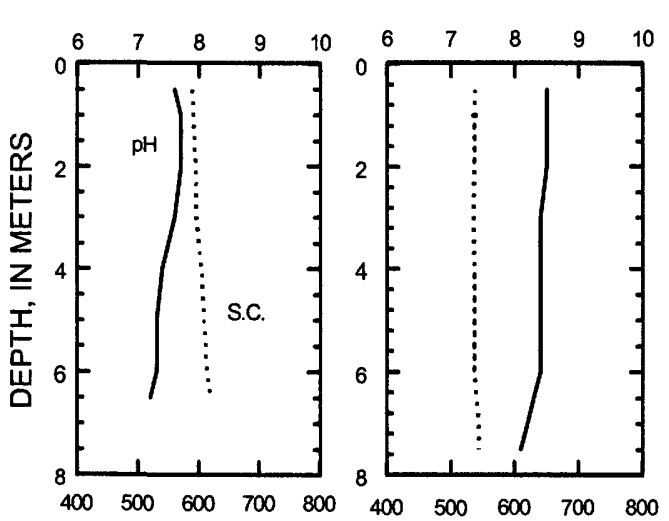

PHIN STANDARD UNITS
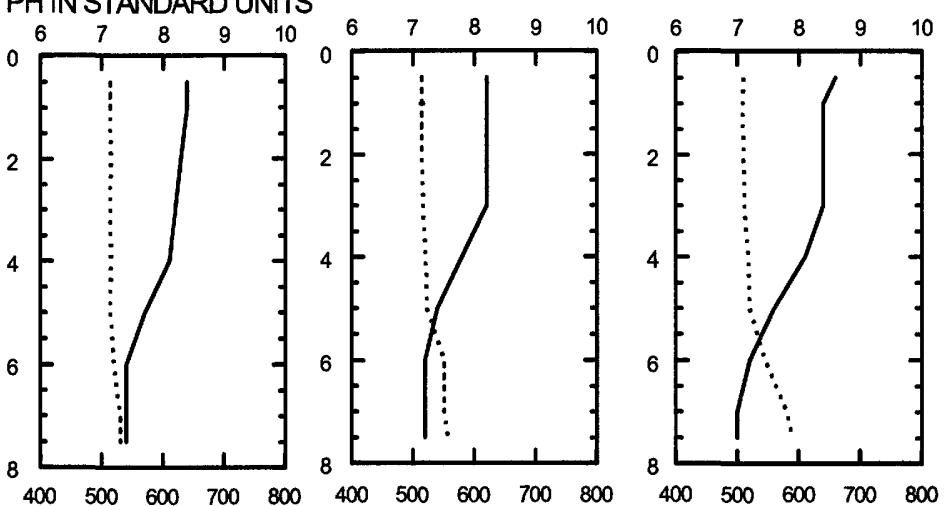

SPECIFIC CONDUCTANCE (S.C.) IN MICROSIEMENS PER CENTIMETER AT 25 DEGREES CELSIUS 

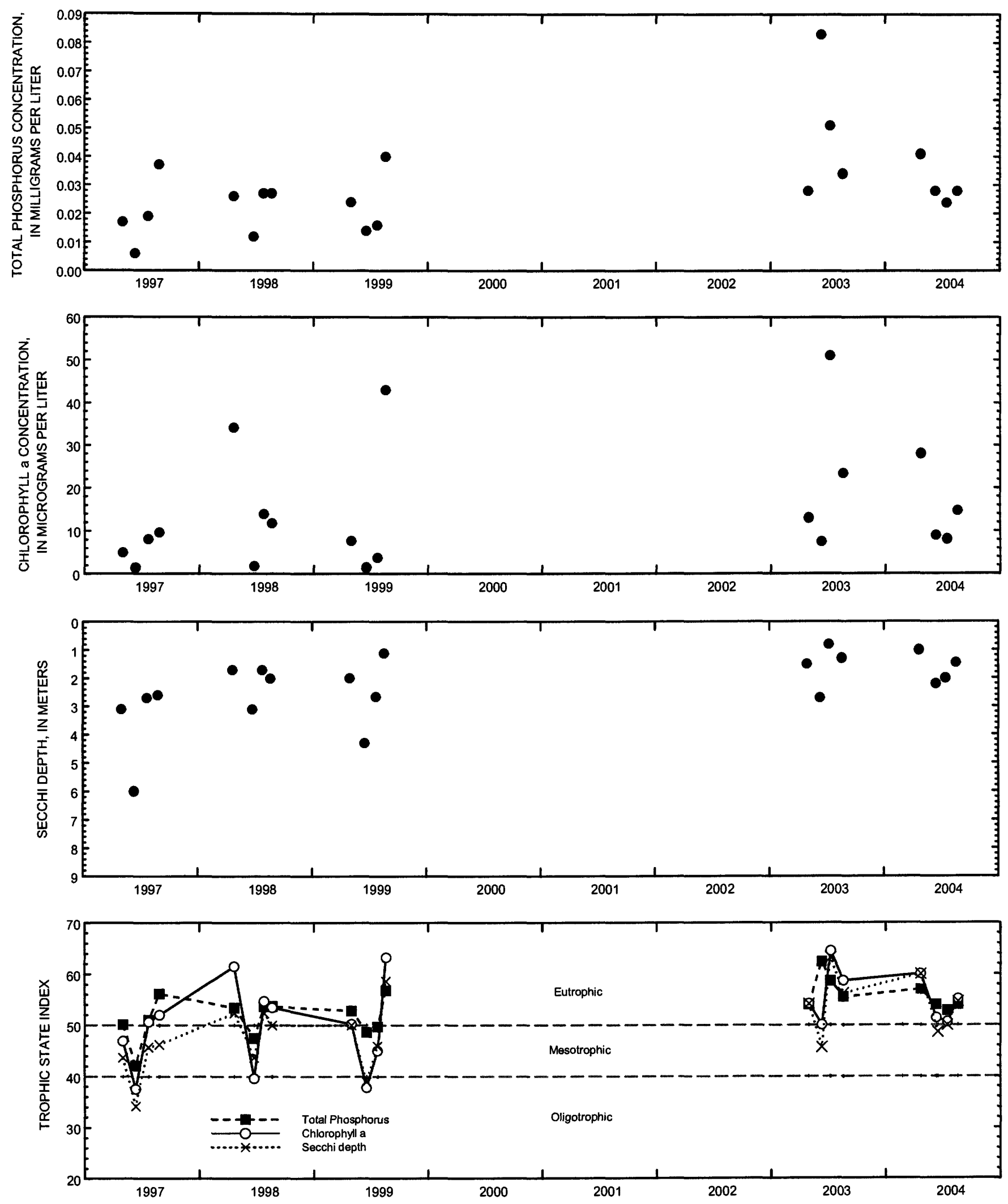

Surface total phosphorus, chlorophyll a concentrations, Secchi depths, and TSI data for Little Cedar Lake, North Site, near West Bend, Wisconsin. 


\section{LITTLE CEDAR LAKE, SOUTH SITE, NEAR WEST BEND, WI}

LOCATION.--Lat $43^{\circ} 22^{\prime} 49^{\prime \prime}$, long $88^{\circ} 13^{\prime} 45^{\prime \prime}$, in NW 1/4 SE 1/4 sec.33, T.11 N., R.19 E., Washington County, Hydrologic Unit 04040003, 2.8 mi southwest of West Bend.

PERIOD OF RECORD.--February 1997 to current year

REMARKS.--Lake sampled in southern basin at deep hole. Lake ice-covered during February sampling. Water-quality analyses done by Wisconsin State Laboratory of Hygiene.

WATER-QUALITY DATA, FEBRUARY 24 TO AUGUST 18, 2004

(Milligrams per liter unless otherwise indicated)

Date

Lake stage (ft)

Secchi depth $(\mathrm{m})$

Depth of sample (m)

Chlorophyll a, phytoplankton $(\mu g / L)$

Water temperature $\left({ }^{\circ} \mathrm{C}\right)$

Specific conductance $(\mu \mathrm{S} / \mathrm{cm})$

$\mathrm{pH}$

Dissolved oxygen (mg/L)

Phosphorus, total (as P)

Phosphorus, ortho, dissolved (as $P$ )

Nitrogen, $\mathrm{NO}_{2}+\mathrm{NO}_{3}$, diss. (as $\mathrm{N}$ )

Nitrogen, ammonia, dissolved (as $\mathrm{N}$ )

Nitrogen, amm. + diss., total (as $\mathrm{N}$ )

Nitrogen, amm. + org., total (as N)

Color (Pt-Co. scale)

Turbidity (NTU)

Hardness, as $\mathrm{CaCO}_{3}$

Calcium, dissolved ( $\mathrm{Ca}$ )

Magnesium, dissolved (Mg)

Sodium, dissolved $(\mathrm{Na})$

Potassium, dissolved $(\mathrm{K})$

Alkalinity, as $\mathrm{CaCO}_{3}$

Sulfate, dissolved $\left(\mathrm{SO}_{4}\right)$

Chloride, dissolved $(\mathrm{Cl})$

Silica, dissolved $\left(\mathrm{SiO}_{2}\right)$

Solids, dissolved, at $180^{\circ} \mathrm{C}$

Iron, dissolved $(\mathrm{Fe})(\mu \mathrm{g} / \mathrm{L})$

Manganese, dissolved, $(M \pi)(\mu g / L)$

\begin{tabular}{|c|c|c|}
\hline & & Apr-22 \\
\hline & & -- \\
\hline & & 1.0 \\
\hline 0.5 & 16.5 & 0.5 \\
\hline- & - & 33.4 \\
\hline 0.8 & 4.2 & 11.5 \\
\hline 560 & 623 & 532 \\
\hline 8.0 & 7.1 & 8.5 \\
\hline 15.0 & 0.6 & 11.6 \\
\hline 0.031 & 0.113 & 0.038 \\
\hline- & - & 0.003 \\
\hline- & -- & $<0.019$ \\
\hline- & - & $<0.015$ \\
\hline- & - & -- \\
\hline- & - & 0.83 \\
\hline- & - & 15 \\
\hline- & -- & 4.7 \\
\hline- & - & 220 \\
\hline- & - & 34.6 \\
\hline- & - & 32.2 \\
\hline- & - & 21 \\
\hline- & - & 2 \\
\hline - & - & 185 \\
\hline- & - & 18 \\
\hline - & - & 45.7 \\
\hline- & - & 2.97 \\
\hline- & - & 300 \\
\hline- & - & $<100$ \\
\hline- & - & $<1$ \\
\hline
\end{tabular}

\begin{tabular}{|c|c|c|c|c|c|c|}
\hline & & & & & Aug-18 & \\
\hline & & & & & - & \\
\hline & & & & & 1.8 & \\
\hline 0.5 & 16.5 & 0.5 & 16.5 & 0.5 & 8 & 16.5 \\
\hline 12.7 & - & 5.8 & -- & 8.4 & - & - \\
\hline 23.6 & 8.6 & 24.4 & 8.8 & 21.3 & 12.8 & 8.8 \\
\hline 502 & 536 & 505 & 554 & 508 & 534 & 566 \\
\hline 8.3 & 7.5 & 8.2 & 7.3 & 8.4 & 7.5 & 7.2 \\
\hline 10.6 & 0.3 & 11.8 & 0.3 & 11.5 & 0.4 & 0.2 \\
\hline 0.040 & 0.116 & 0.013 & 0.256 & 0.015 & 0.080 & 0.284 \\
\hline- & - & $<0.002$ & -- & - & - & - \\
\hline -- & -- & $<0.019$ & -- & -- & - & -- \\
\hline -- & -- & $<0.015$ & -- & - & - & -- \\
\hline- & - & 0.64 & -- & - & -- & - \\
\hline -- & -- & -- & - & -- & -- & - \\
\hline -- & -- & - & -- & - & -- & - \\
\hline- & -- & - & -- & - & -- & - \\
\hline- & -- & -- & .. & - & -- & - \\
\hline -- & -- & - & -- & - & -- & - \\
\hline- & - & - & - & - & - & - \\
\hline- & -- & - & -- & - & -- & - \\
\hline -- & - & - & - & -- & - & - \\
\hline- & -- & - & -- & - & - & -- \\
\hline- & -- & - & -- & -- & - & - \\
\hline- & -- & - & -- & -- & - & -- \\
\hline- & -- & - & - & -- & - & -- \\
\hline- & -- & - & - & -- & -- & - \\
\hline- & -- & - & - & - & - & -. \\
\hline -- & - & - & -. & -- & -- & -- \\
\hline
\end{tabular}

4-22-04

6-9-04

7-14-04

8-18-04

DISSOLVED OXYGEN (D.O.) IN MILLIGRAMS PER LITER
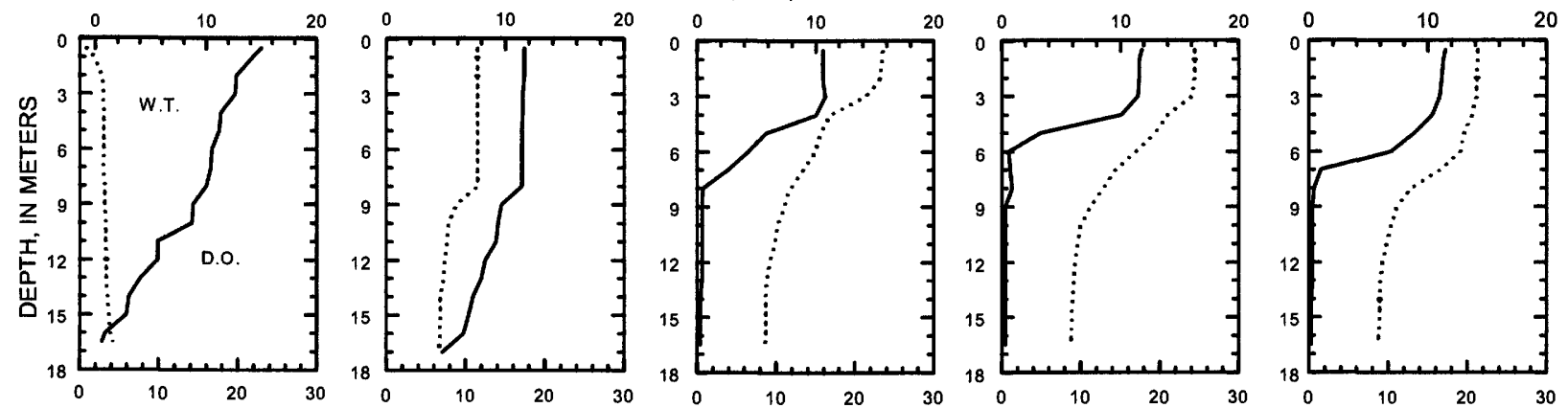

WATER TEMPERATURE (W.T.) IN DEGREES CELSIUS

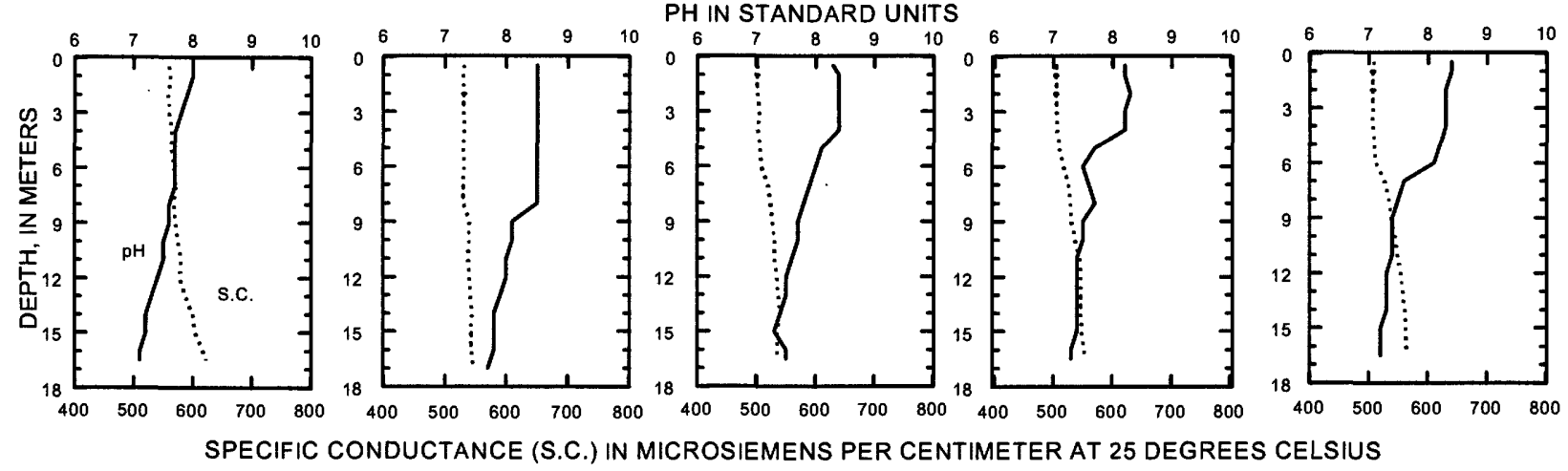



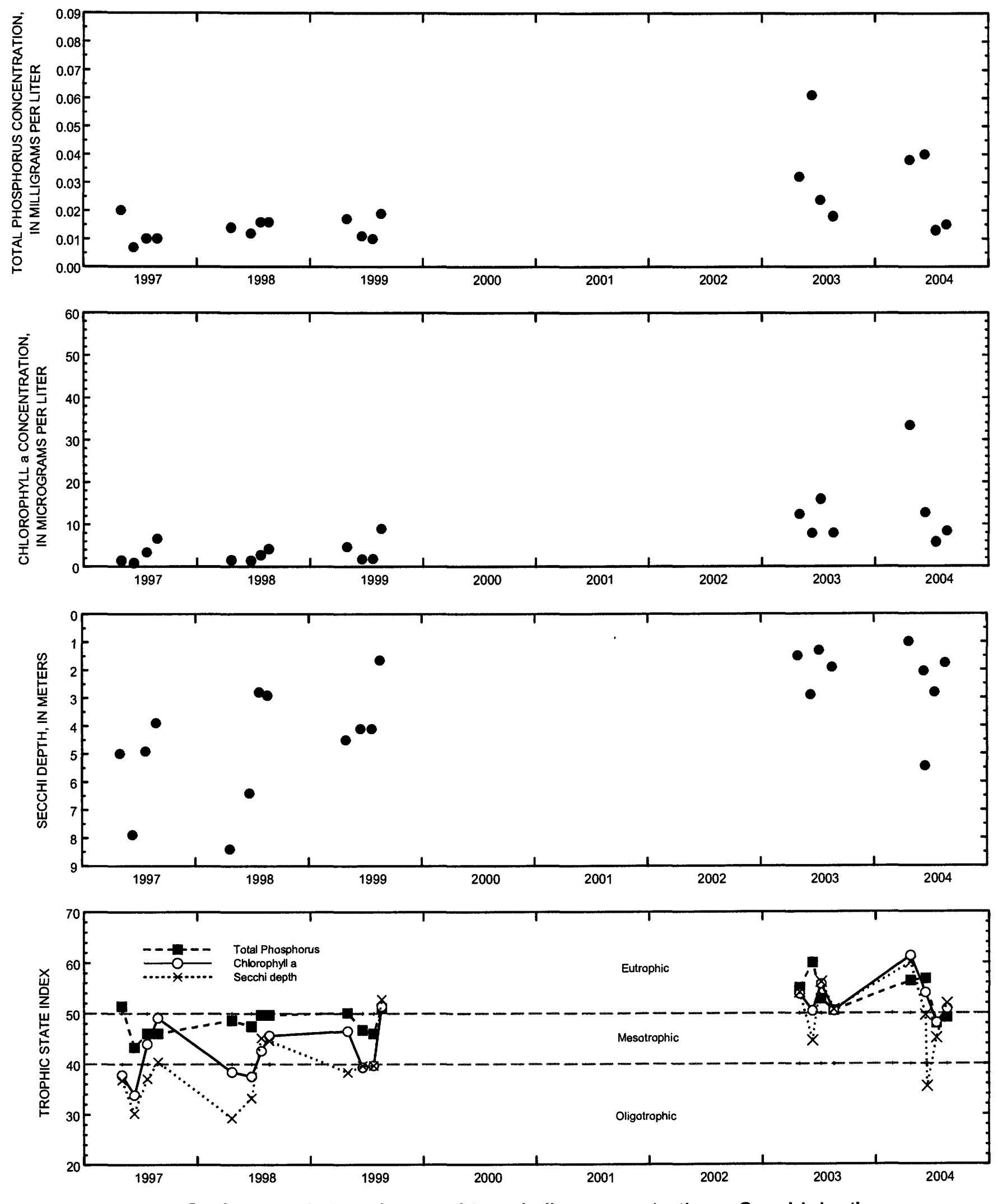

Surface total phosphorus, chlorophyll a concentrations, Secchi depths, and TSI data for Little Cedar Lake, South Site, near West Bend, Wisconsin. 


\section{LAKE MENDOTA AT MADISON, WI}

LOCATION.--Lat 4305' $42^{\prime \prime}$, long $89^{\circ} 22^{\prime} 12^{\prime \prime}$, in SE 1/4 sec.12, T.7 N., R.9 E., Dane County, Hydrologic Unit 07090001, in county boat house at dam at outlet, in Madison.

DRAINAGE AREA.--233 $\mathrm{mi}^{2}$. Area of Lake Mendota, $15.2 \mathrm{mi}^{2}$.

PERIOD OF RECORD.--January 1916 to current year (incomplete).

REVISED RECORDS.--WDR WI-73-1: Drainage area.

GAGE.--Water-stage recorder. Datum of gage is $840.00 \mathrm{ft}$ above sea level, or $5.60 \mathrm{ft}$ below City of Madison datum. Prior to Oct. 1, 1979, at datum $7.82 \mathrm{ft}$ higher; prior to Nov. 15, 1971, nonrecording gage at same site.

REMARKS.--Lake level regulated by concrete dam with two 12-foot gates and 20-foot lock at outlet. Gage-height telemeter at station.

EXTREMES FOR PERIOD OF RECORD.--Maximum gage height observed, $12.75 \mathrm{ft}$, June 5, 2000; minimum observed, $8.02 \mathrm{ft}$, Feb. 24 to Mar. 10, 1920, current datum.

EXTREMES FOR CURRENT YEAR.--Maximum recorded gage height, $11.61 \mathrm{ft}$, May 26; minimum recorded, 8.87 ft, Feb. $18,19$.

\section{WATER YEAR OCTOBER 2003 TO SEPTEMBER 2004 DAILY MEAN VALUES}

\begin{tabular}{|c|c|c|c|c|c|c|c|c|c|c|c|c|}
\hline DAY & OCT & NOV & DEC & JAN & FEB & MAR & APR & MAY & JUN & JUL & AUG & SEF \\
\hline 1 & 9.72 & 9.26 & 9.56 & 9.39 & 9.01 & 9.16 & 9.99 & 9.74 & 11.57 & 10.81 & 10.26 & 70. \\
\hline 2 & 9.68 & 9.33 & 9.49 & 9.39 & 9.01 & 9.22 & 9.96 & 9.73 & 11.53 & 10.79 & 10.30 & 10.0 \\
\hline 3 & 9.67 & 9.43 & 9.44 & 9.39 & 9.01 & 9.26 & 9.96 & 9.72 & 11.48 & 10.78 & 10.34 & 10.0 \\
\hline 4 & 9.66 & 9.84 & 9.43 & 9.36 & 9.00 & 9.30 & 9.89 & 9.71 & 11.41 & 11.03 & 10.49 & 10.0 \\
\hline 5 & 9.64 & 9.98 & 9.44 & 9.35 & 9.00 & 9.48 & 9.85 & 9.70 & 11.34 & 11.02 & 10.49 & 10. \\
\hline 6 & 9.63 & 10.04 & 9.42 & 9.30 & 9.02 & 9.60 & 9.84 & 9.70 & 11.28 & 10.96 & 10.49 & \\
\hline 7 & 9.63 & 10.07 & 9.39 & 9.29 & 9.01 & 9.66 & 9.82 & 9.69 & 11.21 & 10.95 & 10.47 & \\
\hline 8 & 9.64 & 10.06 & 9.39 & 9.29 & 8.99 & 9.69 & 9.80 & 9.69 & 11.16 & 10.92 & 10.43 & \\
\hline 9 & 9.65 & 10.03 & 9.39 & 9.27 & 8.98 & 9.70 & 9.75 & 9.76 & 11.11 & 10.85 & 10.43 & \\
\hline 10 & 9.64 & 9.99 & 9.56 & 9.26 & 8.97 & 9.70 & 9.72 & 9.81 & 11.08 & 10.81 & 10.38 & \\
\hline 11 & 9.65 & 9.98 & 9.59 & 9.26 & 8.96 & 9.70 & 9.67 & 9.83 & 11.17 & 10.75 & 10.31 & \\
\hline 12 & 9.66 & 10.00 & 9.57 & 9.24 & 8.95 & 9.70 & 9.64 & 9.85 & 11.22 & 10.72 & 10.24 & \\
\hline 13 & 9.64 & 9.93 & 9.56 & 9.23 & 8.94 & 9.70 & 9.63 & 9.89 & 11.25 & 10.68 & 10.21 & \\
\hline 14 & 9.67 & 9.87 & 9.56 & 9.22 & 8.93 & 9.71 & 9.62 & 9.94 & 11.25 & 10.61 & 10.18 & \\
\hline 15 & 9.63 & 9.84 & 9.55 & 9.21 & 8.91 & 9.72 & 9.61 & 9.94 & 11.22 & 10.54 & 10.15 & \\
\hline 16 & 9.59 & 9.81 & 9.59 & 9.20 & 8.90 & 9.71 & 9.61 & 9.90 & 11.19 & 10.50 & 10.10 & \\
\hline 17 & 9.55 & 9.77 & 9.56 & 9.20 & 8.89 & 9.72 & 9.66 & 9.94 & 11.20 & 10.48 & 10.09 & \\
\hline 18 & 9.52 & 9.81 & 9.55 & 9.18 & 8.87 & 9.73 & 9.68 & 10.02 & 11.17 & 10.42 & 10.09 & \\
\hline 19 & 9.50 & 9.82 & 9.54 & 9.17 & 8.87 & 9.73 & 9.71 & 10.02 & 11.08 & 10.38 & 10.08 & 9. \\
\hline 20 & 9.48 & 9.76 & 9.51 & 9.16 & 8.89 & 9.75 & 9.70 & 10.05 & 11.01 & 10.34 & 10.05 & \\
\hline 21 & 9.46 & 9.71 & 9.50 & 9.14 & 8.90 & 9.73 & 9.77 & 10.14 & 10.96 & 10.32 & 10.02 & \\
\hline 22 & 9.41 & 9.66 & 9.50 & 9.11 & 8.90 & 9.72 & 9.77 & 10.67 & 10.92 & 10.29 & 10.00 & \\
\hline 23 & 9.39 & 9.81 & 9.49 & 9.11 & 8.91 & 9.72 & 9.76 & 11.16 & 10.88 & 10.22 & 10.00 & \\
\hline 24 & 9.38 & 9.86 & 9.46 & 9.11 & 8.92 & 9.75 & 9.76 & 11.46 & 10.91 & 10.17 & 9.98 & \\
\hline 25 & 9.41 & 9.81 & 9.44 & 9.10 & 8.93 & 9.78 & 9.80 & 11.58 & 10.90 & 10.15 & 10.01 & \\
\hline 26 & 9.38 & 9.79 & 9.43 & 9.09 & 8.96 & 9.93 & 9.81 & 11.61 & 10.88 & 10.12 & 9.97 & \\
\hline 27 & 9.35 & 9.75 & 9.42 & 9.08 & 9.00 & 9.96 & 9.78 & 11.60 & 10.86 & 10.10 & 10.00 & \\
\hline 28 & 9.33 & 9.72 & 9.44 & 9.07 & 9.04 & 10.01 & 9.77 & 11.56 & 10.87 & 10.08 & 9.99 & \\
\hline 29 & 9.31 & 9.64 & 9.44 & 9.05 & 9.09 & 10.05 & 9.77 & 11.52 & 10.85 & 10.05 & 9.97 & \\
\hline 30 & 9.29 & 9.61 & 9.42 & 9.03 & -- & 10.06 & 9.76 & 11.54 & 10.82 & 10.17 & 9.95 & \\
\hline 31 & 9.30 & --- & 9.41 & 9.02 & -- & 10.02 & - & 11.60 & -- & 10.24 & 9.97 & \\
\hline MEAN & 9.53 & 9.80 & 9.49 & 9.20 & 8.96 & 9.70 & 9.76 & 10.36 & 11.13 & 10.52 & 10.18 & \\
\hline MAX & 9.72 & 10.07 & 9.59 & 9.39 & 9.09 & 10.06 & 9.99 & 11.61 & 11.57 & 11.03 & 10.49 & 10. \\
\hline MIN & 9.29 & 9.26 & 9.39 & 9.02 & 8.87 & 9.16 & 9.61 & 9.69 & 10.82 & 10.05 & 9.95 & \\
\hline
\end{tabular}




\section{MIDDLE LAKE AT LAUDERDALE, WI}

LOCATION.--Lat 42 $46^{\prime} 21^{\prime \prime}$, long $88^{\circ} 33^{\prime} 55^{\prime \prime}$, in SE 1/4 SE 1/4 sec.26, T.4 N., R.16 E., Walworth County, Hydrologic Unit 07120006, at Lauderdale. PERIOD OF RECORD.--November 1993 to November 1994, February 1999 to current year.

REMARKS.--Lake sampled near east end of lake at a depth of about $52 \mathrm{ft}$. Lake ice-covered during February sampling. Water-quality analyses done by Wisconsin State Laboratory of Hygiene.

WATER-QUALITY DATA, FEBRUARY 18 TO AUGUST 16, 2004

(Milligrams per liter unless otherwise indicated)

Date

Secchi depth (m)

Depth of sample $(\mathrm{m})$

Chlorophyll a, phytoplankton ( $\mu \mathrm{g} / \mathrm{L}$ )

Water temperature $\left({ }^{\circ} \mathrm{C}\right)$

Specific conductance $(\mu \mathrm{S} / \mathrm{cm})$

$\mathrm{pH}$

Dissolved oxygen (mg/L)

Phosphorus, total (as P)

Phosphorus, ortho, dissolved (as $P$ )

Nitrogen, $\mathrm{NO}_{2}+\mathrm{NO}_{3}$, diss. (as $\mathrm{N}$ )

Nitrogen, ammonia, dissolved (as $\mathrm{N}$ )

Nitrogen, amm. + diss., total (as N)

Nitrogen, amm. + org., total (as N)

Nitrogen, total (as $\mathrm{N}$ )

Color (Pt-Co. scale)

Turbidity (NTU)

Hardness, as $\mathrm{CaCO}_{3}$

Calcium, dissolved (Ca)

Magnesium, dissolved (Mg)

Sodium, dissolved $(\mathrm{Na})$

Potassium, dissolved (K)

Alkalinity, as $\mathrm{CaCO}_{3}$

Sulfate, dissolved $\left(\mathrm{SO}_{4}\right)$

Chloride, dissolved (Cl)

Silica, dissolved $\left(\mathrm{SiO}_{2}\right)$

Solids, dissolved, at $180^{\circ} \mathrm{C}$

Iron, dissolved ( $\mathrm{Fe}$ ) ( $\mu \mathrm{g} / \mathrm{L}$ )

Manganese, dissolved, $(\mathrm{Mn})(\mu \mathrm{g} / \mathrm{L})$

\begin{tabular}{|c|c|c|c|c|c|c|c|c|c|}
\hline \multicolumn{2}{|c|}{ Feb-18 } & \multicolumn{2}{|c|}{ Apr-8 } & \multicolumn{2}{|c|}{ Jun-7 } & \multicolumn{2}{|c|}{$\underline{\mathrm{Jul}}-12$} & \multicolumn{2}{|c|}{ Aug-16 } \\
\hline- & - & & & & & & & & \\
\hline 0.5 & 13.5 & 0.5 & 14 & 0.5 & 14 & 0.5 & 14 & 0.5 & 14 \\
\hline- & - & 2.0 & - & 3.7 & - & 6.7 & - & 4.8 & - \\
\hline 0.6 & 4.5 & 10.1 & 4.9 & 21.6 & 6.5 & 23.9 & 7.1 & 21.5 & 7.5 \\
\hline 540 & 662 & 521 & 619 & 491 & 597 & 477 & 604 & 502 & 622 \\
\hline 8.0 & 7.6 & 8.3 & 7.6 & 8.0 & 7.2 & 8.0 & 7.4 & 8.0 & 7.1 \\
\hline 15.1 & 8.4 & 11.9 & 7.6 & 9.3 & 0.5 & 11.2 & 0.3 & 8.7 & 0.3 \\
\hline 0.011 & 0.012 & 0.015 & 0.021 & 0.047 & 0.024 & 0.013 & 0.035 & 0.016 & 0.044 \\
\hline- & - & 0.001 & - & - & - & $<0.002$ & - & - & - \\
\hline- & - & 0.868 & - & -- & - & 0.309 & - & - & - \\
\hline- & -- & 0.223 & - & - & - & 0.08 & - & - & - \\
\hline - & - & -- & - & - & - & 0.65 & - & - & - \\
\hline- & - & 0.89 & - & -- & - & - & -- & - & - \\
\hline- & - & 1.8 & -- & - & -- & - & - & - & - \\
\hline- & - & 5 & - & - & - & - & - & - & - \\
\hline- & - & 1.2 & - & -- &.- & - & - & - & - \\
\hline- & - & 250 & - & - & - & - & - & - & - \\
\hline- & - & 44.2 & - & - & -- & - & - & - & - \\
\hline- & -- & 34.1 & - & - & -- & - & - & - & - \\
\hline- & - & 8.8 & - & - & -- & -- & -- & - & - \\
\hline- & - & 2 & - & -- & - & - & -- & - & - \\
\hline- & -- & 202 & - & - & - & - & - & - & - \\
\hline- & - & 36.1 & - & - & -- & - & - & - & - \\
\hline- & - & 24.3 & - & - & -- & - & - & - & - \\
\hline- & - & 2.85 & - & - & - & - & - & - & - \\
\hline- & - & 292 & - & - & - & - & - & -- & - \\
\hline- & -- & $<100$ & -- & - & - & - & - & - & - \\
\hline- & - & $<1$ & -- & - & -- & - & -- & - & - \\
\hline
\end{tabular}

7-12-04

DISSOLVED OXYGEN (D.O.) IN MILLIGRAMS PER LITER

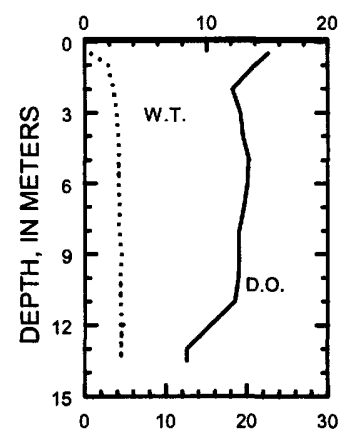

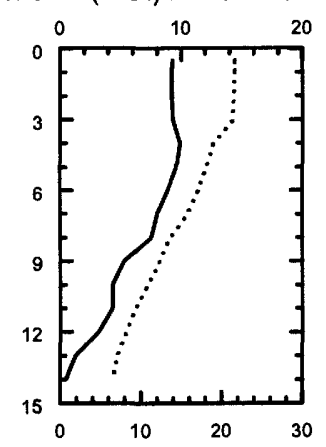
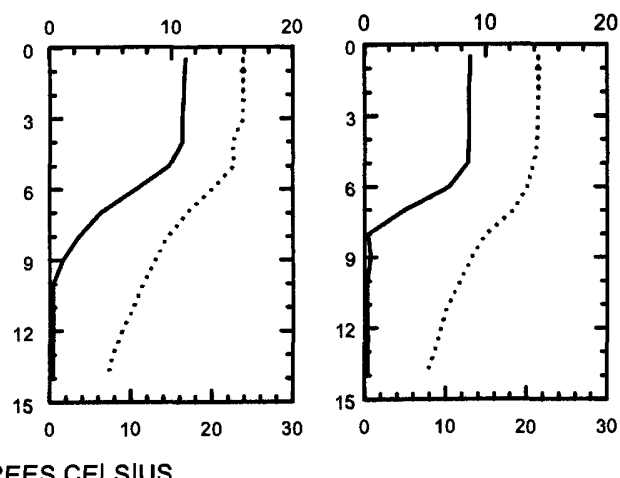

WATER TEMPERATURE (W.T.) IN DEGREES CELSIUS
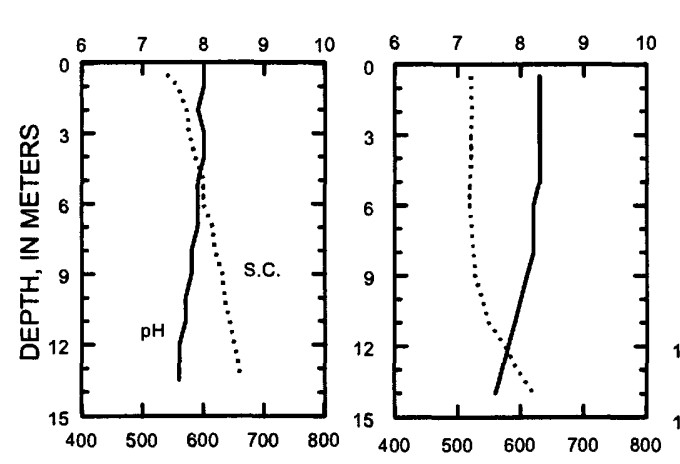

PH IN STANDARD UNITS
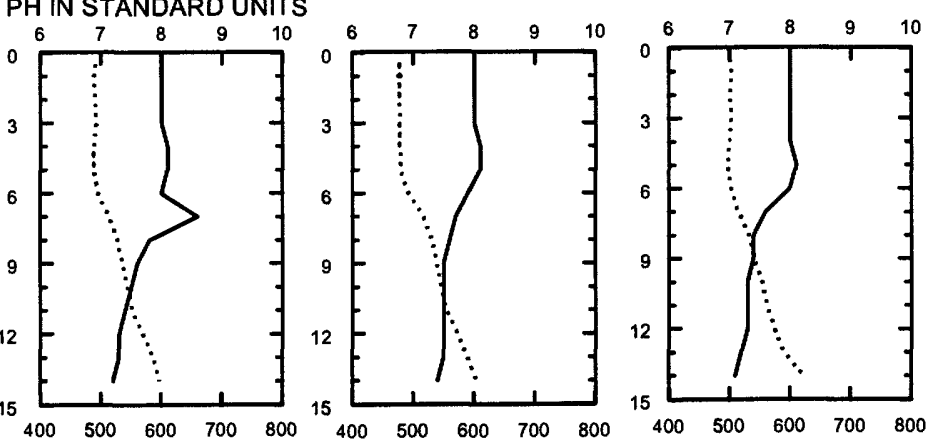

SPECIFIC CONDUCTANCE (S.C.) IN MICROSIEMENS PER CENTIMETER AT 25 DEGREES CELSIUS 

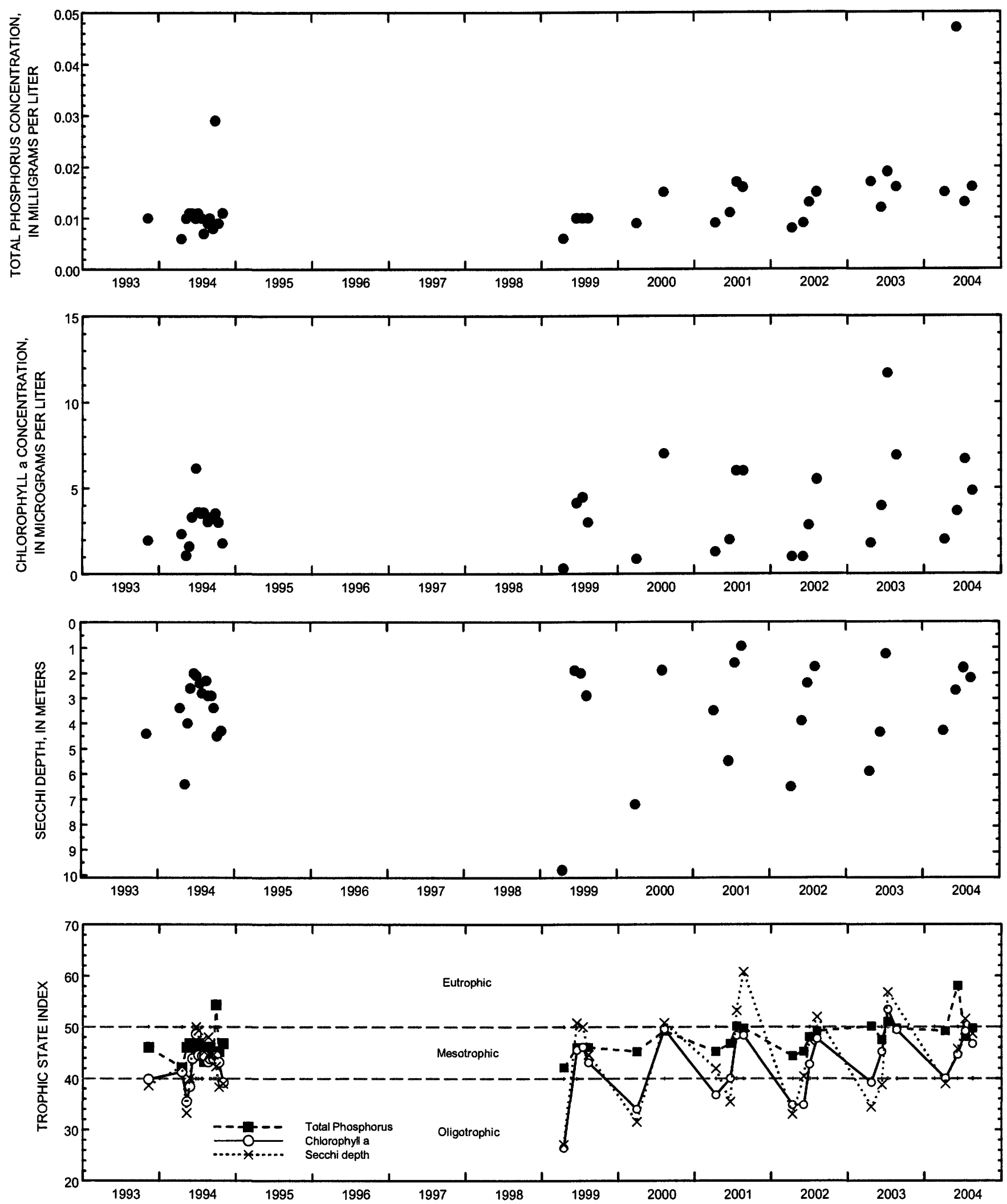

Surface total phosphorus, chlorophyll a concentrations, Secchi depths, and TSI data for Middle Lake, near Lauderdale, Wisconsin. 


\section{MIDDLE GENESEE LAKE NEAR OCONOMOWOC, WI}

LOCATION.--Lat $43^{\circ} 02^{\prime} 51^{\prime \prime}$, long 88²8'47", in SW 1/4 SW 1/4 SW 1/4 sec.22, T. 7 N., R.17 E., Waukesha County, Hydrologic Unit 07090001, at the southwest side of the lake about 2 miles south of Oconomowoc.

DRAINAGE AREA.--Unknown. Area of Middle Genesee Lake is $0.17 \mathrm{mi}^{2}$.

PERIOD OF RECORD.--April 1996 to current year.

GAGE.--Staff gage. Local observer, Tom Schubring provided most readings of gage.

EXTREMES FOR THE PERIOD OF RECORD.--Maximum observed gage height, $867.18 \mathrm{ft}$, June 13, 2001 ; minimum observed, $863.92 \mathrm{ft}$, Oct. 22, 31 and Nov. 1, 2003.

EXTREMES FOR CURRENT YEAR.--Maximum observed gage height, $867.14 \mathrm{ft}$, June 22; minimum observed, $863.92 \mathrm{ft}$, Oct. 22,31 and Nov. 1. GAGE HEIGHT, FEET, WATER YEAR OCTOBER 2003 TO OCTOBER 2004

DAILY MEAN VALUES

$\begin{array}{rcrc}\text { Date } & \begin{array}{c}\text { Gage height } \\ \text { (feet) }\end{array} & \text { Date } & \begin{array}{c}\text { Gage height } \\ \text { (feet) }\end{array} \\ \text { October 1 } & 864.08 & 19 & 864.34 \\ 3 & 864.06 & 29 & 864.34 \\ 8 & 864.02 & \text { December 2 } & 864.32 \\ 11 & 864.00 & 8 & 864.32 \\ 18 & 863.98 & \text { April 14 } & 864.86 \\ 22 & 863.92 & 22 & 865.20 \\ 26 & 863.98 & \text { May 2 } & 865.22 \\ 29 & 863.94 & 9 & 865.26 \\ 31 & 863.92 & 10 & 865.40 \\ \text { November 1 } & 863.92 & 26 & 866.52 \\ 4 & 864.28 & 29 & 866.62 \\ 5 & 864.30 & 31 & 866.76 \\ 14 & 864.26 & \text { June 8 } & 867.00 \\ 17 & 864.26 & 22 & 867.14\end{array}$

$\begin{array}{rc}\text { Date } & \begin{array}{c}\text { Gage height } \\ \text { (feet) }\end{array} \\ \text { June } 24 & 867.12 \\ 27 & 867.09 \\ \text { July } 4 & 867.06 \\ 9 & 867.02 \\ 12 & 867.07 \\ 15 & 867.02 \\ 18 & 867.02 \\ \text { July } 26 & 866.90 \\ 29 & 866.88 \\ 31 & 866.84 \\ \text { August } 1 & 866.82 \\ 3 & 866.84 \\ 6 & 867.04 \\ 8 & 867.00\end{array}$

$\begin{array}{rc}\text { Date } & \begin{array}{c}\text { Gage height } \\ \text { (feet) }\end{array} \\ \text { August 14 } & 866.90 \\ 20 & 866.84 \\ 31 & 866.86 \\ \text { September 6 } & 866.78 \\ 12 & 866.68 \\ 18 & 866.58 \\ 26 & 866.44 \\ 30 & 866.36 \\ \text { October 4 } & 866.26 \\ 15 & 866.08 \\ 24 & 865.94 \\ 28 & 865.86 \\ 31 & 865.82\end{array}$

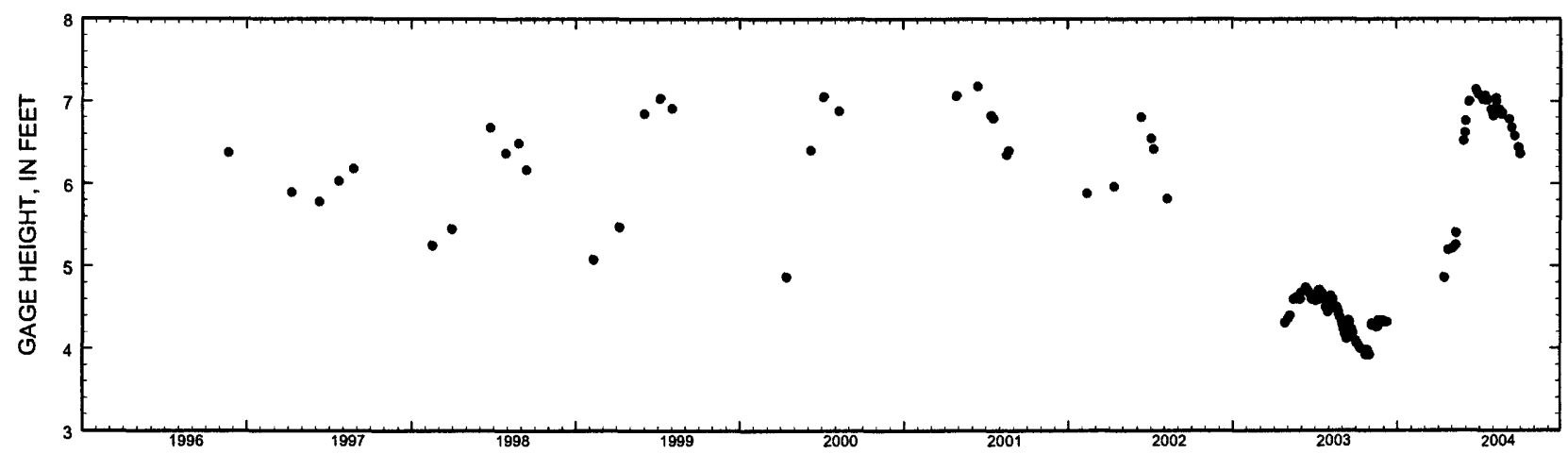




\section{MIDDLE GENESEE LAKE NEAR OCONOMOWOC, WI}

LOCATION.--Lat $43^{\circ} 03^{\prime} 09^{\prime \prime}$, long $88^{\circ} 28^{\prime} 48^{\prime \prime}$, in NW 1/4 SW 1/4 sec.22, T.7 N., R.17 E., Waukesha County, Hydrologic Unit 07090001 , 1.8 mi south of Oconomowoc.

PERIOD OF RECORD.--February 1996 to current year.

REMARKS.--Lake sampled near center at the deep hole. Lake ice-covered during February sampling. Water-quality analyses done by Wisconsin State Laboratory of Hygiene.

WATER-QUALITY DATA, FEBRUARY 19 TO AUGUST 3, 2004

(Milligrams per liter unless otherwise indicated)

Date

Lake stage $(\mathrm{ft})$

Secchi depth $(\mathrm{m})$

Depth of sample $(\mathrm{m})$

Chlorophyll a, phytoplankton ( $\mu \mathrm{g} / \mathrm{L})$

Water temperature $\left({ }^{\circ} \mathrm{C}\right)$

Specific conductance $(\mu \mathrm{S} / \mathrm{cm})$

$\mathrm{pH}$

Dissolved oxygen (mg/L)

Phosphorus, total (as P)

Phosphorus, ortho, dissolved (as P)

Nitrogen, $\mathrm{NO}_{2}+\mathrm{NO}_{3}$, diss. (as $\mathrm{N}$ )

Nitrogen, ammonia, dissolved (as N)

Nitrogen, amm. + org., total (as N)

Nitrogen, total (as N)

Color (Pt-Co. scale)

Turbidity (NTU)

Hardness, as $\mathrm{CaCO}_{3}$

Calcium, dissolved ( $\mathrm{Ca}$ )

Magnesium, dissolved $(\mathrm{Mg})$

Sodium, dissolved $(\mathrm{Na})$

Potassium, dissolved $(\mathrm{K})$

Alkalinity, as $\mathrm{CaCO}_{3}$

Sulfate, dissolved $\left(\mathrm{SO}_{4}\right)$

Chloride, dissolved $(\mathrm{Cl})$

Silica, dissolved $\left(\mathrm{SiO}_{2}\right)$

Solids, dissolved, at $180^{\circ} \mathrm{C}$

Iron, dissolved (Fe) ( $\mu \mathrm{g} / \mathrm{L})$

Manganese, dissolved, $(\mathrm{Mn})(\mu \mathrm{g} / \mathrm{L})$

2-19-04

\begin{tabular}{cccccc}
\multicolumn{2}{c}{ Feb-19 } & \multicolumn{2}{c}{ Apr-14 } & \multicolumn{2}{c}{$\frac{\text { Jun-8 }}{867.00}$} \\
& - & \multicolumn{2}{c}{864.86} & \multicolumn{2}{c}{3.4} \\
0.5 & 11.5 & 0.5 & 11.5 & 0.5 & 12 \\
- & - & 0.8 & - & 3.7 & - \\
2.7 & 5.4 & 9.4 & 8.3 & 22.5 & 12.9 \\
477 & 555 & 421 & 420 & 418 & 45 \\
8.0 & 7.2 & 8.2 & 8.2 & 8.2 & 7.3 \\
13.4 & 0.9 & 11.8 & 11.3 & 9.9 & 0.4 \\
0.009 & 0.017 & 0.017 & 0.014 & 0.015 & 0.023
\end{tabular}

$\begin{array}{lllll}0.017 & 0.014 & 0.015 & 0.023 & 0.021\end{array}$

$\begin{array}{llll}0.021 & 0.061 & 0.010 & 0.060\end{array}$

\begin{tabular}{cccc}
\multicolumn{2}{c}{$\frac{\mathrm{Jul}-15}{876.02}$} & \multicolumn{2}{c}{$\frac{\mathrm{Aug}-3}{866.84}$} \\
\multicolumn{2}{c}{3.5} & & \multicolumn{2}{c}{3.2} \\
0.5 & 11.5 & 0.5 & 11.5 \\
1.5 &.- & 2.5 & - \\
24.6 & 12.8 & 26.3 & 12.7 \\
428 & 489 & 433 & 516 \\
8.0 & 7.2 & 8.3 & 7.3 \\
9.5 & 0.2 & 9.3 & 0.3 \\
0.021 & 0.061 & 0.010 & 0.060
\end{tabular}

$\begin{array}{lll}- & - & 0.042 \\ - & - & 0.197\end{array}$

$\begin{array}{lll}- & - & 0.93\end{array}$

$-$

$-$

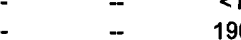

$-$

- $\quad-\quad-27.6$

$\begin{array}{lll}- & - & 13.1\end{array}$

- $\quad-\quad 1$

$\begin{array}{lll}- & - & 160\end{array}$

$-\quad-\quad-14.8$

$\begin{array}{lll}- & - & 28.9 \\ - & - & 1.14\end{array}$

- $\quad--\quad 236$

$-$

4-14-04

6-8-04

7-15-04

DISSOLVED OXYGEN (D.O.) IN MILLIGRAMS PER LITER
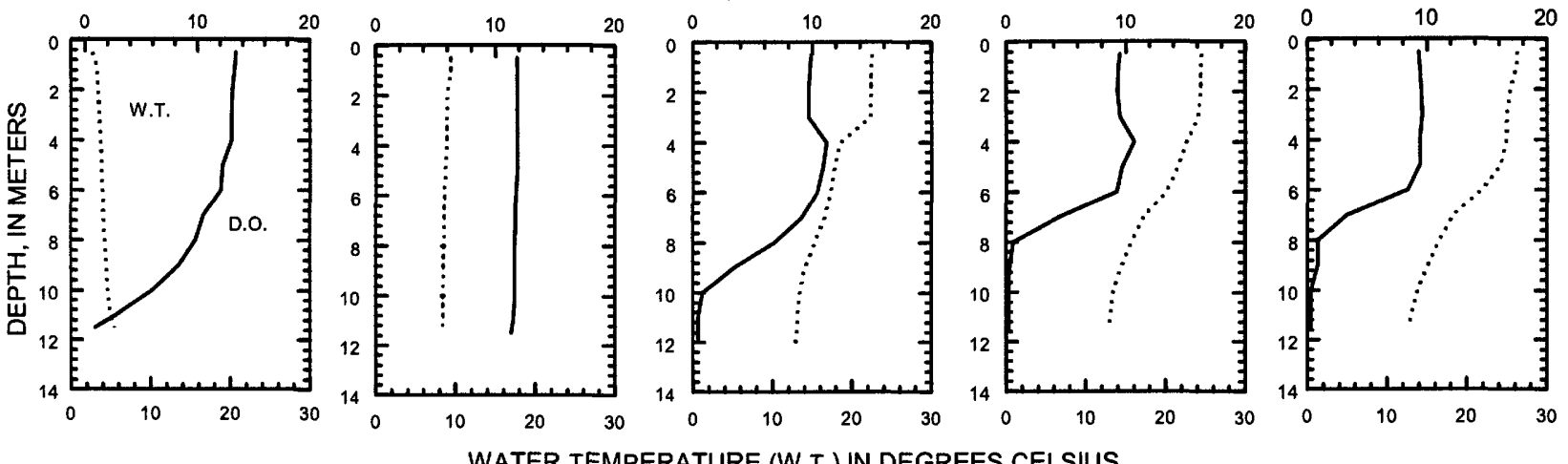

WATER TEMPERATURE (W.T.) IN DEGREES CELSIUS

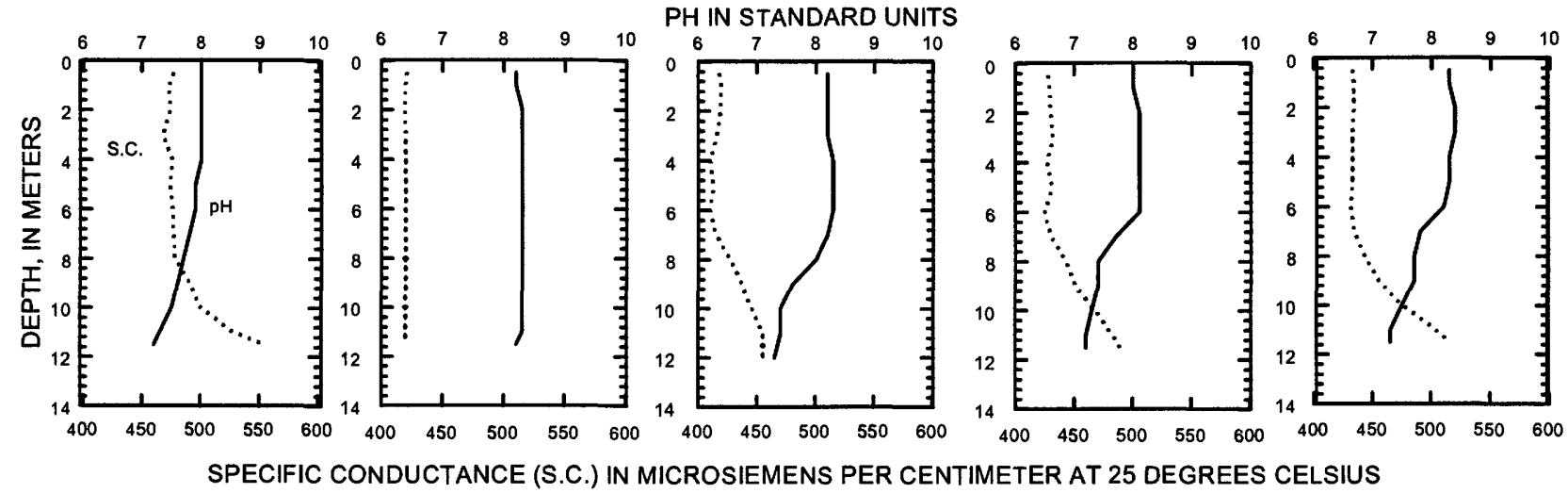



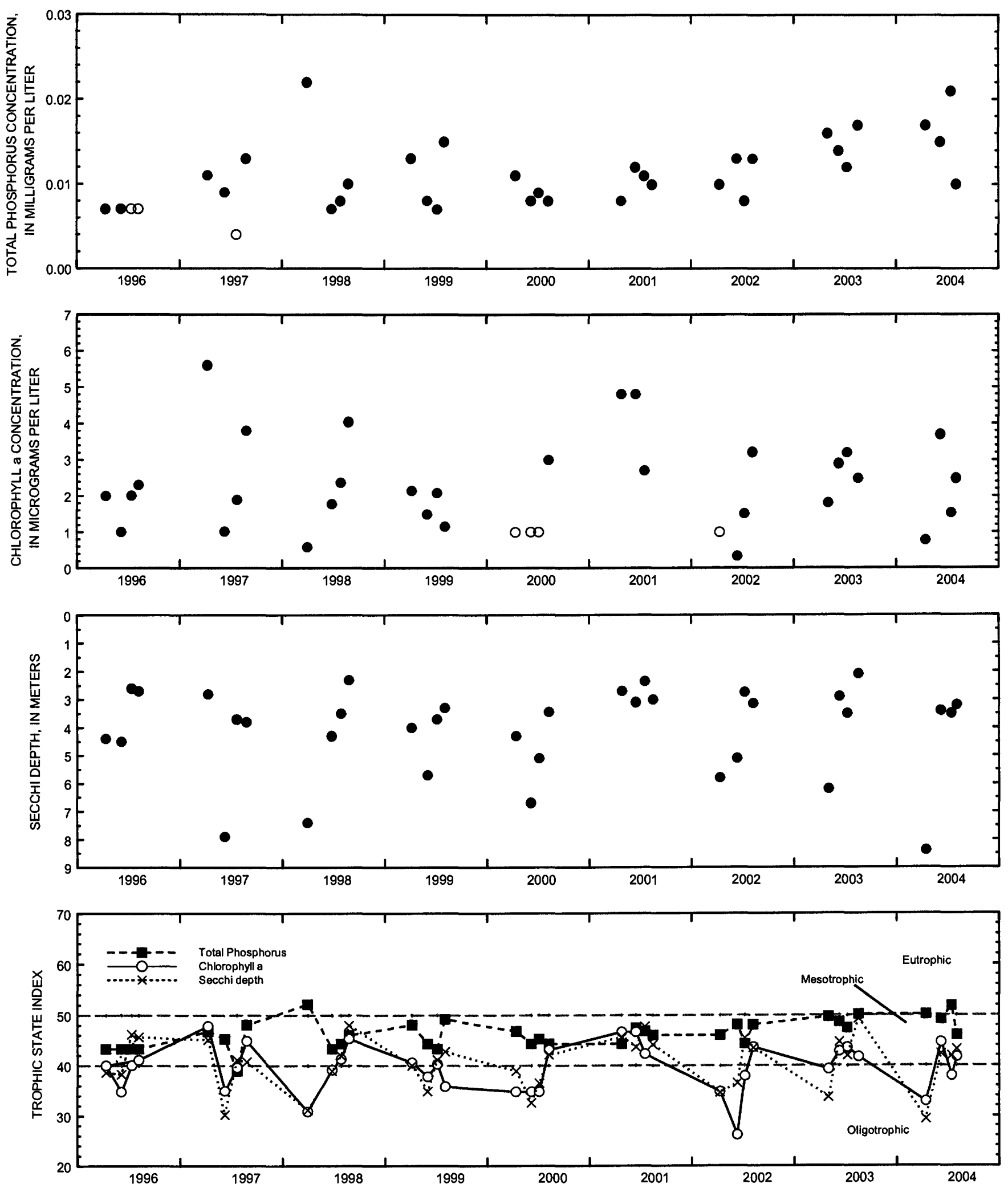

Surface total phosphorus, chlorophyll a concentrations, Secchi depths, and TSI data for Middle Genesee Lake, near Oconomowoc, Wisconsin.

(Circles on the first two plots indicate laboratory detection limit for selected analyses. Actual concentrations for these particular analyses are less than the plotted circles.) 
LOCATION.--Lat 4303'48", long 89²3'49', in SE 1/4 SW 1/4 sec.23, T.7 N., R.9 E., Dane County, Hydrologic Unit 07090001, in Brittingham Park, in Madison.

DRAINAGE AREA.--279 $\mathrm{mi}^{2}$. Area of Lake Monona, $5.3 \mathrm{mi}^{2}$.

PERIOD OF RECORD.--September 1915 to current year (fragmentary) in reports of the Geological Survey. For 1856 to March 1917 in reports of Wisconsin Railroad Commission, volume 19.

REVISED RECORDS.--WSP 1338: Lake area. WDR WI-73-1: Drainage area.

GAGE.--Water-stage recorder. Datum of gage is $840.00 \mathrm{ft}$ above sea level, or $5.60 \mathrm{ft}$ below City of Madison datum. Prior to Oct. 1, 1979 , datum $3.61 \mathrm{ft}$ higher; prior to Nov. 15, 1971, nonrecording gage at same site at the higher datum.

REMARKS.--Lake level regulated by concrete dam with four 12-foot stop-log sections and 12-foot lock at outlet of Lake Waubesa. Gageheight telemeter at station.

EXTREMES FOR PERIOD OF RECORD.--Maximum gage height observed, $7.48 \mathrm{ft}$, June 14, 15, 2000; minimum observed, $3.22 \mathrm{ft}$, Jan. 20 , 1965 , current datum.

EXTREMES FOR CURRENT YEAR.--Maximum recorded gage height, $6.87 \mathrm{ft}$, June 11; minimum recorded, 4.10, Feb. $17,18,19$.

\section{WATER YEAR OCTOBER 2003 TO SEPTEMBER 2004 DAILY MEAN VALUES}

\begin{tabular}{|c|c|c|c|c|c|c|c|c|c|c|c|}
\hline DAY & OCT & NOV & DEC & JAN & FEB & MAR & APR & MAY & JUN & JUL & AUG \\
\hline 1 & 4.84 & 4.52 & 5.39 & 4.48 & 4.15 & 4.19 & 4.91 & 4.68 & 6.75 & 6.31 & 6.07 \\
\hline 2 & 4.83 & 4.70 & 5.38 & 4.47 & 4.15 & 4.19 & 4.94 & 4.68 & 6.75 & 6.34 & 6.02 \\
\hline 3 & 4.81 & 4.91 & 5.37 & 4.45 & 4.16 & 4.18 & 4.93 & 4.68 & 6.73 & 6.40 & 6.01 \\
\hline 4 & 4.79 & 5.36 & 5.28 & 4.43 & 4.16 & 4.18 & 4.94 & 4.68 & 6.73 & 6.61 & 6.17 \\
\hline 5 & 4.79 & 5.47 & 5.21 & 4.43 & 4.15 & 4.38 & 4.97 & 4.68 & 6.73 & 6.62 & 6.15 \\
\hline 6 & 4.78 & 5.40 & 5.14 & 4.43 & 4.17 & 4.45 & 4.99 & 4.69 & 6.73 & 6.58 & 6.11 \\
\hline 7 & 4.77 & 5.30 & 5.10 & 4.42 & 4.17 & 4.45 & 5.01 & 4.72 & 6.73 & 6.54 & 6.08 \\
\hline 8 & 4.76 & 5.23 & 5.06 & 4.40 & 4.17 & 4.42 & 5.00 & 4.71 & 6.73 & 6.50 & 6.07 \\
\hline 9 & 4.75 & 5.22 & 5.02 & 4.39 & 4.16 & 4.41 & 5.01 & 4.82 & 6.74 & 6.52 & 6.04 \\
\hline 10 & 4.75 & 5.23 & 5.15 & 4.37 & 4.15 & 4.41 & 5.01 & 4.89 & 6.76 & 6.53 & 6.01 \\
\hline 11 & 4.75 & 5.22 & 5.16 & 4.36 & 4.15 & 4.39 & 5.01 & 4.99 & 6.87 & 6.51 & 5.97 \\
\hline 12 & 4.74 & 5.17 & 5.14 & 4.34 & 4.13 & 4.36 & 5.01 & 5.04 & 6.83 & 6.50 & 5.94 \\
\hline 13 & 4.74 & 5.12 & 5.12 & 4.31 & 4.13 & 4.36 & 4.96 & 5.10 & 6.76 & 6.46 & 5.93 \\
\hline 14 & 4.81 & 5.12 & 5.08 & 4.29 & 4.12 & 4.35 & 4.94 & 5.13 & 6.70 & 6.43 & 5.92 \\
\hline 15 & 4.80 & 5.12 & 5.04 & 4.28 & 4.11 & 4.34 & 4.92 & 5.15 & 6.65 & 6.42 & 5.91 \\
\hline 16 & 4.79 & 5.12 & 4.98 & 4.26 & 4.11 & 4.33 & 4.88 & 5.15 & 6.63 & 6.47 & 5.89 \\
\hline 17 & 4.77 & 5.12 & 4.93 & 4.27 & 4.10 & 4.34 & 4.91 & 5.15 & 6.72 & 6.58 & 5.90 \\
\hline 18 & 4.76 & 5.16 & 4.89 & 4.25 & 4.10 & 4.34 & 4.89 & 5.26 & 6.72 & 6.56 & 5.89 \\
\hline 19 & 4.76 & 5.13 & 4.84 & 4.23 & 4.10 & 4.34 & 4.87 & 5.24 & 6.71 & 6.52 & 5.91 \\
\hline 20 & 4.74 & 5.16 & 4.79 & 4.22 & 4.14 & 4.31 & 4.87 & 5.20 & 6.68 & 6.49 & 5.88 \\
\hline 21 & 4.71 & 5.20 & 4.75 & 4.22 & 4.17 & 4.29 & 4.88 & 5.29 & 6.68 & 6.48 & 5.84 \\
\hline 22 & 4.69 & 5.23 & 4.72 & 4.20 & 4.19 & 4.30 & 4.87 & 5.86 & 6.66 & 6.47 & 5.82 \\
\hline 23 & 4.65 & 5.39 & 4.69 & 4.20 & 4.21 & 4.32 & 4.84 & 6.40 & 6.63 & 6.45 & 5.81 \\
\hline 24 & 4.65 & 5.45 & 4.65 & 4.20 & 4.23 & 4.35 & 4.82 & 6.56 & 6.59 & 6.39 & 5.78 \\
\hline 25 & 4.69 & 5.47 & 4.62 & 4.19 & 4.23 & 4.38 & 4.81 & 6.61 & 6.55 & 6.33 & 5.79 \\
\hline 26 & 4.65 & 5.48 & 4.60 & 4.19 & 4.22 & 4.59 & 4.76 & 6.60 & 6.49 & 6.27 & 5.79 \\
\hline 27 & 4.62 & 5.48 & 4.58 & 4.19 & 4.20 & 4.67 & 4.73 & 6.57 & 6.44 & 6.21 & 5.81 \\
\hline 28 & 4.59 & 5.44 & 4.58 & 4.18 & 4.19 & 4.71 & 4.72 & 6.56 & 6.40 & 6.11 & 5.83 \\
\hline 29 & 4.57 & 5.44 & 4.55 & 4.17 & 4.18 & 4.76 & 4.71 & 6.60 & 6.32 & 6.04 & 5.83 \\
\hline 30 & 4.56 & 5.42 & 4.53 & 4.16 & - & 4.81 & 4.70 & 6.67 & 6.29 & 6.10 & 5.82 \\
\hline 31 & 4.52 & -- & 4.50 & 4.16 & - & 4.87 & - & 6.72 & - & 6.12 & 5.81 \\
\hline MEAN & 4.72 & 5.23 & 4.93 & 4.29 & 4.16 & 4.41 & 4.89 & 5.45 & 6.66 & 6.41 & 5.93 \\
\hline MAX & 4.84 & 5.48 & 5.39 & 4.48 & 4.23 & 4.87 & 5.01 & 6.72 & 6.87 & 6.62 & 6.17 \\
\hline MIN & 4.52 & 4.52 & 4.50 & 4.16 & 4.10 & 4.18 & 4.70 & 4.68 & 6.29 & 6.04 & 5.78 \\
\hline
\end{tabular}




\section{MUSKEGO (BIG MUSKEGO) LAKE NEAR WIND LAKE, WI}

LOCATION.--Lat $42^{\circ} 51^{\prime} 09^{\prime \prime}$, long $88^{\circ} 07^{\prime} 50^{\prime \prime}$, in SE $1 / 4$ NE 1/4 sec.33, T.5 N., R.20 E., Waukesha County, Hydrologic Unit 07120006, on left bank $8 \mathrm{ft}$ upstream of dam outlet of Muskego Lake, $700 \mathrm{ft}$ north of Muskego Dam Drive, 2 mi northeast of Wind Lake.

DRAINAGE AREA.--33.9 $\mathrm{mi}^{2}$ (revised).

PERIOD OF RECORD.--October 1987 to September 1989, January 1991 to current year. Prior to October 1993, published as Muskego Lake Outlet near Wind Lake, WI. October 1993 to September 2000, published as "Big Muskego Lake".

GAGE.--Water-stage recorder. Datum of gage is $760.00 \mathrm{ft}$ above sea level. October to December 1987 and January 1991 to September 1995 , nonrecording gage at the same datum. December 1987 through September 1989, data collected using water-stage recorder at the same datum.

REMARKS.--Lake levels regulated by concrete dam with one 5-ft lift gate.

EXTREMES FOR PERIOD OF RECORD.--Maximum observed gage height, $12.60 \mathrm{ft}$, Oct. 7, 1991 and Aug. 8, 1994; minimum instantaneous, less than $8.72 \mathrm{ft}$, July 12,1996 to Feb. 18, 1997, due to drawdown of lake.

EXTREMES FOR CURRENT YEAR.--Maximum observed gage-height, $12.25 \mathrm{ft}$, May 25; minimum observed, $10.58 \mathrm{ft}$, Oct. 20.

GAGE HEIGHT, FEET

WATER YEAR OCTOBER 2003 TO SEPTEMBER 2004

DAILY MEAN VALUES

\begin{tabular}{|c|c|c|c|c|c|c|c|c|c|c|c|c|}
\hline DAY & OCT & NOV & DEC & JAN & FEB & MAR & APR & MAY & JUN & JUL & AUG & SEP \\
\hline 1 & 10.71 & 10.99 & 11.58 & 11.70 & 11.69 & 11.73 & 11.82 & e11.44 & 11.96 & 11.47 & 11.00 & 11.09 \\
\hline 2 & 10.67 & 11.08 & 11.60 & 11.70 & 11.69 & 11.77 & 11.80 & e11.42 & 11.97 & 11.46 & 11.00 & 11.08 \\
\hline 3 & 10.61 & 11.25 & 11.60 & 11.70 & 11.70 & 11.78 & 11.84 & e11.42 & 11.90 & 11.44 & 11.03 & 11.09 \\
\hline 4 & 10.69 & 11.28 & 11.61 & 11.70 & 11.70 & 11.81 & 11.76 & e11.38 & 11.82 & 11.57 & 11.11 & 11.08 \\
\hline 5 & 10.69 & 11.33 & 11.61 & 11.70 & 11.69 & 11.94 & 11.72 & e11.32 & 11.74 & 11.64 & 11.11 & 11.07 \\
\hline 6 & 10.67 & 11.34 & 11.61 & 11.70 & 11.71 & 11.97 & 11.71 & e11.28 & 11.67 & 11.61 & 11.07 & 11.02 \\
\hline 7 & 10.64 & 11.36 & 11.61 & 11.69 & 11.70 & 11.95 & 11.70 & 11.23 & 11.63 & 11.69 & 11.06 & 11.08 \\
\hline 8 & 10.64 & 11.40 & 11.62 & 11.69 & 11.70 & 11.93 & 11.63 & 11.10 & 11.62 & 11.64 & 11.04 & 11.10 \\
\hline 9 & 10.66 & 11.40 & 11.64 & 11.69 & 11.69 & 11.89 & 11.55 & 11.15 & 11.62 & 11.54 & 11.02 & 11.06 \\
\hline 10 & 10.66 & 11.40 & 11.80 & 11.69 & 11.68 & 11.82 & 11.50 & 11.16 & 11.62 & 11.50 & 11.02 & 11.03 \\
\hline 11 & 10.62 & 11.40 & 11.77 & 11.69 & 11.68 & 11.78 & 11.44 & 11.21 & 11.65 & 11.43 & 11.02 & 11.01 \\
\hline 12 & 10.63 & 11.32 & 11.77 & 11.68 & 11.67 & 11.77 & 11.41 & 11.15 & 11.65 & 11.40 & 11.04 & 11.01 \\
\hline 13 & 10.61 & 11.41 & 11.77 & 11.68 & 11.67 & 11.73 & 11.37 & 11.30 & 11.62 & 11.31 & 11.03 & 11.00 \\
\hline 14 & 10.71 & 11.38 & 11.77 & 11.68 & 11.66 & 11.64 & 11.27 & 11.49 & 11.62 & 11.25 & 11.02 & 10.97 \\
\hline 15 & 10.65 & 11.41 & 11.76 & 11.68 & 11.65 & 11.71 & 11.19 & 11.60 & 11.64 & 11.20 & 11.00 & 10.89 \\
\hline 16 & 10.67 & 11.43 & 11.75 & 11.68 & 11.65 & 11.65 & 11.16 & 11.60 & 11.61 & 11.25 & 10.97 & 10.99 \\
\hline 17 & 10.63 & 11.44 & 11.75 & 11.69 & 11.64 & 11.57 & 11.25 & 11.56 & 11.63 & 11.32 & 10.99 & 11.00 \\
\hline 18 & 10.61 & 11.53 & 11.74 & 11.69 & 11.64 & 11.56 & 11.01 & 11.70 & 11.62 & 11.26 & 10.95 & 10.97 \\
\hline 19 & 10.65 & 11.55 & 11.73 & 11.68 & 11.64 & 11.51 & 11.02 & 11.69 & 11.61 & 11.22 & 10.99 & 10.94 \\
\hline 20 & 10.58 & 11.53 & 11.72 & 11.68 & 11.65 & 11.51 & 11.17 & 11.68 & 11.53 & 11.21 & 10.98 & 10.93 \\
\hline 21 & 10.67 & 11.64 & 11.71 & 11.68 & 11.66 & 11.52 & 11.18 & 11.73 & 11.55 & 11.20 & 10.97 & 10.91 \\
\hline 22 & 10.69 & 11.63 & 11.71 & 11.68 & 11.65 & 11.41 & 11.34 & 11.85 & 11.59 & 11.21 & 10.89 & 10.92 \\
\hline 23 & 10.70 & 11.53 & 11.70 & 11.69 & 11.66 & 11.42 & 11.28 & 12.08 & 11.56 & 11.20 & 10.97 & 10.90 \\
\hline 24 & 10.73 & 11.49 & 11.70 & 11.70 & 11.67 & 11.43 & 11.51 & 12.23 & 11.57 & 11.15 & 10.94 & 10.85 \\
\hline 25 & 10.82 & 11.58 & 11.69 & 11.69 & 11.67 & 11.43 & 11.49 & 12.25 & 11.54 & 11.14 & 11.02 & 10.89 \\
\hline 26 & 10.85 & 11.60 & 11.69 & 11.70 & 11.67 & 11.59 & e11.46 & 12.21 & 11.52 & 11.09 & 11.01 & 10.88 \\
\hline 27 & 10.87 & 11.61 & 11.69 & 11.70 & 11.68 & 11.65 & e11.44 & 12.15 & 11.49 & 11.07 & 11.03 & 10.87 \\
\hline 28 & 10.83 & 11.61 & 11.70 & 11.70 & 11.68 & 11.63 & e11.42 & 12.09 & 11.49 & 11.05 & 11.11 & 11.00 \\
\hline 29 & 10.90 & 11.60 & 11.71 & 11.70 & 11.70 & 11.76 & e11.44 & 12.01 & 11.43 & 11.03 & 11.11 & 10.86 \\
\hline 30 & 10.94 & 11.59 & 11.70 & 11.70 & $\cdots$ & 11.89 & e11.44 & 11.99 & 11.44 & 11.03 & 11.09 & 10.83 \\
\hline 31 & 10.94 & $\cdots$ & 11.70 & 11.70 & -- & 11.86 & - & 11.99 & -- & 11.03 & 11.10 & - \\
\hline MEAN & 10.71 & 11.44 & 11.69 & 11.69 & 11.67 & 11.70 & 11.44 & 11.63 & 11.63 & 11.31 & 11.02 & 10.98 \\
\hline MAX & 10.94 & 11.64 & 11.80 & 11.70 & 11.71 & 11.97 & 11.84 & 12.25 & 11.97 & 11.69 & 11.11 & 11.10 \\
\hline MIN & 10.58 & 10.99 & 11.58 & 11.68 & 11.64 & 11.41 & 11.01 & 11.10 & 11.43 & 11.03 & 10.89 & 10.83 \\
\hline
\end{tabular}

e Estimated 
LOCATION.--Lat $43^{\circ} 03^{\prime} 47^{\prime \prime}$, long $88^{\circ} 24^{\prime} 08^{\prime \prime}$, in SW 1/4 SW 1/4 sec.17, T.7 N., R.18 E., Waukesha County, Hydrologic Unit 07090001, on dike of Nagawicka Lake dam about $120 \mathrm{ft}$ west of gates in Delafield.

DRAINAGE AREA.--44.9 $\mathrm{mi}^{2}$. Area of Nagawicka Lake, 917 acres.

PERIOD OF RECORD.--October 2002 to current year.

GAGE.--Water-stage recorder.

REMARKS.--Gage established Oct. 29, 2002. Lake levels controlled by City of Delafield.

EXTREMES FOR CURRENT YEAR.--Maximum gage height, $8.73 \mathrm{ft}$, May 23, affected by wind; minimum gage height, $7.60 \mathrm{ft}$, Dec. 1 .

\section{GAGE HEIGHT, FEET \\ WATER YEAR OCTOBER 2003 TO SEPTEMBER 2004 DAILY MEAN VALUES}

\begin{tabular}{|c|c|c|c|c|c|c|c|c|c|c|c|c|}
\hline DAY & OCT & NOV & DEC & JAN & FEB & MAR & APR & MAY & JUN & JUL & AUG & SEP \\
\hline 1 & 8.28 & 8.35 & 7.64 & 7.92 & 7.80 & 7.84 & 8.27 & 8.38 & 8.41 & 8.42 & 8.34 & 8.37 \\
\hline 2 & 8.27 & 8.44 & 7.67 & 7.92 & 7.79 & 7.89 & 8.24 & 8.38 & 8.47 & 8.42 & 8.34 & 8.3 \\
\hline 3 & 8.28 & 8.55 & 7.70 & 7.92 & 7.80 & 7.93 & 8.23 & 8.36 & 8.49 & 8.40 & 8.40 & 8.3 \\
\hline 4 & 8.30 & 8.61 & 7.72 & 7.93 & 7.79 & 7.97 & 8.20 & 8.34 & 8.48 & 8.40 & 8.56 & 8.3 \\
\hline 5 & 8.32 & 8.51 & 7.74 & 7.92 & 7.79 & 8.12 & 8.18 & 8.35 & 8.45 & 8.41 & 8.42 & 8. \\
\hline 6 & 8.32 & 8.35 & 7.74 & 7.90 & 7.80 & 8.14 & 8.19 & 8.35 & 8.41 & 8.38 & 8.36 & \\
\hline 7 & 8.32 & 8.23 & 7.75 & 7.89 & 7.80 & 8.15 & 8.21 & 8.38 & 8.38 & 8.40 & 8.36 & \\
\hline 8 & 8.33 & 8.24 & 7.76 & 7.89 & 7.79 & 8.14 & 8.21 & 8.35 & 8.41 & 8.41 & 8.36 & \\
\hline 9 & 8.34 & 8.24 & 7.80 & 7.88 & 7.79 & 8.12 & 8.21 & 8.39 & 8.43 & 8.44 & 8.37 & \\
\hline 10 & 8.34 & 8.25 & 7.93 & 7.88 & 7.78 & 8.11 & 8.22 & 8.40 & 8.46 & 8.46 & 8.37 & 8.2 \\
\hline 11 & 8.34 & 8.22 & 7.96 & 7.87 & 7.77 & 8.11 & 8.23 & 8.52 & 8.51 & 8.47 & 8.36 & 8.2 \\
\hline 12 & 8.36 & 8.18 & 7.97 & 7.87 & 7.77 & 8.11 & 8.23 & 8.54 & 8.50 & 8.55 & 8.37 & 8.2 \\
\hline 13 & 8.35 & 8.15 & 8.00 & 7.86 & 7.76 & 8.10 & 8.22 & 8.56 & 8.49 & 8.51 & 8.36 & 8.2 \\
\hline 14 & 8.39 & 8.15 & 8.02 & 7.86 & 7.76 & 8.10 & 8.21 & 8.52 & 8.48 & 8.47 & 8.35 & 8.2 \\
\hline 15 & 8.36 & 8.16 & 8.01 & 7.85 & 7.75 & 8.11 & 8.21 & 8.48 & 8.49 & 8.43 & 8.35 & 8.2 \\
\hline 16 & 8.33 & 8.16 & 7.90 & 7.85 & 7.74 & 8.10 & 8.21 & 8.41 & 8.48 & 8.42 & 8.34 & \\
\hline 17 & 8.30 & 8.16 & 7.90 & 7.87 & 7.74 & 8.09 & 8.29 & 8.34 & 8.55 & 8.44 & 8.36 & \\
\hline 18 & 8.27 & 8.22 & 7.90 & 7.86 & 7.73 & 8.09 & 8.31 & 8.42 & 8.49 & 8.42 & 8.36 & 8. \\
\hline 19 & 8.26 & 8.24 & 7.90 & 7.85 & 7.73 & 8.08 & 8.34 & 8.40 & 8.46 & 8.40 & 8.38 & \\
\hline 20 & 8.23 & 8.25 & 7.88 & 7.84 & 7.74 & 8.07 & 8.40 & 8.34 & 8.42 & 8.38 & 8.38 & 8. \\
\hline 21 & 8.24 & 8.27 & 7.88 & 7.83 & 7.76 & 8.07 & 8.51 & 8.42 & 8.45 & 8.38 & 8.39 & \\
\hline 22 & 8.25 & 8.28 & 7.89 & 7.82 & 7.76 & 8.05 & 8.45 & 8.61 & 8.48 & 8.40 & 8.38 & \\
\hline 23 & 8.25 & 8.25 & 7.89 & 7.82 & 7.77 & 8.05 & 8.36 & 8.67 & 8.46 & 8.39 & 8.39 & \\
\hline 24 & 8.27 & 8.15 & 7.90 & 7.83 & 7.78 & 8.07 & 8.40 & 8.69 & 8.47 & 8.37 & 8.36 & 8.1 \\
\hline 25 & 8.33 & 8.08 & 7.89 & 7.82 & 7.78 & 8.10 & 8.43 & 8.69 & 8.46 & 8.35 & 8.37 & \\
\hline 26 & 8.33 & 8.01 & 7.89 & 7.83 & 7.78 & 8.22 & 8.43 & 8.67 & 8.46 & 8.34 & 8.38 & 8.2 \\
\hline 27 & 8.33 & 7.94 & 7.89 & 7.83 & 7.79 & 8.23 & 8.39 & 8.63 & 8.45 & 8.34 & 8.46 & \\
\hline 28 & 8.33 & 7.85 & 7.91 & 7.83 & 7.80 & 8.24 & 8.33 & 8.57 & 8.45 & 8.33 & 8.48 & \\
\hline 29 & 8.33 & 7.76 & 7.92 & 7.82 & 7.81 & 8.28 & 8.33 & 8.49 & 8.43 & 8.33 & 8.43 & \\
\hline 30 & 8.35 & 7.68 & 7.92 & 7.81 & --- & 8.30 & 8.37 & 8.45 & 8.42 & 8.34 & 8.37 & \\
\hline 31 & 8.35 & -- & 7.91 & 7.80 & $\ldots$ & 8.28 & -. & 8.42 & -- & 8.34 & 8.37 & \\
\hline MEAN & 8.31 & 8.20 & 7.86 & 7.86 & 7.77 & 8.11 & 8.29 & 8.47 & 8.46 & 8.40 & 8.38 & \\
\hline MAX & 8.39 & 8.61 & 8.02 & 7.93 & 7.81 & 8.30 & 8.51 & 8.69 & 8.55 & 8.55 & 8.56 & \\
\hline MIN & 8.23 & 7.68 & 7.64 & 7.80 & 7.73 & 7.84 & 8.18 & 8.34 & 8.38 & 8.33 & 8.34 & \\
\hline
\end{tabular}




\section{NAGAWICKA LAKE, AT DEEP HOLE, AT DELAFIELD, WI}

LOCATION.--Lat 4304'17", long 88 23'03", in SE 1/4 NE 1/4 sec.17, T.7 N., R.18 E., Waukesha County, Hydrologic Unit 07090001, at Delafield. PERIOD OF RECORD.--February 2003 to current year.

REMARKS.--Lake sampled near center at the deep hole. Lake ice-covered during February sampling. Water-quality analyses done by Wisconsin State Laboratory of Hygiene.

WATER-QUALITY DATA, FEBRUARY 19 TO JUNE 7, 2004

(Milligrams per liter unless otherwise indicated)

Date

Lake stage (ft)

Secchi depth (m)

Depth of sample $(m)$

Chlorophyll a, phytoplankton ( $\mu \mathrm{g} / \mathrm{L}$ )

Water temperature $\left({ }^{\circ} \mathrm{C}\right)$

Specific conductance $(\mu \mathrm{S} / \mathrm{cm})$

$\mathrm{pH}$

Dissolved oxygen (mg/L)

Phosphorus, total (as $\mathrm{P}$ )

Phosphorus, ortho, dissolved (as $\mathrm{P}$ )

Nitrogen, $\mathrm{NO}_{2}+\mathrm{NO}_{3}$, diss. (as $\mathrm{N}$ )

Nitrogen, ammonia, dissolved (as $\mathrm{N}$ )

Nitrogen, amm. + org., total (as N)

Nitrogen, total (as $\mathrm{N}$ )

Color (Pt-Co. scale)

Turbidity (NTU)

Hardness, as $\mathrm{CaCO}_{3}$

Calcium, dissolved (Ca)

Magnesium, dissolved $(\mathrm{Mg})$

Sodium, dissolved $(\mathrm{Na})$

Potassium, dissolved (K)

Chloride, dissolved $(\mathrm{Cl})$

Solids, dissolved, at $180^{\circ} \mathrm{C}$

Iron, dissolved $(\mathrm{Fe})(\mu \mathrm{g} / \mathrm{L})$

Manganese, dissolved, $(\mathrm{Mn})(\mu \mathrm{g} / \mathrm{L})$

\begin{tabular}{ccccc} 
& $\frac{\text { Feb-19 }}{7.73}$ & & \multicolumn{2}{c}{$\frac{\text { Apr-14 }}{8.21}$} \\
0.5 & - & & 0.5 & \\
- & & 26.5 & 1.2 & \\
0.3 & & - & 7.1 & \\
804 & 1.8 & 731 & \\
8.0 & 877 & 12.3 & \\
14.2 & 7.5 & 0.012 \\
0.008 & 8.1 & $<0.002$ \\
- & 0.016 & 0.942 \\
- & - & 0.098 \\
- & - & 0.58 \\
- & - & 1.5 \\
- & - & 5 \\
- & - & $<1.0$ \\
- & - & 300 \\
- & - & 56.7 \\
- & - & 38.3 \\
- & - & 33.4 \\
- & - & 2 \\
-- & - & 74.1 \\
- & - & 414 \\
- & - & $<100$ \\
- & - & $<1$ \\
- & - &
\end{tabular}

26

6.0

733

8.1

11.3

0.017

$-$

$-$

$-$

$-$

$-$

$-$

$-$

$-$

$-$

$-$
2-19-04

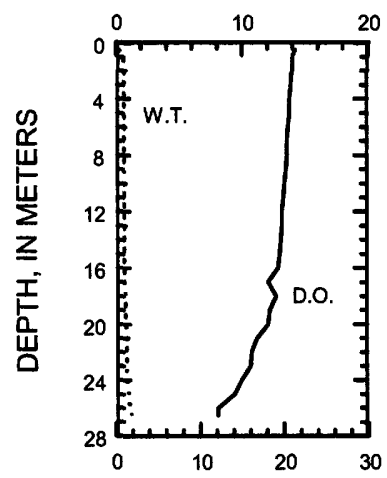

4-14-04

DISSOLVED OXYGEN (D.O.) IN MILLIGRAMS PER LITER

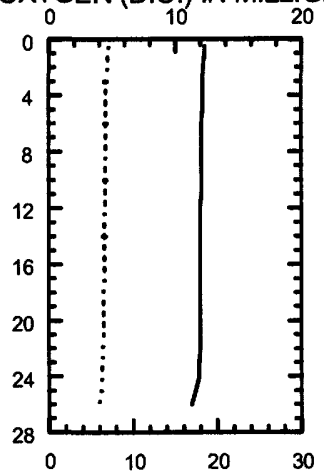

WATER TEMPERATURE (W.T.) IN DEGREES CELSIUS

PHIN STANDARD UNITS

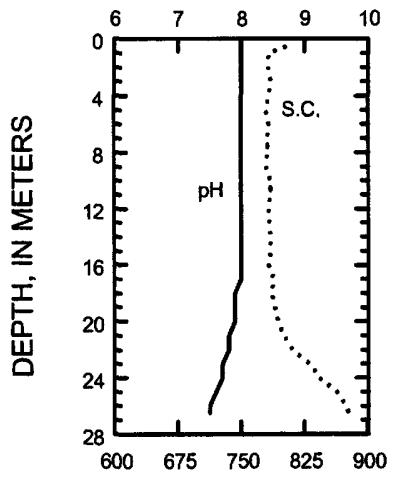

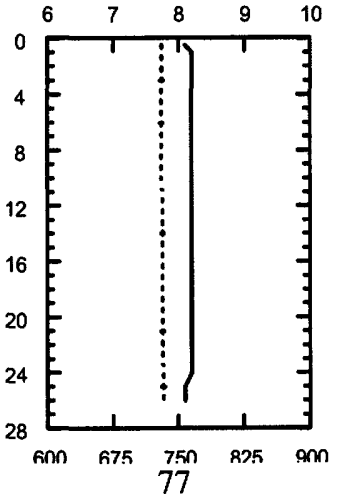

6-7-04

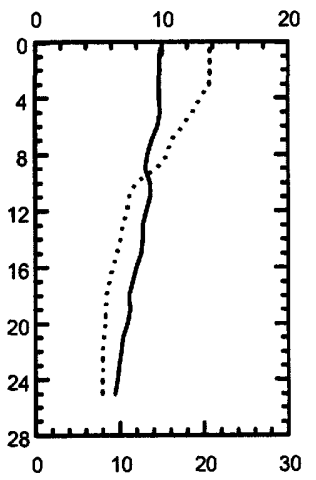


WATER-QUALITY DATA, JULY 14 TO SEPTEMBER 23, 2004

(Milligrams per liter unless otherwise indicated)
Date

Lake stage ( $\mathrm{ft}$ )

Secchi depth $(\mathrm{m})$

Depth of sample $(m)$

Chlorophyll a, phytoplankton ( $\mu \mathrm{g} / \mathrm{L})$

Water temperature $\left({ }^{\circ} \mathrm{C}\right)$

Specific conductance $(\mu \mathrm{S} / \mathrm{cm})$

$\mathrm{pH}$

Dissolved oxygen (mg/L)

Phosphorus, total (as P)

Phosphorus, ortho, dissolved (as P)

Nitrogen, $\mathrm{NO}_{2}+\mathrm{NO}_{3}$, diss. (as $\mathrm{N}$ )

Nitrogen, ammonia, dissolved (as $\mathrm{N}$ )

Nitrogen, amm. + diss., total (as N)

$7-14-04$
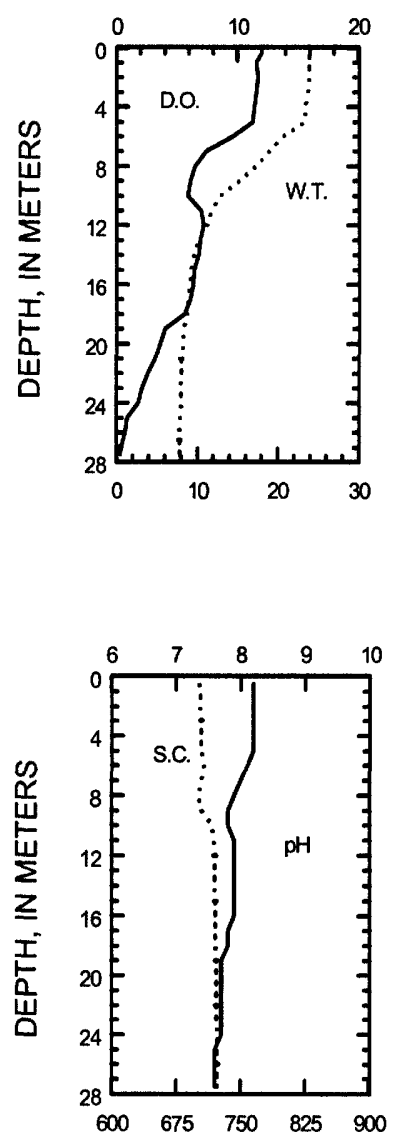

8-17-04

DISSOLVED OXYGEN (D.O.) IN MILIGRAMS PER LITER

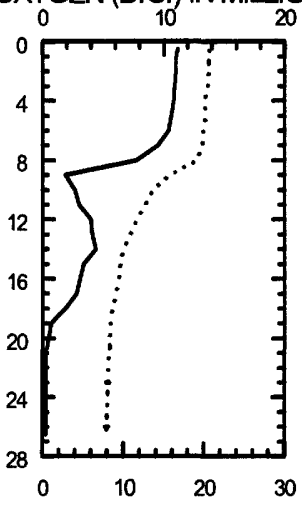

WATER TEMPERATURE (W.T.) IN DEGREES CELSIUS

PHIN STANDARD UNITS

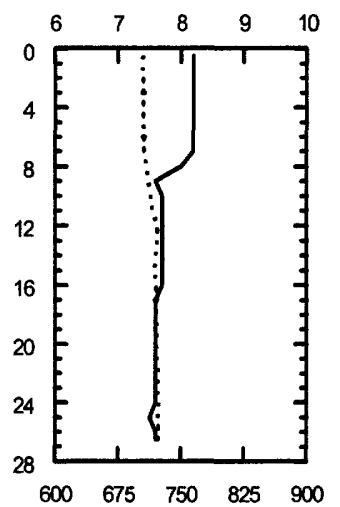

9-23-04
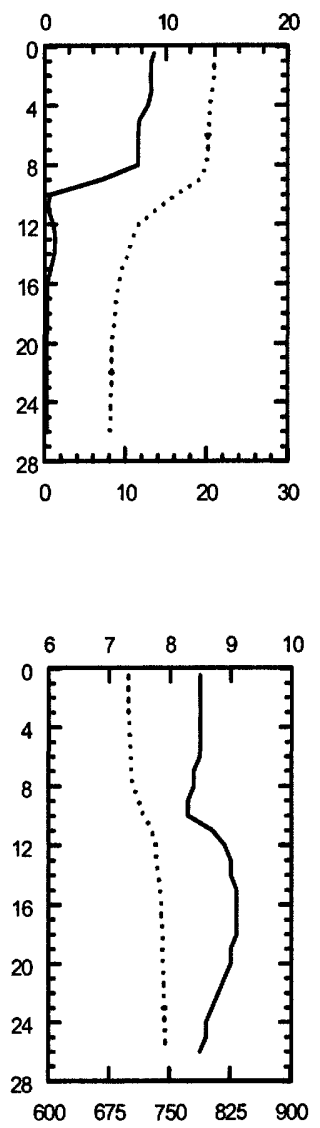

SPECIFIC CONDUCTANCE (S.C.) IN MICROSIEMENS PER CENTIMETER AT 25 DEGREES CELSIUS 

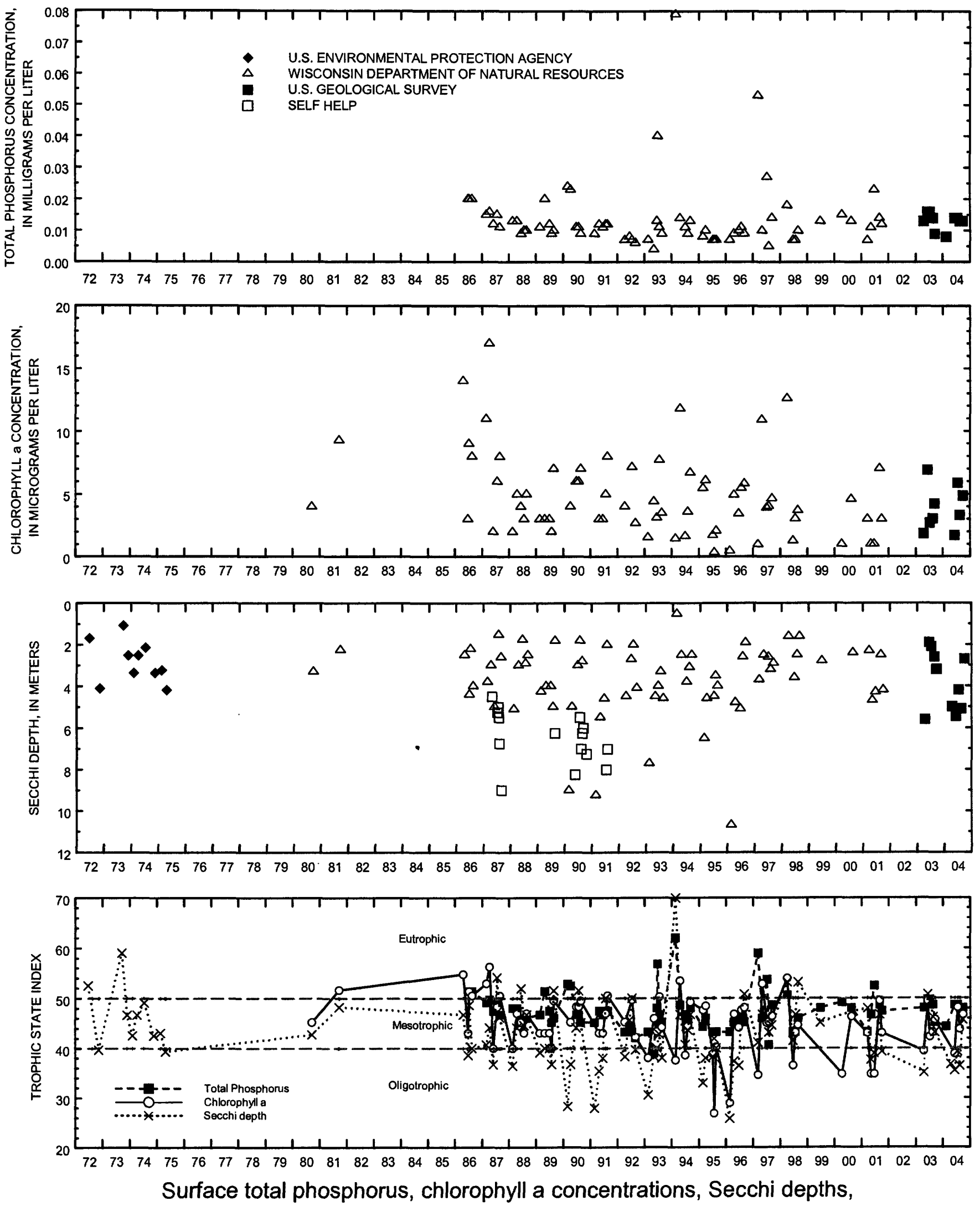

and TSI data for Nagawicka Lake at Delafield, Wisconsin. 


\section{OCONOMOWOC LAKE NO. 1 (CENTER) AT OCONOMOWOC, WI}

LOCATION.--Lat $43^{\circ} 05^{\prime} 51^{\prime \prime}$, long $88^{\circ} 27^{\prime} 35^{\prime \prime}$, in NW 1/4 SE 1/4 sec.2, T.7 N., R.17 E., Waukesha County, Hydrologic Unit 07090001, at Oconomowoc.

PERIOD OF RECORD.--March 1986 to current year.

REMARKS.--Lake sampled near center at the deep hole. Lake ice-covered during February sampling. Water-quality analyses done by Wisconsin State Laboratory of Hygiene.

WATER-QUALITY DATA, FEBRUARY 19 TO AUGUST 17, 2004

Date

Lake stage (ft)

Secchi depth (m)

Depth of sample (m)

Chlorophyll a, phytoplankton ( $\mu \mathrm{g} / \mathrm{L}$ )

Water temperature $\left({ }^{\circ} \mathrm{C}\right)$

Specific conductance $(\mu \mathrm{S} / \mathrm{cm})$

$\mathrm{pH}$

Dissolved oxygen (mg/L)

Phosphorus, total (as P)

Phosphorus, ortho, dissolved (as $\mathrm{P}$ )

Nitrogen, $\mathrm{NO}_{2}+\mathrm{NO}_{3}$, diss. (as $\mathrm{N}$ )

Nitrogen, ammonia, dissolved (as $\mathrm{N}$ )

Nitrogen, amm. + org., total (as N)

Nitrogen, total (as $\mathrm{N}$ )

Color (Pt-Co. scale)

Turbidity (NTU)

Hardness, as $\mathrm{CaCO}_{3}$

Calcium, dissolved ( $\mathrm{Ca}$ )

Magnesium, dissolved (Mg)

Sodium, dissolved $(\mathrm{Na})$

Potassium, dissolved (K)

Alkalinity, as $\mathrm{CaCO}_{3}$

Sulfate, dissolved $\left(\mathrm{SO}_{4}\right)$

Chloride, dissolved $(\mathrm{Cl})$

Silica, dissolved $\left(\mathrm{SiO}_{2}\right)$

Solids, dissolved, at $180^{\circ} \mathrm{C}$

Iron, dissolved $(\mathrm{Fe})(\mu \mathrm{g} / \mathrm{L})$

Manganese, dissolved, $(\mathrm{Mn})(\mu \mathrm{g} / \mathrm{L})$

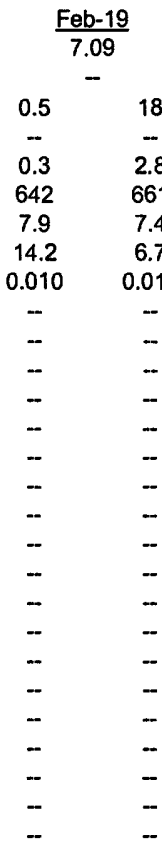

2-19-04

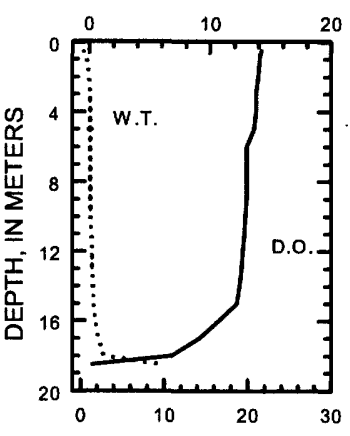

DISSOLVED OXYGEN (D.O.) IN MILLIGRAMS PER LITER
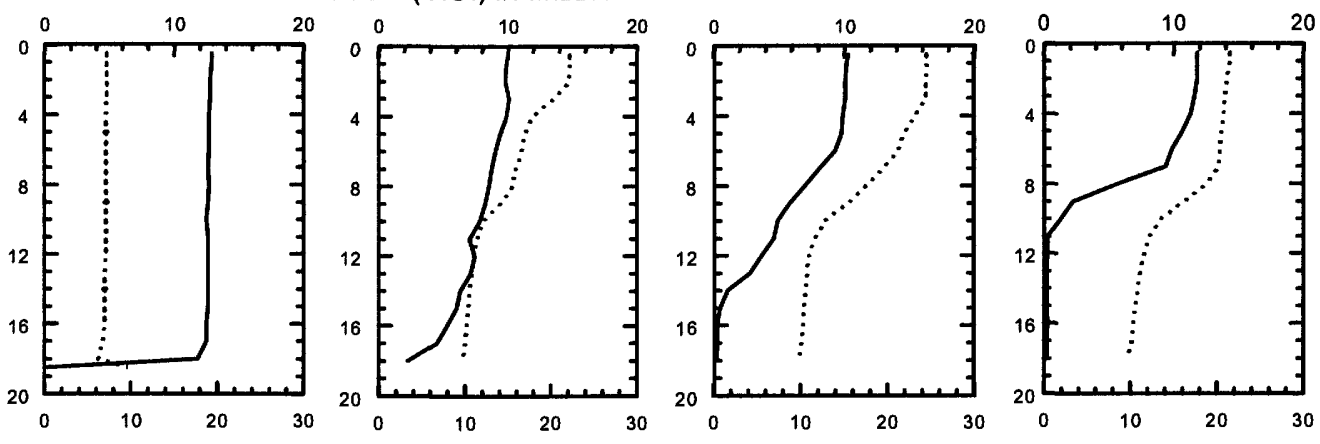

WATER TEMPERATURE (W.T.) IN DEGREES CELSIUS

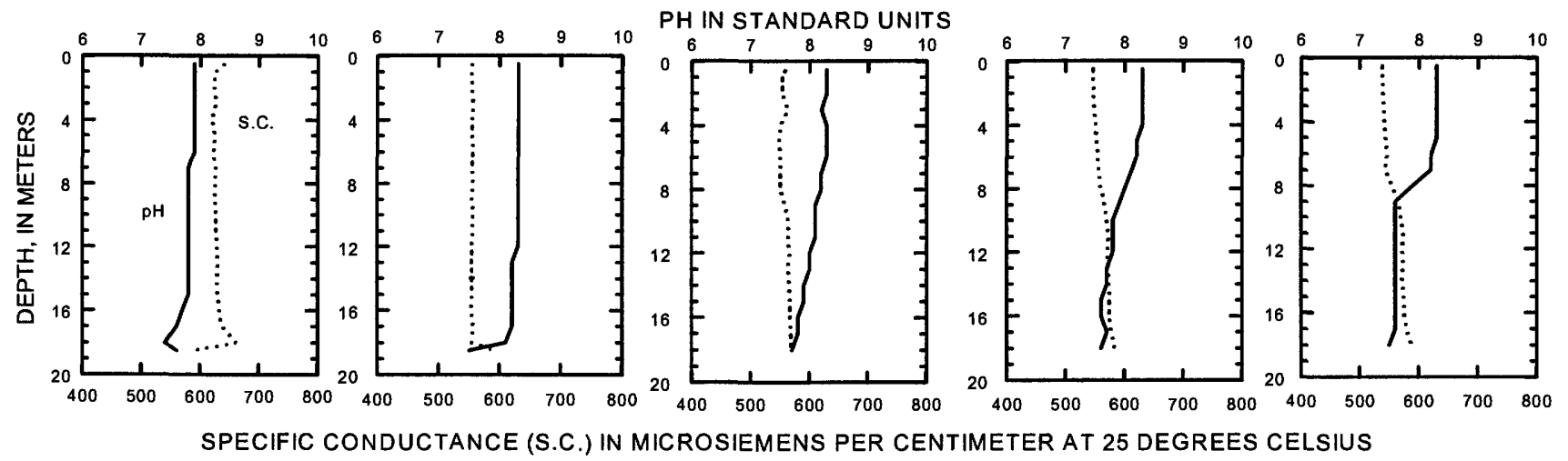



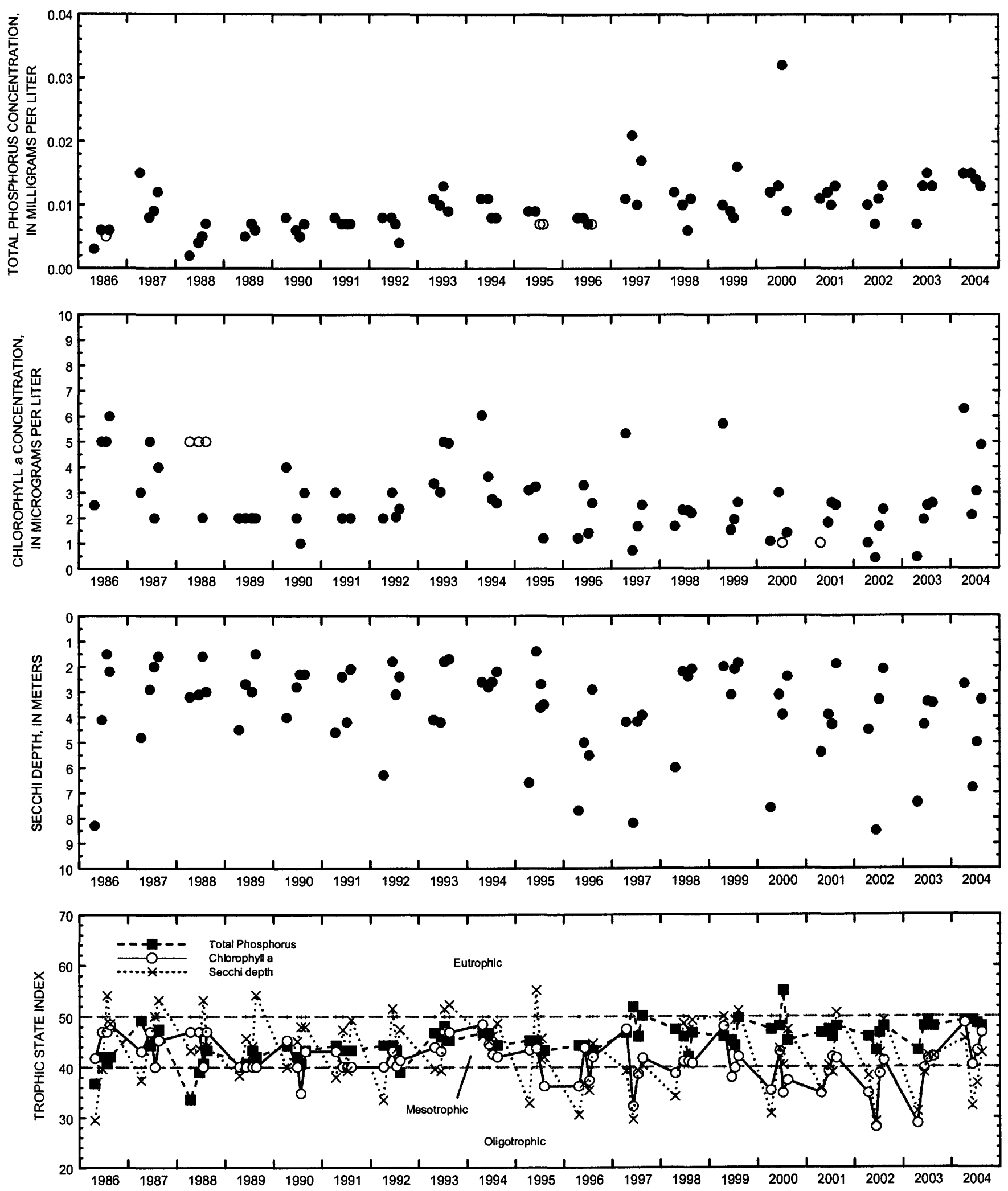

Surface total phosphorus, chlorophyll a concentrations, Secchi depths, and TSI data for Oconomowoc Lake, Center Site, at Oconomowoc, Wisconsin. 


\section{OCONOMOWOC LAKE NO. 2 (OFF HEWITT POINT) AT OCONOMOWOC, WI}

LOCATION.--Lat $43^{\circ} 06^{\prime} 09^{\prime \prime}$, long $88^{\circ} 26^{\prime} 22^{\prime \prime}$, in NW 1/4 NW 1/4 sec.1, T.7 N., R.17 E., Waukesha County, Hydrologic Unit 07090001, at Oconomowoc.

PERIOD OF RECORD.--March 1986 to current year.

REMARKS.--Lake sampled at the deepest point in northeast bay near Hewitt Point. Lake ice-covered during February sampling. Water-quality analyses done by Wisconsin State Laboratory of Hygiene.

WATER-QUALITY DATA, FEBRUARY 19 TO AUGUST 17, 2004

(Milligrams per liter unless otherwise indicated)

Date

Lake stage (ft)

Secchi depth (m)

Depth of sample (m)

Chlorophyll a, phytoplankton $(\mu \mathrm{g} / \mathrm{L})$

Water temperature $\left({ }^{\circ} \mathrm{C}\right)$

Specific conductance $(\mu \mathrm{S} / \mathrm{cm})$

$\mathrm{pH}$

Dissolved oxygen ( $\mathrm{mg} / \mathrm{L}$ )

Phosphorus, total (as P)

\begin{tabular}{|c|c|c|c|c|c|c|c|c|c|}
\hline \multicolumn{2}{|c|}{ Feb-19 } & \multicolumn{2}{|c|}{ Apr-13 } & \multicolumn{2}{|c|}{ Jun-8 } & \multicolumn{2}{|c|}{ Jul-15 } & \multicolumn{2}{|c|}{ Aug-17 } \\
\hline \multicolumn{2}{|c|}{7.09} & \multicolumn{2}{|c|}{7.82} & \multicolumn{2}{|c|}{8.23} & \multicolumn{2}{|c|}{7.11} & \multicolumn{2}{|c|}{7.03} \\
\hline \multicolumn{2}{|c|}{-} & \multicolumn{2}{|c|}{6.2} & \multicolumn{2}{|c|}{7.6} & \multicolumn{2}{|c|}{4.8} & \multicolumn{2}{|c|}{3.1} \\
\hline 0.5 & 14 & 0.5 & 14.5 & 0.5 & 14.5 & 0.5 & 14.5 & 0.5 & 14 \\
\hline- & - & 1.1 & - & 1.3 & - & 2.1 & - & 4.9 & - \\
\hline 1.0 & 4.2 & 7.9 & 7.6 & 22.2 & 11.4 & 24.6 & 11.7 & 21.9 & 11.6 \\
\hline 660 & 711 & 602 & 600 & 557 & 614 & 585 & 628 & 580 & 644 \\
\hline 7.8 & 7.3 & 8.2 & 8.1 & 8.2 & 7.6 & 8.1 & 7.5 & 8.3 & 7.5 \\
\hline 13.3 & 6.1 & 12.2 & 11.9 & 9.9 & 1.2 & 9.9 & 0.3 & 11.9 & 0.3 \\
\hline 0.008 & 0.009 & 0.012 & 0.013 & 0.007 & 0.022 & 0.015 & 0.026 & 0.010 & 0.032 \\
\hline
\end{tabular}

2-19-04

4-13-04

6-8-04

7-15-04

8-17-04

DISSOLVED OXYGEN (D.O.) IN MILLIGRAMS PER LITER
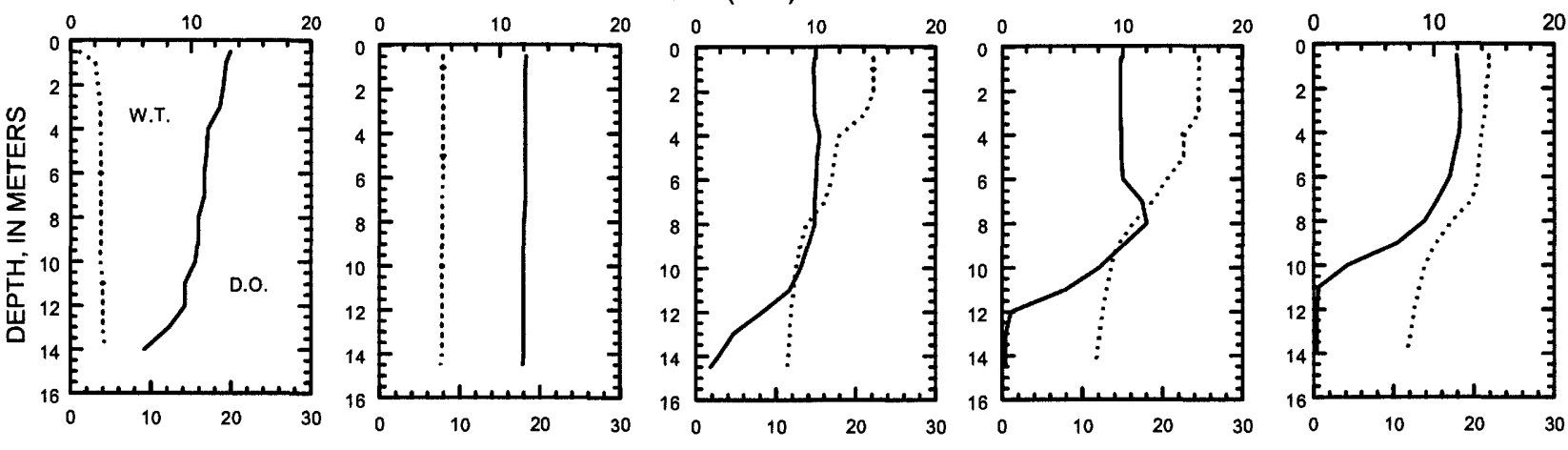

WATER TEMPERATURE (W.T.) IN DEGREES CELSIUS

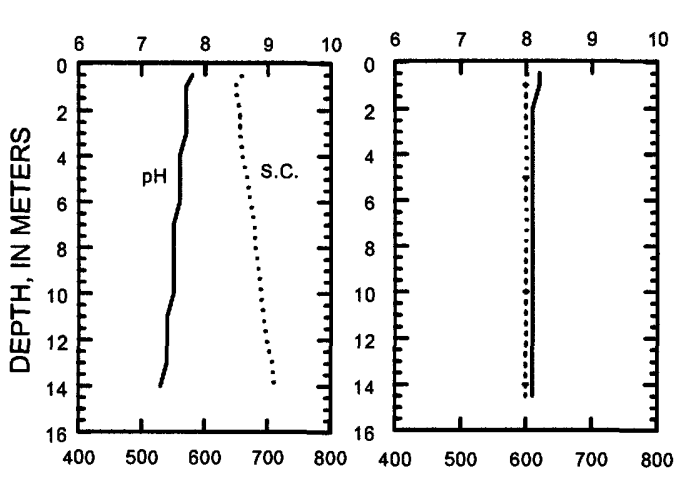

PH IN STANDARD UNITS
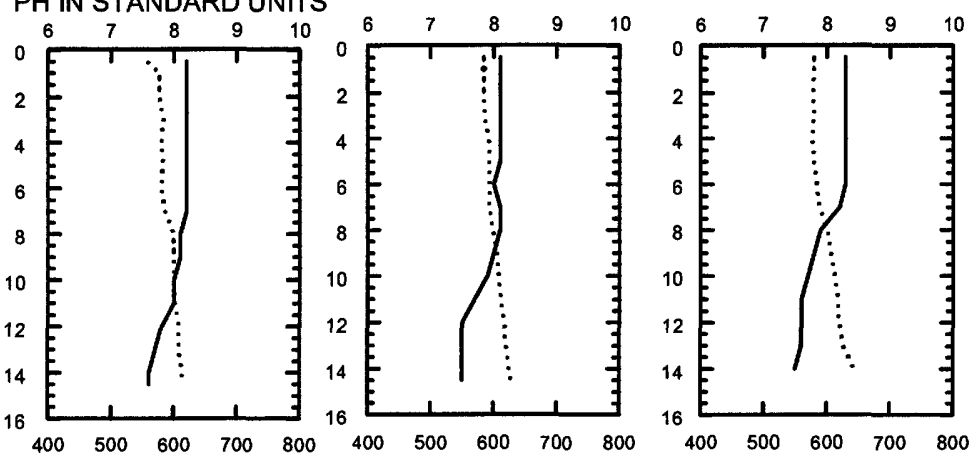

SPECIFIC CONDUCTANCE (S.C.) IN MICROSIEMENS PER CENTIMETER AT 25 DEGREES CELSIUS 

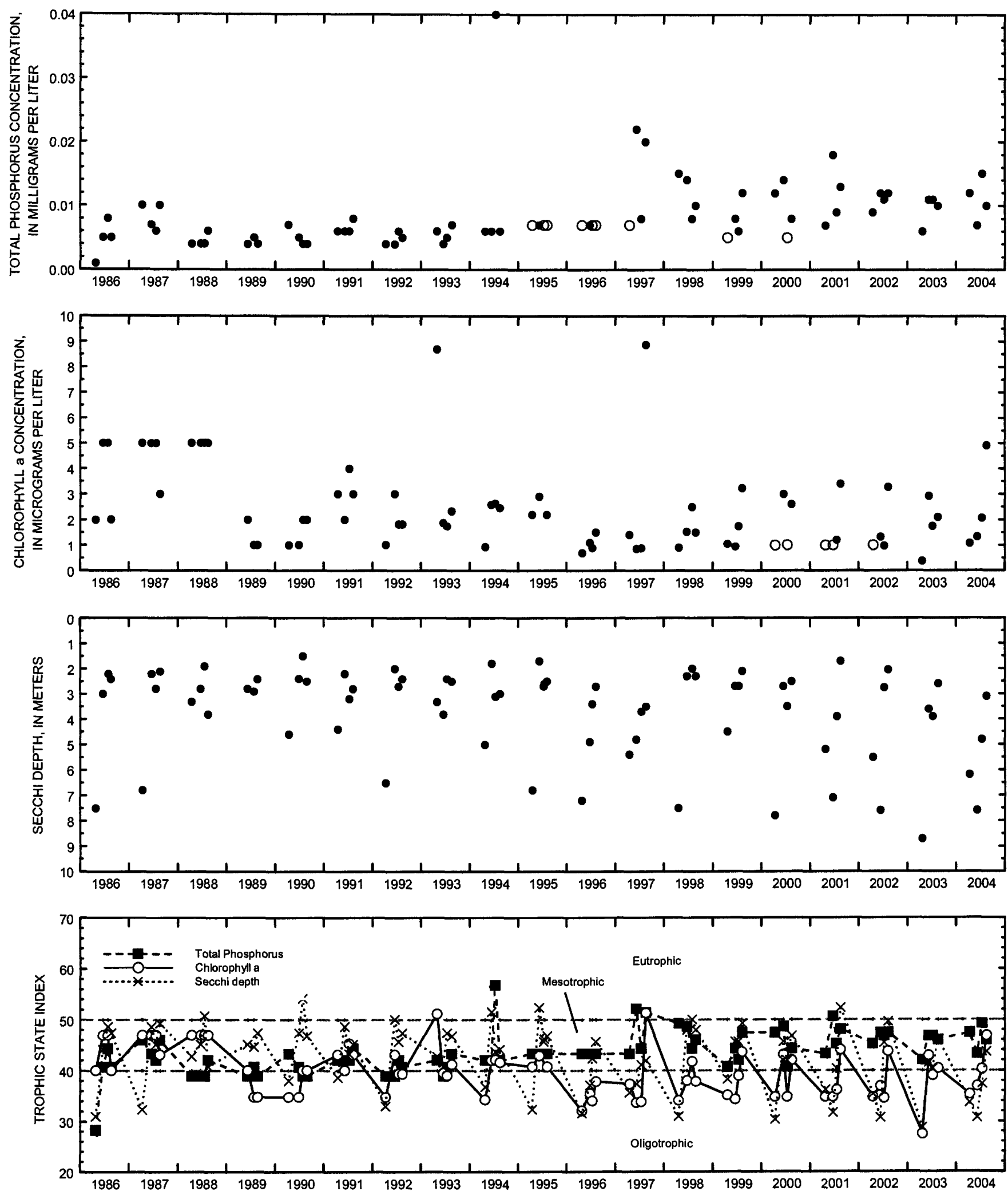

Surface total phosphorus, chlorophyll a concentrations, Secchi depths, and TSI data for Oconomowoc Lake, Hewitt Point, at Oconomowoc, Wisconsin.

(Circles on the first two plots indicate laboretory detection limit for selected analyses. Actual concentrations for these particular analyses are less than the plotted circles.) 


\section{OKAUCHEE LAKE AT OKAUCHEE, WI}

LOCATION.--Lat $43^{\circ} 07^{\prime} 23^{\prime \prime}$, long $88^{\circ} 25^{\prime} 21^{\prime \prime}$, in SE 1/4 SE 1/4, sec.25, T.8 N., R.17 E., Waukesha County, Hydrologic Unit 07090001, at Okauchee. DRAINAGE AREA.--80.7 $\mathrm{mi}^{2}$.

PERIOD OF RECORD.--February 1984 to current year.

LAKE-STAGE GAGE.--Datum of gage is $869.00 \mathrm{ft}$ above sea level.

REMARKS.--Lake sampled near center at the deep hole. Lake ice-covered during February sampling. Water-quality analyses done by Wisconsin State Laboratory of Hygiene.

$$
\begin{aligned}
& \text { WATER-QUALITY DATA, FEBRUARY } 19 \text { TO AUGUST 17, } 2004 \\
& \text { (Milligrams per liter unless otherwise indicated) }
\end{aligned}
$$

Date

Lake stage (ft)

Secchi depth (m)

Depth of sample (m)

Chlorophyll a, phytoplankton ( $\mu \mathrm{g} / \mathrm{L}$ )

Water temperature $\left({ }^{\circ} \mathrm{C}\right)$

Specific conductance $(\mu \mathrm{S} / \mathrm{cm})$

$\mathrm{pH}$

Dissolved oxygen (mg/L)

Phosphorus, total (as P)

Phosphorus, ortho, dissolved (as P)

Nitrogen, $\mathrm{NO}_{2}+\mathrm{NO}_{3}$, diss. (as $\mathrm{N}$ )

Nitrogen, ammonia, dissolved (as $N$ )

Nitrogen, amm. + diss., total (as N)

Nitrogen, amm. + org., total (as $\mathrm{N}$ )

Nitrogen, total (as N)

Color (Pt-Co. scale)

Turbidity (NTU)

Hardness, as $\mathrm{CaCO}_{3}$

Calcium, dissolved (Ca)

Magnesium, dissolved (Mg)

Sodium, dissolved ( $\mathrm{Na}$ )

Potassium, dissolved (K)

Alkalinity, as $\mathrm{CaCO}_{3}$

Sulfate, dissolved $\left(\mathrm{SO}_{4}\right)$

Chloride, dissolved $(\mathrm{Cl})$

Silica, dissolved $\left(\mathrm{SiO}_{2}\right)$

Solids, dissolved, at $180^{\circ} \mathrm{C}$

Iron, dissolved (Fe) $(\mu \mathrm{g} / \mathrm{L})$

\begin{tabular}{|c|c|c|c|}
\hline \multicolumn{2}{|c|}{ Feb-19 } & \multicolumn{2}{|c|}{ Apr-13 } \\
\hline \multicolumn{2}{|c|}{3.88} & \multicolumn{2}{|c|}{4.72} \\
\hline \multicolumn{2}{|c|}{-} & \multicolumn{2}{|c|}{1.7} \\
\hline 0.5 & 27.5 & 0.5 & 25.5 \\
\hline- & - & 11.9 & - \\
\hline 0.4 & 2.2 & 6.6 & 5.6 \\
\hline 637 & 644 & 555 & 552 \\
\hline 8.1 & 7.7 & 8.3 & 8.1 \\
\hline 13.7 & 4.1 & 13.5 & 12.3 \\
\hline 0.014 & 0.014 & 0.027 & 0.020 \\
\hline- & -- & $<0.002$ & -- \\
\hline-- & - & 0.261 & -- \\
\hline- & - & 0.002 & -- \\
\hline - & - & - & -- \\
\hline - & -. & 0.6 & - \\
\hline- & - & 0.86 & -- \\
\hline - & -- & 10 & -- \\
\hline- & -- & 2.7 & -- \\
\hline- & -- & 260 & -- \\
\hline - & - & 47.9 & -- \\
\hline -- & - & 34.2 & -- \\
\hline - & - & 15.5 & -- \\
\hline- & - & 2 & -- \\
\hline -- & -- & 216 & - \\
\hline -- & - & 26.9 & - \\
\hline - & - & 37.5 & -- \\
\hline-- & - & 2.74 & -- \\
\hline -. & -- & 318 & - \\
\hline-- & - & $<100$ & -. \\
\hline-- & - & $<1$ & - \\
\hline
\end{tabular}

Manganese, dissolved, $(\mathrm{Mn})(\mu \mathrm{g} / \mathrm{L})$
4-13-04

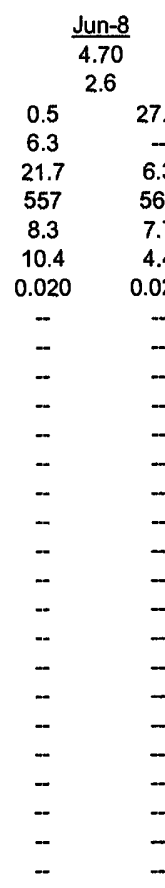

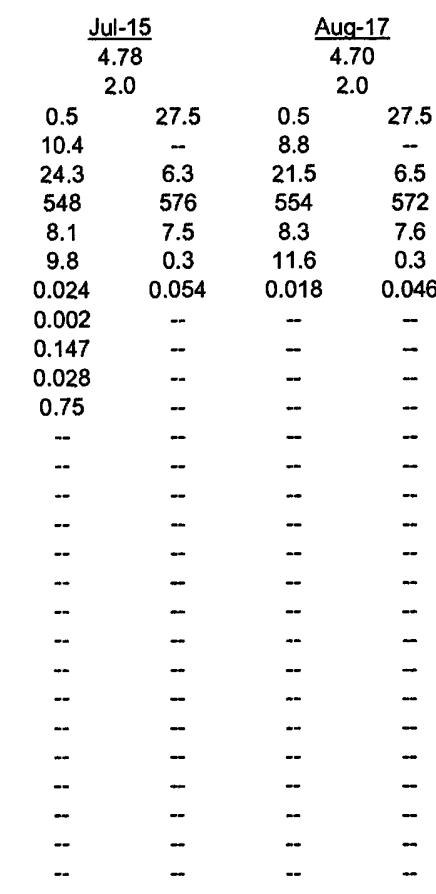

7-15-04

DISSOLVED OXYGEN (D.O.) IN MILLIGRAMS PER LITER
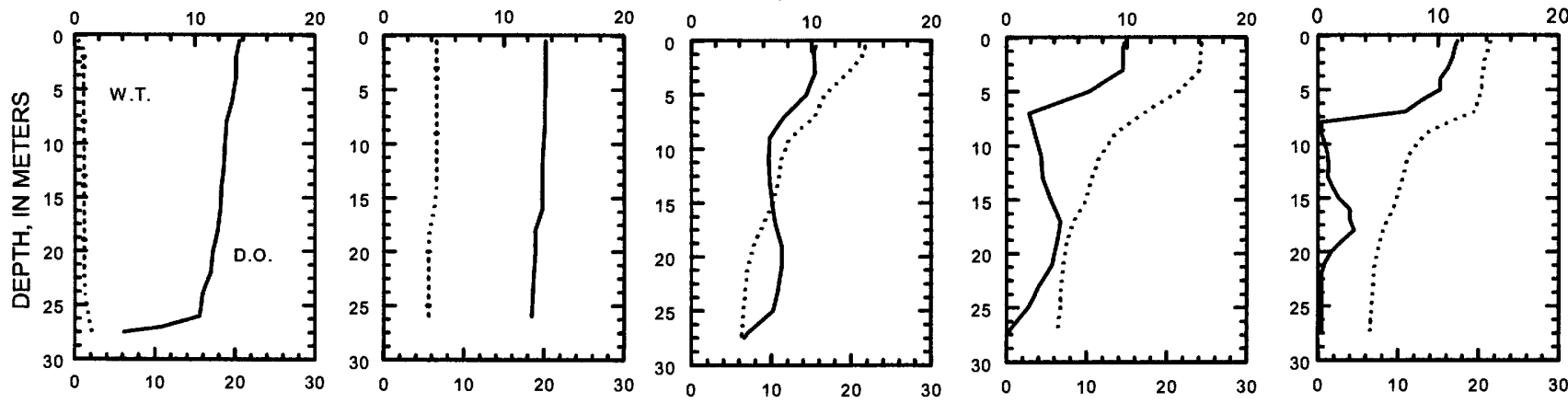

WATER TEMPERATURE (W.T.) IN DEGREES CELSIUS

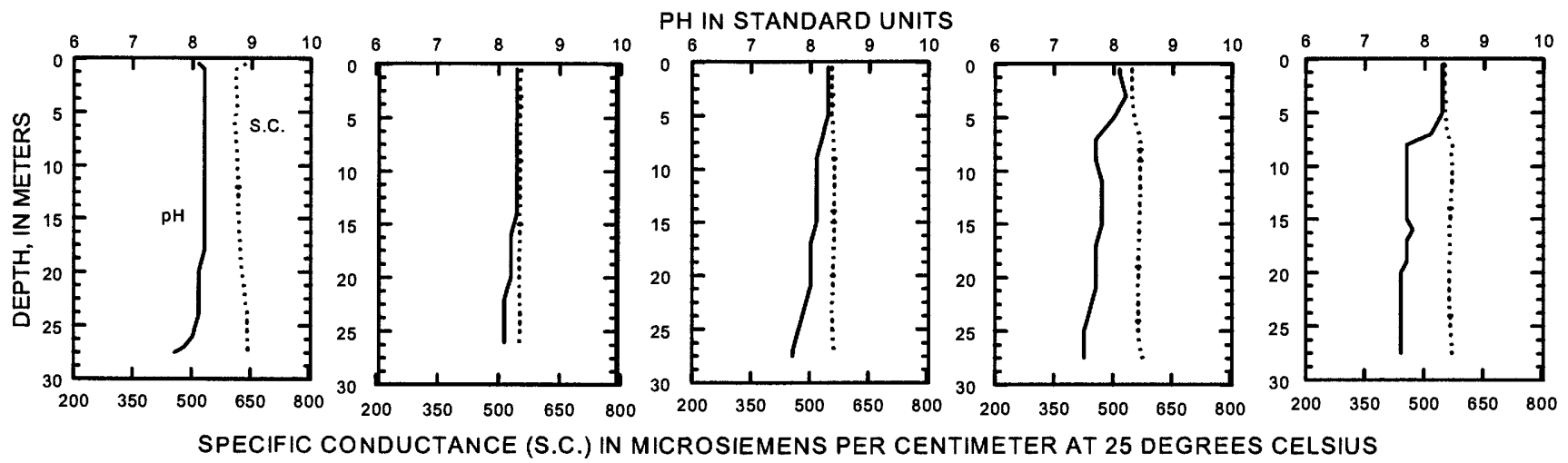



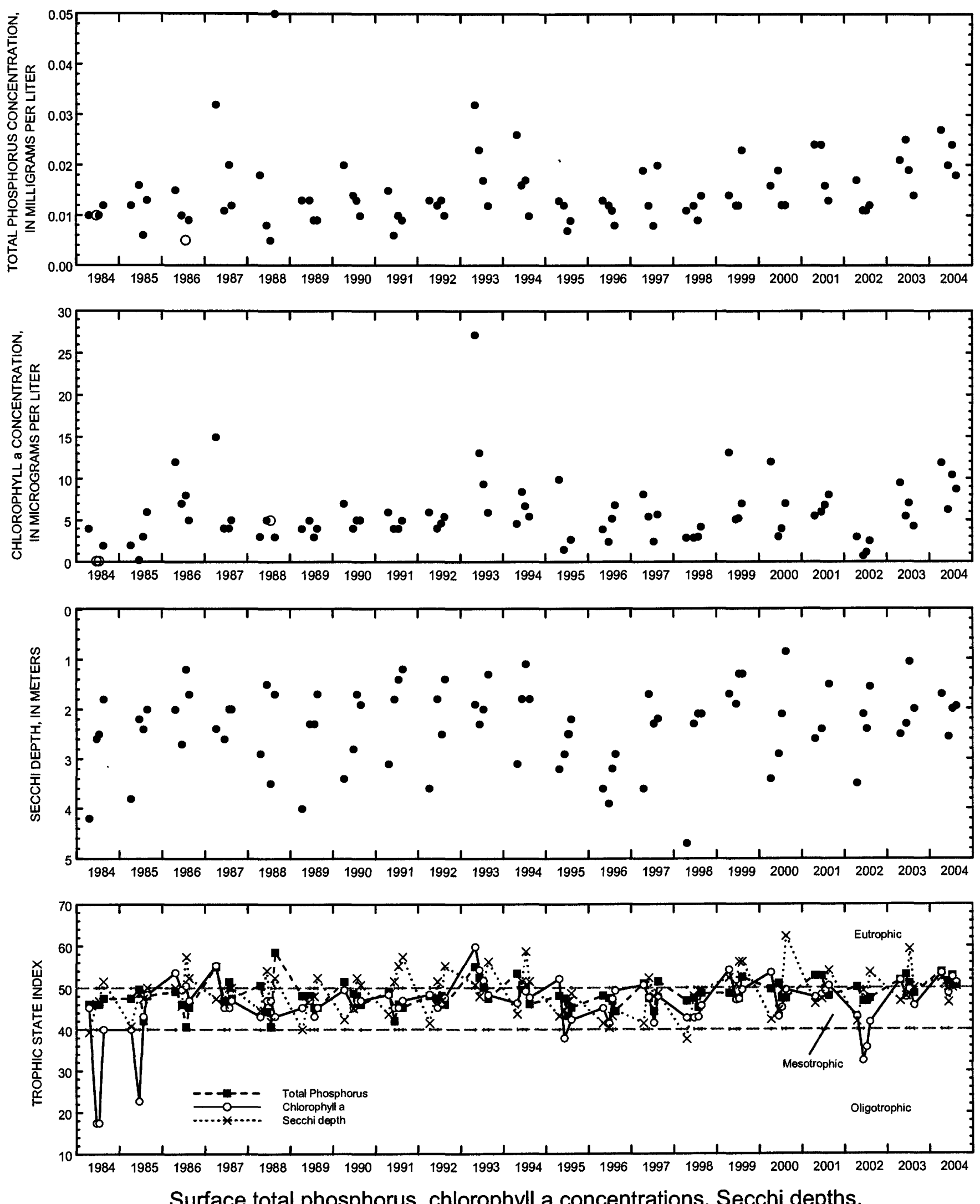

and TSI data for Okauchee Lake, near Okauchee, Wisconsin.

(Circles on the first two plots indicate laboratory detection limit for selected analyses. Actual concentrations for these particular analyses are less than the plotted circles.) 


\section{OKAUCHEE LAKE, NO. 1, NEAR OKAUCHEE, WI}

LOCATION.--Lat $43^{\circ} 07^{\prime} 59^{\prime \prime}$, long $88^{\circ} 24^{\prime} 42^{\prime \prime}$, in NE $1 / 4$ NW 1/4 sec.30, T.8 N., R.18 E., Waukesha County, Hydrologic Unit 07090001, near Okauchee.

PERIOD OF RECORD.--April 1986 to current year.

LAKE-STAGE GAGE.--Datum of gage is $869.00 \mathrm{ft}$ above sea level.

REMARKS.--Lake sampled in Crane's Nest Bay, in the northeast part of the lake, at an approximate depth of $2 \mathrm{~m}$. Water-quality analyses done by Wisconsin State Laboratory of Hygiene.

WATER-QUALITY DATA, APRIL 13 TO AUGUST 17, 2004

(Milligrams per liter unless otherwise indicated)

$\begin{array}{lcccc}\text { Date } & \text { Apr-13 } & \frac{\text { Jun-8 }}{\text { Jul-15 }} & \frac{\text { Aug-17 }}{4.70} \\ \text { Lake stage }(\mathrm{ft}) & 4.72 & 4.70 & 1.1 & 1.3 \\ \text { Secchi depth }(\mathrm{m}) & 1.8 & 0.5 & 0.5 & 0.5 \\ \text { Depth of sample }(\mathrm{m}) & 0.5 & 21.9 & 10.1 & 14.2 \\ \text { Chlorophyll a, phytoplankton }(\mu \mathrm{gg} / \mathrm{L}) & 9.2 & 24.7 & 25.2 & 21.7 \\ \text { Water temperature }\left({ }^{\circ} \mathrm{C}\right) & 6.1 & 575 & 592 & 565 \\ \text { Specific conductance }(\mu \mathrm{S} / \mathrm{cm}) & 552 & 8.4 & 7.9 & 8.2 \\ \text { pH } & 8.4 & 11.1 & 7.6 & 11.2 \\ \text { Dissolved oxygen }(\mathrm{mg} / \mathrm{L}) & 13.3 & 0.034 & 0.041 & 0.026\end{array}$



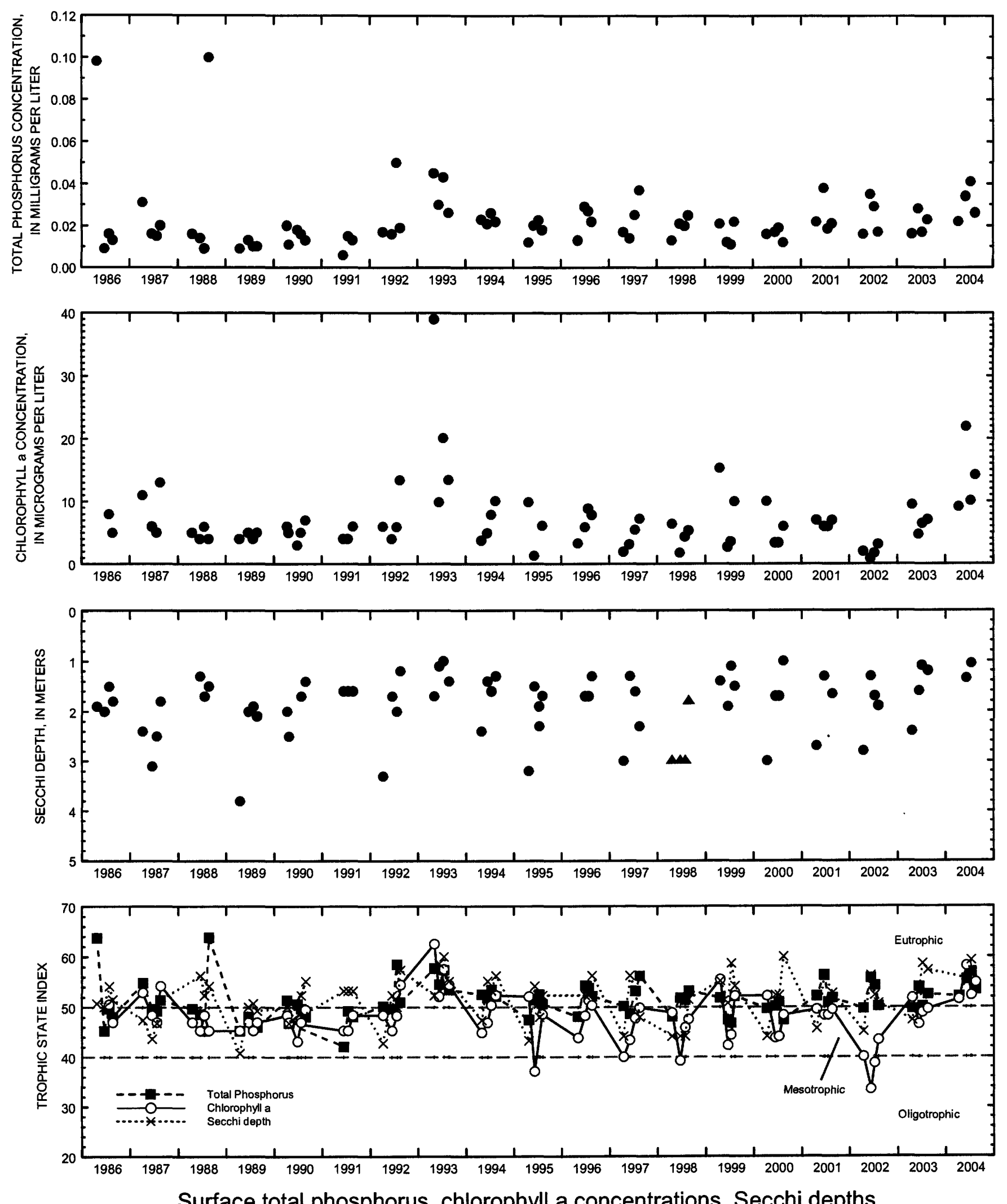

Surface total phosphorus, chlorophyll a concentrations, Secchi depths, and TSI data for Okauchee Lake, No. 1, near Okauchee, Wisconsin. 


\section{OKAUCHEE LAKE, NO. 2, AT OKAUCHEE, WI}

LOCATION.--Lat $43^{\circ} 06^{\prime} 45^{\prime \prime}$, long $88^{\circ} 26^{\prime} 45^{\prime \prime}$, in SE $1 / 4$ NE $1 / 4$ sec.35, T.8 N., R.17 E., Waukesha County, Hydrologic Unit 07090001 , at Okauchee.

PERIOD OF RECORD.--April 1986 to current year.

LAKE-STAGE GAGE.--Datum of gage is $869.00 \mathrm{ft}$ above sea level.

REMARKS.--Lake sampled in Lower Okauchee Lake, at an approximate depth of $5 \mathrm{~m}$. Water-quality analyses done by Wisconsin State Laboratory of Hygiene.

WATER-QUALITY DATA, APRIL 13 TO AUGUST 17, 2004

(Milligrams per liter unless otherwise indicated)

$\begin{array}{lcccc}\text { Date } & \text { Apr-13 } & \frac{\text { Jun-8 }}{\text { Jul- } 15} & & \frac{\text { Aug- } 17}{4.70} \\ \text { Lake stage }(\mathrm{ft}) & 4.72 & 4.70 & 4.78 & 1.7 \\ \text { Secchi depth }(\mathrm{m}) & 1.0 & 1.9 & 2.3 & 0.5 \\ \text { Depth of sample }(\mathrm{m}) & 0.5 & 0.5 & 3.7 & 14.7 \\ \text { Chlorophyll a, phytoplankton }(\mu \mathrm{g} / \mathrm{L}) & 10.1 & 23.9 & 25.5 & 21.8 \\ \text { Water temperature }\left({ }^{\circ} \mathrm{C}\right) & 8.2 & 549 & 534 & 533 \\ \text { Specific conductance }(\mu \mathrm{S} / \mathrm{cm}) & 544 & 8.3 & 8.1 & 8.3 \\ \mathrm{pH} & 8.5 & 11.2 & 10.0 & 13.4 \\ \text { Dissolved oxygen }(\mathrm{mg} / \mathrm{L}) & 13.1 & 0.019 & 0.020 & 0.026\end{array}$



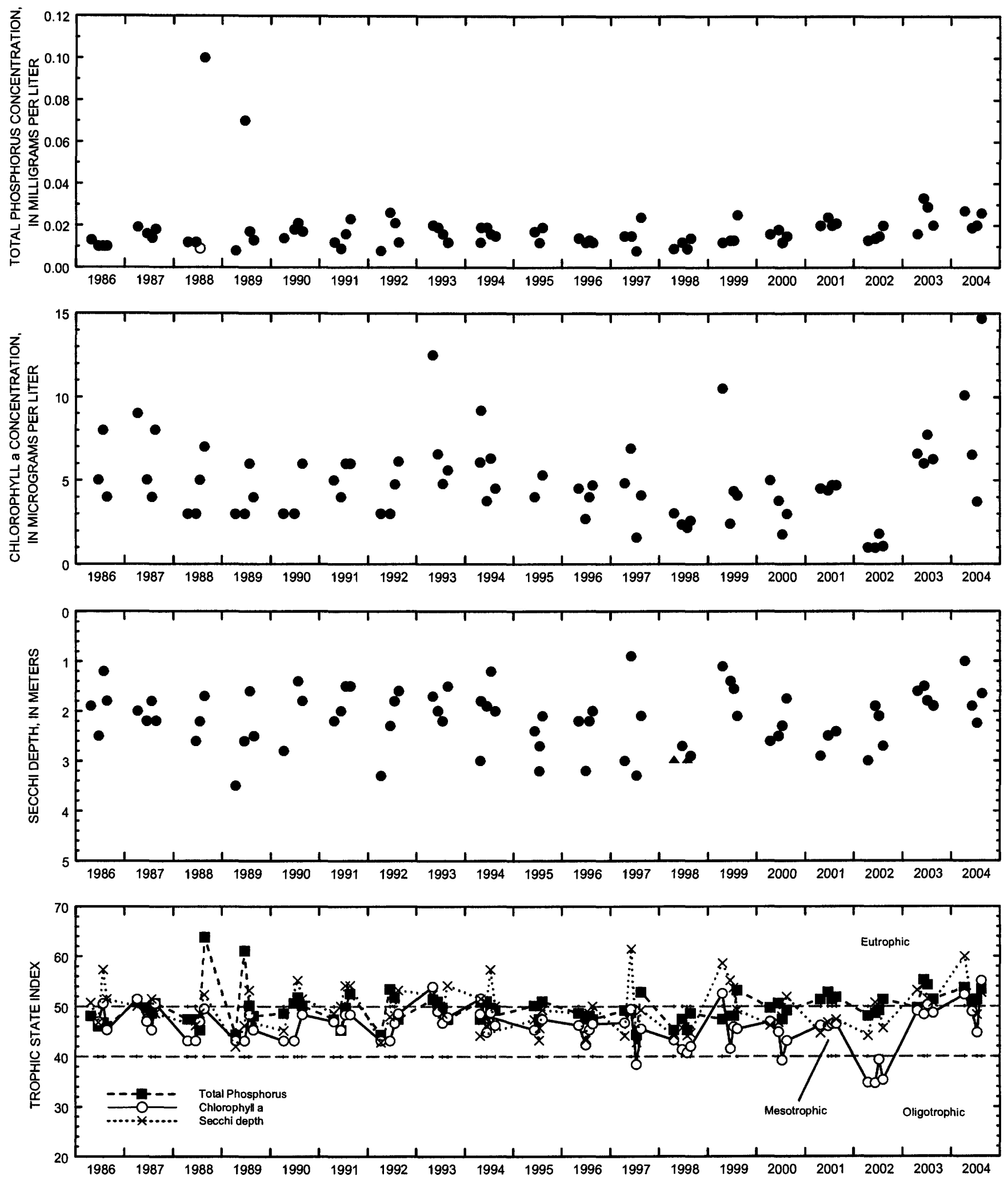

Surface total phosphorus, chlorophyll a concentrations, Secchi depths, and TSI data for Okauchee Lake, No. 2, near Okauchee, Wisconsin.

(Circles on the first two plots indicate laboratory detection limit for selected analyses. Actual concentrations for these particular analyses are less than the plotted circles.)

(Triangles in Secchi plot indicate maximum depth at sampling site.

Actual Secchi depth on these days was greater than the plotted triangles.) 


\section{OKAUCHEE LAKE, NO. 3, AT OKAUCHEE, WI}

LOCATION.--Lat $43^{\circ} 06^{\prime} 42^{\prime \prime}$, long $88^{\circ} 25^{\prime} 24^{\prime \prime}$, in NE $1 / 4$ SE $1 / 4 \mathrm{sec} .36$, T.8 N., R.17 E., Waukesha County, Hydrologic Unit 07090001 , at Okauchee.

PERIOD OF RECORD.--April 1986 to current year.

LAKE-STAGE GAGE.--Datum of gage is $869.00 \mathrm{ft}$ above sea level.

REMARKS.--Lake sampled in Ice House Bay, in the southern part of the lake, at an approximate depth of $4 \mathrm{~m}$. Water-quality analyses done by Wisconsin State Laboratory of Hygiene.

WATER-QUALITY DATA, APRIL 13 TO AUGUST 17, 2004

(Milligrams per liter unless otherwise indicated)

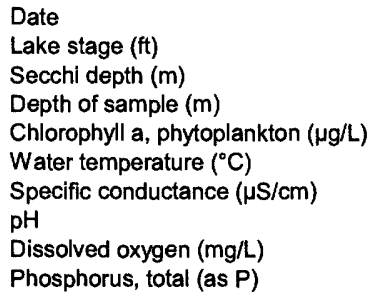

\begin{tabular}{cc} 
Apr-13 & Jun-8 \\
\hline 4.72 & 4.70 \\
1.2 & 1.7 \\
0.5 & 0.5 \\
11.1 & 6.9 \\
7.0 & 22.0 \\
553 & 545 \\
8.5 & 8.3 \\
13.2 & 11.1 \\
0.023 & 0.023
\end{tabular}

$\begin{array}{cc}\frac{J u l-15}{4.78} & \frac{\text { Aug-17 }}{4.7} \\ 1.4 & - \\ 0.5 & 0.5 \\ 9.7 & 12.4 \\ 25.1 & 21.3 \\ 540 & 538 \\ 8.1 & 8.3 \\ 9.5 & 11.7 \\ 0.022 & 0.021\end{array}$



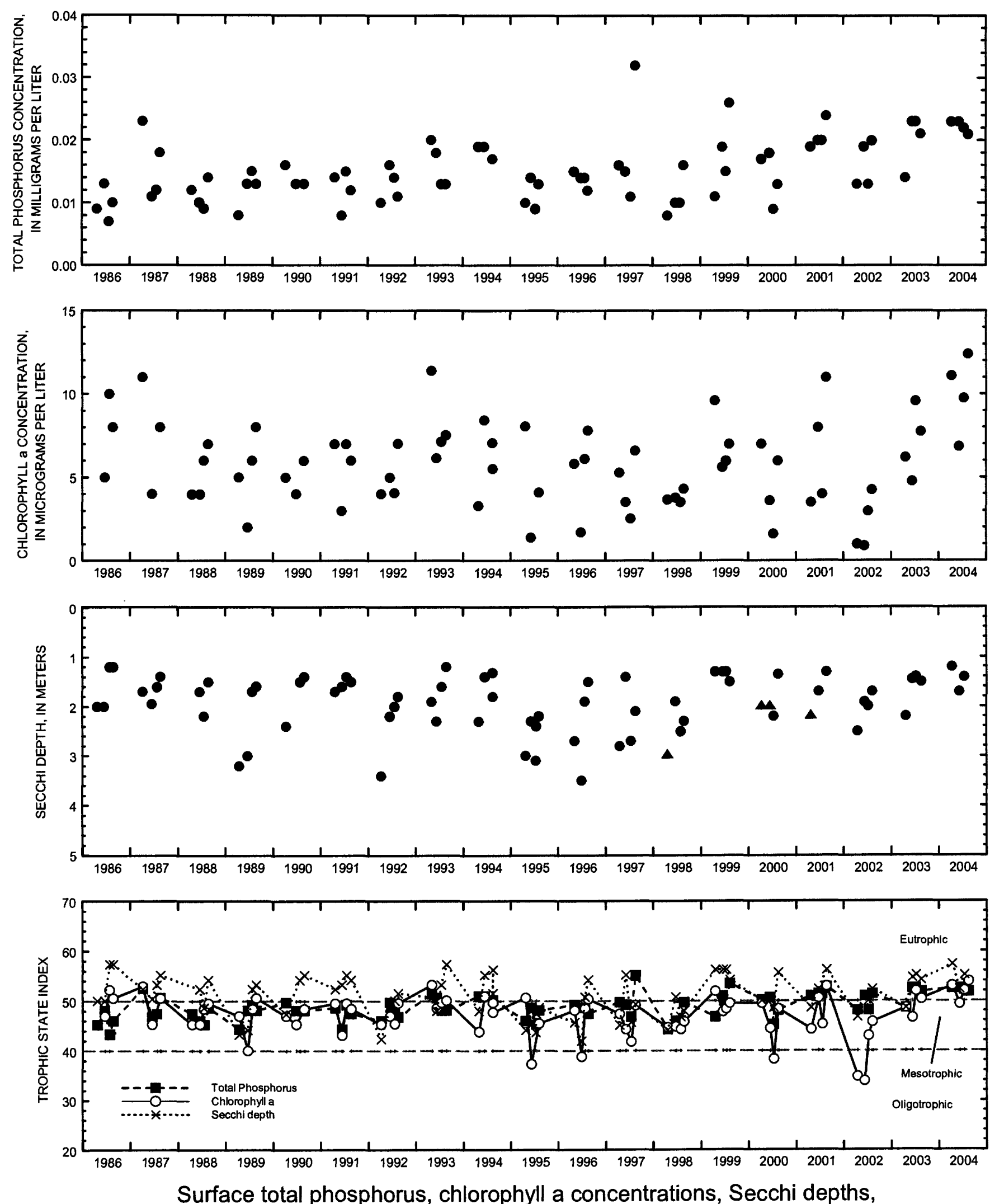

Surface total phosphorus, chlorophyll a concentrations, Secchi depths, and TSI data for Okauchee Lake, No. 3, near Okauchee, Wisconsin. 


\section{OKAUCHEE LAKE, NO. 4, AT OKAUCHEE, WI}

LOCATION.--Lat $43^{\circ} 07^{\prime} 57^{\prime \prime}$, long $88^{\circ} 26^{\prime} 17^{\prime \prime}$, in NW 1/4 NW 1/4 sec.25, T.8 N., R.17 E., Waukesha County, Hydrologic Unit 07090001, at Okauchee.

PERIOD OF RECORD.--June 1986 to current year.

LAKE-STAGE GAGE.--Datum of gage is $869.00 \mathrm{ft}$ above sea level.

REMARKS.--Lake sampled near McDowell (Crazyman's) Island, in the northwest bay of the lake, at an approximate depth of $2 \mathrm{~m}$. Waterquality analyses done by Wisconsin State Laboratory of Hygiene.

WATER-QUALITY DATA, APRIL 13 TO AUGUST 17, 2004

(Milligrams per liter unless otherwise indicated)

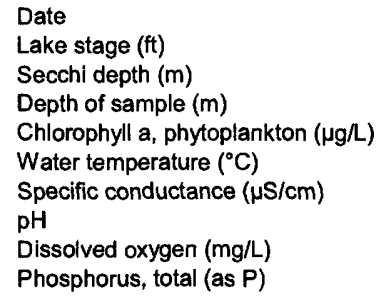

\begin{tabular}{c} 
Apr-13 \\
\hline 4.72 \\
1.4 \\
0.5 \\
8.7 \\
6.4 \\
546 \\
8.4 \\
13.7 \\
0.023
\end{tabular}



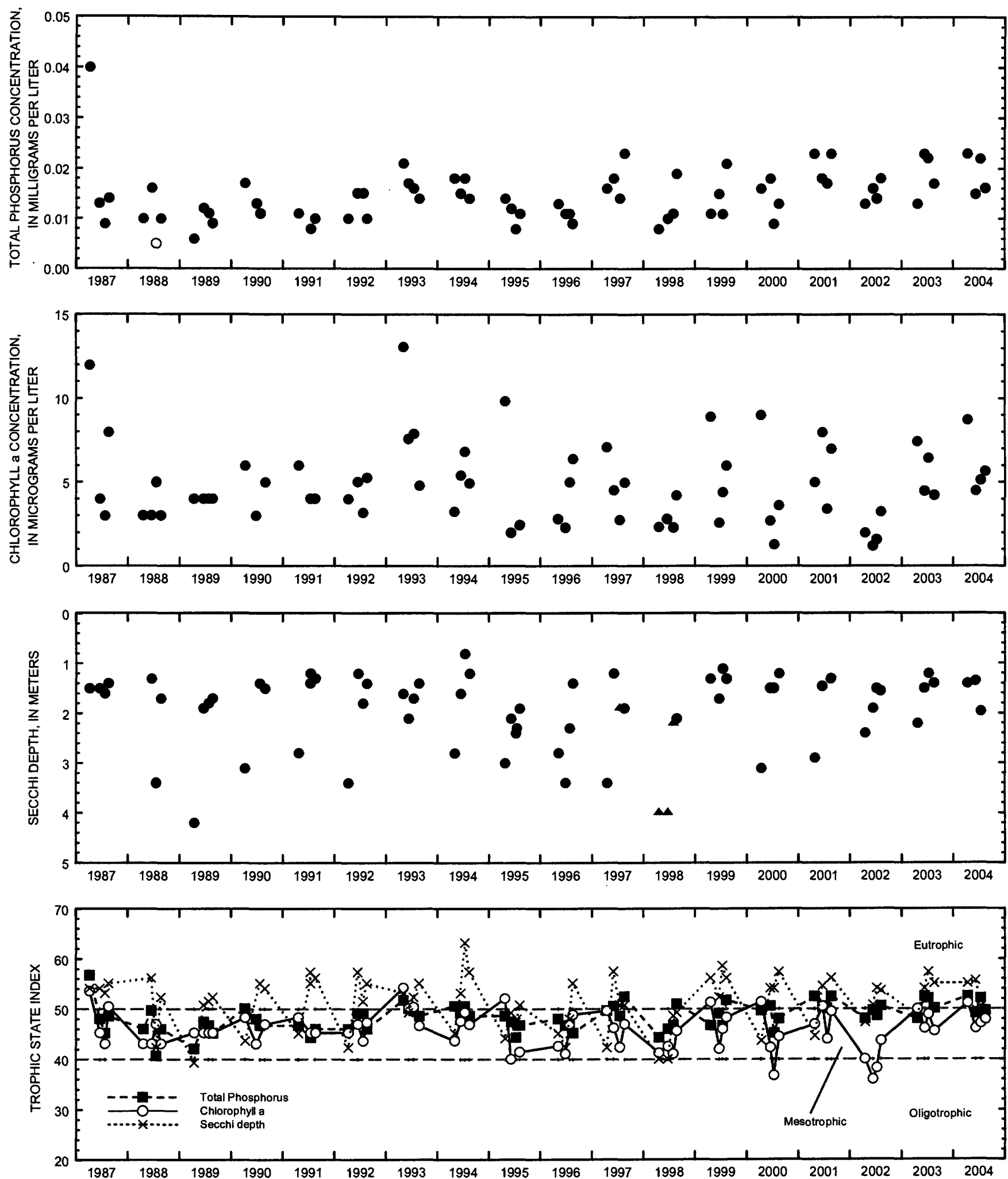

Surface total phosphorus, chlorophyll a concentrations, Secchi depths, and TSI data for Okauchee Lake, No. 4, near Okauchee, Wisconsin.

(Circles on the first two plots indicate laboratory detaction limit for selected anaiyses.

Actual concentrations for these particular analyses are less than the plottad circles.)

(Triangles in Secchi plot indicate maximum depth at sampling sita. Actual Secchi depth on these days was greatar than the plotted triangles.) 


\section{POTTER LAKE NEAR MUKWONAGO, WI}

LOCATION.--Lat $42^{\circ} 49^{\prime} 05^{\prime \prime}$, long $88^{\circ} 20^{\prime} 40^{\prime \prime}$, in NW 1/4 SW 1/4 sec.11, T.4 N., R.18 E., Walworth County, Hydrologic Unit $07120006,3.3$ mi south of Mukwonago.

PERIOD OF RECORD.--February 1993 to current year.

REMARKS.--Lake sampled at the deep hole. Lake ice-covered during February sampling. Water-quality analyses done by Wisconsin State Laboratory of Hygiene.

WATER-QUALITY DATA, APRIL 8 TO JULY 27, 2004

(Milligrams per liter unless otherwise indicated)

Date

Lake stage $(\mathrm{ft})$

Secchi depth $(\mathrm{m})$

Depth of sample $(\mathrm{m})$

Chlorophyll a, phytoplankton ( $\mu \mathrm{g} / \mathrm{L})$

Water temperature $\left({ }^{\circ} \mathrm{C}\right)$

Specific conductance $(\mu \mathrm{S} / \mathrm{cm})$

$\mathrm{pH}$

Dissolved oxygen (mg/L)

Phosphorus, total (as P)

Phosphorus, ortho, dissolved (as $\mathrm{P}$ )

Nitrogen, $\mathrm{NO}_{2}+\mathrm{NO}_{3}$, diss. (as $\mathrm{N}$ )

Nitrogen, ammonia, dissolved (as $\mathrm{N}$ )

Nitrogen, amm. + diss., total (as $\mathrm{N}$ )

Nitrogen, amm. + org., total (as N)

Color (Pt-Co. scale)

Turbidity (NTU)

Hardness, as $\mathrm{CaCO}_{3}$

Calcium, dissolved $(\mathrm{Ca})$

Magnesium, dissolved $(\mathrm{Mg})$

Sodium, dissolved ( $\mathrm{Na}$ )

Potassium, dissolved (K)

Alkalinity, as $\mathrm{CaCO}_{3}$

Sulfate, dissolved $\left(\mathrm{SO}_{4}\right)$

Chloride, dissolved (Cl)

Silica, dissolved $\left(\mathrm{SiO}_{2}\right)$

Solids, dissolved, at $180^{\circ} \mathrm{C}$

Iron, dissolved $(\mathrm{Fe})(\mu \mathrm{g} / \mathrm{L})$

Manganese, dissolved, $(\mathrm{Mn})(\mu \mathrm{g} / \mathrm{L})$

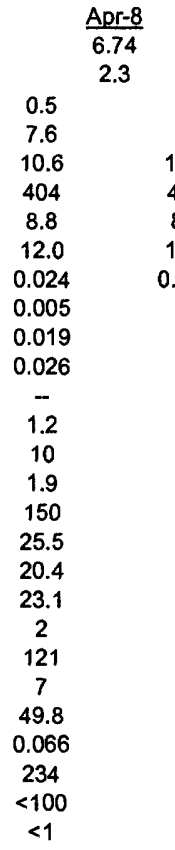

6-7-04

DISSOLVED OXYGEN (D.O.) IN MILLIGRAMS PER LITER

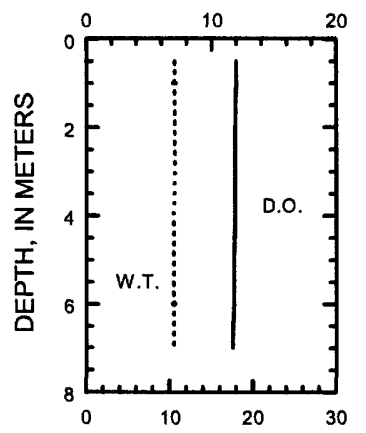

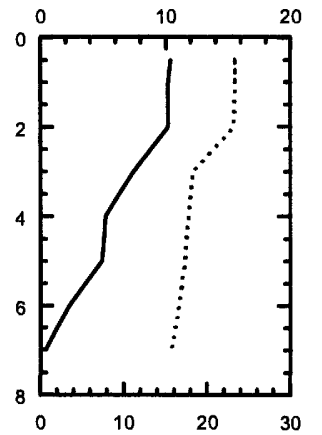

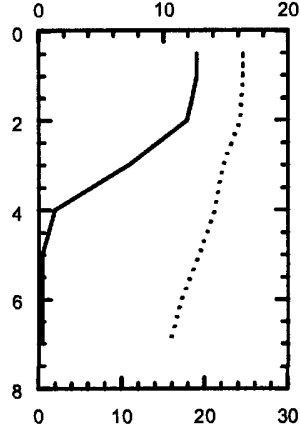

WATER TEMPERATURE (W.T.) IN DEGREES CELSIUS

\begin{tabular}{|c|c|c|c|}
\hline \multicolumn{2}{|c|}{$\frac{\mathrm{Jul}-12}{8.36}$} & \multicolumn{2}{|c|}{$\frac{\mathrm{Jul}-27}{8.08}$} \\
\hline 0.5 & 7 & 0.5 & 7 \\
\hline 16.2 & -- & 13.8 & - \\
\hline 24.6 & 15.7 & 23.6 & 16.0 \\
\hline 427 & 555 & 453 & 558 \\
\hline 8.3 & 7.2 & 7.9 & 7.1 \\
\hline 12.7 & 0.3 & 7.2 & 0.2 \\
\hline 0.037 & 0.369 & 0.042 & 0.263 \\
\hline 0.018 & - & - & - \\
\hline$<0.019$ & - & - & - \\
\hline 0.024 & - & - & - \\
\hline 0.89 & - & - & - \\
\hline - & -- & -- & - \\
\hline- & - & -- & - \\
\hline- & - & - & - \\
\hline - & - & - & - \\
\hline - & -- & - & -- \\
\hline- & - & - & - \\
\hline - & - & - & -- \\
\hline - & -- & - & - \\
\hline- & -- & - & - \\
\hline - & -- & -- & -- \\
\hline- & -- & - & - \\
\hline- & - & - & - \\
\hline- & - & - & -- \\
\hline - & - & - & - \\
\hline & - & - & -- \\
\hline
\end{tabular}
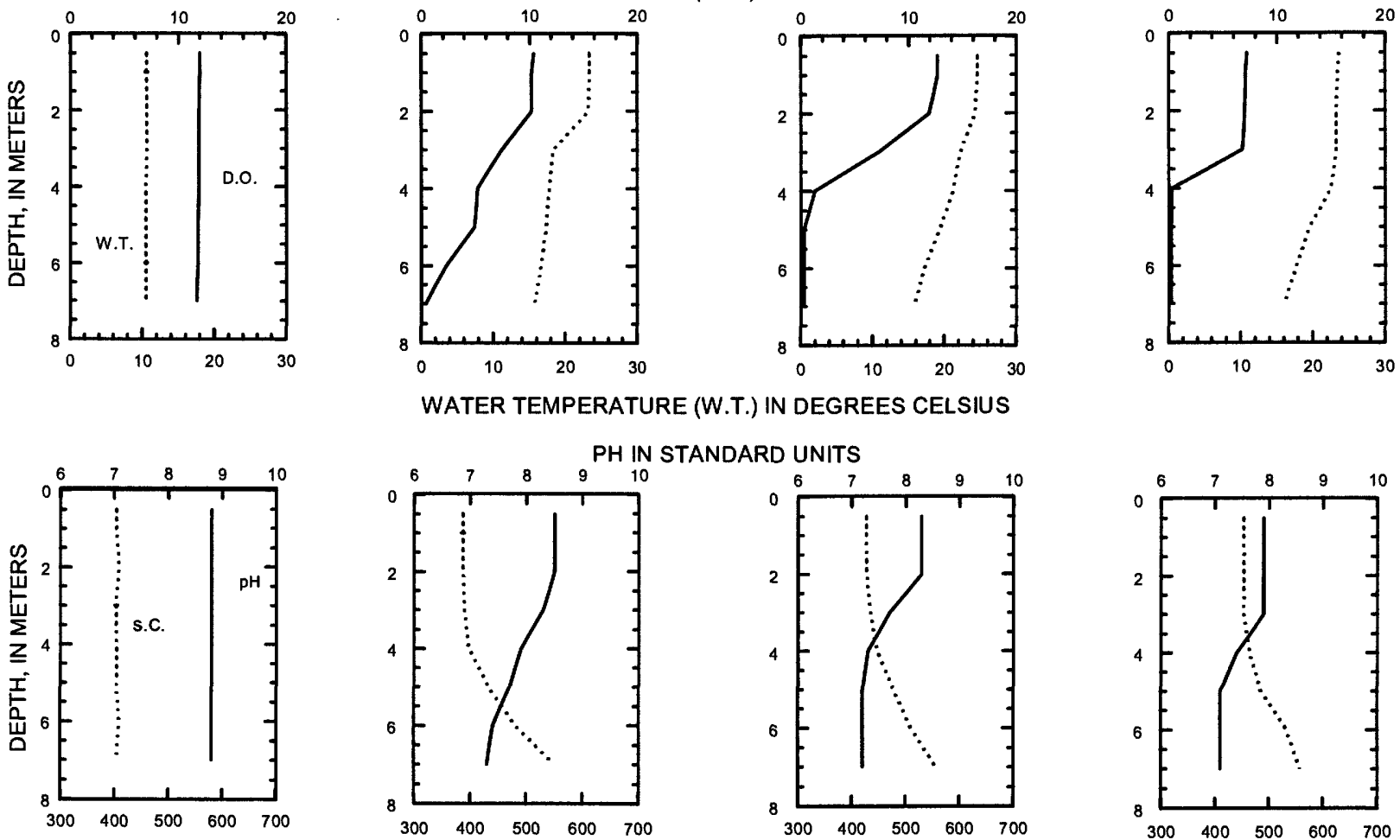

SPECIFIC CONDUCTANCE (S.C.) IN MICROSIEMENS PER CENTIMETER AT 25 DEGREES CELSIUS 
WATER-QUALITY DATA, AUGUST 9 TO SEPTEMBER 23, 2004

(Milligrams per liter unless otherwise indicated)

Date

Lake stage (ft)

Secchi depth $(\mathrm{m})$

Depth of sample $(m)$

Chlorophyll a, phytoplankton ( $\mu \mathrm{g} / \mathrm{L})$

Water temperature $\left({ }^{\circ} \mathrm{C}\right)$

Specific conductance $(\mu \mathrm{S} / \mathrm{cm})$

$\mathrm{pH}$

Dissolved oxygen ( $\mathrm{mg} / \mathrm{L})$

Phosphorus, total (as P)

$\begin{array}{cc} & \frac{\text { Aug-9 }}{8.06} \\ & 1.3 \\ 0.5 & 5 \\ 18.3 & -. \\ 24.3 & 19.5 \\ 455 & 507 \\ 7.8 & 7.8 \\ 8.3 & 0.2 \\ 0.049 & 0.064\end{array}$

8-24-04

DISSOLVED OXYGEN (D.O.) IN MILLIGRAMS PER LITER

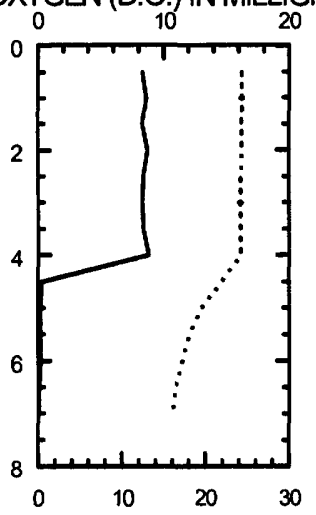

WATER TEMPERATURE (W.T.) INDEGREES CELSIUS

PH IN STANDARD UNITS

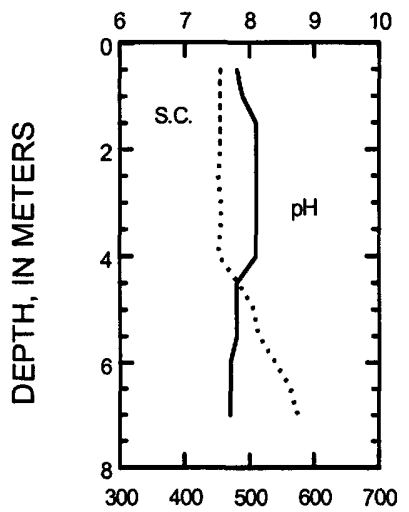

SPECIFIC CONDUCTANCE (S.C.) IN MICROSIEMENS PER CENTIMETER AT 25 DEGREES CELSIUS

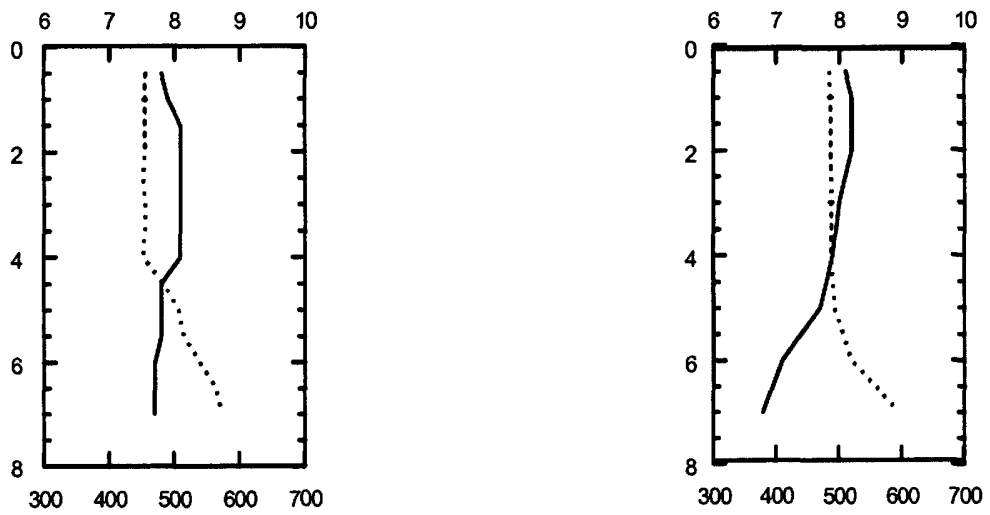

$9-23-04$

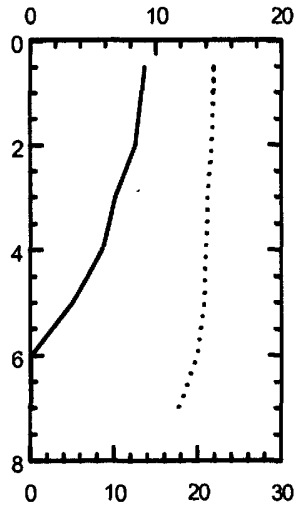



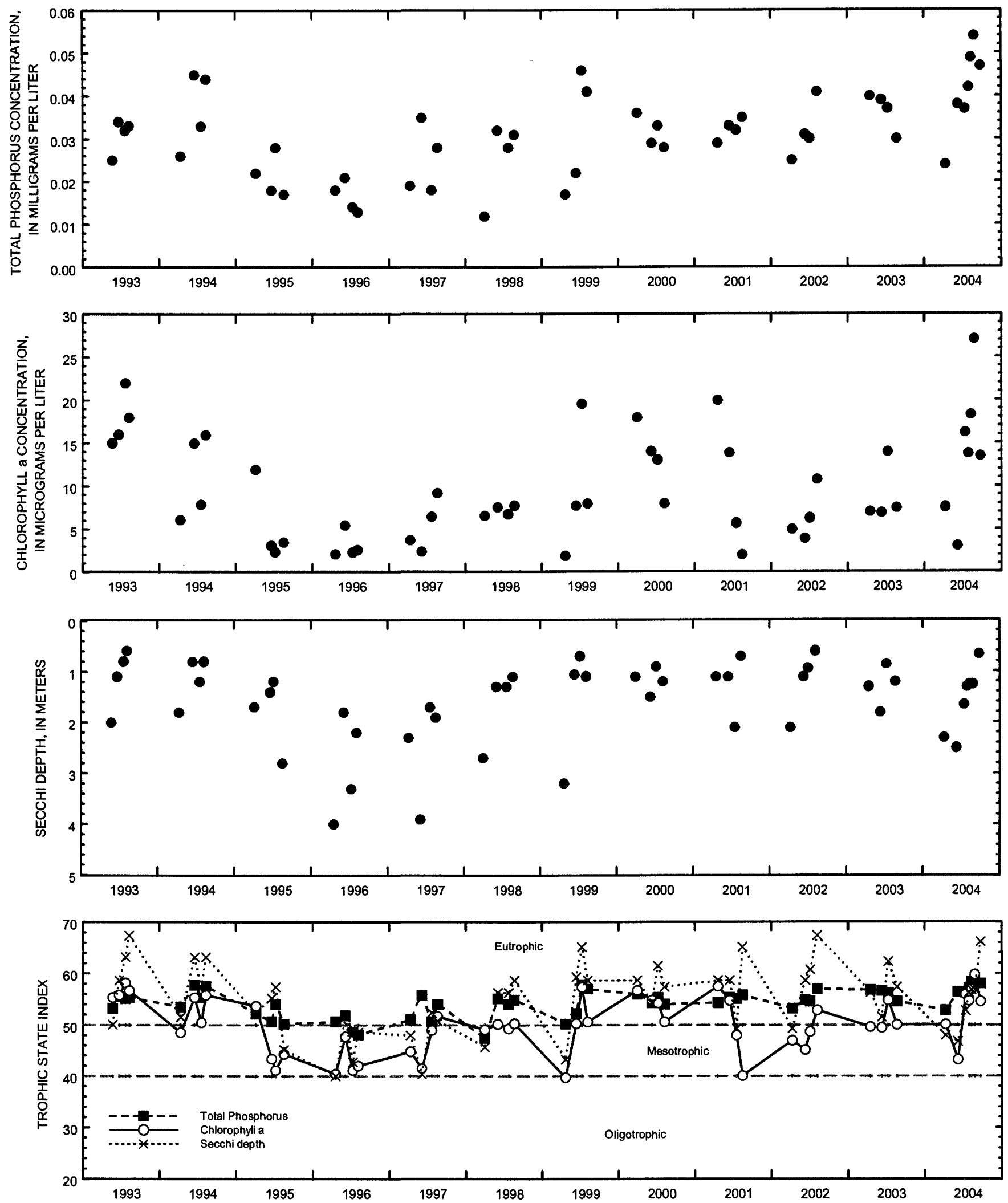

Surface total phosphorus, chlorophyll a concentrations, Secchi depths, and TSI data for Potter Lake, near Mukwonago, Wisconsin. 


\section{POWERS LAKE AT POWERS LAKE, WI}

LOCATION.--Lat $42^{\circ} 32^{\prime} 46^{\prime \prime}$, long $88^{\circ} 17^{\prime} 58^{\prime \prime}$, in NW 1/4 SE 1/4 sec.13, T.1 N., R.18 E., Walworth County, Hydrologic Unit 07120006, at Powers Lake.

DRAINAGE AREA.--3.42 $\mathrm{mi}^{2}$.

PERIOD OF RECORD.--March 1986 to August 1996, and April 1998 to current year.

REMARKS.--Lake sampled near center at the deep hole. Lake ice-covered during February sampling. Water-quality analyses done by Wisconsin State Laboratory of Hygiene.

WATER-QUALITY DATA, FEBRUARY 25 TO JUNE 7, 2004

(Milligrams per liter unless otherwise indicated)

Date

Lake stage (ft)

Secchi depth $(m)$

Depth of sample (m)

Chlorophyll a, phytoplankton $(\mu \mathrm{g} / \mathrm{L})$

Water temperature $\left({ }^{\circ} \mathrm{C}\right)$

Specific conductance $(\mu \mathrm{S} / \mathrm{cm})$

$\mathrm{pH}$

Dissolved oxygen (mg/L)

Phosphorus, total (as P)

Phosphorus, ortho, dissolved (as P)

Nitrogen, $\mathrm{NO}_{2}+\mathrm{NO}_{3}$, diss. (as $\mathrm{N}$ )

Nitrogen, ammonia, dissolved (as $\mathrm{N}$ )

Nitrogen, amm. + org., total (as $\mathrm{N}$ )

Color (Pt-Co. scale)

Turbidity (NTU)

Hardness, as $\mathrm{CaCO}_{3}$

Calcium, dissolved $(\mathrm{Ca})$

Magnesium, dissolved (Mg)

Sodium, dissolved $(\mathrm{Na})$

Potassium, dissolved (K)

Alkalinity, as $\mathrm{CaCO}_{3}$

Sulfate, dissolved $\left(\mathrm{SO}_{4}\right)$

Chlonide, dissolved $(\mathrm{Cl})$

Silica, dissolved $\left(\mathrm{SiO}_{2}\right)$

Solids, dissolved, at $180^{\circ} \mathrm{C}$

Iron, dissolved $(\mathrm{Fe})(\mu \mathrm{g} / \mathrm{L})$

Manganese, dissolved, $(\mathrm{Mn})(\mu \mathrm{g} / \mathrm{L})$

\section{2-25-04}
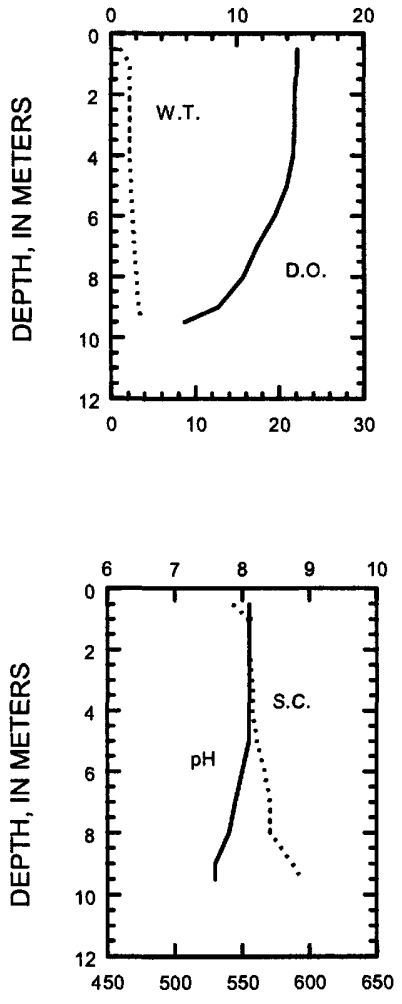

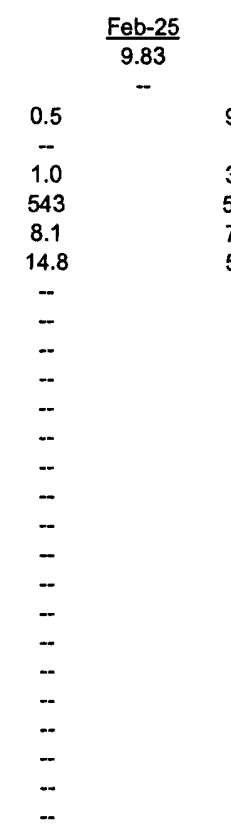

4-8-04
Jun-7

0.5

2.2

9.1

493

8.2
12.1

12.1

0.014
$<0.002$

$<0.002$
$<0.019$

0.009

0.6

0.6
10
1.3

1.3

210

32.4

32.1

19.4

2

168

30

40.2

5.24

280

$<100$

$<1$

10
-
8.5
495
8.2
11.2
0.014
-
-
-
-
-
-
-
-
-
-
-
-
-
-
-
-
-
-

DISSOLVED OXYGEN (D.O.) IN MILLIGRAMS PER LITER

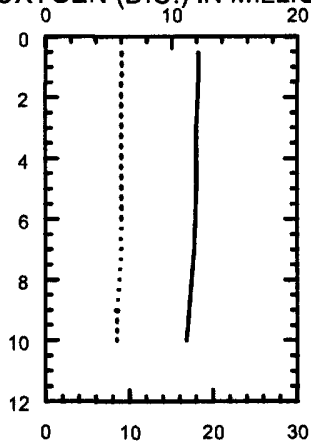

WATER TEMPERATURE (W.T.) IN DEGREES CELSIUS
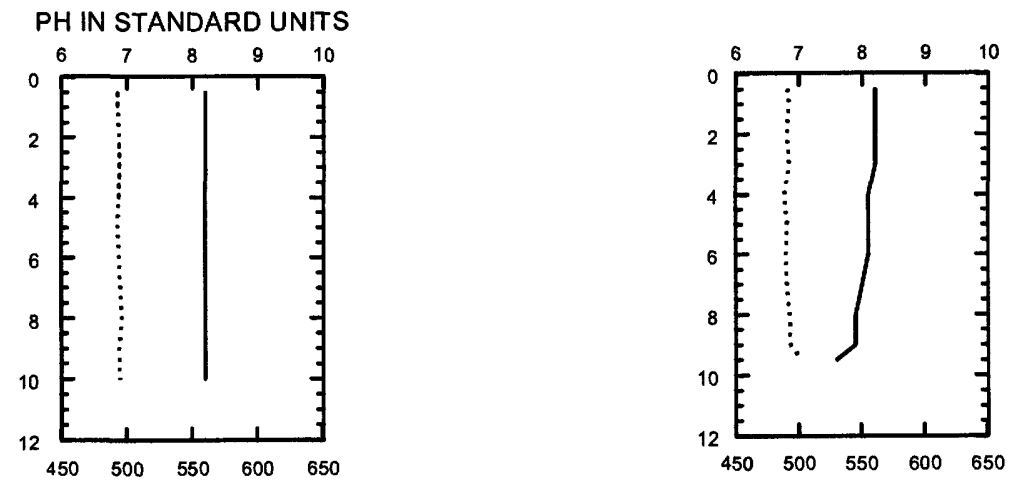
WATER-OUALITY DATA, JULY 12 TO SEPTEMBER 23, 2004

(Milligrams per liter unless otherwise indicated)

Date

Lake stage (ft)

Secchi-depth (m)

Depth of sample (m)

Chlorophyll a, phytoplankton ( $\mu \mathrm{g} / \mathrm{L}$ )

Water temperature $\left({ }^{\circ} \mathrm{C}\right)$

Specific conductance $(\mu \mathrm{S} / \mathrm{cm})$

Dissolved oxygen ( $\mathrm{mg} / \mathrm{L}$ )

Phosphorus, total (as P)

$7-12-04$
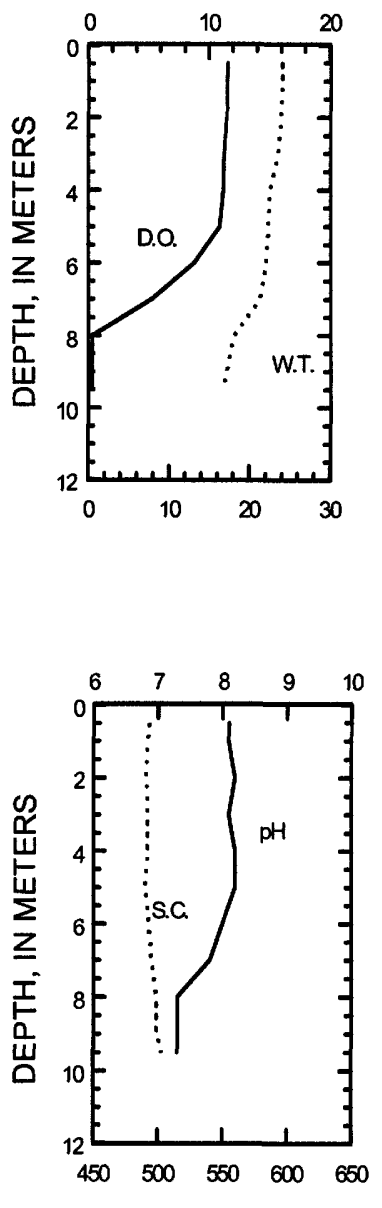

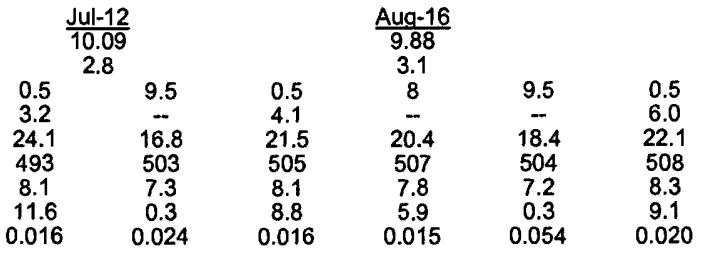

8-16-04

DISSOLVED OXYGEN (D.O.) IN MUGRAMS PERUTER

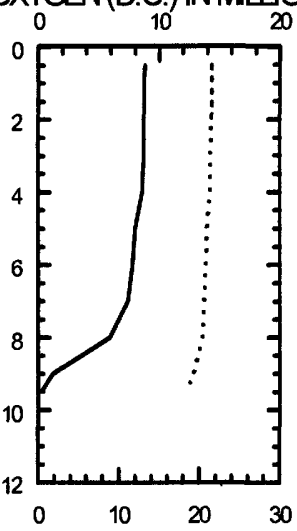

WATER TEMPERATURE (W.T.) INDEGREES CELSIUS

\section{PHINSTANDARD UNITS}

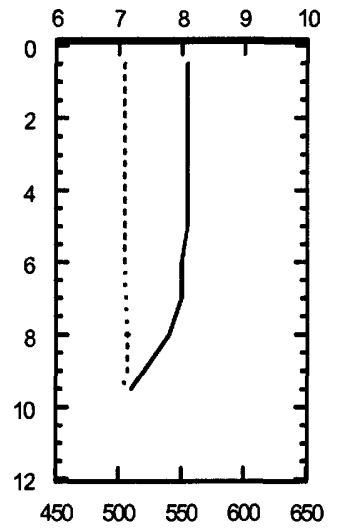

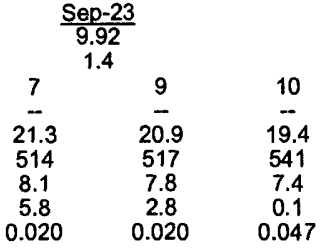

$9-23-04$
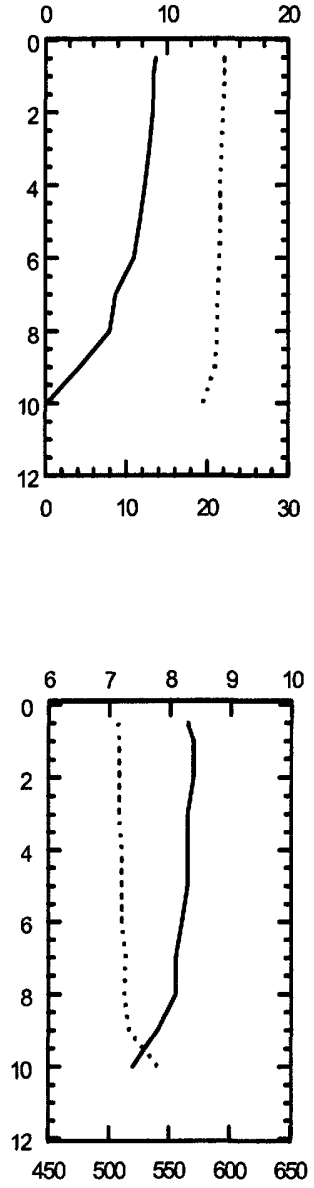

SPECIFIC CONDUCTANCE (S.C.) IN MICROSIEMENS PER CENTIMETERAT 25 DEGREES CELSIUS 

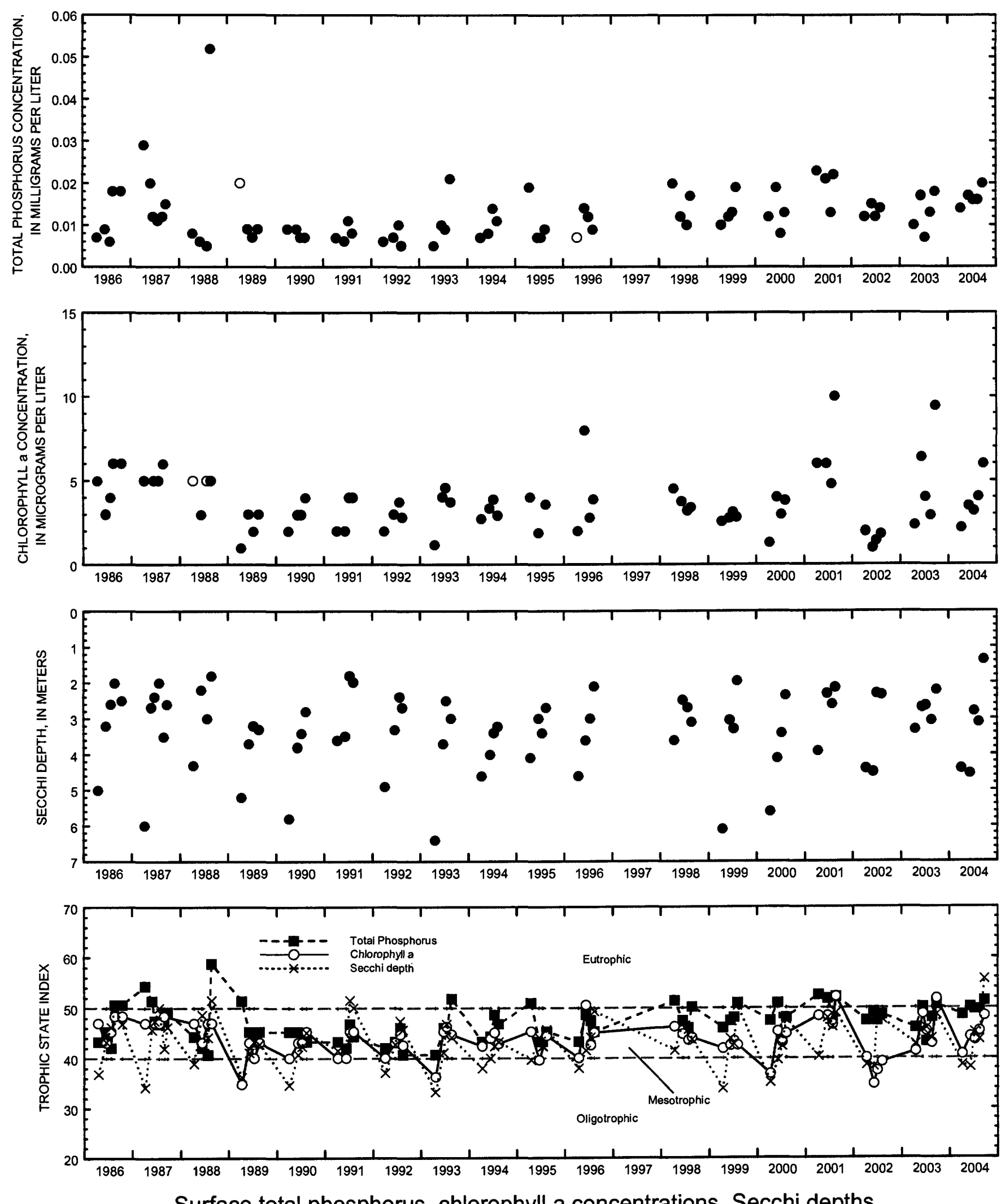

Surface total phosphorus, chlorophyll a concentrations, Secchi depths, and TSI data for Powers Lake, at Powers Lake, Wisconsin.

(Circles on the first two plots indicate laboratory detection iimit for selected analyses.

Actual concentrations for these particular analyses are less than the plotted circles.) 


\section{SPOONER LAKE, AT DEEP HOLE, NEAR SPOONER, WI}

LOCATION.--Lat $45^{\circ} 50^{\prime} 34 "$, long 91 $49^{\prime} 33 "$, in NE 1/4 NE 1/4 sec.27, T.39 N., R.12 W., Washburn County, Hydrologic Unit 07030001, near Spooner.

PERIOD OF RECORD.--June 2002 to current year.

REMARKS.--Lake sampled at deepest hole. Lake ice-covered during March sampling. Water-quality analyses by Wisconsin State Laboratory of Hygiene.

WATER-QUALITY DATA, JUNE 9 TO JULY 12, 2004

(Milligrams per liter unless otherwise indicated)

Date

Lake stage (ft)

Secchi depth (m)

Depth of sample (m)

Chlorophyll a, phytoplankton ( $\mu \mathrm{g} / \mathrm{L})$

Water temperature $\left({ }^{\circ} \mathrm{C}\right)$

Specific conductance $(\mu \mathrm{S} / \mathrm{cm})$

$\mathrm{pH}$

Dissolved oxygen $(\mathrm{mg} / \mathrm{L})$

Phosphorus, total (as P)

$\begin{array}{ccc} & \text { Jun-9 } & \\ & 7.08 & \\ & 1.9 & \\ 0.5 & & 4.5 \\ 9.1 & & - \\ 22.0 & & 21.0 \\ 179 & & 185 \\ 8.0 & & 7.6 \\ 9.1 & & 5.5 \\ 0.035 & & 0.031\end{array}$

$6-21-04$

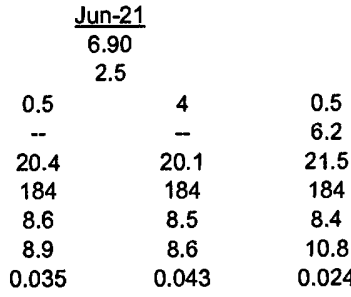

$7-2-04$

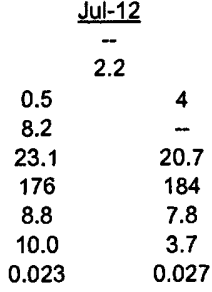

$7-12-04$

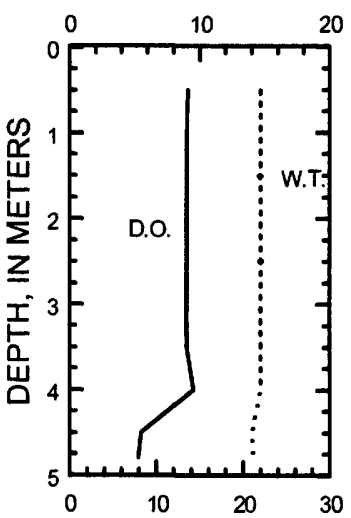

DISSOLVED OXYGEN (D.O.) IN MILLIGRAMS PER LITER
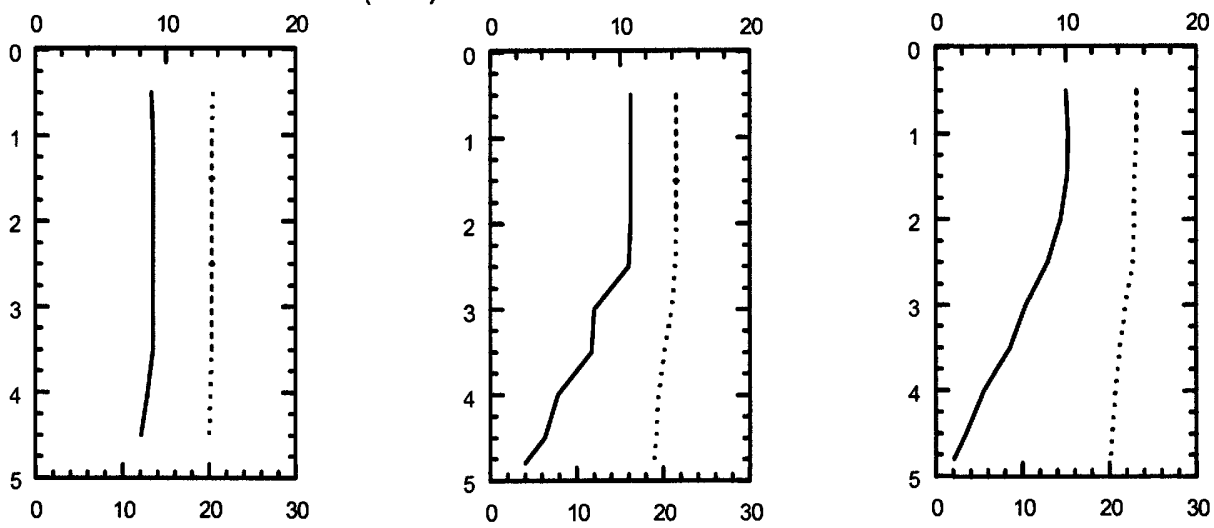

WATER TEMPERATURE (W.T.) IN DEGREES CELSIUS
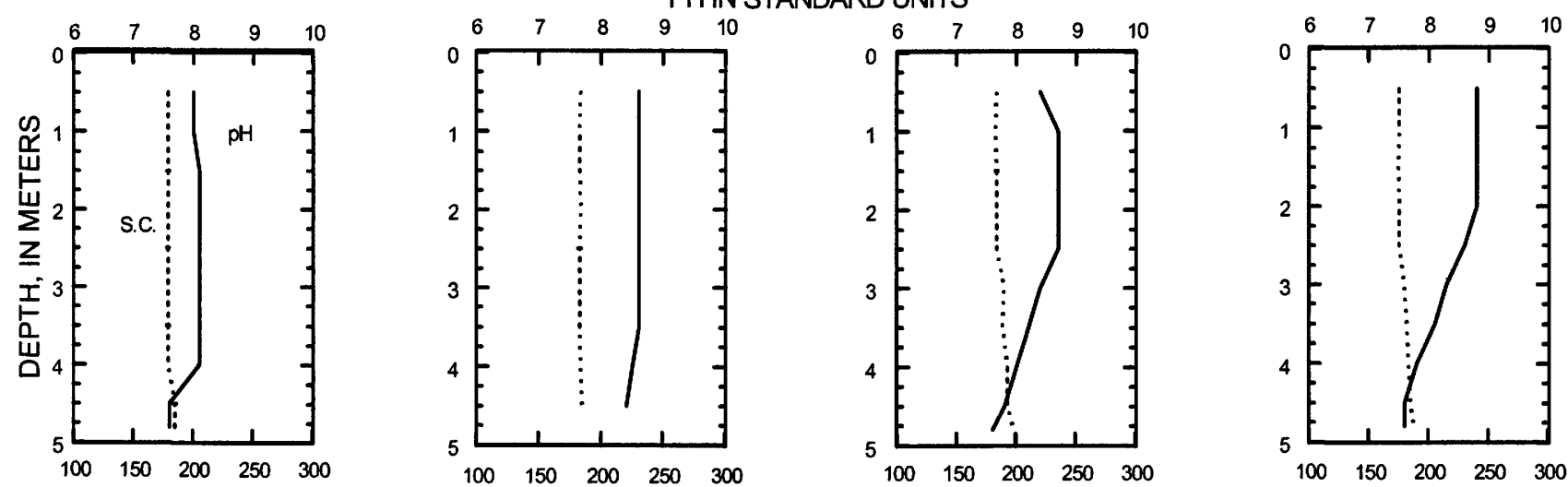

SPECIFIC CONDUCTANCE (S.C.) IN MICROSIEMENS PER CENTIMETER AT 25 DEGREES CELSIUS 
WATER-QUALITY DATA, JULY 20 TO AUGUST 25, 2004

(Milligrams per liter unless otherwise indicated)

Date

Lake stage (ft)

Secchi depth $(\mathrm{m})$

Depth of sample $(\mathrm{m})$

Chiorophyll a, phytoplankton $(\mu g / L)$

Water temperature $\left({ }^{\circ} \mathrm{C}\right)$

Specific conductance $(\mu \mathrm{S} / \mathrm{cm})$

$\mathrm{pH}$

Dissolved oxygen (mg/L)

Phosphorus, total (as P)

Phosphorus, ortho, dissolved (as $\mathrm{P}$ )

Nitrogen, $\mathrm{NO}_{2}+\mathrm{NO}_{3}$, diss. (as $\mathrm{N}$ )

Nitrogen, ammonia, dissolved (as $\mathrm{N}$ )

Nitrogen, amm. + diss., total (as $\mathrm{N}$ )

\begin{tabular}{|c|c|c|c|c|}
\hline \multicolumn{2}{|c|}{ Jul-20 } & \multicolumn{2}{|c|}{ Jul-28 } & \\
\hline \multicolumn{2}{|c|}{7.02} & \multicolumn{2}{|c|}{6.95} & \\
\hline \multicolumn{2}{|c|}{1.9} & \multicolumn{2}{|c|}{1.7} & \\
\hline 0.5 & 4.5 & 0.5 & 4 & 0.5 \\
\hline 8.1 & - & 8.8 & - & 8.5 \\
\hline 25.8 & 23.5 & 24.5 & 24.3 & 17.9 \\
\hline 169 & 187 & 169 & 169 & 153 \\
\hline 8.8 & 8.0 & 8.7 & 8.8 & 7.7 \\
\hline 9.9 & 0.7 & 8.9 & 8.8 & 8.6 \\
\hline 0.025 & 0.031 & 0.033 & 0.033 & 0.028 \\
\hline -- & -- & 0.003 & - & $\cdots$ \\
\hline -- & -- & $<0.019$ & - & - \\
\hline- & -- & $<0.015$ & - & - \\
\hline- & - & 0.52 & - & - \\
\hline
\end{tabular}

Aug-12
7.12
3.0
3.5
-
17.1
153
8.7
8.2
0.028
-
-
-
-

8-12-04

$8-25-04$

DISSOLVED OXYGEN (D.O.) IN MILIGRAMS PERLITER
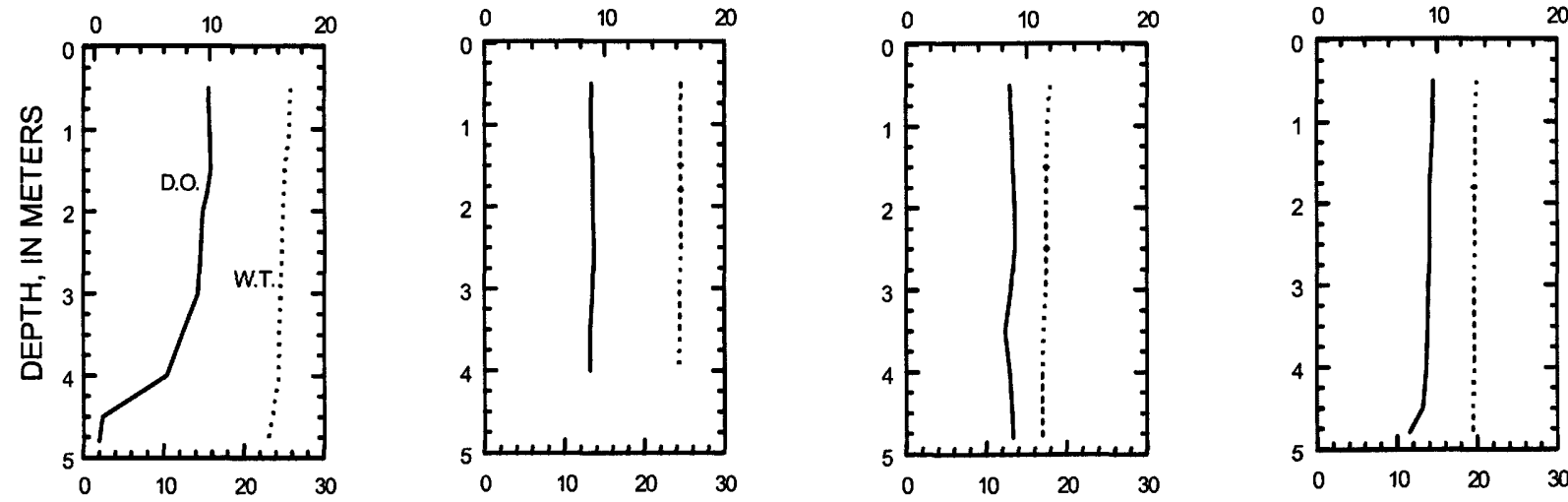

WATER TEMPERATURE (W.T.) IN DEGREES CELSIUS
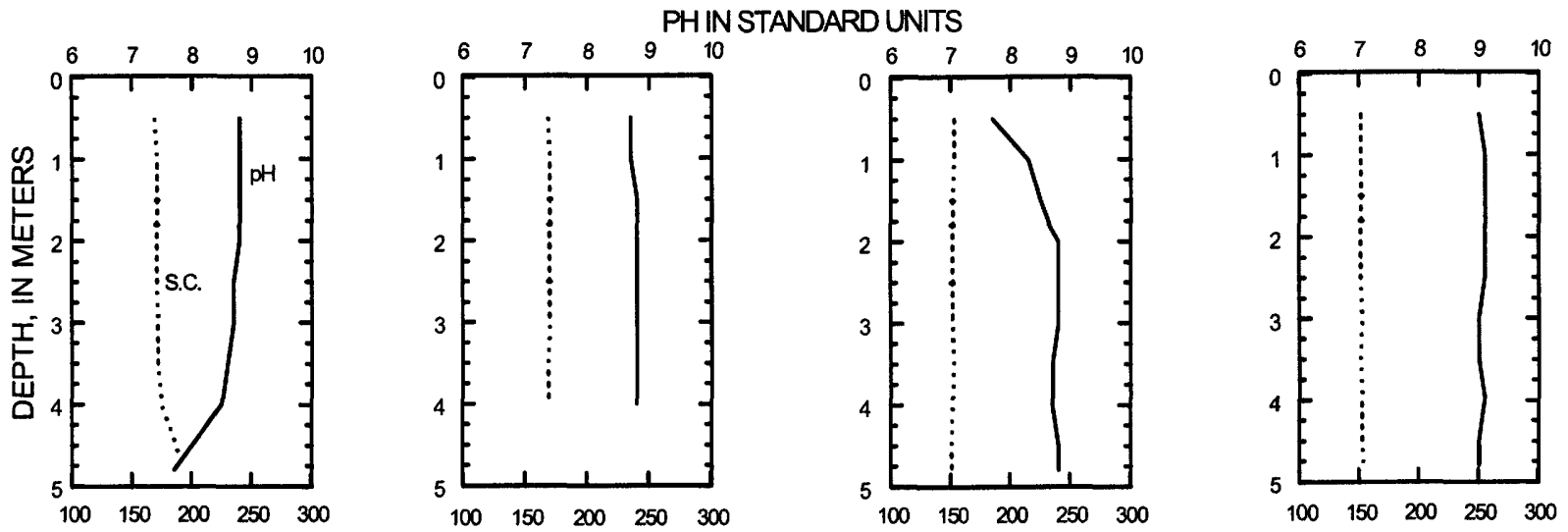

SPECIFIC CONDUCTANCE (S.C.) IN MICROSIEMENS PER CENTIMETER AT 25 DEGREES CELSIUS 

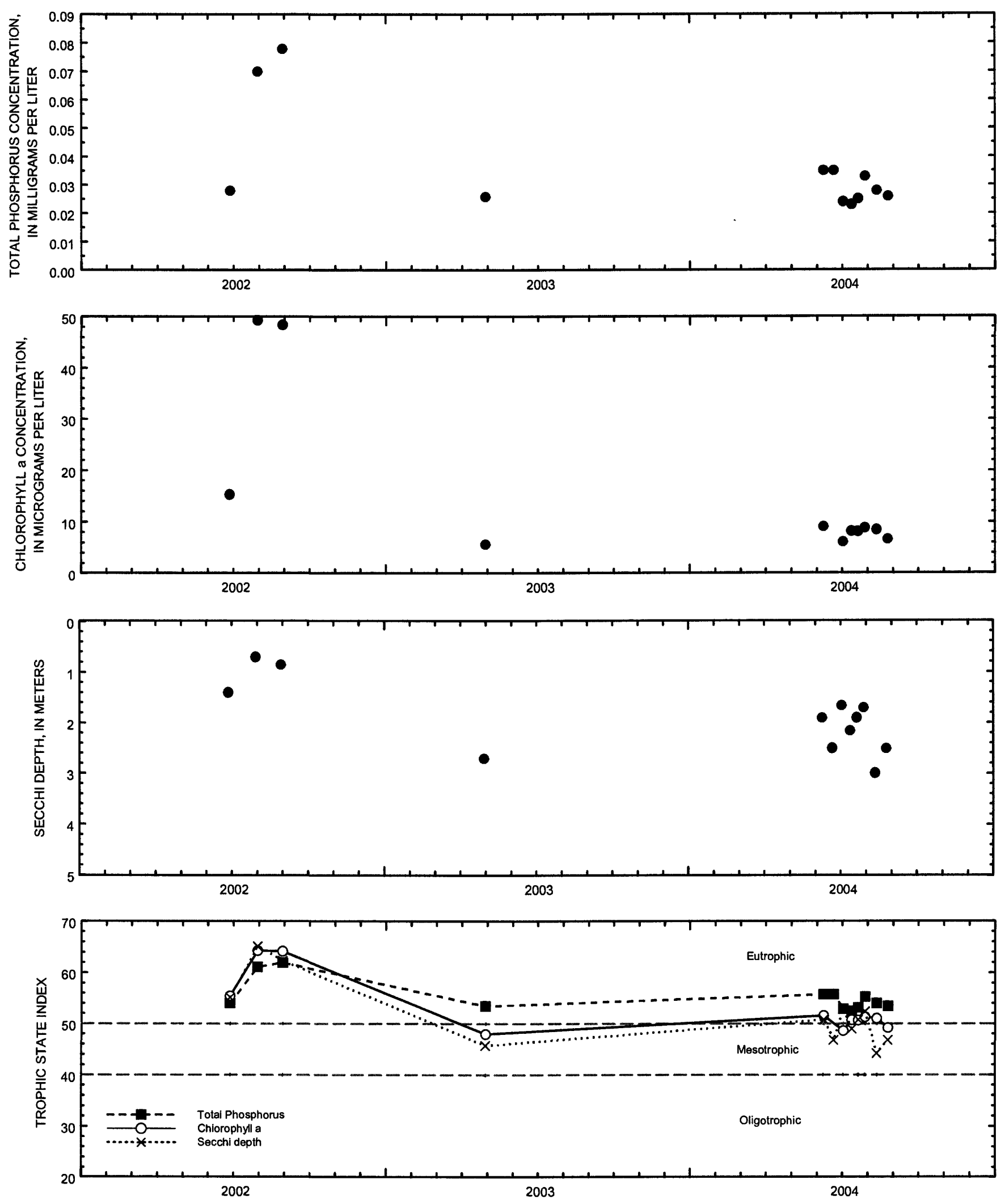

Surface total phosphorus, chlorophyll a concentrations, Secchi depths, and TSI data for Spooner Lake, Deep Hole, near Spooner, Wisconsin. 
LOCATION.--Lat $45^{\circ} 49^{\prime} 45^{\prime \prime}$, long 91 48'39", in SW 1/4 SE 1/4 sec.26, T.39 N., R.12 W., Washburn County, Hydrologic Unit 07030001, near Spooner.

PERIOD OF RECORD.--June 2002 to current year.

REMARKS.--Lake sampled at deepest hole. Lake ice-covered during March sampling. Water-quality analyses by Wisconsin State Laboratory of Hygiene.

WATER-QUALITY DATA, JUNE 9 TO JULY 12, 2004

(Milligrams per liter unless otherwise indicated)

Date

Lake stage ( $\mathrm{ft}$ )

Secchi depth $(\mathrm{m})$

Depth of sample (m)

Chlorophyll a, phytoplankton $(\mu \mathrm{g} / \mathrm{L})$

Water temperature $\left({ }^{\circ} \mathrm{C}\right)$

Specific conductance $(\mu \mathrm{S} / \mathrm{cm})$

$\mathrm{pH}$

Dissolved oxygen (mg/L)

Phosphorus, total (as P)

\begin{tabular}{|c|c|c|c|c|c|c|c|}
\hline \multicolumn{2}{|c|}{ Jun-9 } & \multicolumn{2}{|c|}{ Jun-21 } & \multicolumn{2}{|c|}{ Jul-2 } & \multicolumn{2}{|c|}{ Jul-12 } \\
\hline \multicolumn{2}{|c|}{7.08} & \multicolumn{2}{|c|}{6.90} & \multicolumn{2}{|c|}{$\overline{7.08}$} & \multicolumn{2}{|c|}{7.10} \\
\hline \multicolumn{2}{|c|}{1.9} & \multicolumn{2}{|c|}{2.3} & \multicolumn{2}{|c|}{2.4} & \multicolumn{2}{|c|}{2.0} \\
\hline 0.5 & 2 & 0.5 & 2.2 & 0.5 & 2 & 0.5 & 2 \\
\hline 7.6 & -- & 6.2 & - & 8.1 & - & 12.2 & - \\
\hline 22.2 & 22.2 & 20.6 & 20.5 & 22.3 & 22.0 & 23.5 & 21.1 \\
\hline 177 & 177 & 190 & 190 & 191 & 195 & 194 & 206 \\
\hline 8.5 & 8.5 & 8.3 & 8.4 & 8.8 & 8.6 & 8.4 & 7.7 \\
\hline 9.9 & 10.1 & 8.3 & 8.4 & 11.7 & 9.9 & 9.6 & 6.8 \\
\hline 0.037 & 0.036 & 0.058 & 0.050 & 0.034 & 0.033 & 0.040 & 0.037 \\
\hline
\end{tabular}

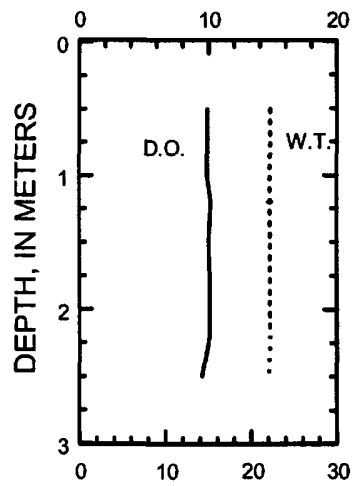

DISSOLVED OXYGEN (D.O.) IN MILLIGRAMS PER LITER
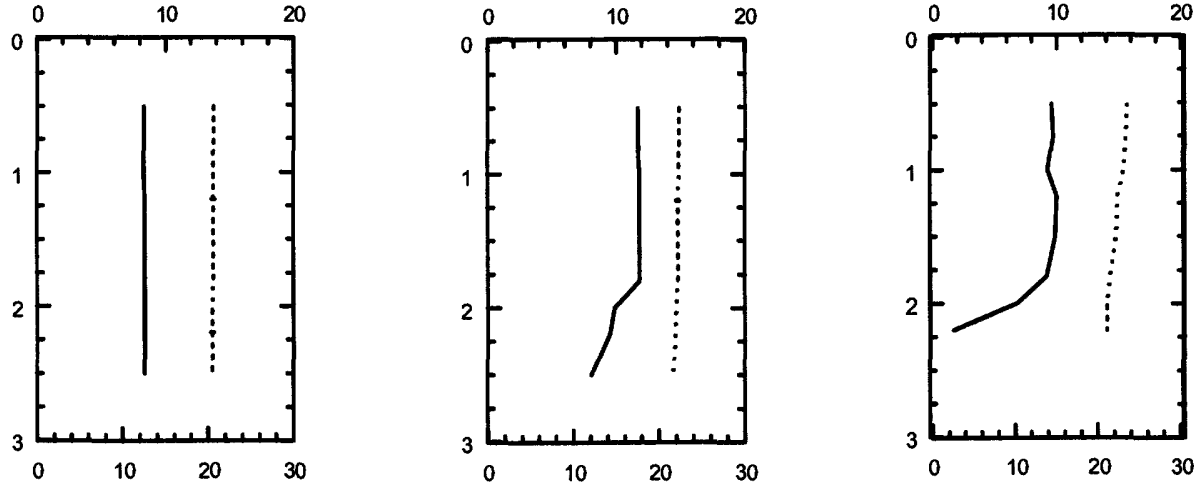

WATER TEMPERATURE (W.T.) IN DEGREES CELSIUS
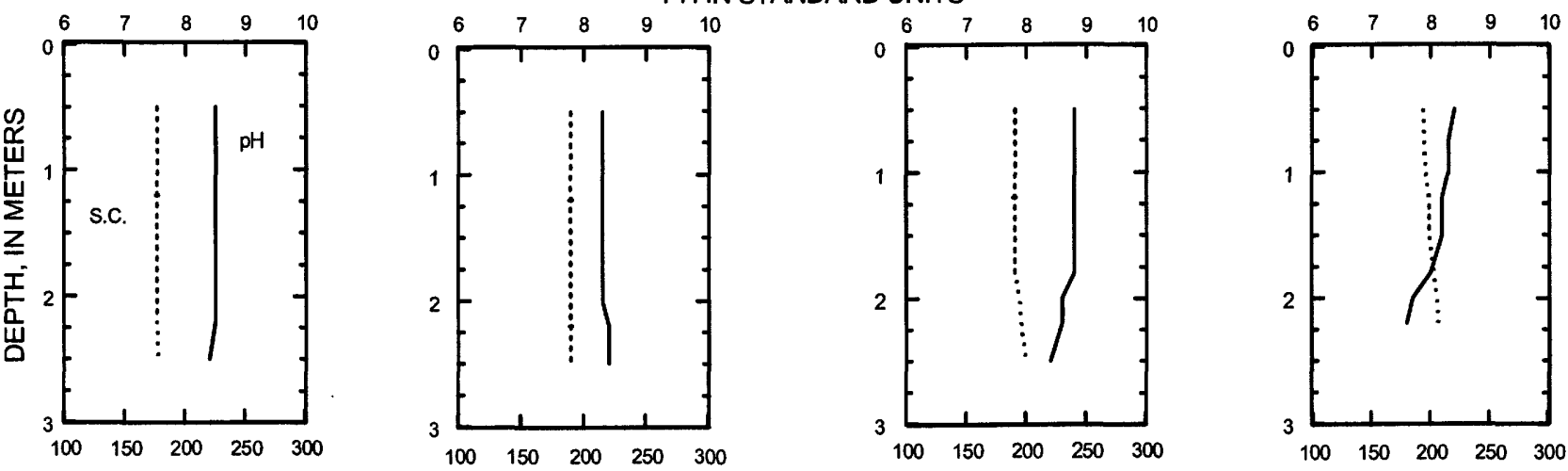

SPECIFIC CONDUCTANCE (S.C.) IN MICROSIEMENS PER CENTIMETER AT 25 DEGREES CELSIUS 
WATER-QUALITY DATA, JULY 20 TO AUGUST 25, 2004

(Milligrams per liter unless otherwise indicated)

Date

Lake stage (ft)

Secchi depth $(\mathrm{m})$

Depth of sample $(\mathrm{m})$

Chlorophyll e, phytoplankton $(\mu g / L)$

Water temperature $\left({ }^{\circ} \mathrm{C}\right)$

Specific conductance $(\mu \mathrm{S} / \mathrm{cm})$

$\mathrm{pH}$

Dissolved oxygen (mg/L)

Phosphorus, total (as P)

\begin{tabular}{|c|c|c|}
\hline & $\begin{array}{c}\frac{\mathrm{Ju} \mid-20}{7.02} \\
1.6\end{array}$ & \\
\hline 0.5 & & 2 \\
\hline 16.5 & & - \\
\hline 26.0 & & 23.7 \\
\hline 197 & & 206 \\
\hline 8.5 & & 8.0 \\
\hline 10.0 & & 8.3 \\
\hline 0.043 & & 0.0 \\
\hline
\end{tabular}

\begin{tabular}{ccc} 
& \multicolumn{3}{c}{$\frac{\text { Jul-28 }}{6.95}$} & \\
& 1.6 & \\
0.5 & & 2 \\
19.6 & & -- \\
23.6 & & 23.6 \\
201 & & 201 \\
8.4 & & 8.4 \\
9.1 & & 9.2 \\
0.044 & & 0.041
\end{tabular}

7-28-04

8-12-04

8-25-04

DISSOLVED OXYGEN (D.O.) IN MILLIGRAMS PER LITER
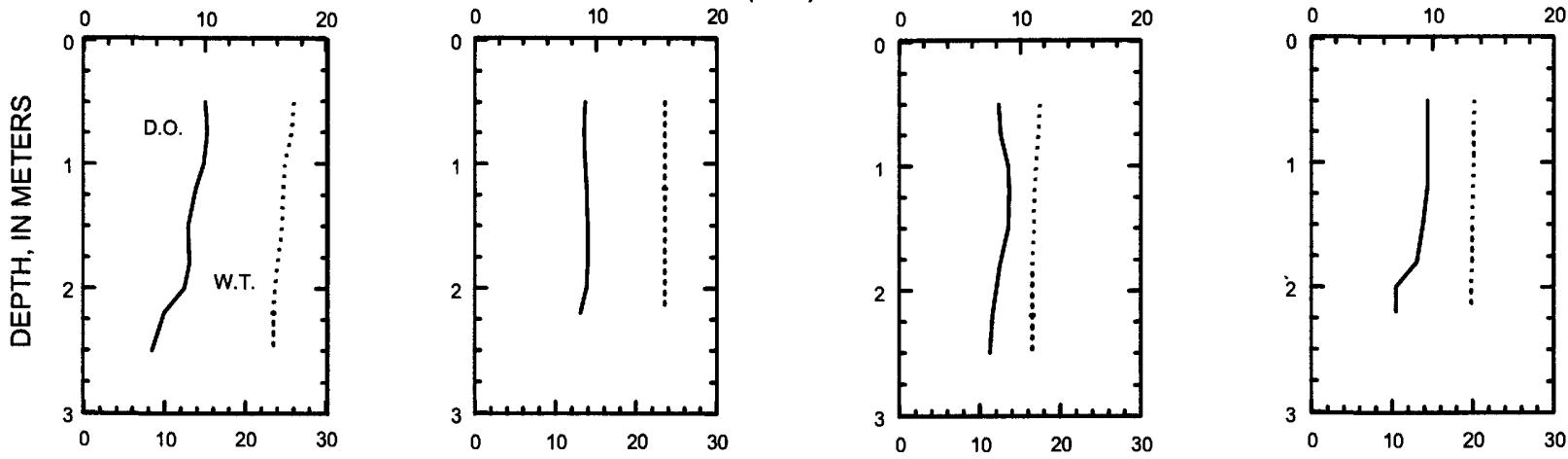

WATER TEMPERATURE (W.T.) IN DEGREES CELSIUS
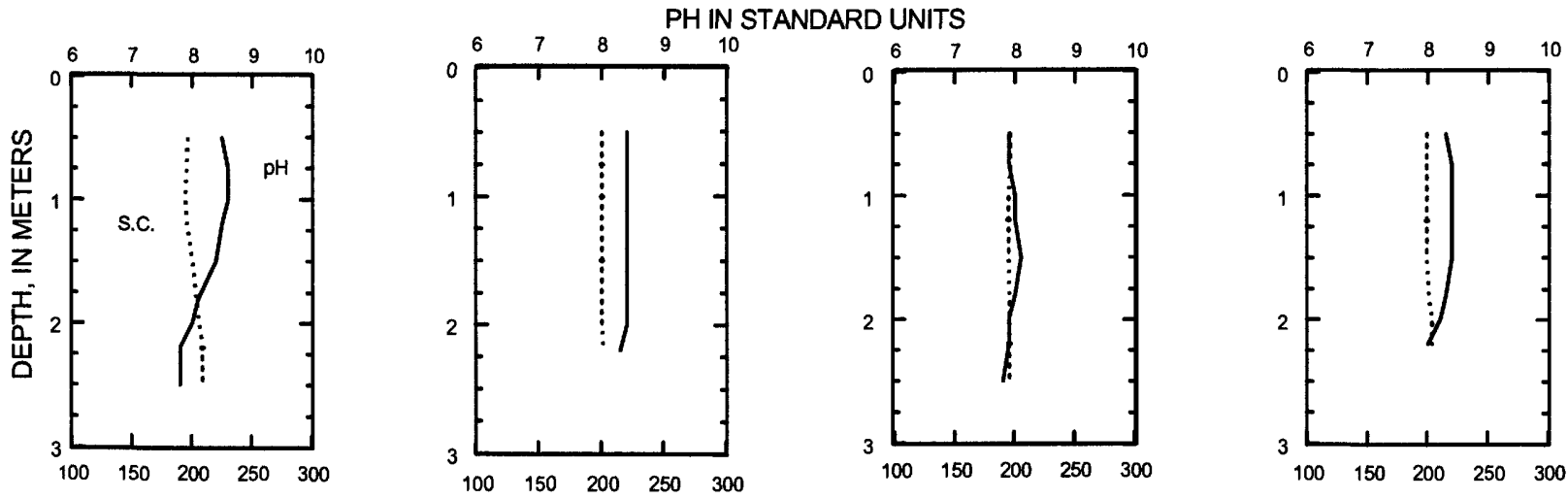

SPECIFIC CONDUCTANCE (S.C.) IN MICROSIEMENS PER CENTIMETER AT 25 DEGREES CELSIUS 

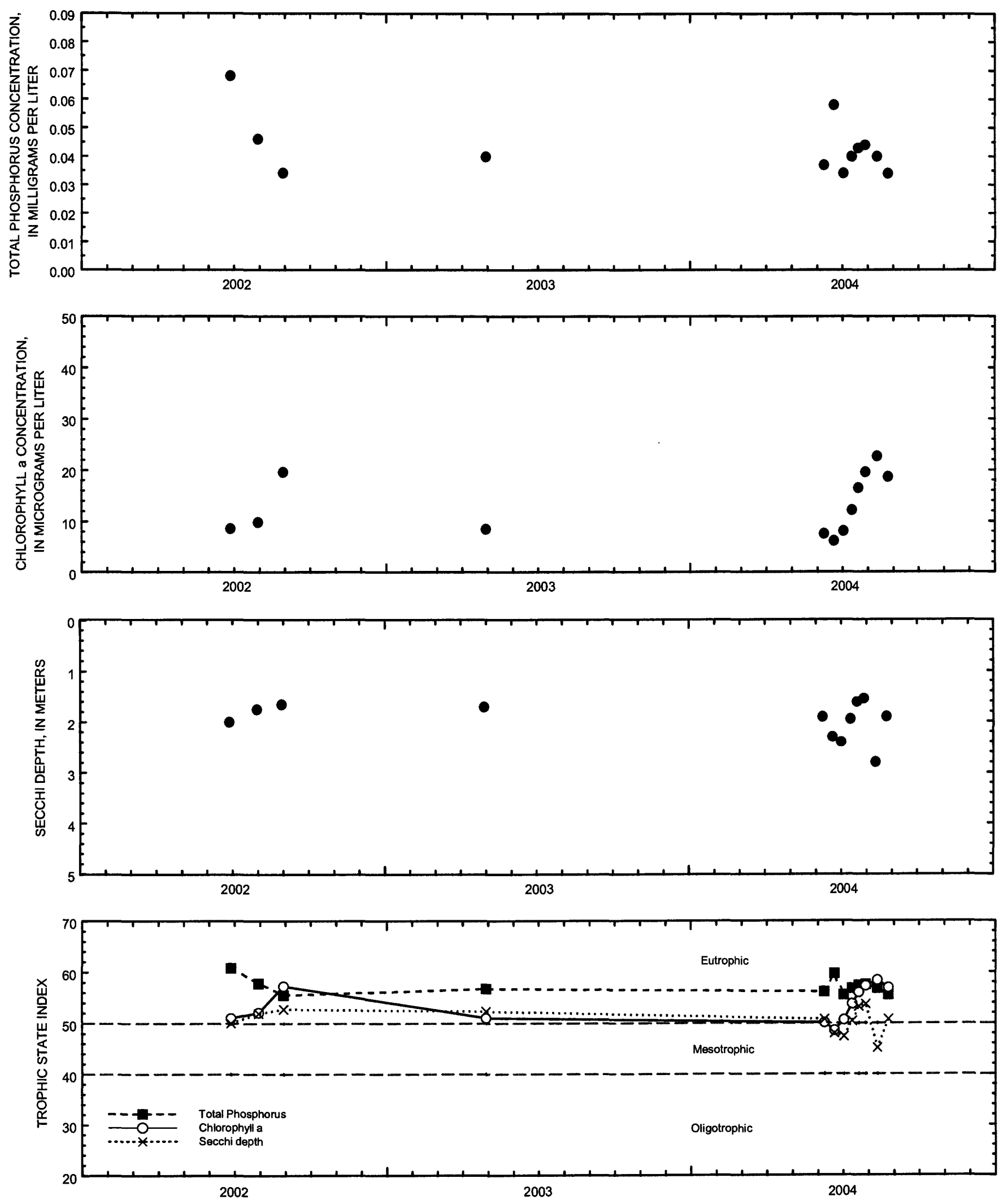

Surface total phosphorus, chlorophyll a concentrations, Secchi depths, and TSI data for Spooner Lake, Southeast Site, near Spooner, Wisconsin. 


\section{TICHIGAN LAKE NEAR WATERFORD, WI}

LOCATION.--Lat $42^{\circ} 48^{\prime} 54^{\prime \prime}$, long $88^{\circ} 12^{\prime} 33^{\prime \prime}$, in se $1 / 4$ SE $1 / 4$ sec.11, T.4 N., R.19 E., Racine County, Hydrologic Unit $07120006,3.5$ mi north of

Waterford.

PERIOD OF RECORD.--March 1994 to August 1996, April 2003 to current year.

REMARKS.--Lake sampled near center at the deep hole. Water-quality analyses done by Wisconsin State Laboratory of Hygiene.

WATER-QUALITY DATA, FEBRUARY 18 TO JUNE 7, 2004

(Milligrams per liter unless otherwise indicated)

Date

Lake stage (ft)

Secchi depth (m)

Depth of sample $(\mathrm{m})$

Chlorophyll a, phytoplankton ( $\mu \mathrm{g} / \mathrm{L})$

Water temperature $\left({ }^{\circ} \mathrm{C}\right)$

Specific conductance $(\mu \mathrm{S} / \mathrm{cm})$

$\mathrm{pH}$

Dissolved oxygen (mg/L)

Phosphorus, total (as P)
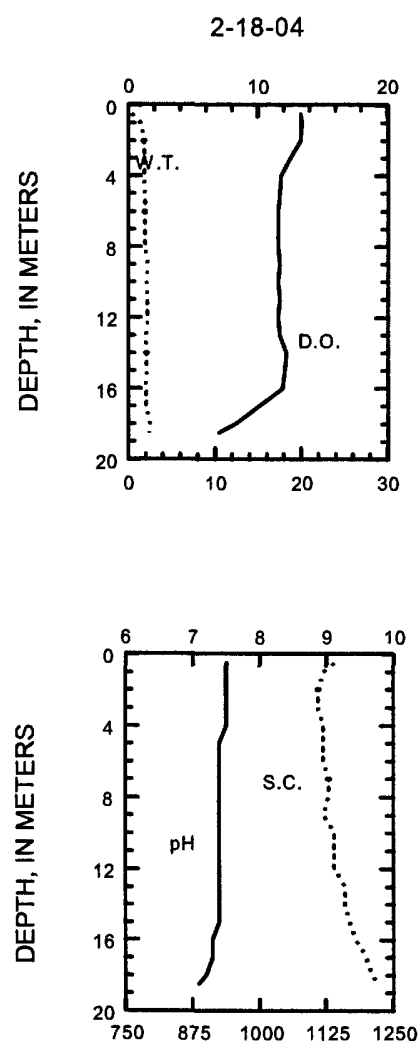

\begin{tabular}{cccccc} 
& $\frac{\text { Feb-18 }}{1.49}$ & & \multicolumn{3}{c}{$\frac{\text { Apr-12 }}{1.48}$} \\
& - & & \multicolumn{2}{c}{1.3} & \\
0.5 & & 18.5 & 0.5 & 18.5 \\
- & & - & 25.8 & - \\
0.4 & & 2.4 & 9.2 & 5.9 \\
1140 & & 1220 & 988 & 1010 \\
7.5 & & 7.1 & 8.5 & 7.7 \\
13.3 & & 7.0 & 12.9 & 5.6 \\
0.082 & & 0.121 & 0.067 & 0.137
\end{tabular}

4-12-04

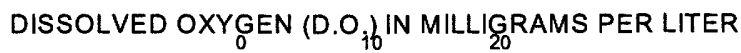

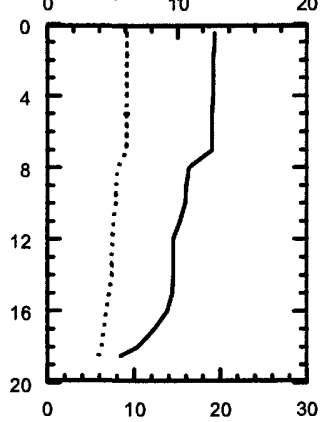

WATER TEMPERATURE (W.T.) IN DEGREES CELSIUS

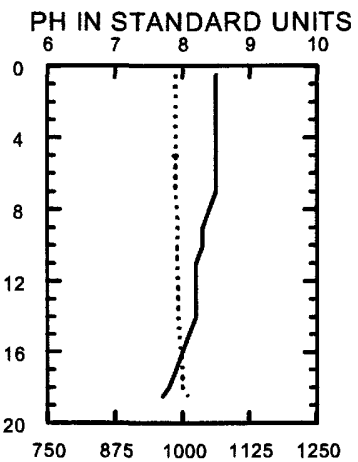

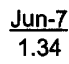

1.3

$\begin{array}{cc}0.5 & 17 \\ 19.9 & - \\ 22.8 & 9.9 \\ 846 & 1020 \\ 8.5 & 7.3 \\ 12.8 & 0.4 \\ 0.04 & 0.376\end{array}$

SPECIFIC CONDUCTANCE (S.C.) IN MICROSIEMENS PER CENTIMETER AT 25 DEGREES CELSIUS

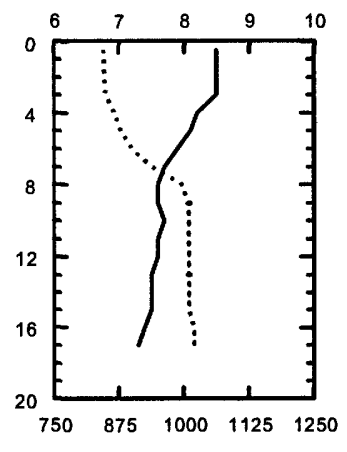


WATER-QUALITY DATA, JUNE 12 TO AUGUST 23, 2004

(Milligrams per liter unless otherwise indicated)

Date

Lake stage (ft)

Secchi depth $(\mathrm{m})$

Depth of sample $(\mathrm{m})$

Chlorophyll a, phytoplankton ( $\mu \mathrm{g} / \mathrm{L})$

Water temperature $\left({ }^{\circ} \mathrm{C}\right)$

Specific conductance $(\mu \mathrm{S} / \mathrm{cm})$

$\mathrm{pH}$

Dissolved oxygen (mg/L)

Phosphorus, total (as P)

$\begin{array}{ccc} & \frac{\text { Jun-12 }}{1.62} & \\ & 1.9 & \\ & & \\ 0.5 & & 18 \\ 11.6 & & - \\ 24.9 & & 9.9 \\ 800 & & 1050 \\ 8.4 & & 7.3 \\ 11.9 & & 0.2 \\ 0.037 & & 0.609\end{array}$

$\begin{array}{cccc} & & \frac{\text { Jul-16 }}{1.51} & \\ & & 1.1 & \\ 18.5 & 0.5 & & 18 \\ -- & 24.0 & - \\ 9.9 & 21.8 & & 10.2 \\ 1050 & 846 & & 1060 \\ 7.3 & 8.3 & 7.0 \\ 0.2 & 10.3 & 0.2 \\ 0.609 & 0.038 & & 0.804\end{array}$

8-16-04

DISSOLVED OXYGEN (D.O.) IN MILLIGRAMS PER LITER

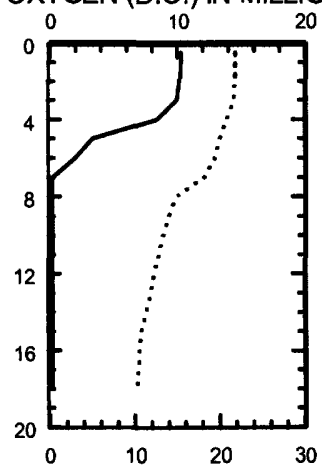

WATER TEMPERATURE (W.T.) IN DEGREES CELSIUS
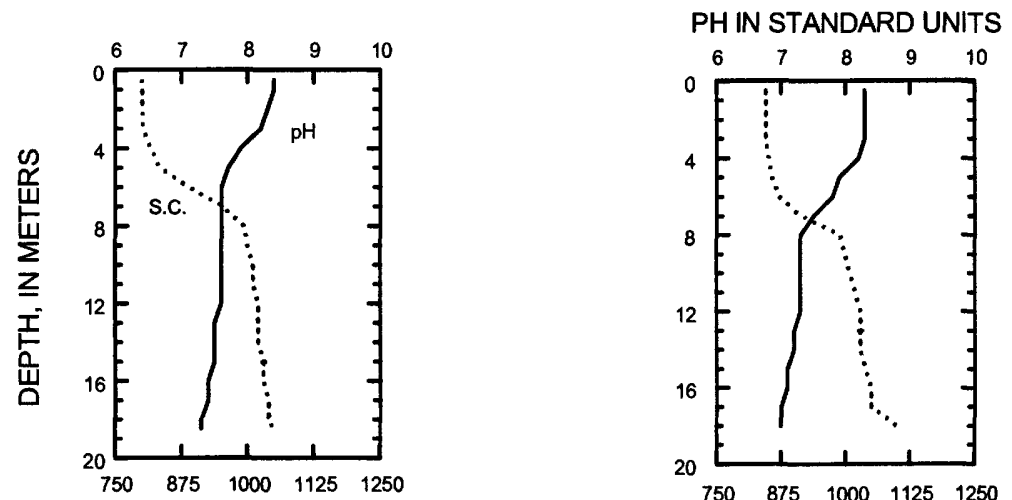
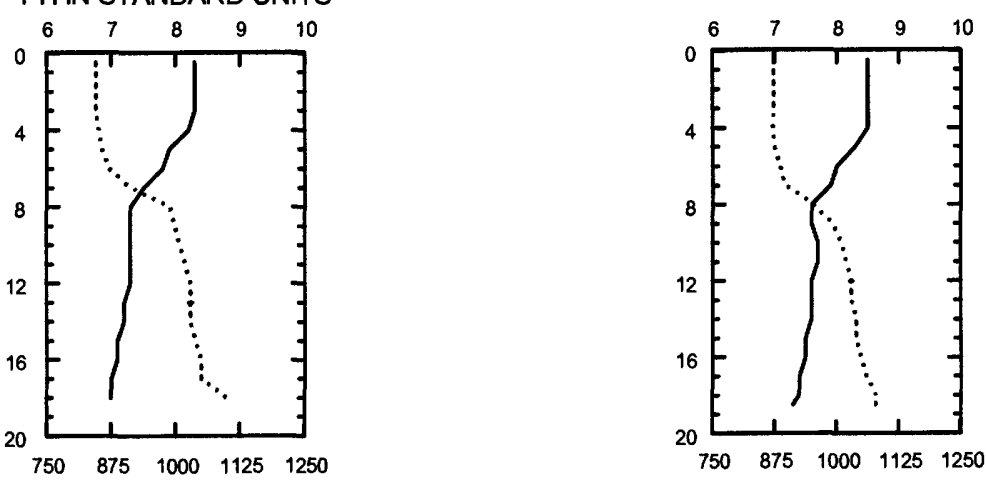

$9-23-04$

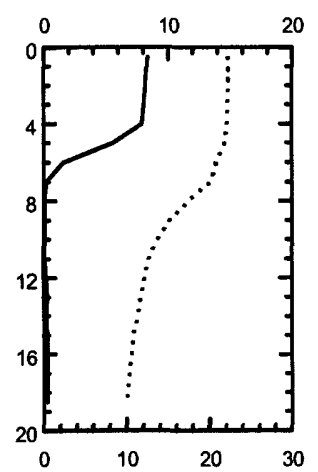

SPECIFIC CONDUCTANCE (S.C.) IN MICROSIEMENS PER CENTIMETER AT 25 DEGREES CELSIUS 

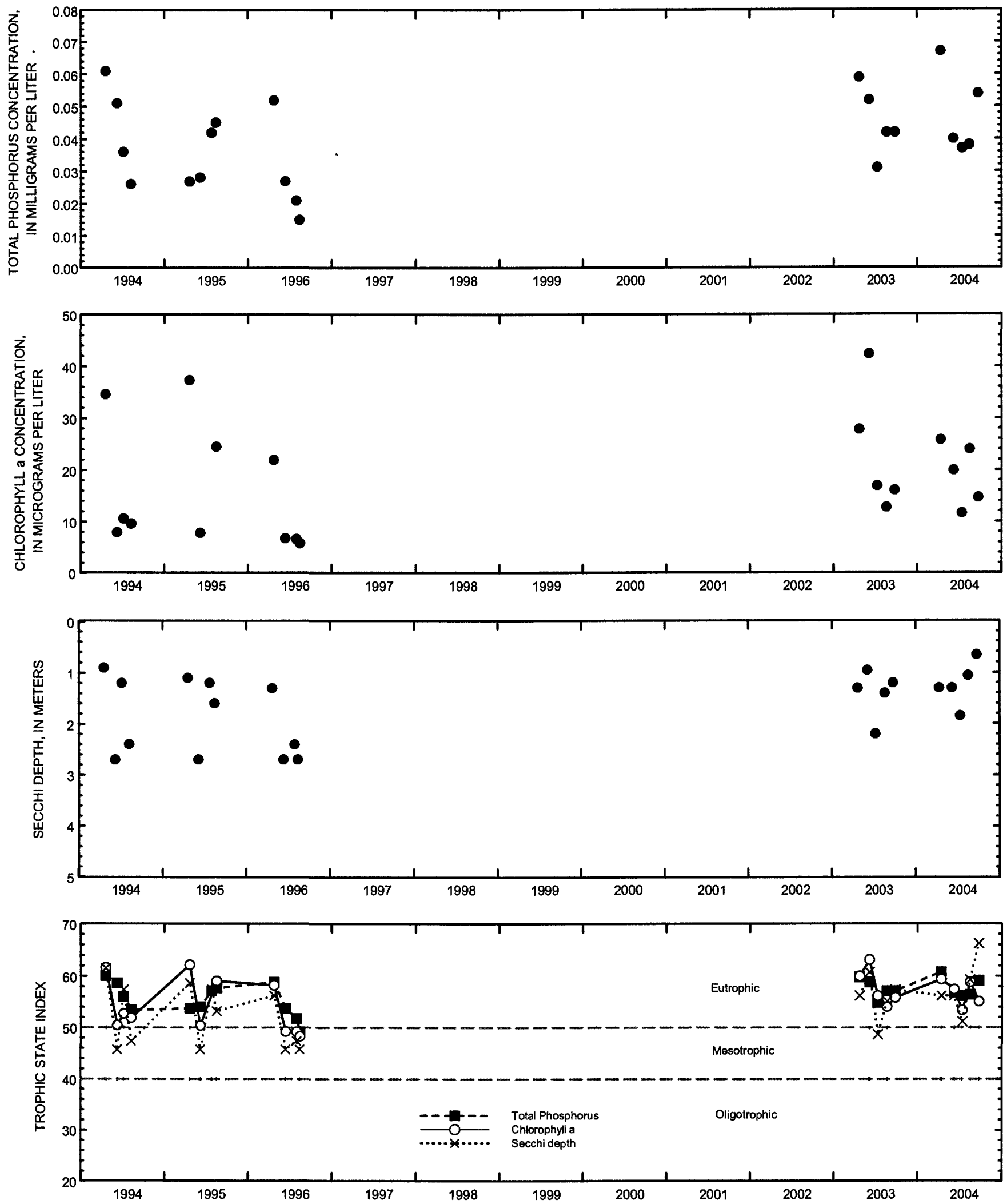

Surface total phosphorus, chlorophyll a concentrations, Secchi depths, and TSI data for Tichigan Lake, near Waterford, Wisconsin. 


\section{TWIN LAKES, EAST TWIN LAKE, NEAR WESTFIELD, WI}

LOCATION.--Lat 435' $30^{\prime \prime}$, long 89³5'07", in NE 1/4 NE 1/4 sec.3, T.17 N., R.8 E., Marquette County, Hydrologic Unit 04030201, near Westfield.

PERIOD OF RECORD.--June 2002 current year.

REMARKS.--Lake sampled at deepest hole. Water-quality analyses by Wisconsin State Laboratory of Hygiene.

WATER-QUALITY DATA, APRIL 20 TO AUGUST 23, 2004

(Milligrams per liter unless otherwise indicated)

Date

Lake stage (ft)

Secchi depth $(m)$

Depth of sample $(\mathrm{m})$

Chlorophyll a, phytoplankton $(\mu \mathrm{g} / \mathrm{L})$

Water temperature $\left({ }^{\circ} \mathrm{C}\right)$

Specific conductance $(\mu \mathrm{S} / \mathrm{cm})$

$\mathrm{pH}$

Dissolved oxygen (mg/L)

Phosphorus, total (as P)

\begin{tabular}{c} 
Apr-20 \\
\hline 9.02 \\
1.5 \\
0.5 \\
12.2 \\
14.1 \\
221 \\
8.3 \\
10.9 \\
0.034
\end{tabular}

$6-22-04$

7-8-04

DISSOLVED OXYGEN (D.O.) IN MILLIGRAMS PER LITER
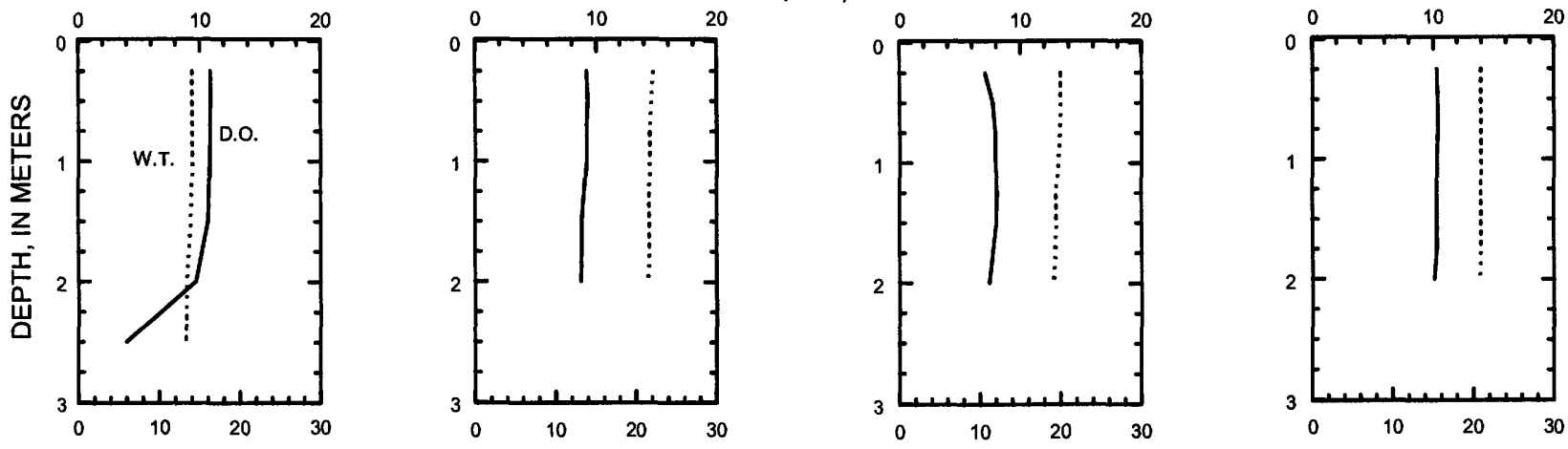

WATER TEMPERATURE (W.T.) IN DEGREES CELSIUS

PH IN STANDARD UNITS
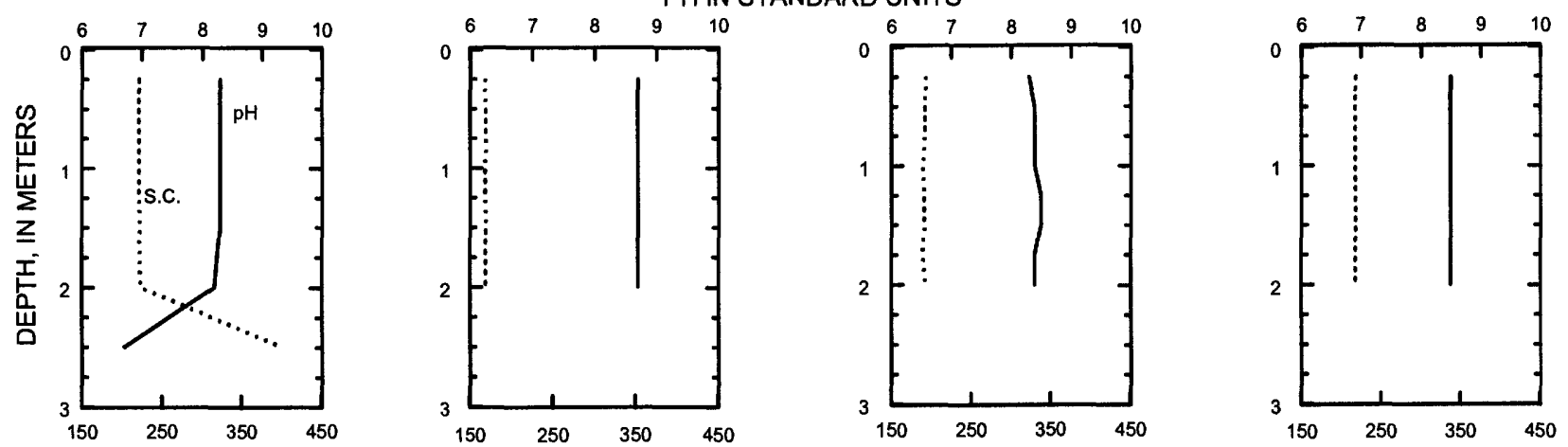

SPECIFIC CONDUCTANCE (S.C.) IN MICROSIEMENS PER CENTIMETER AT 25 DEGREES CELSIUS 

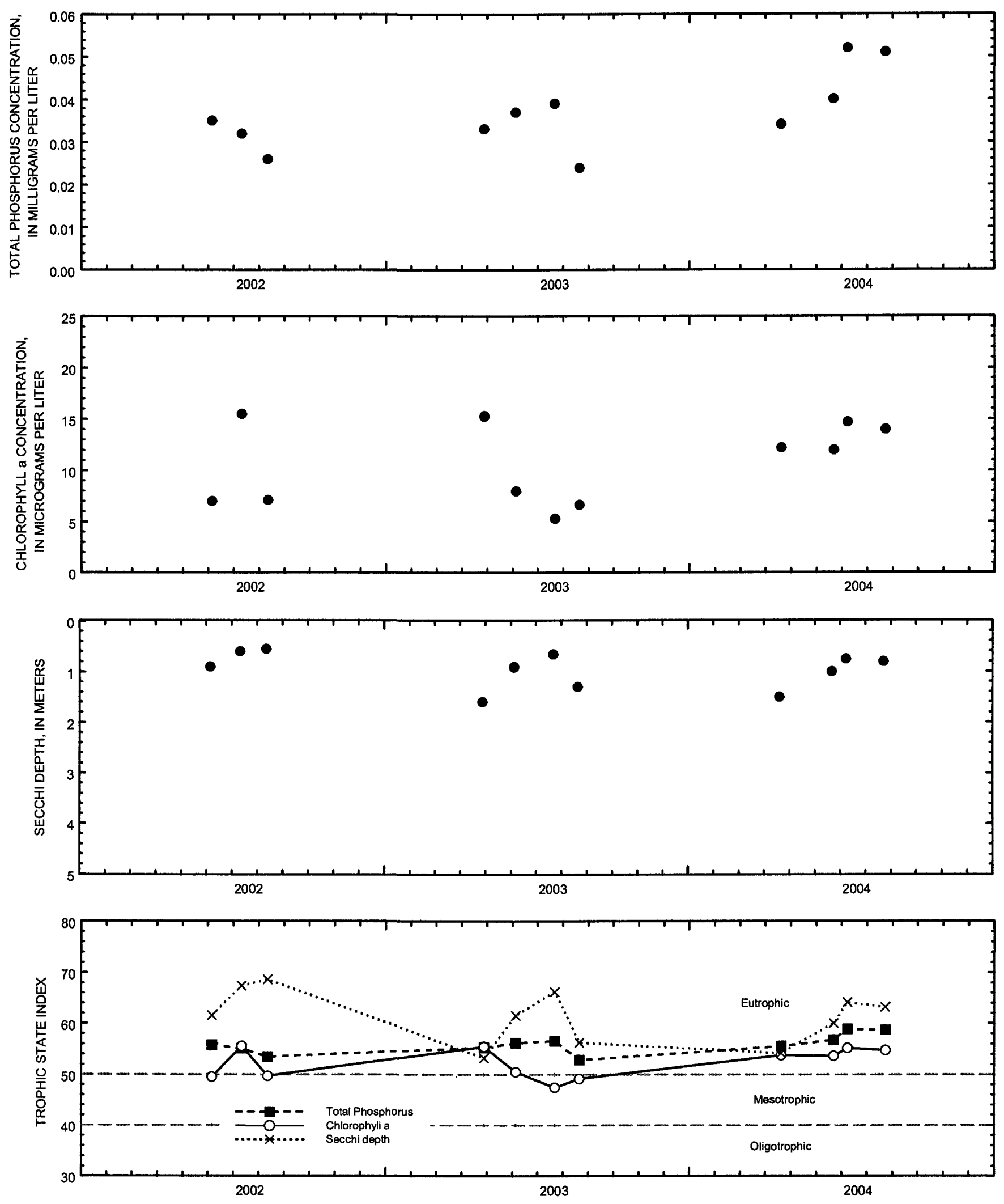

Surface total phosphorus, chlorophyll a concentrations, Secchi depths, and TSI data for Twin Lakes, East Twin Lake, near Westfield, Wisconsin. 


\section{TWIN LAKES, WEST TWIN LAKE, NEAR WESTFIELD, WI}

LOCATION.--Lat 4354'38", long 89³5'23", in SW 1/4 SE 1/4 sec.30, T.17 N., R.8 E., Marquette County, Hydrologic Unit 04030201, near Westfield.

PERIOD OF RECORD.--June 2002 to current year.

REMARKS.--Lake sampled at deepest hole. Water-quality analyses by Wisconsin State Laboratory of Hygiene.

WATER-QUALITY DATA, APRIL 20 TO AUGUST 23, 2004

(Milligrams per liter unless otherwise indicated)

Date

Lake stage (ft)

Secchi depth (m)

Depth of sample (m)

Chlorophyll a, phytoplankton ( $\mu \mathrm{g} / \mathrm{L}$ )

Water temperature $\left({ }^{\circ} \mathrm{C}\right)$

Specific conductance $(\mu \mathrm{S} / \mathrm{cm})$

$\mathrm{pH}$

Dissolved oxygen (mg/L)

Phosphorus, total (as P)

\begin{tabular}{cc}
\multicolumn{2}{c}{ Apr-2 } \\
\multicolumn{2}{c}{9.02} \\
\multicolumn{2}{c}{1.8} \\
0.5 & \\
8.4 & \\
13.4 \\
299 \\
8.2 \\
11.9 \\
0.040
\end{tabular}

$\frac{\text { Apr-20 }}{9.02}$

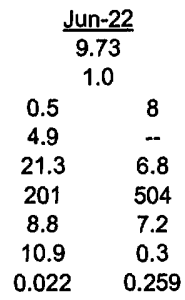

\begin{tabular}{|c|c|}
\hline \multicolumn{2}{|c|}{ Jul-8 } \\
\hline & \\
\hline \multicolumn{2}{|c|}{1.1} \\
\hline 0.5 & 8.5 \\
\hline 11.3 & -- \\
\hline 20.2 & 6.8 \\
\hline 211 & 564 \\
\hline 8.9 & 7.1 \\
\hline 11.2 & 0.2 \\
\hline 0.030 & 0.607 \\
\hline
\end{tabular}

$\begin{array}{ccc} & \text { Aug-23 } & \\ & 10.05 & \\ & 1.2 & \\ 0.5 & 2.8 & 8 \\ 8.0 & 9.4 & - \\ 20.6 & 19.7 & 7.5 \\ 239 & 241 & 609 \\ 8.8 & 8.8 & 7.1 \\ 12.8 & 13.2 & 0.3 \\ 0.018 & 0.019 & 0.102\end{array}$

4-20-04

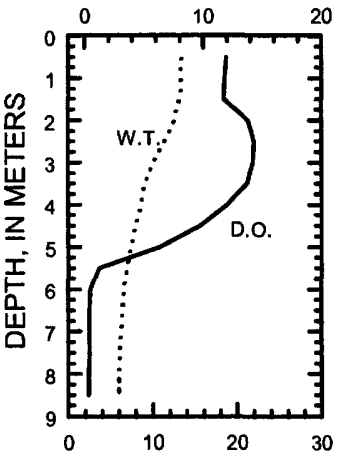

$6-22-04$

DISSOLVED OXYGEN (D.O.) IN MILLIGRAMS PER LITER

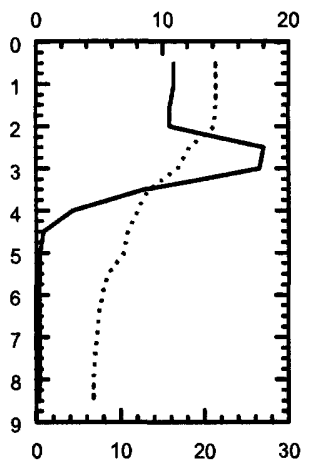

WATER TEMPERATURE (W.T.) IN DEGREES CELSIUS

PH IN STANDARD UNITS
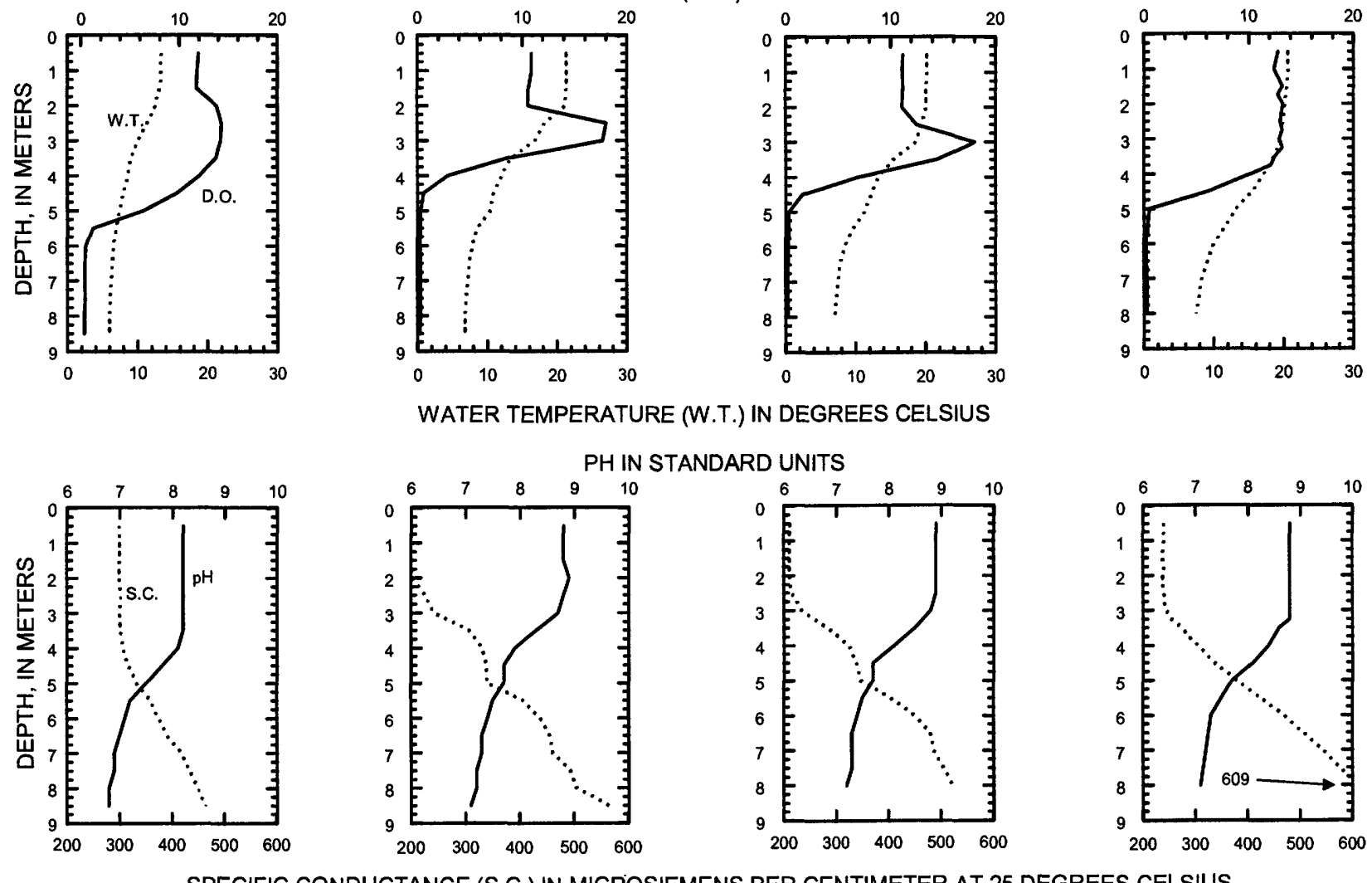

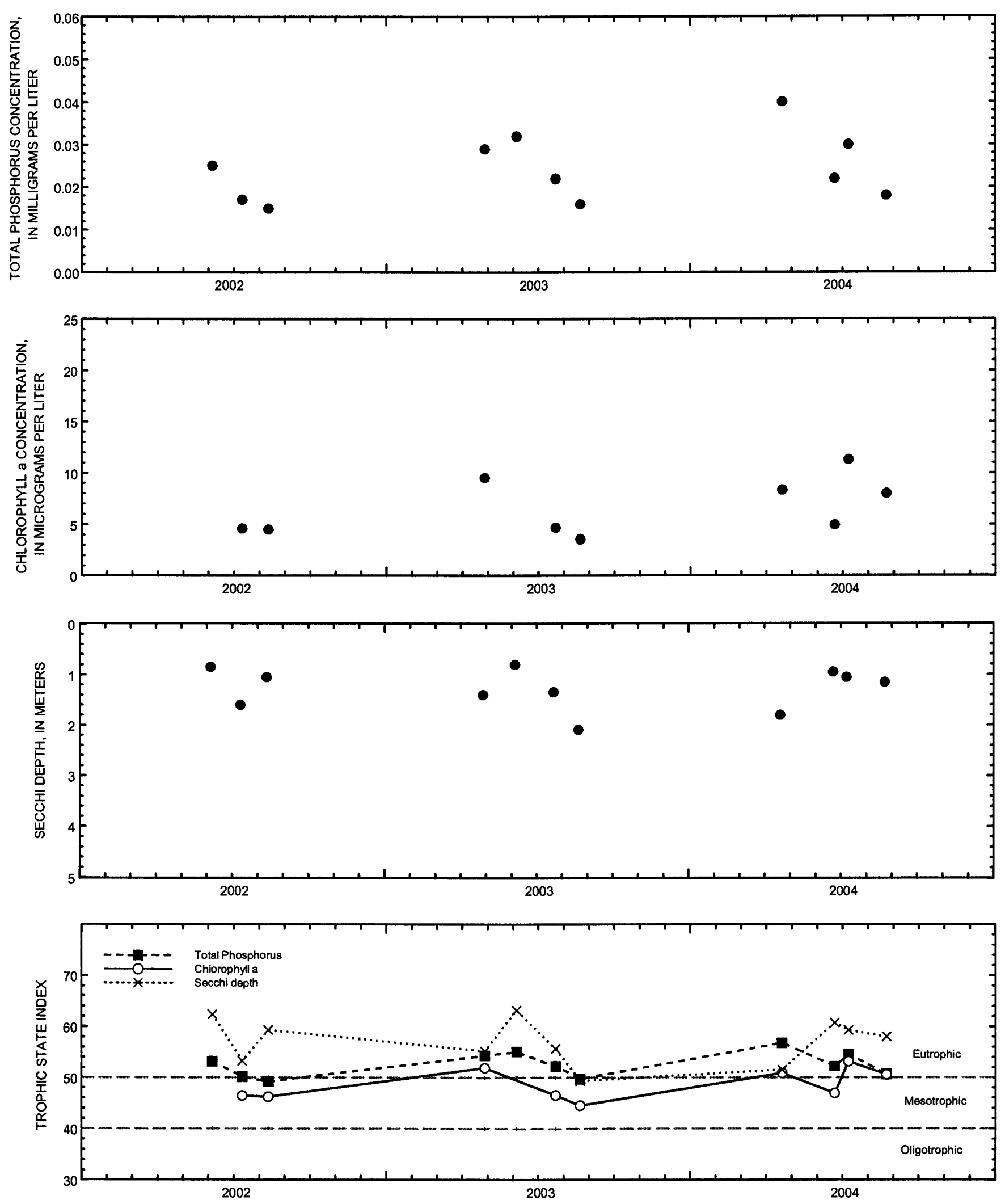

Surface total phosphorus, chlorophyll a concentrations, Secchi depths, and TSI data for Twin Lakes, West Twin Lake, near Westfield, Wisconsin. 


\section{WATERFORD WATERWAY, FOX RIVER SITE, NEAR WATERFORD, WI}

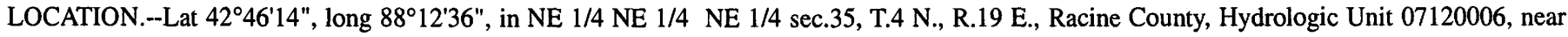
Waterford.

PERIOD OF RECORD.-April 2003 to current year.

REMARKS.--Lake sampled at Fox River site. River was ice-covered during February sampling. Water-quality analyses done by Wisconsin State Laboratory of Hygiene.

\section{WATER-QUALITY DATA, FEBRUARY 18 TO JUNE 7, 2004}

(Milligrams per liter unless otherwise indicated)

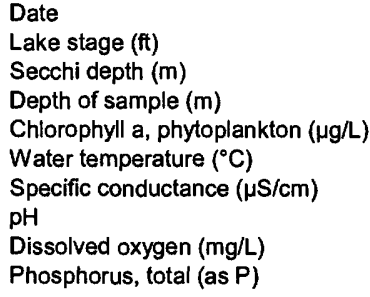

2-18-04
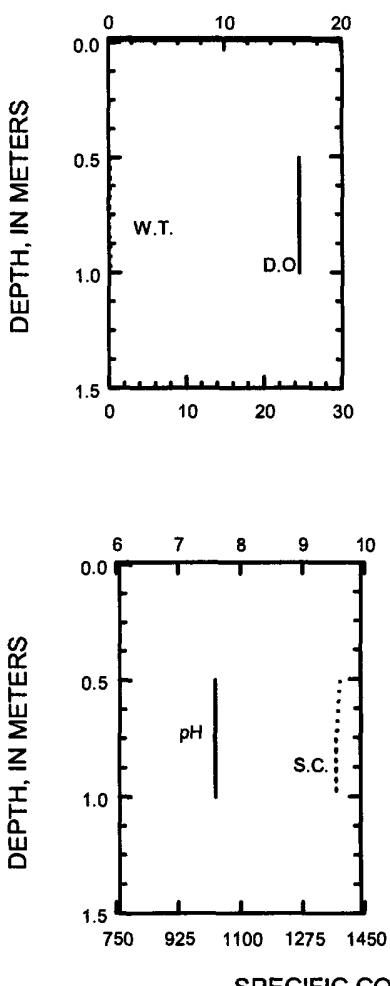

4-12-04

DISSOLVED OXYGEN (D.O.) IN MILLIGRAMS PER LITER

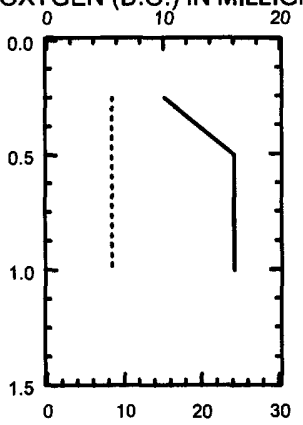

WATER TEMPERATURE (W.T.) IN DEGREES CELSIUS

PH IN STANDARD UNITS

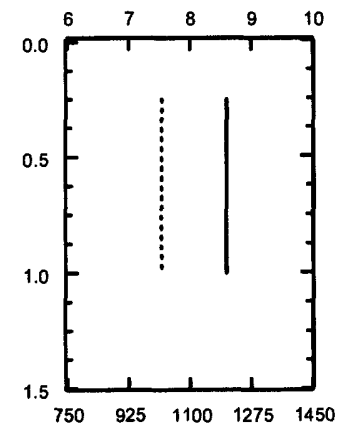

\begin{tabular}{c} 
Apr-12 \\
\hline 1.48 \\
0.5 \\
0.5 \\
60.1 \\
8.4 \\
1020 \\
8.6 \\
16.1 \\
0.076
\end{tabular}

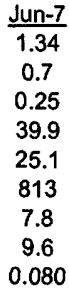

$6-7-04$
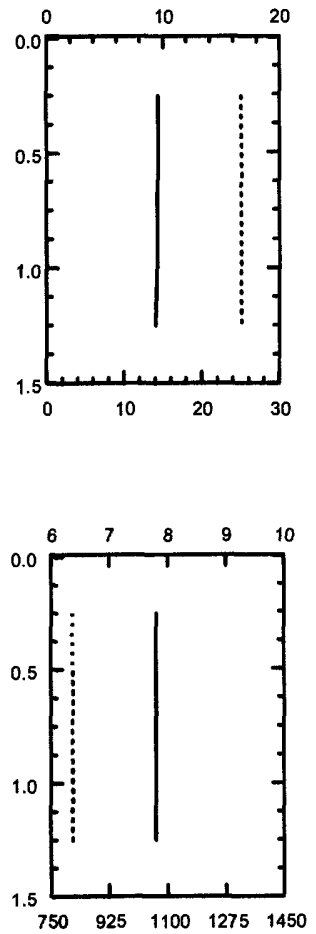

SPECIFIC CONDUCTANCE (S.C.) IN MICROSIEMENS PER CENTIMETER AT 25 DEGREES CELSIUS 
WATER-QUALITY DATA, JULY 12 TO SEPTEMBER 23, 2004

(Milligrams per liter unless otherwise indicated)

Date

Lake stage (ft)

Secchi depth $(\mathrm{m})$

Depth of sample $(\mathrm{m})$

Chlorophyll a, phytoplankton $(\mu \mathrm{g} / \mathrm{L})$

Water temperature $\left({ }^{\circ} \mathrm{C}\right)$

Specific conductance $(\mu S / \mathrm{cm})$

$\mathrm{pH}$

Dissolved oxygen (mg/L)

Phosphorus, total (as P)

$7-12-04$
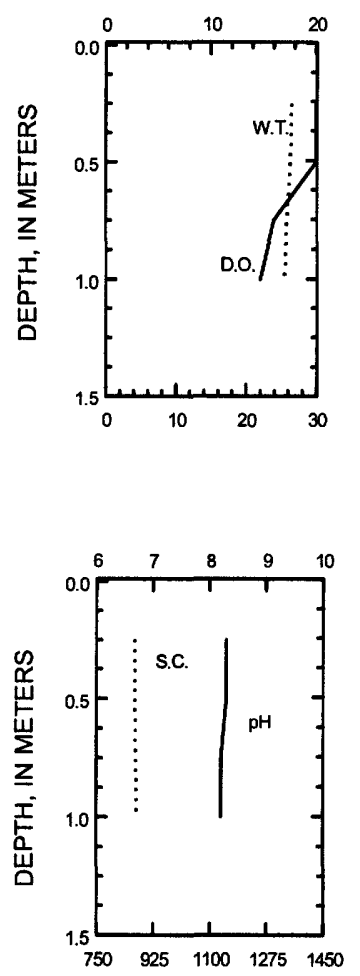

8-16-04

DISSOLVED OXYGEN (D.O.) IN MILLIGRAMS PER LITER

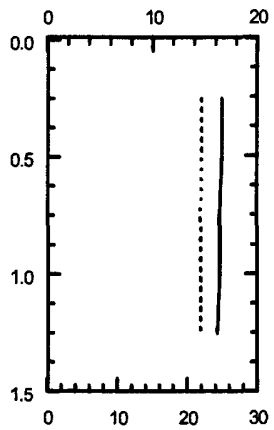

WATER TEMPERATURE (W.T.) IN DEGREES CELSIUS

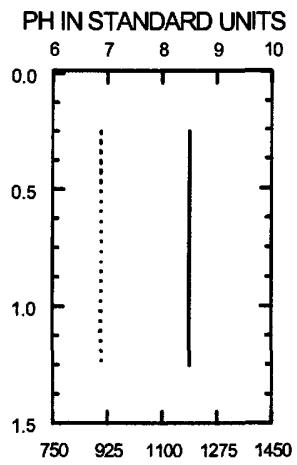

\begin{tabular}{c} 
Aug-16 \\
\hline 1.51 \\
0.2 \\
0.5 \\
149.0 \\
22.0 \\
904 \\
8.5 \\
16.6 \\
0.166
\end{tabular}

Sep-23

1.30

0.25

97.1

22.2

995

8.8

$9-23-04$
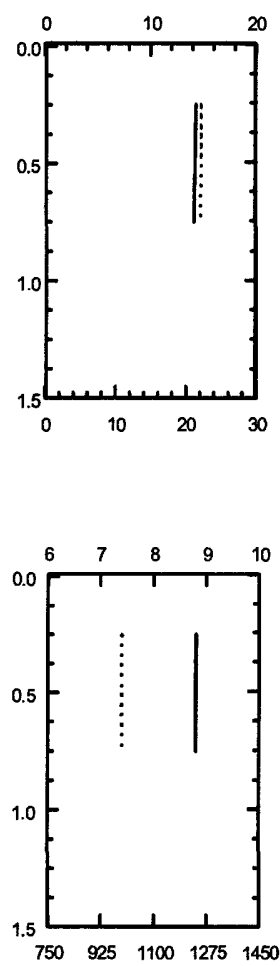

SPECIFIC CONDUCTANCE (S.C.) IN MICROSIEMENS PER CENTIMETER AT 25 DEGREES CEISIUS 

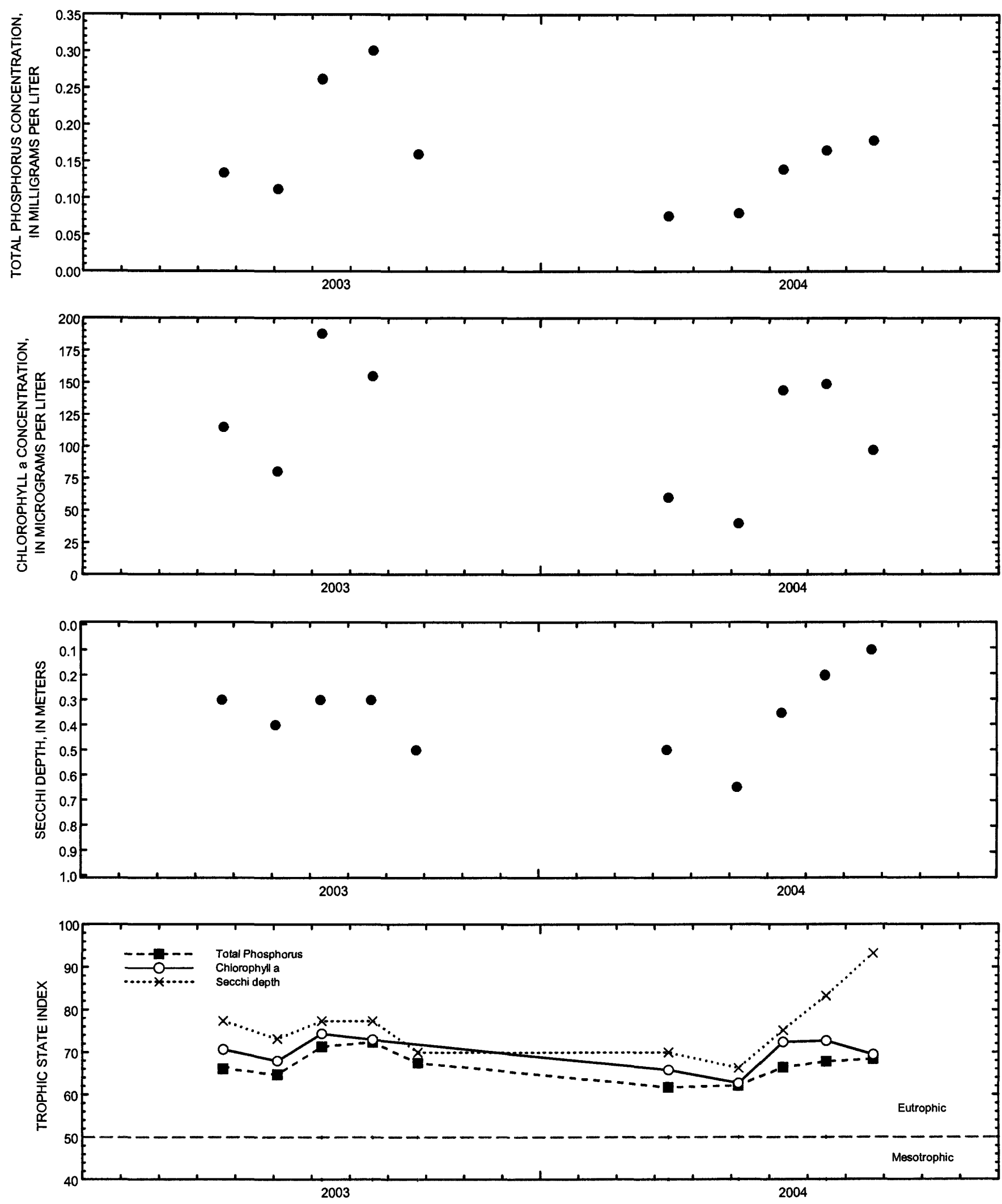

Surface total phosphorus, chlorophyll a concentrations, Secchi depths, and

TSI data for Waterford Waterway, Fox River Site, near Waterford, Wisconsin. 


\section{WAZEE LAKE AT DEEP HOLE NEAR BLACK RIVER FALLS, WI}

LOCATION.--Lat 4417'30", long 9043'29", in SE 1/4 SE 1/4 sec.15, T.21 N., R.3 W., Jackson County, Hydrologic Unit $07040007,7.0$ mi east of Black River Falls.

PERIOD OF RECORD.--November 1999 to current year.

REMARKS.--Date published under station number 441721090431700 in water year 2000 . Water-quality analyses done by Wisconsin State Laboratory of Hygiene.

WATER-QUALITY DATA, DECEMBER 6, 2003

(Milligrams per liter unless otherwise indicated)

Date

Lake stage ( $\mathrm{ft}$ )

Secchi depth $(m)$

Depth of sample $(\mathrm{m})$

Chlorophyll a, phytoplankton $(\mu \mathrm{g} / \mathrm{L})$

Water temperature $\left({ }^{\circ} \mathrm{C}\right)$

Specific conductance $(\mu \mathrm{S} / \mathrm{cm})$

$\mathrm{pH}$

Dissolved oxygen (mg/L)

Phosphorus, total (as P)

$\begin{array}{ccc} & \frac{\text { Dec-6 }}{3.42} & \\ & 12.8 & \\ 0.5 & & 34 \\ 6.79 & & - \\ 5.4 & & 5.1 \\ 246 & & 248 \\ 8.0 & & 7.6 \\ 12.7 & & 11.7 \\ 0.006 & & 0.002\end{array}$

12-6-03

DISSOLVED OXYGEN (D.O.) IN MILLIGRAMS PER LITER

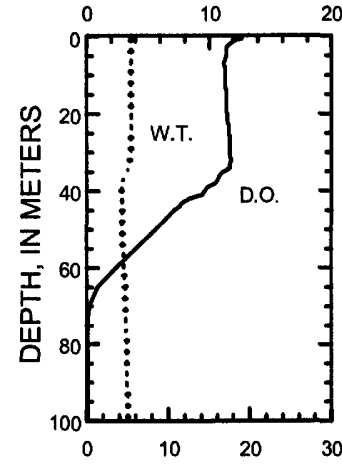

WATER TEMPERATURE (W.T.) IN DEGREES CELCIUS

PH IN STANDARD UNITS

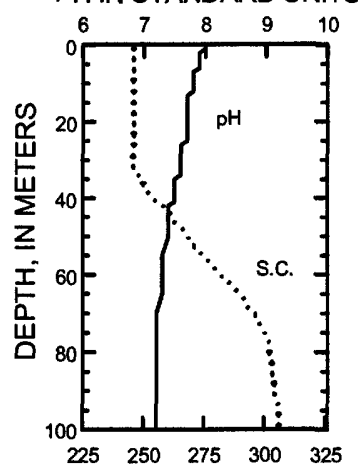

SPECIFIC CONDUCTANCE (S.C.) IN MICROSIEMENS PER CENTIMETER AT 25 DEGREES CELCIUS 

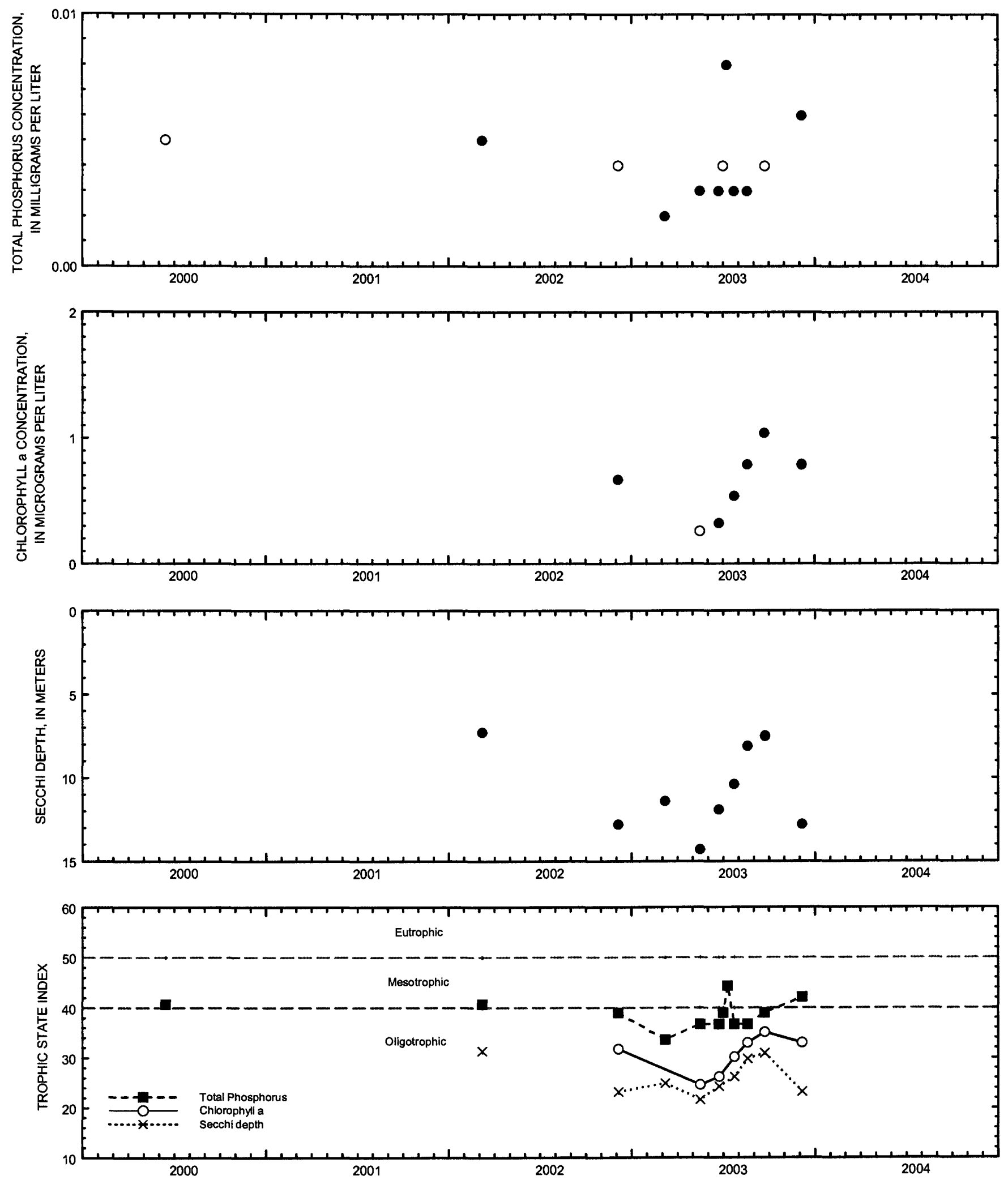

Surface total phosphorus, chlorophyll a concentrations, Secchi depths, and TSI data for Wazee Lake, Deep Hole, near Black River Falls, Wisconsin.

(Circles on the first two plots indicate iaboratory detection limit for selected anaiyses. Actual concentrations for these particuiar analyses are less than the plotted circles.) 


\section{WIND LAKE AT OUTLET AT WIND LAKE, WI}

LOCATION.--Lat $42^{\circ} 48^{\prime} 48^{\prime \prime}$ long $88^{\circ} 08^{\prime} 31^{\prime \prime}$, in NE $1 / 4$ NW 1/4 sec.16, T.4 N., R.20 E., Racine County, Hydrologic Unit 07120006, at Wind Lake.

DRAINAGE AREA.--39.6 $\mathrm{mi}^{2}$.

PERIOD OF RECORD.--March 1985 to current year. Prior to October 2000, published as "Wind Lake Outlet".

REVISED RECORDS.--WDR WI-91-1: 1988(m).

GAGE.--Water-stage recorder and concrete dam. Datum of gage is $760.30 \mathrm{ft}$ above sea level. Prior to Oct. 2, 1987, nonrecording gage at same site and datum.

REMARKS.--Lake level regulated by dam with two 10-foot gates at outlet. Lake ice-covered Dec. 1 to Mar. 11. Prior to October 1987, published as Wind Lake at Wind Lake, Wis. Gage-height telemeter at station.

EXTREMES FOR PERIOD OF RECORD.--Maximum gage height, $8.93 \mathrm{ft}$, June 15, 1999; minimum recorded, $5.95 \mathrm{ft}$, Jan. 2 , 1996.

EXTREMES FOR CURRENT YEAR.--Maximum recorded gage height, $8.57 \mathrm{ft}$, June 7; minimum recorded, $7.43 \mathrm{ft}$, Oct. 28.

GAGE HEIGHT, FEET

WATER YEAR OCTOBER 2003 TO SEPTEMBER 2004 DAILY MEAN VALUES

\begin{tabular}{|c|c|c|c|c|c|c|c|c|c|c|c|c|}
\hline DAY & OCT & NOV & DEC & JAN & FEB & MAR & APR & MAY & JUN & JUL & AUG & SEP \\
\hline $\begin{array}{l}1 \\
2 \\
3 \\
4 \\
5\end{array}$ & $\begin{array}{l}7.55 \\
7.53 \\
7.52 \\
7.53 \\
7.51\end{array}$ & $\begin{array}{l}7.47 \\
7.54 \\
7.63 \\
7.69 \\
7.71\end{array}$ & $\begin{array}{l}7.84 \\
7.84 \\
7.84 \\
7.85 \\
7.88\end{array}$ & $\begin{array}{l}8.15 \\
8.16 \\
8.17 \\
8.16 \\
8.16\end{array}$ & $\begin{array}{l}8.14 \\
8.13 \\
8.14 \\
8.13 \\
8.12\end{array}$ & $\begin{array}{l}8.18 \\
8.18 \\
8.15 \\
8.12 \\
8.26\end{array}$ & $\begin{array}{l}8.14 \\
8.11 \\
8.13 \\
8.13 \\
8.11\end{array}$ & $\begin{array}{l}8.23 \\
8.20 \\
8.16 \\
8.12 \\
8.13\end{array}$ & $\begin{array}{l}8.18 \\
8.12 \\
8.10 \\
8.14 \\
8.33\end{array}$ & $\begin{array}{l}8.11 \\
8.11 \\
8.13 \\
8.20 \\
8.23\end{array}$ & $\begin{array}{l}8.18 \\
8.17 \\
8.17 \\
8.23 \\
8.20\end{array}$ & $\begin{array}{l}8.07 \\
8.06 \\
8.06 \\
8.05 \\
8.04\end{array}$ \\
\hline $\begin{array}{r}6 \\
7 \\
8 \\
9 \\
10\end{array}$ & $\begin{array}{l}7.51 \\
7.50 \\
7.50 \\
7.50 \\
7.50\end{array}$ & $\begin{array}{l}7.71 \\
7.70 \\
7.68 \\
7.67 \\
7.67\end{array}$ & $\begin{array}{l}7.89 \\
7.90 \\
7.92 \\
7.95 \\
8.13\end{array}$ & $\begin{array}{l}8.13 \\
8.11 \\
8.08 \\
8.07 \\
8.06\end{array}$ & $\begin{array}{l}8.13 \\
8.12 \\
8.11 \\
8.10 \\
8.08\end{array}$ & $\begin{array}{l}8.32 \\
8.31 \\
8.24 \\
8.16 \\
8.08\end{array}$ & $\begin{array}{l}8.11 \\
8.13 \\
8.21 \\
8.22 \\
8.20\end{array}$ & $\begin{array}{l}8.14 \\
8.15 \\
8.14 \\
8.22 \\
8.20\end{array}$ & $\begin{array}{l}8.49 \\
8.52 \\
8.35 \\
8.15 \\
8.15\end{array}$ & $\begin{array}{l}8.25 \\
8.30 \\
8.32 \\
8.35 \\
8.27\end{array}$ & $\begin{array}{l}8.17 \\
8.15 \\
8.14 \\
8.13 \\
8.11\end{array}$ & $\begin{array}{l}8.03 \\
8.02 \\
8.01 \\
7.99 \\
7.97\end{array}$ \\
\hline $\begin{array}{l}11 \\
12 \\
13 \\
14 \\
15\end{array}$ & $\begin{array}{l}7.49 \\
7.50 \\
7.49 \\
7.53 \\
7.52\end{array}$ & $\begin{array}{l}7.67 \\
7.67 \\
7.66 \\
7.64 \\
7.64\end{array}$ & $\begin{array}{l}8.17 \\
8.20 \\
8.25 \\
8.30 \\
8.33\end{array}$ & $\begin{array}{l}8.06 \\
8.07 \\
8.09 \\
8.10 \\
8.12\end{array}$ & $\begin{array}{l}8.08 \\
8.08 \\
8.07 \\
8.07 \\
8.06\end{array}$ & $\begin{array}{l}8.03 \\
8.01 \\
8.07 \\
8.15 \\
8.23\end{array}$ & $\begin{array}{l}8.17 \\
8.13 \\
8.08 \\
8.05 \\
8.08\end{array}$ & $\begin{array}{l}8.19 \\
8.16 \\
8.24 \\
8.24 \\
8.12\end{array}$ & $\begin{array}{l}8.22 \\
8.26 \\
8.29 \\
8.31 \\
8.28\end{array}$ & $\begin{array}{l}8.23 \\
8.25 \\
8.25 \\
8.22 \\
8.21\end{array}$ & $\begin{array}{l}8.09 \\
8.07 \\
8.06 \\
8.04 \\
8.03\end{array}$ & $\begin{array}{l}7.95 \\
7.94 \\
7.93 \\
7.91 \\
7.90\end{array}$ \\
\hline $\begin{array}{l}16 \\
17 \\
18 \\
19 \\
20\end{array}$ & $\begin{array}{l}7.51 \\
7.49 \\
7.48 \\
7.48 \\
7.47\end{array}$ & $\begin{array}{l}7.64 \\
7.64 \\
7.73 \\
7.75 \\
7.74\end{array}$ & $\begin{array}{l}8.28 \\
8.21 \\
8.20 \\
8.17 \\
8.14\end{array}$ & $\begin{array}{l}8.13 \\
8.14 \\
8.15 \\
8.15 \\
8.15\end{array}$ & $\begin{array}{l}8.05 \\
8.04 \\
8.04 \\
8.04 \\
8.05\end{array}$ & $\begin{array}{l}8.22 \\
8.11 \\
8.07 \\
8.10 \\
8.17\end{array}$ & $\begin{array}{l}8.15 \\
8.22 \\
8.22 \\
8.23 \\
8.19\end{array}$ & $\begin{array}{l}8.04 \\
8.10 \\
8.32 \\
8.31 \\
8.15\end{array}$ & $\begin{array}{l}8.22 \\
8.29 \\
8.27 \\
8.18 \\
8.16\end{array}$ & $\begin{array}{l}8.19 \\
8.20 \\
8.18 \\
8.18 \\
8.18\end{array}$ & $\begin{array}{l}8.01 \\
8.05 \\
8.05 \\
8.04 \\
8.03\end{array}$ & $\begin{array}{l}7.91 \\
7.89 \\
7.87 \\
7.85 \\
7.84\end{array}$ \\
\hline $\begin{array}{l}21 \\
22 \\
23 \\
24 \\
25\end{array}$ & $\begin{array}{l}7.48 \\
7.47 \\
7.46 \\
7.46 \\
7.50\end{array}$ & $\begin{array}{l}7.76 \\
7.77 \\
7.79 \\
7.78 \\
7.78\end{array}$ & $\begin{array}{l}8.13 \\
8.16 \\
8.20 \\
8.21 \\
8.19\end{array}$ & $\begin{array}{l}8.15 \\
8.15 \\
8.16 \\
8.18 \\
8.19\end{array}$ & $\begin{array}{l}8.07 \\
8.06 \\
8.08 \\
8.08 \\
8.09\end{array}$ & $\begin{array}{l}8.21 \\
8.19 \\
8.12 \\
8.05 \\
8.00\end{array}$ & $\begin{array}{l}8.21 \\
8.18 \\
8.16 \\
8.17 \\
8.20\end{array}$ & $\begin{array}{l}8.10 \\
8.27 \\
8.42 \\
8.52 \\
8.53\end{array}$ & $\begin{array}{l}8.26 \\
8.33 \\
8.29 \\
8.28 \\
8.25\end{array}$ & $\begin{array}{l}8.20 \\
8.25 \\
8.25 \\
8.23 \\
8.22\end{array}$ & $\begin{array}{l}8.01 \\
7.99 \\
7.99 \\
7.99 \\
8.04\end{array}$ & $\begin{array}{l}7.82 \\
7.81 \\
7.80 \\
7.78 \\
7.77\end{array}$ \\
\hline $\begin{array}{l}26 \\
27 \\
28 \\
29 \\
30 \\
31\end{array}$ & $\begin{array}{l}7.49 \\
7.48 \\
7.47 \\
7.47 \\
7.47 \\
7.47\end{array}$ & $\begin{array}{r}7.79 \\
7.80 \\
7.83 \\
7.82 \\
7.83 \\
--.\end{array}$ & $\begin{array}{l}8.17 \\
8.14 \\
8.14 \\
8.15 \\
8.15 \\
8.15\end{array}$ & $\begin{array}{l}8.20 \\
8.23 \\
8.22 \\
8.20 \\
8.18 \\
8.16\end{array}$ & $\begin{array}{r}8.10 \\
8.11 \\
8.13 \\
8.14 \\
\ldots . . \\
\ldots\end{array}$ & $\begin{array}{l}8.06 \\
8.09 \\
8.14 \\
8.23 \\
8.21 \\
8.20\end{array}$ & $\begin{array}{r}8.22 \\
8.23 \\
8.23 \\
8.22 \\
8.23 \\
---\end{array}$ & $\begin{array}{l}8.48 \\
8.39 \\
8.26 \\
8.18 \\
8.19 \\
8.23\end{array}$ & $\begin{array}{r}8.33 \\
8.40 \\
8.43 \\
8.34 \\
8.19 \\
---\end{array}$ & $\begin{array}{l}8.24 \\
8.25 \\
8.25 \\
8.23 \\
8.21 \\
8.20\end{array}$ & $\begin{array}{l}8.04 \\
8.05 \\
8.10 \\
8.11 \\
8.09 \\
8.08\end{array}$ & $\begin{array}{r}7.76 \\
7.75 \\
7.74 \\
7.71 \\
7.69 \\
\ldots\end{array}$ \\
\hline $\begin{array}{r}\text { MEAN } \\
\text { MAX } \\
\text { MIN }\end{array}$ & $\begin{array}{l}7.49 \\
7.55 \\
7.46\end{array}$ & $\begin{array}{l}7.71 \\
7.83 \\
7.47\end{array}$ & $\begin{array}{l}8.10 \\
8.33 \\
7.84\end{array}$ & $\begin{array}{l}8.14 \\
8.23 \\
8.06\end{array}$ & $\begin{array}{l}8.09 \\
8.14 \\
8.04\end{array}$ & $\begin{array}{l}8.15 \\
8.32 \\
8.00\end{array}$ & $\begin{array}{l}8.17 \\
8.23 \\
8.05\end{array}$ & $\begin{array}{l}8.23 \\
8.53 \\
8.04\end{array}$ & $\begin{array}{l}8.27 \\
8.52 \\
8.10\end{array}$ & $\begin{array}{l}8.22 \\
8.35 \\
8.11\end{array}$ & $\begin{array}{l}8.08 \\
8.23 \\
7.99\end{array}$ & $\begin{array}{l}7.90 \\
8.07 \\
7.69\end{array}$ \\
\hline
\end{tabular}

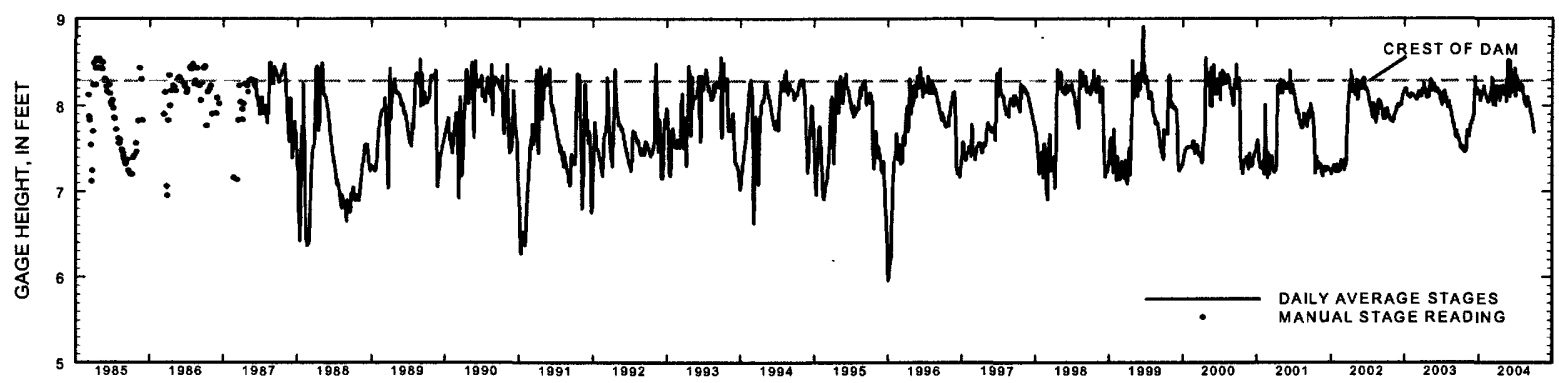




\section{WIND LAKE AT WIND LAKE, WI}

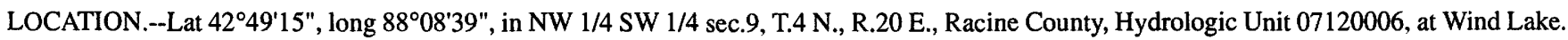
PERIOD OF RECORD.--February 1985 to current year.

REMARKS.--Lake sampled near center at the deep hole. Lake ice-covered during February sampling. Water-quality analyses done by Wisconsin State Laboratory of Hygiene.

WATER-QUALITY DATA, FEBRUARY 18 TO JUNE 7, 2004

(Milligrams per liter unless otherwise indicated)

Date

Secchi depth (m)

Depth of sample $(\mathrm{m})$

Chlorophyll a, phytoplankton ( $\mu \mathrm{g} / \mathrm{L}$ )

Water temperature $\left({ }^{\circ} \mathrm{C}\right)$

Specific conductance $(\mu \mathrm{S} / \mathrm{cm})$

$\mathrm{pH}$

Dissolved oxygen (mg/L)

Phosphorus, total (as $\mathrm{P}$ )

Phosphorus, ortho, dissolved (as $\mathrm{P}$ )

Nitrogen, $\mathrm{NO}_{2}+\mathrm{NO}_{3}$, diss. (as $\mathrm{N}$ )

Nitrogen, ammonia, dissolved (as $\mathrm{N}$ )

Nitrogen, amm. + org., total (as N)

Nitrogen, total (as $N$ )

Color (Pt-Co. scale)

Turbidity (NTU)

Hardness, as $\mathrm{CaCO}_{3}$

Calcium, dissolved ( $\mathrm{Ca}$ )

Magnesium, dissolved (Mg)

Sodium, dissolved $(\mathrm{Na})$

Potassium, dissolved $(\mathrm{K})$

Alkalinity, as $\mathrm{CaCO}_{3}$

Sulfate, dissolved $\left(\mathrm{SO}_{4}\right)$

Chloride, dissolved (Cl)

Silica, dissolved $\left(\mathrm{SiO}_{2}\right)$

Solids, dissolved, at $180^{\circ} \mathrm{C}$

Iron, dissolved $(\mathrm{Fe})(\mu \mathrm{g} / \mathrm{L})$

Manganese, dissolved, $(\mathrm{Mn})(\mu \mathrm{g} / \mathrm{L})$

\begin{tabular}{|c|c|c|c|}
\hline \multicolumn{2}{|c|}{ Feb-18 } & \multicolumn{2}{|c|}{ Apr-12 } \\
\hline & & & \\
\hline 0.5 & 15 & 0.5 & 15 \\
\hline- & - & 2.5 & -- \\
\hline 1.0 & 2.7 & 9.0 & 8.3 \\
\hline 814 & - & 757 & 760 \\
\hline 7.9 & 7.2 & 8.2 & 7.9 \\
\hline 13.1 & 4.1 & 10.8 & 9.2 \\
\hline 0.023 & 0.039 & 0.030 & 0.036 \\
\hline- & - & $<0.002$ & - \\
\hline- & - & 0.513 & - \\
\hline- & - & 0.104 & - \\
\hline- & -- & 1.4 & - \\
\hline -- & -- & 1.9 & -- \\
\hline - & -- & 30 & - \\
\hline- & - & 1.9 & - \\
\hline- & - & 250 & - \\
\hline -- & - & 47 & - \\
\hline - & - & 32.1 & - \\
\hline - & - & 54.3 & - \\
\hline- & - & 3 & - \\
\hline - & - & 171 & - \\
\hline- & - & 46.9 & -- \\
\hline -- & - & 110 & - \\
\hline .. & -- & 0.022 & - \\
\hline- & - & 446 & -- \\
\hline - & -- & $<100$ & - \\
\hline-- & -- & $M$ & - \\
\hline
\end{tabular}

4-12-04

DISSOLVED OXYGEN (D.O.) IN MILLIGRAMS PER LITER

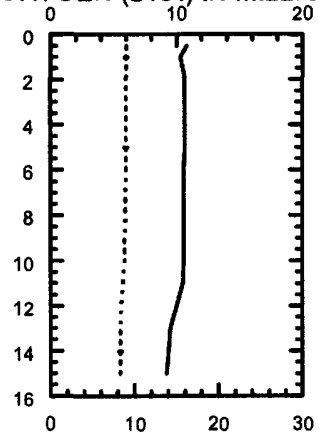

WATER TEMPERATURE (W.T.) IN DEGREES CELSIUS

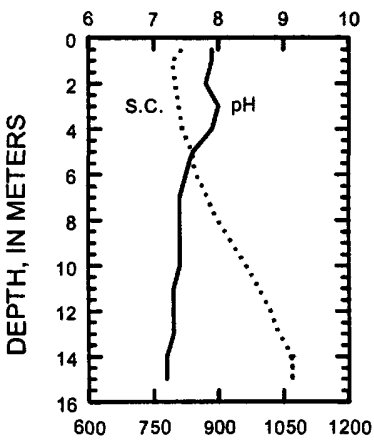

PH IN STANDARD UNITS

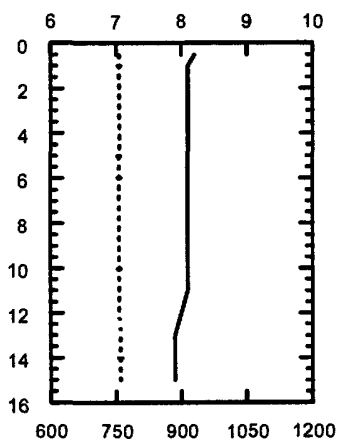

$\frac{\text { Jun-7 }}{2.1}$

15.5

13.2

793

7.2

0.4
0.305

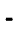

$-$

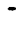

-

$-$

$-$

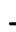

$-$

$6-7-04$

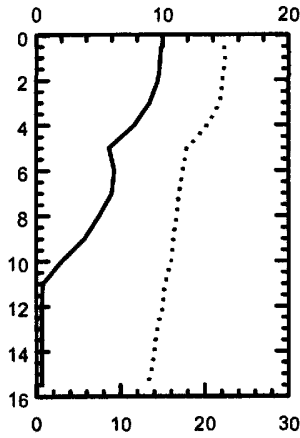

SPECIFIC CONDUCTANCE (S.C.) IN MICROSIEMENS PER CENTIMETER AT 25 DEGREES CELSIUS

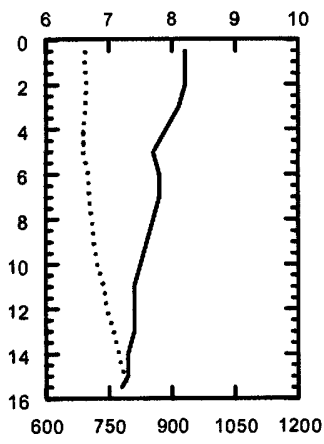


WATER-QUALITY DATA, JULY 12 TO AUGUST 16, 2004

(Milligrams per liter unless otherwise indicated)

Date

Secchi depth (m)

Depth of sample $(m)$

Chlorophyll a, phytoplankton $(\mu \mathrm{g} / \mathrm{L})$

Water temperature $\left({ }^{\circ} \mathrm{C}\right)$

Specific conductance $(\mu \mathrm{S} / \mathrm{cm})$

$\mathrm{pH}$

Dissolved oxygen ( $\mathrm{mg} / \mathrm{L})$

Phosphorus, total (as P)

Phosphorus, ortho, dissolved (as $\mathrm{P}$ )

Nitrogen, $\mathrm{NO}_{2}+\mathrm{NO}_{3}$, diss. (as $\mathrm{N}$ )

Nitrogen, ammonia, dissolved (as $\mathrm{N}$ )

Nitrogen, amm. + diss., total (as N)

$\begin{array}{ccc} & \frac{\text { Jul-12 }}{1.1} & \\ 0.5 & & 15.5 \\ 18.2 & - \\ 25.6 & 14.5 \\ 672 & 764 \\ 8.4 & 7.2 \\ 13.3 & 0.2 \\ 0.035 & 0.360 \\ <0.002 & -- \\ <0.019 & -- \\ 0.017 & - \\ 1.3 & -\end{array}$

7-12-04

DISSOLVED OXYGEN (D.O.) IN MILLIGRAMS PER LITER

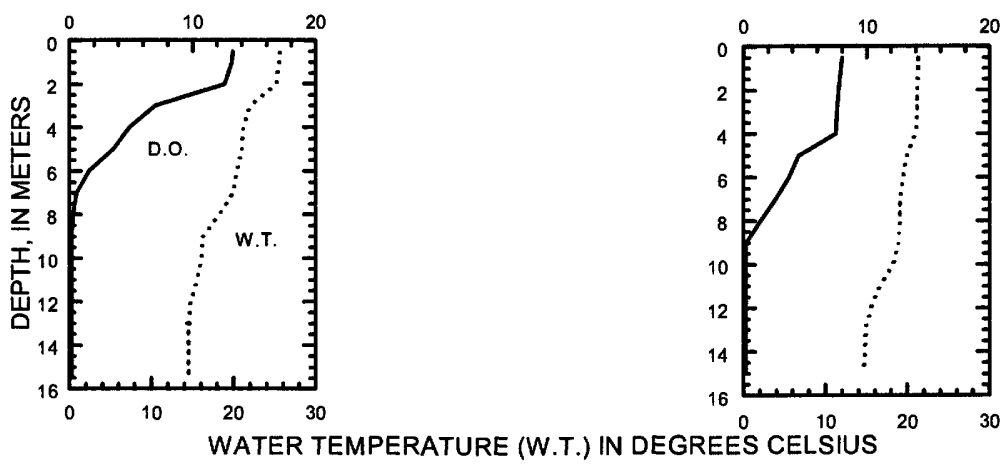

PH IN STANDARD UNITS
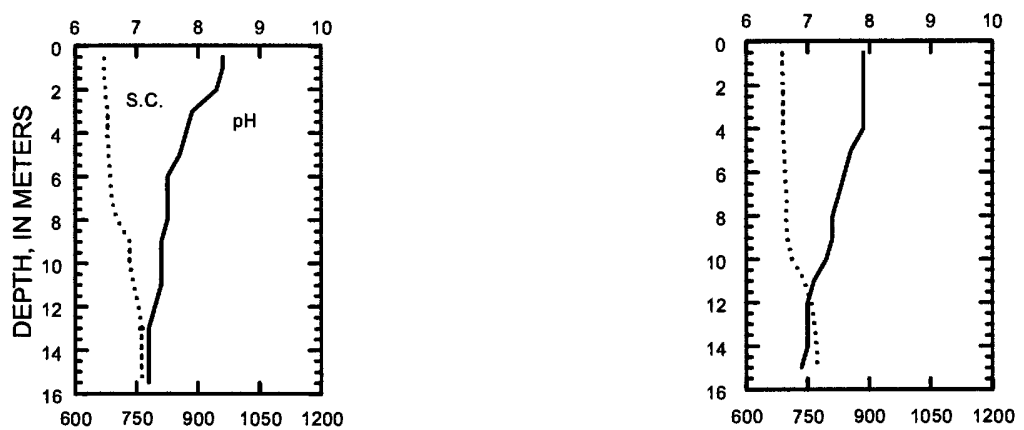

SPECIFIC CONDUCTANCE (S.C.) IN MICROSIEMENS PER CENTIMETER AT 25 DEGREES CELSIUS 

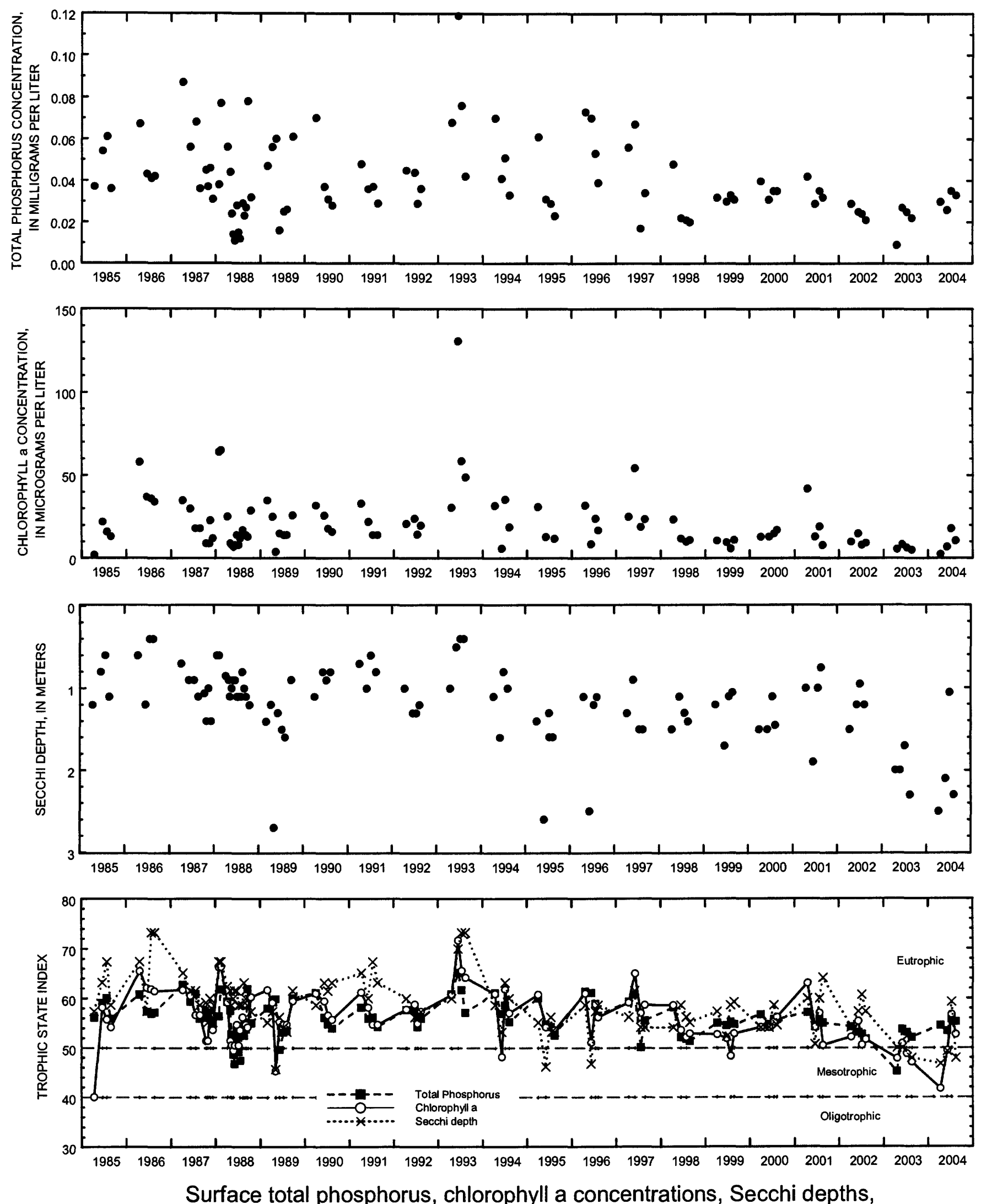
and TSI data for Wind Lake, Deep Hole, at Wind Lake, Wisconsin. 


\section{LAKE WINNEBAGO AT OSHKOSH, WI}

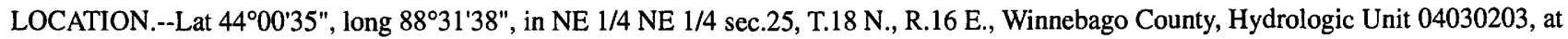
905 Bay Shore Drive, $800 \mathrm{ft}$ east of mouth of the upper Fox River.

DRAINAGE AREA.--5,880 $\mathrm{mi}^{2}$, at lake outlet at Menasha Dam. Area of Lake Winnebago, $215 \mathrm{mi}^{2}$.

PERIOD OF RECORD.--October 1938 to current year in reports of Geological Survey. Records from July 1882 to September 1938 in files of Geological Survey and U.S. Army Corps of Engineers. A report on Fox River by U.S. Army Corps of Engineers, published as House Document No. 146, 67th Congress, 2nd session, contains semi-monthly records of inflow of Lake Winnebago for the period $1896-1917$.

REVISED RECORD.--WDR WI-83-1: Drainage area.

GAGE.--Water-stage recorder. Nonrecording gage read once daily October 1938 to October 1978. Datum of gage is $745.05 \mathrm{ft}$ above mean tide at New York City (levels by U.S. Army Corps of Engineers). Datum of Deuchman gage is $745.00 \mathrm{ft}$ above mean tide at New York City.

REMARKS.--Lake elevations controlled by dams at Menasha and Neenah, which are operated in the interest of navigation. Crests of both dams are at elevation $746.73 \mathrm{ft}$. Present limits of regulation are from 21 1/4 in. above the crest of Menasha dam to crest during navigation season, plus additional $18 \mathrm{in}$. below crest during winter. Oshkosh staff gage gives true level of lake, while Deuchman gage readings are affected by loss of head in the channel between lake and dam. Data-collection platform and gage-height telemeter at station.

EXTREMES FOR PERIOD OF RECORD.--Maximum gage height observed, $5.33 \mathrm{ft}$ (Deuchman gage) Nov. 8, 1881; minimum observed, $2.00 \mathrm{ft}$ (Deuchman gage) Nov. 28, 1891.

EXTREMES FOR CURRENT YEAR.--Maximum daily mean gage height, $3.77 \mathrm{ft}$, June 19; minimum recorded, $1.62 \mathrm{ft}$, Feb. 29.

GAGE HEIGHT, FEET

WATER YEAR OCTOBER 2003 TO SEPTEMBER 2004

DAILY MEAN VALUES

\begin{tabular}{|c|c|c|c|c|c|c|c|c|c|c|c|c|}
\hline DAY & OCT & NOV & DEC & JAN & FEB & MAR & APR & MAY & JUN & JUL & AUG & SEP \\
\hline 1 & 2.57 & 2.46 & 2.39 & 2.46 & 2.08 & 1.64 & 2.02 & 2.23 & 3.15 & 3.46 & 2.97 & 3.01 \\
\hline 2 & 2.56 & 2.47 & 2.44 & 2.46 & 2.07 & 1.71 & 2.01 & 2.26 & 3.23 & 3.40 & 2.99 & 3.01 \\
\hline 3 & 2.52 & 2.57 & 2.43 & 2.46 & 2.07 & 1.74 & 2.00 & 2.29 & 3.22 & 3.34 & 3.02 & 3.01 \\
\hline 4 & 2.57 & 2.58 & 2.44 & 2.49 & 2.05 & 1.74 & 2.10 & 2.26 & 3.21 & 3.27 & 3.04 & 3.00 \\
\hline 5 & 2.57 & 2.57 & 2.45 & 2.48 & 2.02 & 1.87 & 2.11 & 2.36 & 3.19 & 3.28 & 3.01 & 2.99 \\
\hline 6 & 2.54 & 2.54 & 2.46 & 2.47 & 2.03 & 1.94 & 2.11 & 2.36 & 3.17 & 3.20 & 2.98 & \\
\hline 7 & 2.53 & 2.47 & 2.45 & 2.46 & 2.01 & 1.97 & 2.13 & 2.42 & 3.15 & 3.13 & 2.99 & 3.0 \\
\hline 8 & 2.53 & 2.48 & 2.45 & 2.44 & 1.99 & 2.00 & 2.13 & 2.54 & 3.12 & 3.08 & 2.97 & 3.0 \\
\hline 9 & 2.52 & 2.44 & 2.47 & 2.43 & 1.97 & 2.00 & 2.13 & 2.69 & 3.16 & 3.06 & 2.94 & 2.98 \\
\hline 10 & 2.52 & 2.44 & 2.53 & 2.43 & 1.95 & 2.01 & 2.17 & 2.75 & 3.19 & 3.00 & 2.95 & 2.9 \\
\hline 11 & 2.49 & 2.46 & 2.52 & 2.41 & 1.94 & 2.04 & 2.17 & 2.78 & 3.46 & 2.95 & 2.94 & 2 \\
\hline 12 & 2.51 & 2.37 & 2.56 & 2.40 & 1.92 & 2.06 & 2.13 & 2.77 & 3.55 & 2.90 & 2.96 & 2.5 \\
\hline 13 & 2.51 & 2.49 & 2.52 & 2.39 & 1.90 & 2.05 & 2.10 & 2.82 & 3.62 & 2.89 & 2.93 & 2.9 \\
\hline 14 & 2.54 & 2.52 & 2.50 & 2.37 & 1.88 & 2.08 & 2.08 & 2.87 & 3.68 & 2.93 & 2.93 & 2.9 \\
\hline 15 & 2.51 & 2.51 & 2.48 & 2.36 & 1.86 & 2.09 & 2.06 & 2.89 & 3.72 & 2.95 & 2.92 & 2.88 \\
\hline 16 & 2.50 & 2.52 & 2.47 & 2.35 & 1.84 & 2.08 & 2.02 & 2.84 & 3.71 & 2.95 & 2.90 & 2.9 \\
\hline 17 & 2.48 & 2.53 & 2.47 & 2.35 & 1.82 & 2.07 & 2.01 & 2.79 & 3.76 & 2.96 & 2.93 & 2 \\
\hline 18 & 2.46 & 2.54 & 2.46 & 2.35 & 1.80 & 2.07 & 1.98 & 2.82 & 3.76 & 2.93 & 2.92 & 2.8 \\
\hline 19 & 2.47 & 2.56 & 2.45 & 2.32 & 1.77 & 2.07 & 1.90 & 2.79 & 3.77 & 2.91 & 2.93 & 2.8 \\
\hline 20 & 2.43 & 2.56 & 2.44 & 2.30 & 1.77 & 2.05 & 2.02 & 2.81 & 3.75 & 2.92 & 2.92 & 2.8 \\
\hline 21 & 2.49 & 2.58 & 2.43 & 2.28 & 1.77 & 2.06 & 1.90 & 2.87 & 3.75 & 2.91 & 2.92 & 2. \\
\hline 22 & 2.48 & 2.59 & 2.42 & 2.26 & 1.75 & 2.02 & 1.98 & 2.88 & 3.73 & 2.94 & 2.87 & \\
\hline 23 & 2.46 & 2.66 & 2.42 & 2.25 & 1.75 & 1.99 & 1.96 & 3.02 & 3.72 & 2.94 & 2.92 & 2.8 \\
\hline 24 & 2.46 & 2.63 & 2.43 & 2.24 & 1.73 & 1.97 & 2.03 & 3.07 & 3.69 & 2.93 & 2.89 & 2.7 \\
\hline 25 & 2.46 & 2.66 & 2.43 & 2.22 & 1.70 & 1.96 & 1.99 & 3.06 & 3.68 & 2.90 & 2.89 & 2.8 \\
\hline 26 & 2.45 & 2.64 & 2.42 & 2.21 & 1.67 & 1.96 & 1.95 & 3.03 & 3.65 & 2.90 & 2.91 & 2.8 \\
\hline 27 & 2.45 & 2.60 & 2.41 & 2.19 & 1.65 & 1.95 & 2.05 & 3.01 & 3.63 & 2.91 & 3.01 & 2.8 \\
\hline 28 & 2.40 & 2.53 & 2.43 & 2.17 & 1.63 & 1.94 & 2.07 & 2.99 & 3.59 & 2.90 & 3.05 & 2.8 \\
\hline 29 & 2.43 & 2.53 & 2.44 & 2.15 & 1.62 & 1.97 & 2.11 & 2.96 & 3.54 & 2.90 & 3.03 & 2.7 \\
\hline 30 & 2.45 & 2.44 & 2.46 & 2.13 & -- & 2.02 & 2.19 & 2.98 & 3.50 & 2.94 & 2.99 & 2.7 \\
\hline 31 & 2.43 & $\ldots$ & 2.45 & 2.10 & -- & 2.02 & -.- & 3.00 & --. & 2.94 & 3.01 & \\
\hline MEAN & 2.49 & 2.53 & 2.46 & 2.33 & 1.86 & 1.97 & 2.05 & 2.75 & 3.50 & 3.02 & 2.96 & \\
\hline MAX & 2.57 & 2.66 & 2.56 & 2.49 & 2.08 & 2.09 & 2.19 & 3.07 & 3.77 & 3.46 & 3.05 & \\
\hline MIN & 2.40 & 2.37 & 2.39 & 2.10 & 1.62 & 1.64 & 1.90 & 2.23 & 3.12 & 2.89 & 2.87 & 2.7 \\
\hline
\end{tabular}




\section{LAKE WINNEBAGO NEAR STOCKBRIDGE, WI}

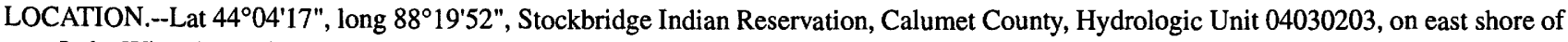
Lake Winnebago, $300 \mathrm{ft}$ south of County Highway E and $1.6 \mathrm{mi}$ west of Stockbridge.

DRAINAGE AREA.--5,880 $\mathrm{mi}^{2}$, at lake outlet at Menasha Dam. Area of Lake Winnebago, $215 \mathrm{mi}^{2}$.

PERIOD OF RECORD. - November 1982 to current year.

GAGE.--Water-stage recorder. Datum of gage is $745.05 \mathrm{ft}$ above mean tide of New York City (levels by U. S. Army Corps of Engineers).

REMARKS.--Lake elevations controlled by dams at Menasha and Neenah, which are operated in the interest of navigation. Crests of both dams are at elevation $746.73 \mathrm{ft}$. Present limits of regulation are from $211 / 4 \mathrm{in}$. above the crest of Menasha dam to crest during navigation season, plus additional $18 \mathrm{in.} \mathrm{below} \mathrm{crest} \mathrm{during} \mathrm{winter.} \mathrm{Data-collection} \mathrm{platform} \mathrm{and} \mathrm{gage-height} \mathrm{telemeter} \mathrm{at} \mathrm{station.}$

EXTREMES FOR PERIOD OF RECORD.--Maximum daily mean gage height, $3.85 \mathrm{ft}$, July 9, 11, 1993; minimum observed, $0.30 \mathrm{ft}$, Mar. 1 , 1986.

EXTREMES FOR CURRENT YEAR.--Maximum daily mean gage height, $3.81 \mathrm{ft}$, June 20; minimum recorded, $1.57 \mathrm{ft}$, Feb. 29.

\section{WATER YEAR OCTOBER 2003 TO SEPTEMBER 2004 \\ DAILY MEAN VALUES}

\begin{tabular}{|c|c|c|c|c|c|c|c|c|c|c|c|c|}
\hline DAY & OCT & NOV & DEC & JAN & FEB & MAR & APR & MAY & JUN & JUL & AUG & SEP \\
\hline 1 & 2.57 & 2.41 & 2.55 & 2.40 & 2.03 & 1.59 & 1.93 & 2.15 & 3.16 & 3.42 & 2.97 & 2.98 \\
\hline 2 & 2.54 & 2.40 & 2.40 & 2.41 & 2.01 & 1.67 & 1.95 & 2.16 & 3.17 & 3.33 & 2.99 & 2.98 \\
\hline 3 & 2.60 & 2.36 & 2.37 & 2.45 & 2.02 & 1.69 & 1.95 & 2.24 & 3.18 & 3.27 & 2.95 & 2.98 \\
\hline 4 & 2.53 & 2.52 & 2.38 & 2.42 & 1.99 & 1.69 & 2.02 & 2.28 & 3.17 & 3.27 & 2.92 & 2.98 \\
\hline 5 & 2.49 & 2.60 & 2.37 & 2.46 & 1.97 & 1.84 & 2.04 & 2.29 & 3.16 & 3.19 & 2.93 & 2.97 \\
\hline 6 & 2.48 & 2.57 & 2.39 & 2.44 & 1.98 & 1.90 & 2.06 & 2.31 & 3.14 & 3.16 & 2.96 & 3.03 \\
\hline 7 & 2.48 & 2.53 & 2.39 & 2.41 & 1.97 & 1.96 & 2.08 & 2.25 & 3.15 & 3.13 & 2.97 & 3.00 \\
\hline 8 & 2.48 & 2.43 & 2.40 & 2.39 & 1.93 & 1.96 & 2.07 & 2.45 & 3.12 & 3.10 & 2.96 & 2.91 \\
\hline 9 & 2.46 & 2.43 & 2.40 & 2.38 & 1.92 & 1.96 & 2.10 & 2.63 & 3.08 & 3.02 & 3.01 & 2.93 \\
\hline 10 & 2.46 & 2.41 & 2.44 & 2.37 & 1.90 & 1.96 & 2.09 & 2.72 & 3.06 & 2.96 & 3.05 & 2.94 \\
\hline 11 & 2.46 & 2.43 & 2.59 & 2.36 & 1.89 & 2.01 & 2.06 & 2.71 & 3.29 & 2.91 & 3.02 & 2.94 \\
\hline 12 & 2.54 & 2.52 & 2.51 & 2.36 & 1.87 & 2.01 & 2.04 & 2.73 & 3.51 & 2.87 & 2.90 & 2.93 \\
\hline 13 & 2.49 & 2.61 & 2.46 & 2.34 & 1.84 & 1.99 & 2.03 & 2.79 & 3.60 & 2.88 & 2.88 & 2.91 \\
\hline 14 & 2.48 & 2.49 & 2.45 & 2.32 & 1.82 & 2.05 & 2.02 & 2.82 & 3.66 & 2.91 & 2.89 & 2.91 \\
\hline 15 & 2.53 & 2.45 & 2.42 & 2.30 & 1.81 & 2.05 & 1.98 & 2.83 & 3.68 & 2.93 & 2.88 & 2.95 \\
\hline 16 & 2.49 & 2.46 & 2.44 & 2.29 & 1.79 & 2.03 & 1.95 & 2.80 & 3.69 & 2.91 & 2.89 & 2.94 \\
\hline 17 & 2.46 & 2.46 & 2.43 & 2.30 & 1.77 & 2.02 & 1.93 & 2.79 & 3.72 & 2.87 & 2.91 & 2.86 \\
\hline 18 & 2.45 & 2.49 & 2.42 & 2.30 & 1.74 & 2.02 & 2.03 & 2.75 & 3.77 & 2.89 & 2.95 & 2.85 \\
\hline 19 & 2.40 & 2.56 & 2.41 & 2.28 & 1.72 & 2.01 & 2.01 & 2.72 & 3.76 & 2.91 & 2.95 & 2.83 \\
\hline 20 & 2.42 & 2.54 & 2.38 & 2.25 & 1.73 & 2.02 & 1.85 & 2.75 & 3.81 & 2.89 & 2.91 & 2.83 \\
\hline 21 & 2.43 & 2.49 & 2.37 & 2.23 & 1.73 & 2.01 & 1.89 & 2.73 & 3.77 & 2.90 & 2.89 & 2.83 \\
\hline 22 & 2.41 & 2.40 & 2.37 & 2.22 & 1.69 & 1.97 & 1.88 & 2.81 & 3.76 & 2.92 & 2.91 & 2.82 \\
\hline 23 & 2.41 & 2.65 & 2.38 & 2.20 & 1.69 & 1.93 & 1.91 & 2.90 & 3.76 & 2.88 & 2.81 & 2.80 \\
\hline 24 & 2.41 & 2.89 & 2.38 & 2.19 & 1.67 & 1.90 & 1.88 & 3.04 & 3.77 & 2.86 & 2.82 & 2.86 \\
\hline 25 & 2.42 & 2.73 & 2.38 & 2.17 & 1.65 & 1.89 & 1.94 & 3.03 & 3.72 & 2.86 & 2.86 & 2.81 \\
\hline 26 & 2.45 & 2.61 & 2.37 & 2.15 & 1.62 & 1.91 & 2.05 & 3.02 & 3.66 & 2.86 & 2.88 & 2.78 \\
\hline 27 & 2.41 & 2.56 & 2.35 & 2.15 & 1.60 & 1.89 & 2.04 & 2.97 & 3.62 & 2.87 & 2.99 & 2.75 \\
\hline 28 & 2.44 & 2.58 & 2.37 & 2.13 & 1.58 & 1.89 & 2.08 & 2.93 & 3.61 & 2.90 & 2.95 & 2.68 \\
\hline 29 & 2.44 & 2.56 & 2.39 & 2.10 & 1.57 & 1.95 & 2.12 & 2.89 & 3.56 & 2.92 & 2.96 & 2.74 \\
\hline 30 & 2.35 & 2.49 & 2.41 & 2.08 & - & 1.91 & 2.10 & 2.81 & 3.50 & 2.91 & 2.98 & 2.74 \\
\hline 31 & 2.40 & - & 2.41 & 2.06 & -- & 1.92 & - & 3.02 & - & 2.94 & 2.97 & - \\
\hline MEAN & 2.46 & 2.52 & 2.41 & 2.29 & 1.81 & 1.92 & 2.00 & 2.69 & 3.49 & 2.99 & 2.93 & 2.88 \\
\hline MAX & 2.60 & 2.89 & 2.59 & 2.46 & 2.03 & 2.05 & 2.12 & 3.04 & 3.81 & 3.42 & 3.05 & 3.03 \\
\hline MIN & 2.35 & 2.36 & 2.35 & 2.06 & 1.57 & 1.59 & 1.85 & 2.15 & 3.06 & 2.86 & 2.81 & 2.68 \\
\hline
\end{tabular}




\section{WISCONSIN DISTRICT PUBLICATIONS PERTAINING TO LAKES}

The reports published in a U.S. Geological Survey series are for sale by the U.S. Geological Survey, Box 25286, Federal Center, Denver, CO 80225. Prepayment is required. Remittance should be sent by check or money order payable to the U.S. Geological Survey. For more information and prices on USGS products, contact representatives at 1-888-ASK-USGS. For access to the new Publications Warehouse, go to http://pubs.usgs.gov/ or link from the complete list of USGS publications and products at http://www.usgs.gov/pub/prod/. Reprints of journal articles may be obtained by writing directly to the author at U.S. Geological Survey, Wisconsin Water Science Center, 8505 Research Way, Middleton, WI 53562.

2004

Dupre, D.H., and Robertson, D.M., 2004, Water quality of Nippersink Creek and Wonder Lake, McHenry County, Illinois, 1994-2001: U.S. Geological Survey Scientific Investigations Report 2004-5085.

Rose, W.J., Robertson, D.M., and Mergener, E.A., 2004, Water Quality, Hydrology, and the Effects of Changes in Phosphorus Loading to Pike Lake, Washington County, Wisconsin, with Special Emphasis on Inlet-to-Outlet Short-Circuiting: U.S. Geological Survey Scientific Investigations Report 2004-5141, 32 p.

2003

Dunning, C.P., Thomas, J.C., and Lin, Y-F., 2003, Simulation of the Shallow Aquifer in the Vicinity of Silver Lake, Washington County, Wisconsin, Using Analytic Elements: U.S. Geological Survey Water-Resources Investigations Report 02-4204, 29 p.

Fitzpatrick, F.A., Garrison, P.J., Fitzgerald, S.A., and Elder, J.F., 2003, Nutrient, Trace-Element, and ecological history of Musky Bay, Lac Courte Oreilles, Wisconsin, as inferred from sediment cores: U.S. Geological Survey Water-Resources Investigations Report 02-4225, $141 \mathrm{p}$.

Fitzpatrick, F.A., and Peppler, M.C., 2003, Sedimentation and sediment chemistry, Neopit Mill Pond, Menominee Indian Reservation, Wisconsin, 2001: U.S. Geological Survey Open-File Report 03-23, $58 \mathrm{p}$.

Garn, H.S., Elder, J.F., and Robertson, D.M., 2003, Why study lakes? An overview of U.S. Geological Survey lake studies in Wisconsin: U.S. Geological Survey Fact Sheet FS-063-03, 8 p.

Graczyk, D.G., Hunt, R.J., Greb, S.R., Buchwald, C.A., and Krohelski, J.T., 2003, Hydrology, water quality, and yields, from near-shore flows to four lakes in northern Wisconsin, 19992001, U.S. Geological Survey Water-Resources Investigations Report 03-4144, 64 p.

Hunt, R.J., 2003, Ground water-lake interaction modeling using the LAK3 Package for MODFLOW2000: Ground Water, vol. 41, no. 2, p. 114-118. 
Hunt, R.J., Haitjema, H.M., Krohelski, J.T., and Feinstein, D.T., 2003, Simulating ground water-lake interactions: approaches and insights: Ground Water, vol. 41, no. 22, p. 227-237.

Hunt, R.J., Pint, C.D., and Anderson, M.P., 2003, Using Diverse Data Types to Calibrate a Watershed Model of the Trout Lake Basin, Northern Wisconsin, p.600-604, In: MODFLOW and More 2003: Understanding through Modeling: Proceedings of the 5th International Conference of the International Ground Water Modeling Center. Golden, CO: Colorado School of Mines.

Hunt, R.J., Saad, D.A., and Chapel, D.M., 2003, Numerical Simulation of Ground-Water Flow in La Crosse County, Wisconsin and into Nearby Pools of the Mississippi River. U.S. Geological Survey Water-Resources Investigations Report 03-4154. $36 \mathrm{p}$.

John, R., Pint, C.D., Anderson, M.P., and Hunt, R.J., 2003, The Effects of Potential Climate Change on Lake Levels and Lake Capture Zones, p. 212-216, In MODFLOW and More 2003: Understanding through Modeling: Proceedings of the 5th International Conference of the International Ground Water Modeling Center. Golden, CO: Colorado School of Mines.

Magnuson, J.J., Krohelski, J.T., Kunkel, K.E., and Robertson, D.M., 2003, Wisconsin's waters and climate: historical changes and possible futures: In Wisconsin's waters: a confluence of perspectives. Transactions of the Wisconsin Academy, p. 23-36.

Pint, C.D., Hunt, R.J., and Anderson, M.P., 2003, Flow path delineation and ground water age, Allequash Basin, Wisconsin: Ground Water, vol. 41, no. 7, p. 895-902.

Robertson, D.M., Rose, W.J., and Garn, H.S., 2003, Water quality and the effects of changes in phosphorus loading, Red Cedar Lakes, Barron and Washburn Counties, Wisconsin: U.S. Geological Survey Water-Resources Investigations Report 03-4238, $42 \mathrm{p}$.

Robertson, D.M., Rose, W.J., and Saad, D.A., 2003, Water quality and the effects of changes in phosphorus loading to Muskellunge Lake, Vilas County, Wisconsin: U.S. Geological Survey Water-Resources Investigations Report 03-4011, 18 p.

2002

Anderson, M.P., Hunt, R.J., Krohelski, J.T., and Chung, K., 2002, Using high hydraulic conductivity nodes to simulate seepage lakes: Ground Water 40(2), p. 119-124.

Garn, H. S., 2002, Effects of lawn fertilizer on nutrient concentration in runoff from lakeshore lawns, Lauderdale Lakes, Wisconsin: USGS Water-Resources Investigations Report 02-4130, $6 \mathrm{p}$.

Kelson, V.A., Hunt, R.J., and Haitjema, H.M., 2002, Improving a regional model using reduced complexity and parameter estimation: Ground Water 40(2), p. 138-149.

Krohelski, J.T., Lin, Y., Rose, W.J., and Hunt, R.J., 2002, Simulation of Fish, Mud and Crystal Lakes and the shallow ground-water system, Dane County, Wisconsin: USGS Water-Resources Investigations Report 02-20. 
Krohelski, J.T., Rose, W.J., and Hunt, R.J., 2002, Hydrologic investigation of Powell Marsh and its relationship to Dead Pike Lake Vilas County, Wisconsin: USGS WaterResources Investigations Report 02-4034.

Madenjian, C.P., Fahenstiel, G.L., Johengen, T.H., Nalepa, T.F., Venderploeg, H.A., Fleischer, G.W., Schneeberger, P.J., Benjamin, D.M., Smith, E.B., Bence, J.R., Rutherford, E.S., Lavis, D.S., Robertson, D.M., Jude, D.J., and Ebener, M.P., 2002, Dynamics of the Lake Michigan food web, 1970-2000: Canadian Journal of Fisheries and Aquatic Sciences, Vol. 59, p. 736-753.

Robertson, D.M., Goddard, G.L, Mergerer, E.A., Rose, W.J., and Garrison, P.J., 2002, Hydrology and water quality of Geneva Lake, Walworth County, Wisconsin: U.S. Geological Survey Water Resources Investigations Report 02-4039, 73 p.

Robertson, D.M., and Lenz, B.N., 2002, Response of the St. Croix River Pools, Wisconsin and Minnesota, to various phosphorus-loading scenarios: U.S. Geological Survey Water-Resources Investigations Report 02-4181, $36 \mathrm{p}$.

2001

Hunt, R.J., Bradbury, K.R., and Krohelski, J.T., 2001, The effects of large-scale pumping and diversion on the water resources in Dane County, Wisconsin: USGS Fact Sheet FS-127-01, 4 p.

Hunt, R.J., Haitjema, H.M., Krohelski, J.T., and Feinstein, D.T., 2001, Simulating groundwater-lake interactions with models: MODFLOW and analytic element approaches. p. 328-334 in MODFLOW 2001 and Other Modeling Odysseys: Proceedings of the 4th International Conference of the International Ground Water Modeling Center. Golden, CO: Colorado School of Mines.

Lin, Y., Krohelski, J.T., and Hunt, R.J., 2001, Simulation of lake stage in two seepage lakes in southcentral Wisconsin using the LAK3 package for MODFLOW. p. 411-417 in MODFLOW 2001 and Other Modeling. Odysseys: Proceedings of the 4th International Conference of the International Ground Water Modeling Center. Golden, CO: Colorado School of Mines.

2000

Elder, J.F., Robertson, D.M., and Garrison, P.J., 2000, Chemical composition of surficial deposits in Geneva Lake, Wisconsin: U.S. Geological Survey Fact Sheet FS-121-00.

Elder J.F., Rybicki, N.B., Carter, V.P., and Weintraub, V., 2000, Sources and yields of dissolved carbon in northern Wisconsin stream catchments with differing amounts of peatland: Wetlands, Vol. 20, No. 1, p.113-125.

Hunt, R.J., Graczyk, D.J., and Rose, W.J., 2000, Water flows in the Necedah National Wildlife Refuge: U.S. Geological Survey Fact Sheet FS-068-00, 4 p.

Hunt, R.J., Lin, J., Krohelski, J.T., and Juckem, P.F., 2000, Simulation of the shallow hydrologic system in the vicinity of Middle Genesee Lake, Wisconsin, using analytic 
elements and parameter estimation: U.S. Geological Survey Water Resources Investigations Report 00-4136,16 p.

Lathrop, R.C., Carpenter, S.R., and Robertson, D.M., 2000, Interacting factors causing exceptional water clarity in Lakes Mendota and Monona, Wisconsin, Proc. of the International Limnological Society, SIL, Dublin, Ireland, August, 1998.

Magnuson, J.J., Robertson, D.M. Wynne, R.H., Benson, B.J., Livigstone, D.M., Arai, T., Assel, R.A., Barry, R.G., Card, V., Kuusisto, E., Granin, N.G., Prowse, T.D., Stewart, K.M., and Vuglinski, V.S., 2000, Historical Trends in Lake and River Ice Cover in the Northern Hemisphere: Science, Vol. 289, No. 5485, p. 1743-1746.

Magnuson, J.J., Wynne, R.H., Benson, B.J., and Robertson, D.M., 2000, Lake and river ice as a powerful indicator of past and present climates, Proc. of the International Limnological Society, SIL, Dublin, Ireland, August, 1998.

Robertson, D.M., 2000, One-dimensional simulation of stratification and dissolved oxygen in McCook Reservoir, Illinois: U.S. Geological Survey Water Resources Investigations Report 00-4258, $17 \mathrm{p}$.

Robertson, D.M., Goddard, G.L., Helsel, D.R., and MacKinnon, K.L., 2000, Rehabilitation of Delavan Lake, Wisconsin: Lake and Reservoir Management, Vol. 20, No. 3, p. 155-176.

Robertson, D.M., and Rose, W.J., 2000, Hydrology, water quality, and phosphorus loading of Little St. Germain Lake, Vilas County, Wisconsin: U.S. Geological Survey Water Resources Investigations Report 00-4209, 8 p.

Robertson, D.M., Wynne, R.H., and Chang, W.Y.B., 2000, Variability in ice cover across the northern hemisphere during the 1900's associated with El Nino events, Proceedings of the International Limnological Society, SIL, Dublin, Ireland, August, 1998.

Saad, D.A., and Robertson, D.M., 2000, Water-resources-related information for the St. Croix Reservation and vicinity, Wisconsin: U.S. Geological Survey Water Resources Investigations Report 00-4133, $65 \mathrm{p}$.

Johnson, G.P., Hornewer, N.J., Robertson, D.M., and Olson, D.T., 2000, Methodology, data collection, and data analysis for determination of water-mixing patterns induced by aerators and mixers, U.S. Geological Survey Water Resources Investigations Report 00-4101, $72 \mathrm{p}$.

Grannemann, N.G., Hunt, R.J., Nicholas, J.R. Reilly, T.E., and Winter, T.C., 2000, The importance of ground water to the Great Lakes Region: U.S. Geological Survey Water Resources Investigations Report 00-4008, $12 \mathrm{p}$.

Lathrop, R.C., Carpenter, S.R., and Robertson, D.M., 1999, Summer water clarity responses to phosphorus, Daphnia grazing, and internal mixing in Lake Mendota, Limnology and Oceanography, Vol. 44 (1), p. 137-146. 
Krohelski, J.T., Feinstein, D.T., and Lenz, B.N., 1999, Simulation of stage and hydrologic budget for Shell Lake, Washburn County, Wisconsin, U.S. Geological Survey WaterResources Investigations Report 99-4209, 23 p.

Panuska, J.C., and Robertson, D.M., 1999, Estimating phosphorus concentrations following alum treatment using apparent settling velocities, Lakes and Reservoir Management, Vol. 15 (1), p. 28-38.

\section{8}

Hunt, R.J, Anderson, M.P., and Kelson, V.A., 1998, Improving a complex finite-difference ground water flow model through the use of an analytic element screening model, Ground Water, Vol. 36, No. 6, p. 1011-1017.

Hunt, R.J, Anderson, M.P., and Kelson, V.A., 1998, Linking an analytic element flow code to MODFLOW - Implementation and benefits. p. 477-504 in MODFLOW'98: Proceedings of the 3rd International Conference of the International Ground Water Center. Golden, CO: Colorado School of Mines.

Krabbenhoft, D.P., Gilmour, C.C., Benoit, J.M., Babiarz, C.L., Andren, A.W., and Hurley, J.P., 1998, Methyl mercury dynamics in littoral sediments of a temperate lake, Canadian Journal of Fisheries and Aquatic Sciences, Vol. 55, p. 835-844.

Robertson, D.M., Elder, J.F., Goddard, G.L., and James, W.F., 1998, Dynamics in phosphorus retention in wetlands upstream of Delavan Lake, Wisconsin, Lakes and Reservoir Management, Vol. 14, No. 4, p. 466-477.

Rose, W.J., and Robertson, D.M., 1998, Hydrology, water quality, and phosphorus loading of Kirby Lake, Barron County, Wisconsin, U.S. Geological Survey Fact Sheet FS-066$98,4 \mathrm{p}$.

Walker, J.F. and Krabbenhoft, D.P., 1998, Groundwater and surface-water interactions in riparian and lake-dominated systems, in Kendall, C. and McDonnell, J.J. eds., Isotope tracers in catchment hydrology, Elsevier Publishing, New York, 839 P.

1997

Elder, J.F., Manion, B.J., and Goddard, G.L., 1997, Mesocosm experiments to assess factors affecting phosphorus retention and release in an extended Wisconsin wetland: U.S. Geological Survey Water-Resources Investigations Report 97-4272, 14 p.

Goddard, G.L., and Elder, J.F., 1997, Retention of sediments and nutrients in Jackson Creek Wetland near Delavan Lake, Wisconsin, 1993-95, U.S. Geological Survey Water-Resources Investigations Report 97-4014, 22 p.

Hornewer, N.J., Johnson, G.P., Robertson, D.M. and Hondzo, M., 1997, Field-scale tests for determining mixing patterns associated with coarse-bubble air diffuser configurations, Egan Quarry, Illinois, In: Environmental and Coastal Hydraulics: Protecting the Aquatic Habitat, Proceedings of the International Association of Hydraulic Research, San Francisco, CA, USA, p. 57-63. 
Robertson, D.M., 1997, Regionalized loads of sediment and phosphorus to Lakes Michigan and Superior - High flow and long-term average, Journal of Great Lakes Research, Vol. 23, p. 416-439.

1996

Anderson, W.L., Robertson, D.M., and Magnuson, J.J., 1996, Evidence of recent warming and El Nino-related variation in ice breakup of Wisconsin lakes, Limnology and Oceanography, Vol. 41, p. 815-821.

Elder, J.F. and Goddard, G.L., 1996, Sediment and nutrient trapping efficiency of a constructed wetland near Delavan Lake, Wisconsin, 1993-1995: U.S. Geological Survey Fact Sheet FS-232-96.

Garn, H.S., Olson, D.L., Seidel, T.L., and Rose, W.J., 1996, Hydrology and water quality of Lauderdale Lakes, Walworth County, Wisconsin, 1993-94: U.S. Geological Survey Water-Resources Investigations Report 96-4235, $29 \mathrm{p}$.

Hunt, R.J. and Krohelski, J.T., 1996, The application of an analytical element model to investigate groundwater-lake interactions at Pretty Lake, Wisconsin, Lake and Reservoir Management, Vol. 12, p. 487-495.

Imberger, J., Robertson, D.M., and Boland, K., 1996, Lake Number: A quantitative indicator of mixing to be used in water quality management. Scientific Impeller, Solna, Sweden, No. 4, p. 9-15.

Kammerer, P.A., Jr., 1996, Hydrology and water quality of Park Lake, South-central Wisconsin: U.S. Geological Survey Fact Sheet FS-197-96.

Robertson, D.M., Field, S.J, Elder, J.F., Goddard, G.L., and James, W.F., 1996, Phosphorus dynamics of Delavan Lake Inlet in southeastern Wisconsin. U.S. Geological Survey Water-Resources Investigations Report 96-4160, 18 p.

1995

Assel, R.A. and Robertson, D.M. 1995, Changes in winter air temperatures near Lake Michigan during 1851 - 1993, as determined from regional lake-ice records, Limnology and Oceanography, v. 40, p 165-176.

Assel, R.A., Robertson, D.M., Hoff, M.H., and Selgeby, J.H., 1995, Climatic-change implications from long-term (1823-1994) ice records near the Laurentian Great Lakes: Annals of Glaciology, v. 21, p. 383-386.

Krabbenhoft, D.P., and Webster, K.E., 1995, Transient hydrogeological controls on the chemistry of a seepage lake: Water Resources Research, v. 31, no. 9, p. 2295-2305.

Krohelski, J.T. and Batten, W.G., 1995, Simulation of stage and the hydrologic budget of Devils Lake, Sauk County, Wisconsin: U.S. Geological Survey Open-File Report 94$348,22 \mathrm{p}$. 
Wentz, D.A., Rose, W.J., and Webster, K.E., 1995, Long-term hydrologic and biogeochemical responses of a soft water seepage lake in north central Wisconsin: Water Resources Research, v. 31, no. 1, p 199-212.

1994

Elder, J.F., 1994, Distribution and grain-size partitioning of metals in bottom sediments of an experimentally acidified lake: Water Resources Bulletin, v. 30, no. 2, p. 251-259.

Goddard, G.L., and Field, S.J., 1994, Hydrology and water quality of Whitewater and Rice Lakes in southeastern Wisconsin, 1990-91: U.S. Geological Survey Water-Resources Investigations Report 94-4101, $36 \mathrm{p}$.

Greb, S.R., and Wentz, D.A., 1994, Chemical budgets, in Klepinger, K.E., ed., RILWAS 1983-86: Wisconsin Regional Integrated Lake Watershed Acidification Study, Volume 1: Madison, Wisconsin Department of Natural Resources, PUBL-RS-909-94, Chapter $7,20 \mathrm{p}$.

Hurley, J.P., Krabbenhoft, D.P., Babiarz, C.L., and Andren, A.W., 1994, Cycling processes of mercury across sediment/water interfaces in seepage lakes, in Baker, L.A. ed., Environmental Chemistry of Lakes and Reservoirs: Advances in Chemistry Series, American Chemical Society, Washington, D.C., p. 426-449.

Krabbenhoft, D.P., Bowser, C.J., Kendall, C., and Gat, J.R., 1994, Use of oxygen-19 and deuterium to assess the hydrology of ground-water/lake systems, in Baker, L.A. ed., Environmental Chemistry of Lakes and Reservoirs: Advances in Chemistry Series, American Chemical Society, Washington, D.C., p. 67-90.

Robertson, D.M., Anderson, W., and Magnuson, J.J., 1994, Relations between El Nino/ Southern Oscillation events and the climate and ice cover of lakes in Wisconsin. P 4857, in Greenland, D. ed., El Nino and Long-Term Ecological Research (LTER) Sites: Publication No. 18. LTER Network Office: University of Washington, Seattle, WA, USA., $57 \mathrm{p}$.

Robertson, D.M. and Imberger, J. 1994, Lake Number, a quantitative indicator of mixing used to estimate changes in dissolved oxygen, Internationale Revue der gesamten Hydrobiologie, v. 79, p. 159-176.

Watras, C.J., Bloom, N.S., Hurley, J.P., Fitzgerald, W.F., Andren, A.W., Krabbenhoft, D.P., and Porcella, D.B., 1994, Sources and fates of mercury and methylmercury in Wisconsin lakes, in Watras and Huckabee eds., Mercury as a Global Pollutant: Intergration and Synthesis, Lewis Pub., Chelsea, MI., p 153-177.

Wentz, D.A., 1994, Chemistry of snowpack and ground water, in Klepinger, K.E., ed., RILWAS 1983-86: Wisconsin Regional Integrated Lake Watershed Acidification Study, Volume 1: Madison, Wisconsin Department of Natural Resources, PUBL-RS909-94, Chapter 6, $45 \mathrm{p}$.

Wentz, D.A., Krohelski, J.T., and Rose, W.J., 1994, Hydrology, in Klepinger, K.E., ed., RILWAS 1983-86: Wisconsin Regional Integrated Lake Watershed Acidification 
Study, Volume 1: Madison, Wisconsin Department of Natural Resources, PUBL-RS909-94, Chapter 7, 74 p.

\section{3}

Field, S.J., 1993, Hydrology and water quality of Powers Lake, southeastern Wisconsin: U.S. Geological Survey Water-Resources Investigations Report 90-4126, 36 p.

Field, S.J., 1993, Hydrology and water quality of Wind Lake in southeastern Wisconsin: U.S. Geological Survey Water-Resources Investigations Report 91-4107, 61 p.

House, L.B., 1993, Simulation of the effects of hypothetical residential development on water levels in Graber Pond, Middleton, Wisconsin: U.S. Geological Survey WaterResources Investigations Report 92-4029, $10 \mathrm{p}$.

House, L.B., Waschbusch, R.J., and Hughes, P.E., 1993, Water quality of an urban wet detention pond in Madison, Wisconsin, 1987-88: U.S. Geological Survey Open-File Report 93-172, $57 \mathrm{p}$.

Hughes, P.E., 1993, Hydrology, water quality, trophic status, and aquatic plants of Fowler Lake, Wisconsin: U.S. Geological Survey Water-Resources Investigations Report 914076, $44 \mathrm{p}$.

Rose, W.J., 1993, Hydrology of Little Rock Lake in Vilas County, north-central Wisconsin: U.S. Geological Survey Water-Resources Investigations Report 93-4139, $22 \mathrm{p}$.

Rose, W.J., 1993, Water and phosphorus budgets and trophic state, Balsam Lake, northwestern Wisconsin, 1987-1989: U.S. Geological Survey Water-Resources Investigations Report 91-4125, 28 p.

1992

Elder, J.F., Krabbenhoft, D.P, and Walker, J.F., 1992, Water, energy, and biogeochemical budgets (WEBB) program: Data availability and research at the northern temperate lakes site, Wisconsin: U.S. Geological Survey Open-File Report 92-48, 15 p.

Krabbenhoft, D.P., and Babiarz, C.L., 1992, Role of groundwater transport in aquatic mercury cycling: Water Resources Research, vol. 28, no. 12, p. 3119-3128.

Krabbenhoft, D.P., and Krohelski, J.T., 1992, Data on water quality, lake sediment, and lake-level fluctuation, St. Croix Indian Reservation, Wisconsin, 1981-87: U.S. Geological Survey Open-File Report 92-26, 53 p.

Robertson, D.M., Ragotzkie, R.A., and Magnuson, J.J. 1992, Lake ice records used to detect historical and future climatic changes: Climatic Change, v. 21, p. 407-427.

1991

Wentz, D.A., and Rose, W.J., 1991, Hydrology of Lakes Clara and Vandercook in NorthCentral Wisconsin: U.S. Geological Survey Water-Resources Investigations Report 89-4204, 24 p. 
Watras, C.J., Andre, A.W., Bloom, N.S., Fitzgerald, W.F., Hurley, J.P., Krabbenhoft, D.P., Rada, R.G., Wiener, J.G., 1991, Mercury in temperate lakes: a mechanistic field study: Verhandlungen Internat. Verein. Limnologie, 24, p. 2199-2202.

\section{Pre-1990}

Walker, J.F., Pickard, S.A., and Sonzogni, W.C., 1989 Spreadsheet watershed modeling for nonpoint-source pollution management in a Wisconsin basin: Water Resources Bulletin, v. 25, no. 1, p. 139-147.

Wentz, D.A., Garrison, P.J., and Bockheim, J.G., 1989, Section 7-Chemical input-output budgets, in Knauer, D., and Brouwer, S.A., eds., The Wisconsin Regional Integrated Lake-Watershed Acidification Study (RILWAS): 1981-1983: Palo Alto, California, Electric Power Research Institute Report EA-6214, p. 7-1 to 7-30.

Wentz, D.A., and Rose, W.J., 1989, Interrelationships among hydrologic-budget components of a northern Wisconsin seepage lake and implications for acid-deposition modeling: Archives of Environmental Contamination and Toxicology, v. 18, p. 147155.

Wentz, D.A., Rose, W.J., and Krohelski, J.T., 1989, Section 5-Hydrologic component, in Knauer, D., and Brouwer, S.A., eds., The Wisconsin Regional Integrated LakeWatershed Acidification Study (RILWAS): 1981-1983: Palo Alto, California, Electric Power Research Institute Report EA-6214, p. 5-1 to 5-77.

Field, S.J., and Duerk, M.D., 1988, Hydrology and water quality of Delavan Lake in southeastern Wisconsin: U.S. Geological Survey Water-Resources Investigations Report 87-4168, $61 \mathrm{p}$.

Krug, W.R., Ostenso, N.A., and Krohelski, J.T., 1988, Prediction of the effects of mine dewatering on four lakes near Crandon, Wisconsin, by use of a water-budget model: U.S. Geological Survey Open-File Report 87-471, 63 p.

Wentz, D.A., Krohelski, J.T., Rose, W.J., Bockheim, J.G., Garrison, P.J., Knauer, D.R., and Goldstein, R.A., 1987, Hydrologic and chemical budgets of Wisconsin lakes, in Perrry, R., Harrison, R.M., Bel, J.N.B., and Lester, J.N., eds., Acid Rain: Scientific and Technical Advances, Selper Ltd., London, p. 309-316.

House, L.B., 1986, Stage fluctuations of Wisconsin Lakes: Wisconsin Geological and Natural History Survey Information Circular No. 49, 84 p.

House, L.B., 1984, Effects of urbanization on three ponds in Middleton, Wisconsin: U.S. Geological Survey Water-Resources Investigations Report 84-4051, 17 p.

Krug, W.R., and House, L.B., 1984, Evaluation of alternative reservoir-management practices in the Rock River basin, Wisconsin: U.S. Geological Survey WaterResources Investigations Report 83-4186, $21 \mathrm{p}$.

House, L.B., 1981, An assessment of streamflow, water quality, and the effects of construction on impoundment on Bridge Creek at Augusta, Wisconsin: U.S. Geological Survey Water-Resources Investigations Open-File Report 81-1192, 25 p. 
Krug, W.R., 1981, Hydrologic effects of proposed changes in management practices, Winnebago Pool, Wisconsin: U.S. Geological Survey Water-Resources Investigations $80-107,19 \mathrm{p}$.

Batten, W.G., and Hindall, S.M., 1980, Sediment deposition in the White River Reservoir, northwestern Wisconsin: U.S. Geological Survey Water-Supply Paper 2069, 30 p.

Novitzki, R.P., and Holmstrom, B.K., 1979, Monthly and annual water budgets of Lake Wingra, Madison, Wisconsin, 1971-77: U.S. Geological Survey Water-Resources Investigations $\quad 79-100,31 \mathrm{p}$.

Rose, W.J., 1977, Hydrologic considerations associated with dredging spring ponds in Wisconsin: U.S. Geological Survey Water-Resources Investigations 77-18, 35 p.

Oakes, E.L., Hendrickson, G.E., and Zuehls, E.E., 1975, Hydrology of the Lake Wingra basin, Dane County, Wisconsin: U.S. Geological Survey Water-Resources Investigations $17-75, \quad 31 \mathrm{p}$.

Novitzki, R.P., 1971, Hydrologic investigations of Heart Lake, Green Lake County, Wisconsin: U.S. Geological Survey Administrative Report, 9 p. 


\section{APPENDIX \\ Wisconsin Lakes Team \\ Quality-Assurance Plan}

Most lake studies and monitoring programs that are conducted by the USGS Wisconsin District entail water sampling and analysis to determine water quality and biological productivity. Because all sampling and analysis is subject to error and random variability, a certain proportion of the sampling effort should include quality-assurance samples. These samples are collected and/or prepared solely for the purpose of assessing the magnitude of error and random variability so that the accuracy and precision of all data can be evaluated. The plan for this quality-assurance sampling is described below.

Three types of QA/QC samples are collected:

\section{blanks}

provide information about accuracy and errors due to treatment or reagents

replicates

provide information about precision (variability)

standard additions (spikes)

provide information about accuracy and matrix interferences

\section{Blank Sampling}

B1. A preservation blank is prepared for each month of lake sampling. This consists of deionized water or norganic blank water, to which is added any reagents or preservatives that are normally added to natural water samples. The blank is not taken to the field, but is shipped to the laboratory for analysis along with the natural water samples.

This blank sample is analyzed for the Nutrient Group ${ }^{1}$ and chlorophyll-a.

B2. At one randomly-chosen lake each month, a field blank is prepared. This consists of deionized water or inorganic blank water treated exactly the same as regular samples. During winter, the field blank is analyzed for total phosphorus (TP) only; during summer, it is analyzed for TP and chlorophyll-a, and in the spring, it is analyzed for the Nutrient Group and chlorophyll-a.

\footnotetext{
${ }^{1}$ Nutrient Group = all phosphorus and nitrogen species that are commonly determined in lakes (total phosphorus, nitrate + nitrite, ammonia, total Kjeldahl nitrogen, total nitrogen)
} 


\section{Replicate Sampling}

R1. At all lakes in program, triplicate samples are taken near water surface in summer for analysis of total phosphorus and chlorophyll-a. At two of these lakes, a set of triplicate samples is also taken from near-bottom water, for analysis of total phosphorus.

R2. At three selected lakes in the spring (different lakes each year), triplicate samples are taken near water surface for analysis of Nutrient Group.

R3. At one lake each year, 5 replicate samples are taken near water surface for analysis of total phosphorus and chlorophyll-a.

\section{Standard Addition Testing}

S1. At Delavan Lake and one other lake (to be determined each year), 5 replicate samples are taken in August for a standard addition (spike) test. The spike consists of addition of a prepared phosphorus solution (standard) of known volume and concentration, such that the expected result of analysis is the natural water TP concentration plus the known addition. One sample from each set will receive a lowconcentration spike, one will receive a high-concentration spike, and three will receive no spike (the mean of these gives the natural water TP concentration).

Data and results of replicate sampling and field blank testing in water year 2000 are shown in Table A1. 
Table A1. Analyses of replicate samples from Wisconsin lakes in water years 2001-2004. See text for procedures used. All data except chlorophyll in milligrams per liter. Symbol "<" indicates less than given detection limit (DL); mean and standard deviation not calculated for datasets containing values less than DL. All samples collected near surface (approximately $0.5 \mathrm{~m}$ depth) unless otherwise indicated.

\begin{tabular}{|c|c|c|c|c|c|c|c|c|c|}
\hline Parameter & Lake & Date & \multicolumn{3}{|c|}{$\begin{array}{c}\text { Replicate } \\
\text { Data }\end{array}$} & & Mean & \multicolumn{2}{|c|}{$\begin{array}{r}\quad \text { Percent } \\
\text { ard Standard } \\
\text { tion Deviation } \\
\end{array}$} \\
\hline \multirow{23}{*}{$\begin{array}{c}\text { Total } \\
\text { Phosphorus }\end{array}$} & Buffalo & $7 / 23 / 01$ & 0.276 & 0.275 & 0.277 & & 0.276 & 0.001 & 0.4 \\
\hline & Delavan & $7 / 15 / 01$ & 0.027 & 0.027 & 0.031 & & 0.028 & 0.002 & 8.2 \\
\hline & Delavan & $8 / 19 / 01$ & 0.031 & 0.027 & 0.035 & & 0.031 & 0.004 & 12.9 \\
\hline & Geneva & $7 / 15 / 01$ & 0.005 & $<0.005$ & $<0.005$ & & & & \\
\hline & Little Green & $7 / 23 / 01$ & 0.069 & 0.074 & 0.072 & & 0.072 & 0.003 & 3.5 \\
\hline & Middle & $6 / 17 / 01$ & 0.012 & 0.012 & 0.017 & 0.016 & 0.014 & 0.003 & 18.5 \\
\hline & Muskego & $4 / 18 / 01$ & 0.039 & 0.044 & 0.047 & & 0.043 & 0.004 & 9.3 \\
\hline & Muskego & $7 / 25 / 01$ & 0.030 & 0.031 & 0.031 & & 0.031 & 0.001 & 1.9 \\
\hline & Oconomowoc & $7 / 17 / 01$ & 0.010 & 0.011 & 0.010 & & 0.010 & 0.001 & 5.6 \\
\hline & Oconomowoc & $8 / 23 / 01$ & 0.011 & 0.010 & 0.009 & & 0.010 & 0.001 & 10.0 \\
\hline & Okauchee & $8 / 20 / 01$ & 0.013 & 0.015 & 0.015 & & 0.014 & 0.001 & 8.1 \\
\hline & Red Cedar & $7 / 9 / 01$ & 0.021 & 0.022 & & & 0.022 & 0.001 & 3.3 \\
\hline & Delavan & $7 / 15 / 02$ & 0.026 & 0.026 & 0.027 & 0.031 & 0.028 & 0.002 & 8.7 \\
\hline & Geneva & $7 / 16 / 02$ & 0.008 & 0.008 & 0.008 & & 0.008 & 0.000 & 0.0 \\
\hline & Little Muskego & $7 / 1 / 02$ & 0.016 & 0.016 & 0.017 & & 0.016 & 0.001 & 3.5 \\
\hline & Potter & $8 / 5 / 02$ & 0.041 & 0.036 & 0.042 & $0.043 \quad 0.041$ & 0.041 & 0.003 & 6.7 \\
\hline & Little St. Germain & $7 / 22 / 02$ & 0.061 & 0.060 & 0.059 & & 0.060 & 0.001 & 1.7 \\
\hline & Delavan & $4 / 14 / 03$ & 0.057 & 0.057 & 0.057 & & 0.057 & 0.000 & 0.0 \\
\hline & Delavan & $8 / 12 / 03$ & 0.044 & 0.043 & 0.041 & & 0.043 & 0.002 & 3.6 \\
\hline & Lac La Belle & $8 / 19 / 03$ & 0.015 & 0.012 & 0.012 & & 0.013 & 0.002 & 13.3 \\
\hline & Butternut & $8 / 13 / 03$ & 0.040 & 0.042 & & & 0.041 & 0.001 & 3.5 \\
\hline & Delavan & $7 / 20 / 04$ & 0.031 & 0.020 & 0.041 & & 0.031 & 0.011 & $34.3^{*}$ \\
\hline & Big Cedar & $8 / 18 / 04$ & 0.012 & 0.011 & 0.012 & & 0.012 & 0.001 & 4.9 \\
\hline \multirow{4}{*}{$\begin{array}{c}\text { Total } \\
\text { Phosphorus, } \\
\text { near bottom }\end{array}$} & Geneva & $7 / 15 / 01$ & 0.017 & 0.020 & 0.021 & & 0.019 & 0.002 & 10.8 \\
\hline & Red Cedar & $7 / 9 / 01$ & 0.187 & 0.228 & 0.262 & & 0.226 & 0.038 & 16.6 \\
\hline & Wind & $7 / 8 / 02$ & 0.084 & 0.089 & 0.092 & & 0.088 & 0.004 & 4.6 \\
\hline & Wind & $8 / 19 / 03$ & 0.194 & 0.192 & 0.165 & & 0.184 & 0.016 & 8.8 \\
\hline \multirow{4}{*}{$\begin{array}{c}\text { Dissolved } \\
\text { Phosphorus }\end{array}$} & Delavan & $7 / 15 / 01$ & 0.010 & $<0.002$ & $<0.007$ & & & & \\
\hline & Geneva & $4 / 17 / 01$ & $<0.002$ & $<0.002$ & & & & & \\
\hline & Oconomowoc & $8 / 23 / 01$ & 0.002 & $<0.002$ & $<0.002$ & & & & \\
\hline & Delavan & $4 / 14 / 03$ & 0.022 & 0.023 & 0.023 & & 0.023 & 0.001 & 2.5 \\
\hline \multirow{5}{*}{$\begin{array}{l}\text { Dissolved } \\
\text { Ammonia }\end{array}$} & Delavan & $7 / 15 / 01$ & 0.026 & 0.013 & 0.021 & & 0.020 & 0.007 & 32.8 \\
\hline & Geneva & $4 / 17 / 01$ & 0.014 & 0.022 & & & 0.018 & 0.006 & 31.4 \\
\hline & Muskego & $4 / 18 / 01$ & 0.086 & 0.083 & 0.084 & & 0.084 & 0.002 & 1.8 \\
\hline & Oconomowoc & $8 / 23 / 01$ & 0.027 & 0.028 & 0.022 & & 0.026 & 0.003 & 12.5 \\
\hline & Delavan & $4 / 14 / 03$ & $<0.015$ & $<0.015$ & $<0.015$ & & & & \\
\hline
\end{tabular}

* Algal bloom on lake. 


\begin{tabular}{crrrrrrrr} 
& Delavan & $7 / 15 / 01$ & 0.560 & 0.580 & 0.560 & $\mathbf{0 . 5 6 7}$ & $\mathbf{0 . 0 1 2}$ & $\mathbf{2 . 0}$ \\
Total Kjeldahl & Geneva & $4 / 17 / 01$ & 0.390 & 0.390 & & $\mathbf{0 . 3 9 0}$ & $\mathbf{0 . 0 0 0}$ & $\mathbf{0 . 0}$ \\
Nitrogen & Muskego & $4 / 18 / 01$ & 1.200 & 1.100 & 1.200 & $\mathbf{1 . 1 6 7}$ & $\mathbf{0 . 0 5 8}$ & $\mathbf{4 . 9}$ \\
& Oconomowoc & $8 / 23 / 01$ & 0.490 & 0.500 & 0.520 & $\mathbf{0 . 5 0 3}$ & $\mathbf{0 . 0 1 5}$ & $\mathbf{3 . 0}$ \\
\cline { 2 - 8 } & Delavan & $4 / 14 / 03$ & 0.640 & 0.640 & 0.620 & $\mathbf{0 . 6 3 3}$ & $\mathbf{0 . 0 1 2}$ & $\mathbf{1 . 8}$ \\
\hline
\end{tabular}

Parameter

Lake Date

Replicate Data

Percent

Standard Standard

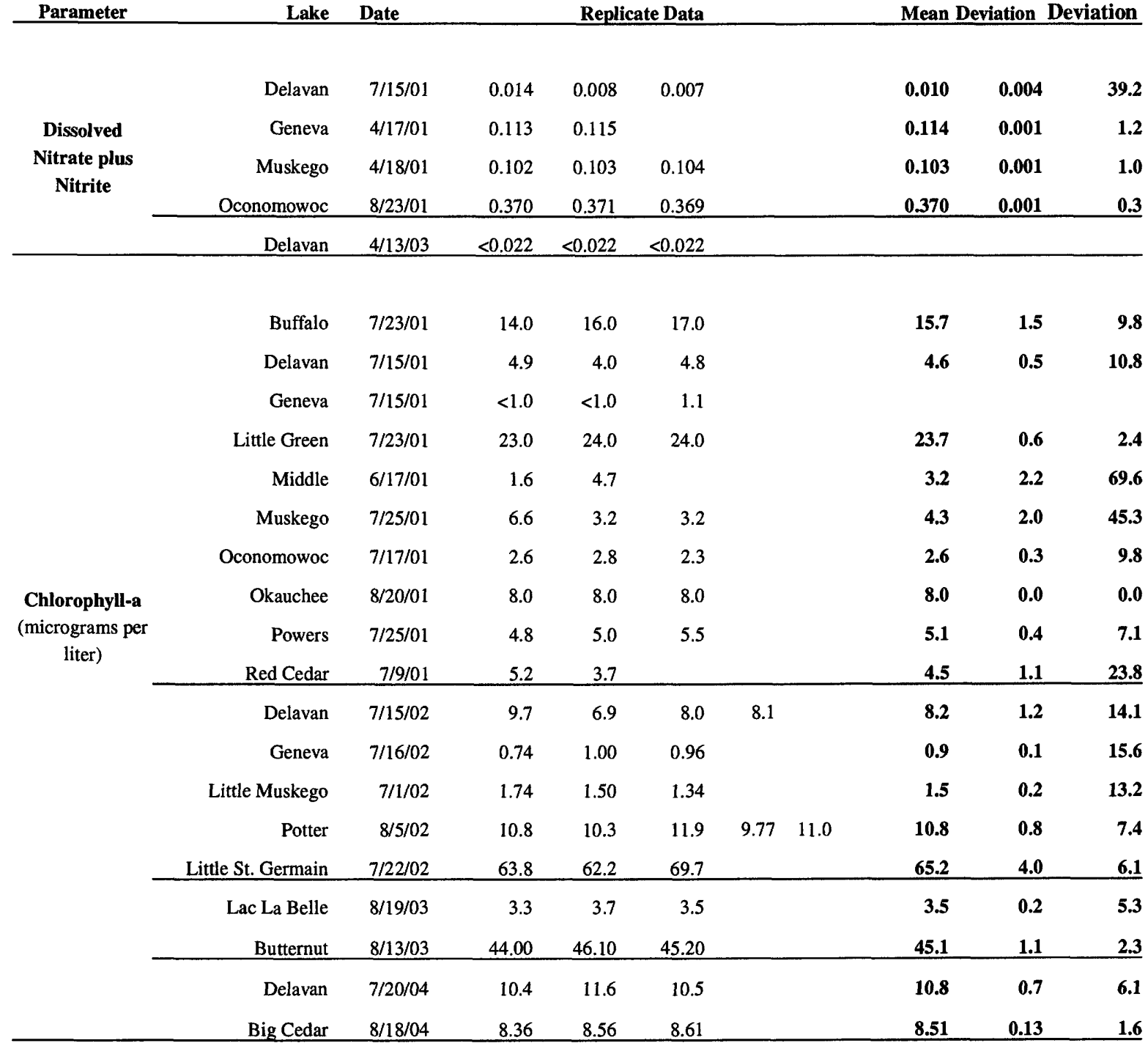

Table A2. Data from standard addition tests using stock solution containing $5.00 \mathrm{mg} / \mathrm{L}$ phosphorus. See text for detail of procedures. All concentration data in milligrams per liter.

\begin{tabular}{lccccc} 
Lake, Date & $\begin{array}{c}\text { Original } \\
\text { Sample } \\
\text { Concentration }\end{array}$ & $\begin{array}{c}\text { Stock Solution } \\
\text { Volume Added } \\
\text { (milliliters) }\end{array}$ & $\begin{array}{c}\text { Final Expected } \\
\text { Concentration }\end{array}$ & $\begin{array}{c}\text { Actual } \\
\text { Detected } \\
\text { Concentration }\end{array}$ & $\begin{array}{c}\text { Percent } \\
\text { Recovery }\end{array}$ \\
\hline Delavan 08/12/03 & 0.043 & 0.310 & 0.056 & 0.058 & $116 \%$ \\
& 0.043 & 1.250 & 0.094 & 0.099 & $108 \%$
\end{tabular}


Table A3. Data from tests of blanks, 2001-2004. All data in milligrams per liter, unless otherwise indicated.< = less than given detection limit; $\mathrm{E}=$ estimated value.

Delavan Lake. Analyses at USGS National Water Quality Laboratory, Lakewood, CO.

\begin{tabular}{|c|c|c|c|c|c|c|c|c|c|c|}
\hline Parameter & $2 / 19 / 01$ & $4 / 17 / 01$ & $7 / 15 / 01$ & $2 / 21 / 02$ & $4 / 17 / 02$ & \multicolumn{2}{|c|}{$7 / 14 / 02$} & $2 / 20 / 03$ & $4 / 16 / 03$ & $6 / 22 / 04$ \\
\hline Total P & E 0.003 & $<0.004$ & $<0.004$ & $<0.004$ & $<0.004$ & $<0.004$ & 0.006 & E 0.002 & $<0.004$ & $<0.004$ \\
\hline Dissolved orthophosphate & & & $<0.007$ & & & $<0.007$ & $<0.007$ & & $<0.007$ & \\
\hline Chlorophyll a & $<0.1$ & $<0.1$ & & $<0.1$ & & $<0.1$ & $<0.1$ & & $<0.260$ & $<0.260$ \\
\hline Chlorophyll b & $<0.1$ & & & $<0.1$ & & $<0.1$ & $<0.1$ & & & \\
\hline Total Kjeldahl Nitrogen (as N) & & & $<0.08$ & & $<0.10$ & E 0.05 & E 0.05 & & $<0.10$ & \\
\hline Nitrate + Nitrite (as N) & & & $<0.05$ & & & E 0.011 & 0.008 & & $<0.022$ & \\
\hline
\end{tabular}

Big Cedar Lake, south site, near West Bend, WI. Analyses at Wisconsin State Laboratory of Hygiene, Madison, WI

\begin{tabular}{lcc} 
Parameter & $4 / 22 / 02$ & $8 / 8 / 02$ \\
\hline Total P & $<0.005$ & $<0.005$ \\
Dissolved orthophosphate & $<0.002$ & \\
Chlorophyll a & $<1.00$ & $<0.26$ \\
Total Kjeldahl Nitrogen (as N) & $<0.14$ \\
Ammonia (as N) & $<0.013$ \\
Nitrate + Nitrite (as N) & $<0.010$ \\
Calcium, dissolved & $<0.20$ \\
Magnesium, dissolved & $<0.20$ \\
Potassium, dissolved & $<1.0$ \\
Sodium, dissolved & $<0.10$ \\
Iron, dissolved (micrograms per liter) & $<100$ \\
Manganese, dissolved (micrograms per liter) & $<1$ \\
& \\
\hline
\end{tabular}

Little Cedar Lake, south site, near West Bend, WI. Analyses at Wisconsin State Laboratory of Hygiene, Madison, WI

\begin{tabular}{lc} 
Parameter & $4 / 29 / 03$ \\
\hline Total P & $<0.005$ \\
Dissolved orthophosphate & $<0.002$ \\
Total Kjeldahl Nitrogen (as N) & $<0.14$ \\
Ammonia (as N) & $<0.013$ \\
Nitrate + Nitrite (as N) & 0.016
\end{tabular}

Little Cedar Lake, North site, near West Bend, WI. Analyses at Wisconsin State Laboratory of Hygiene, Madison, WI

\begin{tabular}{ll} 
Parameter & $2 / 21 / 03$ \\
\hline Total P & $<0.005$
\end{tabular}

